Supporting information for:

\title{
Molecular Modeling Based Approach to Potent P2-P4 Macrocyclic Inhibitors of Hepatitis C NS3/4A Protease
}

\author{
Nigel J. Liverton ${ }^{\ddagger}$, M. Katharine Holloway, John A. McCauley, Michael T. Rudd, John W. Butcher, \\ Steven S. Carroll, Jillian DiMuzio, Christine Fandozzi, Kevin F. Gilbert, Shi-Shan Mao, Charles J. \\ Mclntyre, Kevin T. Nguyen, Joseph J. Romano, Mark Stahlhut, Bang-Lin Wan David B. Olsen and \\ Joseph P. Vacca
}

\section{Contents}

Extended Reference Information

Molecular Modeling Information

General Experimental Information

Experimental Procedures

Compound Characterization data (NMR/HPLC)
S1

S2

S3

S4

S27

\section{Complete reference information:}

Ref 7a: Llinas-Brunet, M.; Bailey, M. D.; Bolger, G.; Brochu, C.; Faucher, A.-M.; Ferland, J. M.; Garneau, M.; Ghiro, E.; Gorys, V.; Grand-Maitre, C.; Halmos, T.; Lapeyre-Paquette, N.; Liard, F.; Poirier, M.; Rheaume, M.; Tsantrizos, Y. S.; Lamarre, D. J. Med. Chem., 2004, 47, 1605-1608.

Ref 7b: Lamarre, D.; Anderson, P. C.; Bailey, M.; Beaulieu, P.; Bolger, G.; Bonneau, P.; Boes, M.; Cameron, D. R.; Cartier, M.; Cordingley, M. G.; Faucher, A.-M.; Goudreau, N.; Kawai, S. H.; Kukolj, G.; Lagace, L.; LaPlante, S. R.; Narjes, H.; Poupart, M.-A.; Rancourt, J.; Sentjens, R. E.; St. George, R.; Simoneau, B.; Steinmann, G.; Thibeault, D.; Tsantrizos, Y. S.; Weldon, S. M.; Yong, C.-L.; LlinasBrunet, M. Nature, 2003, 426, 186-189.

Ref 8a: Perni, R. B.; Almquist, S. J.; Byrn, R. A.; Chandorkar, G.; Chaturvedi, P. R.; Courtney, L. F.; Decker, C. J.; Dinehart, K.; Gates, C. A.; Harbeson, S. L.; Heiser, A.; Kalkeri, G.; Kolaczkowski, E.; Lin, K.; Luong, Y.-P.; Rao, B. G.; Taylor, W. P.; Thomson, J. A.; Tung, R. D.; Wei, Y.; Kwong, A. D.; Lin, C. Antimicrob. Agents Chemother., 2006, 50, 899-909.

Ref 14: Migliaccio, G.; Tomassini, J. E.; Carroll, S. S.; Tomei, L.; Altamura, S.; Bhat, B.; Bartholomew, L.; Bosserman, M. R.; Ceccacci, A.; Colwell, L. F.; Cortese, R.; De Francesco, R.; Eldrup, A. B.; Getty, K. L.; Hou, X. S.; LaFemina, R. L.; Ludmerer, S. W.; MacCoss, M.; McMasters, D. R.; Stahlhut, M. W.; Olsen, D. B.; Hazuda, D.J.; Flores, O. A. J. Biol. Chem. 2003, 278, 49164-49170. 


\section{Molecular Modeling Methods:}

A model of inhibitor 1 was created in the NS3/NS4A active site (monomer A of the 1CU1 crystal structure $^{1}$ ) based on the binding mode previously described. ${ }^{2}$ Models of 3a-3d were subsequently derived from the model of $\mathbf{1}$. The flexibility of the macrocyclic linker in 3a-3d was explored by generating 25 conformers using a distance geometry algorithm. ${ }^{3}$ Bound poses were energy-minimized using the MMFFs force field ${ }^{4}$ with a distance-dependent dielectric constant $(\varepsilon=2 r)$ in the rigid NS3/NS4A active site defined by selecting all residues within $10 \AA$ of any atom of the model of $\mathbf{1}$. All titratable enzyme residues were charged and all inhibitors were defined as carboxylates. The pose which resulted in the lowest conformational energy for the ligand was selected and the corresponding enzymeligand energy $\left(\mathrm{E}_{\mathrm{inter}}\right)$ was compared to the observed $\mathrm{pK}$. The energy-minimized geometries were also subsequently rescored using $\mathrm{X}-\mathrm{Score}^{5}$ in the same active site.

1. Molecular views of viral polyprotein processing revealed by the crystal structure of the hepatitis C virus bifunctional protease-helicase. Yao, N.; Reichert, P.; Taremi, S. S.; Prosise, W. W.; Weber, P. C. Structure 1999, 7, 1353-1363.

2. Macrocyclic Inhibitors of the NS3 Protease as Potential Therapeutic Agents of Hepatitis C Virus Infection. Tsantrizos, Y. S.; Bolger, G.; Bonneau, P.; Cameron, D. R.; Goudreau, N.; Kukolj, G.; LaPlante, S. R.; Llinàs-Brunet, M.; Nar, H.; Lamarre, D. Angewandte Chemie International Edition 2003, 42, 1356-1360.

3. In-house distance geometry program written using the theory and algorithms from: Crippen, C. M.; Havel, T. F., D. Bawden, Ed.; Research Studies Press, Wiley, New York, 1988 and Kuszewski, J.; Nilges, M.; Brunger, A. T. J. of Biomol. NMR, 1992, 2, 33.

4. (a) Halgren, T. A. J. Comput. Chem. 1996, 17, 490, 520, 553, and 616; (b) Halgren, T. A.; Nachbar, R. B. J. Comput. Chem. 1996, 17, 587.

5. Wang, R.; Lu, Y.; Fang, X.; Wang, S. J. Chem. Inf. Comput. Sci. 2004, 44, 2114. 


\section{General Experimental Information}

All reagents and solvents were of commercial quality and used without further purification unless indicated otherwise. All reactions were carried out under an inert atmosphere of nitrogen.

${ }^{1} \mathrm{H}$ NMR spectra were obtained on a Varian VXR-300S spectrometer, a Varian Unity Inova 400 spectrometer or a Varian Unity Inova 500 spectrometer. Chemical shifts are reported in parts per million relative to TMS as internal standard.

Samples provided for accurate mass measurement were taken up in acetonitrile:water:glacial acetic acid $(50: 50: 0.1 \% \mathrm{v} / \mathrm{v})$. The solutions were analyzed by use of electrospray ionization (ESI) or atmospheric pressure chemical ionization (APCI) on either a Bruker Daltonics 3T or 7T Fourier transform ion cyclotron resonance (FTICR) mass spectrometer. External calibration was accomplished with polypropylene glycol (425 or 750$)$.

Melting points were determined in open glass capillaries using a Thomas-Hoover UniMelt melting point apparatus and are uncorrected.

Elemental analyses were performed by Quantitative Technologies Inc., Whitehouse, NJ.

Silica gel chromatography was carried out with an ISCO CombiFlash Sg 100c or CombiFlash Companion purification system using ISCO RediSep silica gel cartridges.

Preparative reverse-phase HPLC was performed using a Gilson 215 liquid handler and a Phenomenex Luna C18 column (150 x 20 mm I.D.) with a linear gradient over 15 minutes (95:5 to 5:95 H2O:acetonitrile, both containing 0.1\% trifluoroacetic acid) with UV detection at $220 \mathrm{~nm}$.

Analytical HPLC data was obtained by two methods on an Agilent 1090 HPLC with binary pump and diode array detector with area quantitation performed at $214 \mathrm{~nm}$.

Method 1: Zorbax RX-C18, 75 x 4.6 mm, $3.5 \mu \mathrm{M}, 98 \%$ A 2\% B to 100\% B over 5.5 min then 100\% B to $6.0 \mathrm{~min}(\mathrm{~A}=0.1 \% \mathrm{H3PO} 4 /$ water $\mathrm{v} / \mathrm{v} ; \mathrm{B}=$ acetonitrile $)$.

Method 2: Luna C8(2), $3 \mu \mathrm{M}, 75$ x $4.6 \mathrm{~mm}, 98 \%$ A $2 \%$ B to $100 \%$ B over 5.5 min then $100 \%$ B to 6.0 $\min (\mathrm{A}=0.1 \% \mathrm{H3PO} 4 /$ water $\mathrm{v} / \mathrm{v} ; \mathrm{B}=$ acetonitrile $)$. 


\section{Experimental Procedures}

\section{1-t-Butyl 2-methyl (2S,4S)-4-\{[(4-bromophenyl)sulfonyl]oxy\}pyrrolidine-1,2-dicarboxylate (4)}<smiles>CC(=O)OC1CC(OS(=O)(=O)c2ccc(Br)cc2)CN1C(=O)c1ccccc1</smiles>

4 was prepared as described previously (Arasappan, A.; Chen, K. X.; Njoroge, F. G.; Parekh, T. N.; Girijavallabhan, V. J. Org. Chem., 2002, 67, 3923-3926).

\section{6-Bromo-2-phenylquinolin-4-ol (5)}

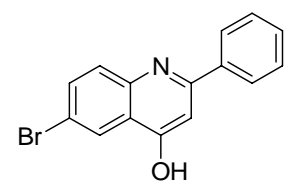

5 was prepared as described previously (Kaslow, C. E.; Lawton, W.R. J. Org. Chem., 1950, 72, 17231724).

1-t-Butyl 2-methyl (2S,4R)-4-[(6-bromo-2-phenylquinolin-4-yl)oxy]pyrrolidine-1,2-dicarboxylate (6)

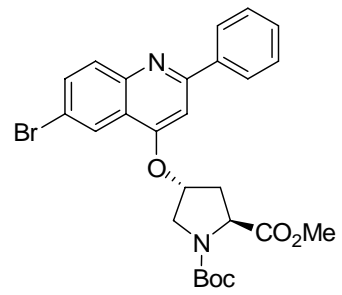

To a stirred solution of 5 ( $776 \mathrm{mg}, 2.58 \mathrm{mmol})$ and 4 (1.0 g, $2.15 \mathrm{mmol})$ in N-methylpyrrolidinone (10 $\mathrm{mL}$ ) was added $\mathrm{Cs}_{2} \mathrm{CO}_{3}(1.05 \mathrm{~g}, 3.23 \mathrm{mmol})$. The reaction mixture was stirred at $40{ }^{\circ} \mathrm{C}$ for $18 \mathrm{~h}$, cooled to RT and partitioned between water and EtOAc $(2 \times 100 \mathrm{~mL})$. The combined organic phases were washed with brine, dried over anhydrous $\mathrm{Na}_{2} \mathrm{SO}_{4}$ and solvent evaporated in vacuo. The crude product was purified on silica gel, eluting with 10-50\% EtOAc/hexane, to afford 6 as a white solid (978 mg, 86\%). ${ }^{1} \mathrm{H}$ NMR (400 MHz, $\mathrm{CDCl}_{3}$ ) (mixture of Boc rotamers) $\delta 8.25$ (brs, $1 \mathrm{H}$ ), 8.05 (brt, $J=8.2 \mathrm{~Hz}, 2$ H), 7.96 (d, $J=9.0 \mathrm{~Hz}, 1 \mathrm{H}$ ), 7.77 (dd, $J=9.0,2.1 \mathrm{~Hz}, 1 \mathrm{H}$ ), 7.44-7.56 (m, $3 \mathrm{H}$ ), 7.08 (brs, $1 \mathrm{H}$ ), 5.22$5.34(\mathrm{~m}, 1 \mathrm{H}), 4.64(\mathrm{t}, J=7.5 \mathrm{~Hz}, 1 \mathrm{H}$ from minor diastereomer), 4.56 (t, $J=7.9 \mathrm{~Hz}, 1 \mathrm{H}$ from major diastereomer) 3.75-4.05 (m, $5 \mathrm{H}), 2.66-2.82$ (m, $1 \mathrm{H}), 2.40-2.49$ (m, $1 \mathrm{H}), 1.45$ (s, $9 \mathrm{H})$ ppm; HRMS (ESI) $m / z 527.1188\left[(\mathrm{M}+\mathrm{H})^{+}\right.$; calcd for $\left.\mathrm{C}_{26} \mathrm{H}_{28} \mathrm{BrN}_{2} \mathrm{O}_{5}: 527.1176\right]$. 


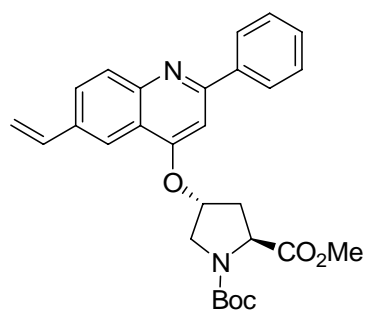

A solution of 6 (921 mg, $1.75 \mathrm{mmol})$ in toluene (20 mL) was degassed by bubbling nitrogen through for 15 min. Vinyltributyltin (769 $\mu \mathrm{L}, 2.62 \mathrm{mmol}$ ) and tetrakis(triphenylphosphine)palladium (0) (202 mg, $0.175 \mathrm{mmol}$ ) were added and the mixture heated to $100{ }^{\circ} \mathrm{C}$ for $4 \mathrm{~h}$. The dark reaction mixture was concentrated in vacuo and purified on silica gel, eluting with 10-50\% EtOAc/hexane, to afford 7 as a white solid (653 mg, 79\%). ${ }^{1} \mathrm{H}$ NMR (400 MHz, $\mathrm{CDCl}_{3}$ )(mixture of Boc rotamers) $\delta$ 8.0-8.1 (m, $4 \mathrm{H}$ ), 7.88, dd, $J=8.9,2.0 \mathrm{~Hz}, 1 \mathrm{H}), 7.44-7.53$ (m, $3 \mathrm{H}), 7.04-7.08$ (m, $1 \mathrm{H}), 6.86-6.96(\mathrm{~m}, 1 \mathrm{H}), 5.90(\mathrm{~d}, J=$ $17.6 \mathrm{~Hz}, 1 \mathrm{H}), 5.39 \mathrm{~d}, J=11.0 \mathrm{~Hz}, 1 \mathrm{H}), 5.24-5.34(\mathrm{~m}, 1 \mathrm{H}), 4.65$ (t, $J=7.6 \mathrm{~Hz}, 1 \mathrm{H}$ of minor rotamer), 4.55 (t, $J=7.9 \mathrm{~Hz}, 1 \mathrm{H}$ of major rotamer) 3.8-4.1 (m, $5 \mathrm{H}), 2.72-2.84$ (m, $1 \mathrm{H}), 2.40-2.49$ (m, $1 \mathrm{H}), 1.45$ (s, 9H) ppm; HRMS (ESI) m/z 475.2231 [(M+H) ${ }^{+}$; calcd for $\mathrm{C}_{28} \mathrm{H}_{31} \mathrm{~N}_{2} \mathrm{O}_{5}$ : 475.2228].

\section{N-[(allyloxy)carbonyl]-L-norleucine (8a)}

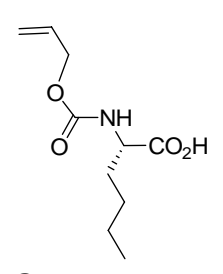

8a was prepared as described previously (Debaene, F.; Mejias, L.; Harris, J. L.; Winssinger, N. Tetrahedron, 2004, 60, 8677-8690).

\section{N-[(But-3-en-1-yloxy)carbonyl]-L-norleucine (8b)}

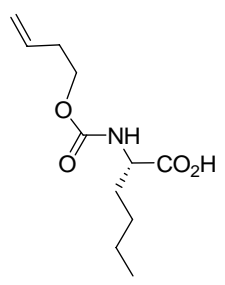

To a solution of 3-buten-1-ol (794 mg, $11 \mathrm{mmol})$ in DMF (15 mL) at $0{ }^{\circ} \mathrm{C}$ was added CDI (1.785 g, 11 mmol). The reaction mixture was allowed to warm to RT, stirred for $30 \mathrm{~min}$ and a solution of Lnorleucine methyl ester hydrochloride $(2.0 \mathrm{~g}, 11 \mathrm{mmol})$ in DMF (10 mL) added. The reaction mixture was warmed to $50{ }^{\circ} \mathrm{C}$ for $3 \mathrm{~h}$, cooled to RT, stirred for $60 \mathrm{~h}$ and partitioned between Et2O and water. The organic phase was washed with brine, dried over anhydrous $\mathrm{Na}_{2} \mathrm{SO}_{4}$ and solvent evaporated in vacuo. The crude product was purified on silica gel, eluting with 10-90\% EtOAc/hexane, to afford a foam which was dissolved in THF (25 mL) and $\mathrm{NaOH}(25 \mathrm{~mL}, 1 \mathrm{M})$ added. The reaction mixture was stirred at RT for $3 \mathrm{~h}$, then partitioned between EtOAc and $1 \mathrm{M} \mathrm{HCl} \mathrm{(x} \mathrm{2)} \mathrm{and} \mathrm{the} \mathrm{organic} \mathrm{phase} \mathrm{washed}$ with water and brine, dried over Na2SO4 and solvent evaporated in vacuo to afford product as a clear oil 1.30g 77\%). ${ }^{1} \mathrm{H}$ NMR (400 MHz, $\left.\mathrm{CD}_{3} \mathrm{OD}\right) \delta$ 5.77-5.88 (m, $1 \mathrm{H}$ ), 5.11 (dd, $J=17.2,1.6 \mathrm{~Hz}, 1 \mathrm{H}$ ), 5.03- 
5.07 (m, 1 H), 4.04-4.12 (m, 3 H), 2.37 (q, $J=6.7$ Hz, 2 H), 1.75-1.85 (m, $1 \mathrm{H}), 1.59-1.70$ (m, $1 \mathrm{H}$ ), 1.30-1.42 (m, $4 \mathrm{H}), 0.92$ (t, $J=7.1 \mathrm{~Hz}, 3 \mathrm{H}) \mathrm{ppm}$; HRMS (ESI) $\mathrm{m} / \mathrm{z} 230.1388\left[(\mathrm{M}+\mathrm{H})^{+}\right.$; calcd for $\mathrm{C}_{11} \mathrm{H}_{20} \mathrm{NO}_{4}$ : 231.1387].

\section{N-[(Pent-4-en-1-yloxy)carbonyl]-L-norleucine (8c)}

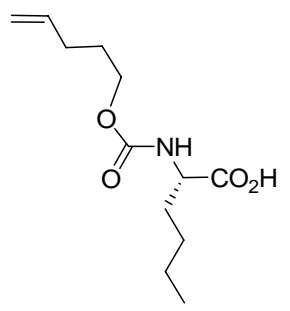

Prepared in the same manner as $\mathbf{8 b}(74 \%) .{ }^{1} \mathrm{H}$ NMR (400 MHz, $\left.\mathrm{CD}_{3} \mathrm{OD}\right) \delta 5.76-5.88$ (m, $\left.1 \mathrm{H}\right), 5.04$ (dq, $J=17.1,1.7 \mathrm{~Hz}, 1 \mathrm{H}), 4.95-4.99$ (m, $1 \mathrm{H}), 4.10$ (dd, $J=9.1,4.9 \mathrm{~Hz}, 1 \mathrm{H}), 4.05$ (t, $J=6.6 \mathrm{~Hz}, 2 \mathrm{H}), 2.14$ (q, $J=7.2 \mathrm{~Hz}, 2 \mathrm{H}), 1.76-1.86$ (m, $1 \mathrm{H}), 1.60-1.74$ (m, $3 \mathrm{H}), 1.30-1.42$ (m, $4 \mathrm{H}), 0.92$ (t, $J=7.0 \mathrm{~Hz}, 3 \mathrm{H}$ ) ppm; HRMS (ESI) $\mathrm{m} / \mathrm{z} 244.1594\left[(\mathrm{M}+\mathrm{H})^{+}\right.$; calcd for $\mathrm{C}_{12} \mathrm{H}_{22} \mathrm{NO}_{4}$ : 244.1544].

\section{$\mathrm{N}$-[(Hex-5-en-1-yloxy)carbonyl]-L-norleucine (8d)}

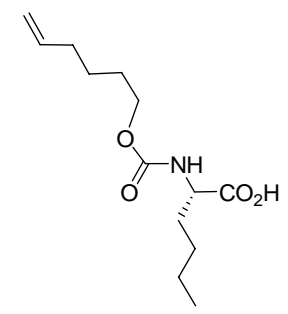

Prepared in the same manner as $\mathbf{8 b}(47 \%) .{ }^{1} \mathrm{H}$ NMR (400 MHz, $\left.\mathrm{CD}_{3} \mathrm{OD}\right) \delta 5.75-5.86$ (m, $1 \mathrm{H}$ ), 5.01 (dq, $J=17.1,1.7 \mathrm{~Hz}, 1 \mathrm{H}), 4.94$ (m, $1 \mathrm{H}), 4.05-4.14$ (m, $1 \mathrm{H}), 4.04$ (d, $J=6.5 \mathrm{~Hz}, 2 \mathrm{H}), 2.04-2.12$ (m, $2 \mathrm{H})$, 1.76-1.86 (m, 1 H), 1.59-1.70 (m, 3 H), 1.42-1.52 (m, 2 H), 1.30-1.40 (m, 4 H), 0.92 (t, $J=7.0$ Hz, 3 H) ppm; HRMS (ESI) $\mathrm{m} / \mathrm{z} 258.1705\left[(\mathrm{M}+\mathrm{H})^{+}\right.$; calcd for $\mathrm{C}_{13} \mathrm{H}_{24} \mathrm{NO}_{4}$ : 258.1700].

\section{Methyl N-[(allyloxy)carbonyl]-L-norleucyl-(4R)-4-[(2-phenyl-6-vinylquinolin-4-yl)oxy]-L-} prolinate (9a)

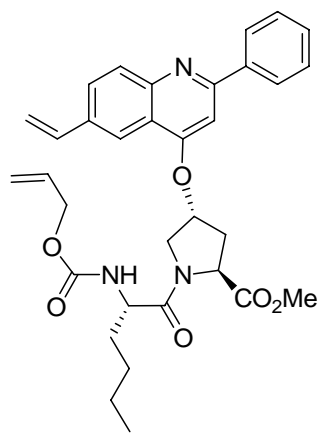

To 7 (608 $\mathrm{mg}, 1.28 \mathrm{mmol}$ ) was added $\mathrm{HCl}$ in dioxane $(15 \mathrm{~mL}, 4 \mathrm{M})$ and the mixture stirred for $2 \mathrm{~h}$. The volatiles were evaporated in vacuo and the residual solid azeotroped with EtOAc (x3) and suspended in DMF (10 mL). N-[(allyloxy)carbonyl]-L-norleucine 8a (303 mg, $1.41 \mathrm{mmol}$ ) was added, followed by 
DIEA (671 $\mu \mathrm{L}, 3.84 \mathrm{mmol})$, DMAP (78 mg, $0.64 \mathrm{mmol}$ ) and HATU (633 mg, $1.67 \mathrm{mmol}$ ). The reaction mixture was stirred at RT for $18 \mathrm{~h}$, partitioned between pH5.2 citrate buffer and EtOAc (x2). The combined organic phases were washed with water and brine, dried over anhydrous $\mathrm{Na}_{2} \mathrm{SO}_{4}$ and solvent evaporated in vacuo. The crude product was purified on silica gel, eluting with $10-60 \%$ EtOAc/hexane, to afford 9a as a foam (586 mg, 80\%). ${ }^{1} \mathrm{H}$ NMR (400 $\mathrm{MHz} \mathrm{CDCl}_{3}$ ) (mixture of Boc rotamers, major rotamer) $\delta$ 7.84-8.10 (m, 5 H), 7.44-7.56 (m, 3 H), 7.05 (s, 1 H), 6.91 (dd, J = 17.6, 10.9 Hz, 1 H), 5.89 (d, $J=17.6 \mathrm{~Hz}, 1 \mathrm{H}), 5.79-5.90(\mathrm{~m}, 1 \mathrm{H}), 5.1-5.45(\mathrm{~m}, 5 \mathrm{H}), 4.81(\mathrm{t}, J=17.6 \mathrm{~Hz}, 1 \mathrm{H}), 4.3-4.55$ (m, 4 H), 4.07 (dd, $J=4.2,11.4$ Hz, 1 H), 3.78 (s, 3 H), 2.75-2.83 (m, 1 H), 2.37-2.46 (m, $1 \mathrm{H}), 1.58-1.90$ (m, $2 \mathrm{H}), 1.25-1.44$ (m, $4 \mathrm{H}), 0.93$ (t, $J=7 \mathrm{~Hz}, 1 \mathrm{H}$ ) ppm; HRMS (ESI) m/z 572.2765 [(M+H) ${ }^{+}$; calcd for $\mathrm{C}_{33} \mathrm{H}_{38} \mathrm{~N}_{3} \mathrm{O}_{6}:$ 572.2755].

Methyl $N$-[(but-3-en-1-yloxy)carbonyl]-L-norleucyl-(4R)-4-[(2-phenyl-6-vinylquinolin-4-yl)oxy]-Lprolinate $(9 b)$

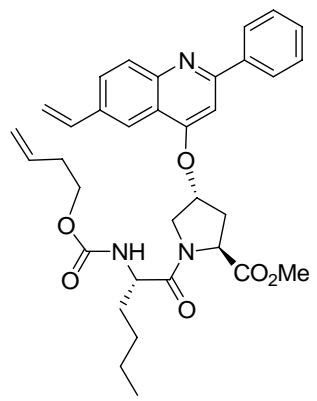

Prepared in the same manner as 9a (52\%). ${ }^{1} \mathrm{H} \mathrm{NMR}\left(400 \mathrm{MHz}, \mathrm{CDCl}_{3}\right.$ ) (mixture of Boc rotamers, major rotamer) $\delta$ 8.04-8.09 (m, 3 H), 7.97 (brs, 1 H), 7.88 (dd, $J=8.8,1.9$ Hz, 1 H), 7.45-7.56 (m, 3 H), 7.06 (s, $1 \mathrm{H}), 6.91$ (dd, $J=17.6,10.9 \mathrm{~Hz}, 1 \mathrm{H}), 5.89$ (d, $J=17.6 \mathrm{~Hz}, 1 \mathrm{H}), 5.64-5.78$ (m, $1 \mathrm{H})$, 5.32-5.42 (m, $3 \mathrm{H})$, 4.98-5.03 (m, $1 \mathrm{H}), 4.81$ (t, $J=8.2 \mathrm{~Hz}, 1 \mathrm{H}), 4.45-4.53$ (m, $1 \mathrm{H}), 4.36$ (brd, $J=11.5 \mathrm{~Hz}, 1 \mathrm{H}$ ), 3.88-4.12 (m, $3 \mathrm{H}), 3.79$ (s, $3 \mathrm{H}), 2.76-2.84$ (m, $1 \mathrm{H}), 2.38-2.46$ (m, $1 \mathrm{H}), 2.28$ (q, J = 6.7 Hz, $1 \mathrm{H})$, 1.761.88 (m, $1 \mathrm{H}), 1.58-1.70$ (m, $1 \mathrm{H}), 1.20-1.45$ (m, $4 \mathrm{H}), 0.8-0.94$ (m, $4 \mathrm{H}$ ) ppm; HRMS (ESI) m/z 586.2904 [(M+H) ${ }^{+}$; calcd for $\mathrm{C}_{34} \mathrm{H}_{40} \mathrm{~N}_{3} \mathrm{O}_{6}$ : 586.2912].

Methyl N-[(pent-4-en-1-yloxy)carbonyl]-L-norleucyl-(4R)-4-[(2-phenyl-6-vinylquinolin-4-yl)oxy]L-prolinate (9c)

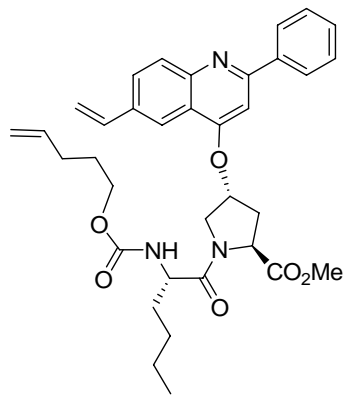

Prepared in the same manner as 9a (52\%). ${ }^{1} \mathrm{H}$ NMR $\left(400 \mathrm{MHz}, \mathrm{CDCl}_{3}\right.$ ) (mixture of Boc rotamers, major rotamer) $\delta 8.30$ (d, $J=8.8 \mathrm{~Hz}, 1 \mathrm{H}), 8.00-8.08$ (m, $2 \mathrm{H}), 7.86-7.93(\mathrm{~m}, 2 \mathrm{H}), 6.95$ (dd, $J=17.5,10.9 \mathrm{~Hz}$, $1 \mathrm{H}), 5.97$ (d, $J=17.6 \mathrm{~Hz}, 1 \mathrm{H}), 5.65-5.85$ (m, $2 \mathrm{H}), 5.55$ (d, $J=11 \mathrm{~Hz}, 1 \mathrm{H}), 5.24$ (d, $J=8.4 \mathrm{~Hz}, 1 \mathrm{H}$ ), 4.94-5.05 (m, 2 H), 4.82 (t, $J=8.4$ Hz, 1 H), 4.50 (d, $J=12.1 \mathrm{~Hz}, 1 \mathrm{H}), 4.41$ (q, J = 7.1 Hz, $1 \mathrm{H}$ ), 4.05- 
4.12 (m, $1 \mathrm{H}$ ), 3.70-3.80 (m, $1 \mathrm{H}), 3.79$ (s, $3 \mathrm{H}$ ), 3.70-3.77 (m, $1 \mathrm{H}), 2.75-2.85$ (m, $1 \mathrm{H}), 2.45-2.55$ (m, 1 H), 1.95-2.10 (m, 2 H), 1.50-1.90 (m, 4 H), 1.30-1.45 (m, 4 H), 0.92 (t, $J=7.0$ Hz, 3 H) ppm; HRMS (ESI) $\mathrm{m} / \mathrm{z} 600.3058\left[(\mathrm{M}+\mathrm{H})^{+}\right.$; calcd for $\left.\mathrm{C}_{35} \mathrm{H}_{42} \mathrm{~N}_{3} \mathrm{O}_{6}: 600.3068\right]$.

Methyl N-[(hex-5-en-1-yloxy)carbonyl]-L-norleucyl-(4R)-4-[(2-phenyl-6-vinylquinolin-4-yl)oxy]-Lprolinate (9d)

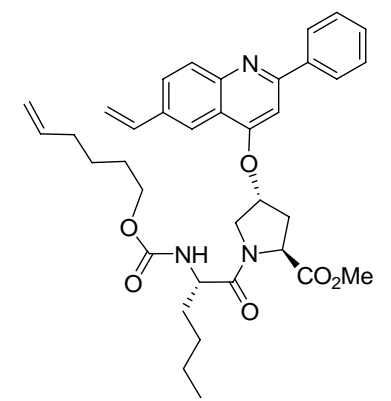

Prepared in the same manner as 9a (64\%). ${ }^{1} \mathrm{H}$ NMR $\left(400 \mathrm{MHz}, \mathrm{CDCl}_{3}\right)$ (mixture of Boc rotamers, major rotamer) $\delta$ 8.02-8.09 (m, $3 \mathrm{H}$ ), 7.97 (brs, $1 \mathrm{H}$ ), 7.88 (dd, $J=8.8,1.9 \mathrm{~Hz}, 1 \mathrm{H}$ ), 7.45-7.56 (m, $3 \mathrm{H}$ ), 7.06 (s, 1 H), 6.91 (dd, $J=17.6,10.9 \mathrm{~Hz}, 1 \mathrm{H}$ ), 5.89 (d, $J=17.6 \mathrm{~Hz}, 1 \mathrm{H}), 5.70-5.82$ (m, $1 \mathrm{H}$ ), 5.28-5.40 (m, 3 H), 4.90-5.05 (m, 1 H), 4.81 (, $J=8.2$ Hz, 1 H), 4.45-4.53 (m, 1 H), 4.36 (d, $J=11.6$ Hz, 1 H), 4.08 (dd, $J=11.3,4.3 \mathrm{~Hz}, 1 \mathrm{H}$ ), 3.93-4.00 (m, $1 \mathrm{H}$ ), 3.83-3.90 (m, $1 \mathrm{H}$ ), 3.78 (s, $3 \mathrm{H}$ ), 2.76-2.84 (m, $1 \mathrm{H}$ ), 2.37-2.46 (m, $1 \mathrm{H}), 2.02$ (q, $J=7.2 \mathrm{~Hz}, 2 \mathrm{H}), 1.30-1.90(\mathrm{~m}, 11 \mathrm{H}), 0.93(\mathrm{t}, J=7.0 \mathrm{~Hz}, 3 \mathrm{H}) \mathrm{ppm}$; HRMS (ESI) $\mathrm{m} / \mathrm{z} 614.3196\left[(\mathrm{M}+\mathrm{H})^{+}\right.$; calcd for $\mathrm{C}_{36} \mathrm{H}_{44} \mathrm{~N}_{3} \mathrm{O}_{6}$ : 614.3225].

Methyl (3R,5S,8S,13Z)-8-butyl-7,10-dioxo-20-phenyl-2,11-dioxa-6,9,19triazatetracyclo[13.6.2.1 3,6 .0 18,22 ]tetracosa-1(21),13,15,17,19,22-hexaene-5-carboxylate (10a)

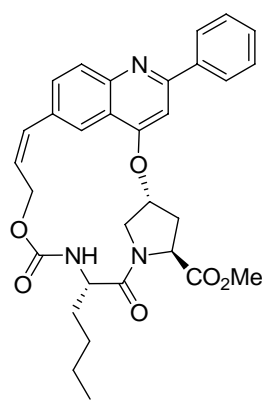

Nitrogen was bubbled through a solution of 9a (261 mg, $0.46 \mathrm{mmol})$ in 1,2-dichloroethane (100 mL) for 15 min, before addition of 1,3-bis(2,4,6-trimethylphenyl)-4,5-dihydroimidazol-2-ylidene[2-(i-propoxy)5-(N,N-dimethylaminosulfonyl)phenyl]methyleneruthenium (II) dichloride (Strem catalog \# 44-0082). The reaction mixture was stirred at room temperature under nitrogen for $24 \mathrm{~h}$, concentrated to approximately $5 \mathrm{~mL}$ and applied to a silica column, eluting with 10-100\% EtOAc hexane to afford product as a solid (210 mg, 85\%). ${ }^{1} \mathrm{H}$ NMR (400 MHz, $\left.\mathrm{CDCl}_{3}\right) \delta 8.38$ (d, $\left.J=8.8 \mathrm{~Hz}, 1 \mathrm{H}\right), 7.92-7.99$ (m, $3 \mathrm{H}), 7.74$ (dd, $J=8.9$, $1.8 \mathrm{~Hz}, 1 \mathrm{H}), 7.39-7.46$ (m, $3 \mathrm{H}), 7.19$ (s, $1 \mathrm{H}), 6.90$ (d, $J=11.4 \mathrm{~Hz}, 1 \mathrm{H})$, 6.21 (dt, $J=6.5$, 11.2 Hz, 1 H), 5.54-5.60 (m, 1 H), 5.41 (d, $J=10.4$ Hz, 1 H), 5.32 (d, $J=12.5$ Hz, 1 H), $5.27(\mathrm{t}, J=11.2 \mathrm{~Hz}, 1 \mathrm{H}), 4.76(\mathrm{t}, J=8.7 \mathrm{~Hz}, 1 \mathrm{H}), 4.62-4.72(\mathrm{~m}, 1 \mathrm{H}), 4.31$ (dd, $J=11.0,6.5 \mathrm{~Hz}, 1$ H), 3.99 (dd, $J=12.4,2.9$ Hz, 1 H), 3.77 (s, 3 H), 2.72-2.79 (m, 1 H), 2.44-2.56 (m, 1 H), 1.86-1.98 (m, 
$1 \mathrm{H}), 1.72-1.82$ (m, 1 H), 1.36-1.46 (m, 4 H), 0.92-1.00 (m, 3 H) ppm; HRMS (ESI) m/z 544.2448 $\left[(\mathrm{M}+\mathrm{H})^{+}\right.$; calcd for $\left.\mathrm{C}_{31} \mathrm{H}_{34} \mathrm{~N}_{3} \mathrm{O}_{6}: 544.2442\right]$.

Methyl (3R,5S,8S,14E)-8-butyl-7,10-dioxo-21-phenyl-2,11-dioxa-6,9,20triazatetracyclo[14.6.2.1 3,6 .0 19,23 ]pentacosa-1(22),14,16,18,20,23-hexaene-5-carboxylate (10b)

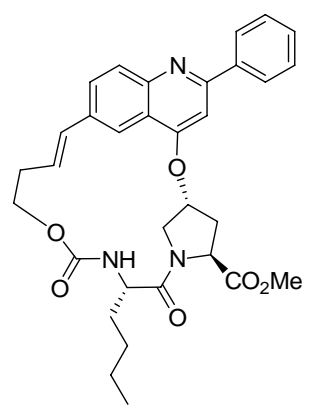

Prepared in the same manner as 10a (93\%). ${ }^{1} \mathrm{H}$ NMR (400 MHz, $\mathrm{CDCl}_{3}$ ) (mixture of Boc rotamers, major rotamer) $\delta 8.25$ (d, $J=8.8 \mathrm{~Hz}, 1 \mathrm{H}), 7.99(\mathrm{~d}, J=1.5 \mathrm{~Hz}, 1 \mathrm{H}), 7.88(\mathrm{~d}, J=6.8 \mathrm{~Hz}, 2 \mathrm{H}), 7.73$ (dd, $J=8.8,1$. Hz, 1 H), 7.30-7.40 (m, 3 H), 7.15 (s, 1 H), 6.66 (d, $J=15.9$ Hz, 1 H), 6.18-6.28 (m, 1 H), 5.80 (brs, $1 \mathrm{H}$ ), 5.53 (d, $J=9.4 \mathrm{~Hz}, 1 \mathrm{H}), 4.79-4.90$ (m, $2 \mathrm{H}), 4.76(\mathrm{t}, J=8.7 \mathrm{~Hz}, 1 \mathrm{H}), 4.66$ (d, $J=11.1$ Hz, 1 H), 3.94-4.06 (m, 2 H), 3.75 (s, 3 H), 2.68-2.80 (m, 2 H), 2.36-2.46 (m, 2 H), 1.70-1.90 (m, 2 H) 1.36-1.58 (m, $4 \mathrm{H}), 0.98$ (t, $J=7.1 \mathrm{~Hz}, 3 \mathrm{H}) \mathrm{ppm}$; HRMS (ESI) $\mathrm{m} / \mathrm{z} 558.2573\left[(\mathrm{M}+\mathrm{H})^{+}\right.$; calcd for $\mathrm{C}_{32} \mathrm{H}_{36} \mathrm{~N}_{3} \mathrm{O}_{6}$ : 558.2599].

Methyl (3R,5S,8S,15E)-8-butyl-7,10-dioxo-22-phenyl-2,11-dioxa-6,9,21triazatetracyclo[15.6.2.1 3,6 .0 20,24 ]hexacosa-1(23),15,17,19,21,24-hexaene-5-carboxylate (10c)

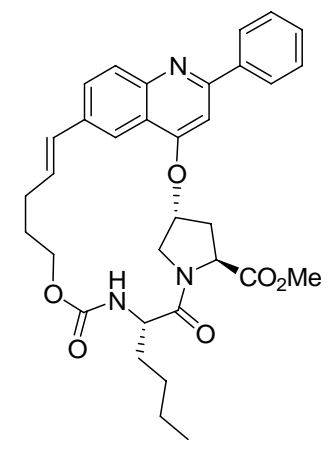

Prepared in the same manner as 10a (84\%). ${ }^{1} \mathrm{H}$ NMR (400 MHz, $\left.\mathrm{CDCl}_{3}\right) \delta 8.35$ (brs, $1 \mathrm{H}$ ), 8.29 (d, $J=$ 8.7 Hz, $1 \mathrm{H}$ ), 7.88-7.94 (m, $2 \mathrm{H}$ ), 7.72 (dd, $J=8.8,1.7 \mathrm{~Hz}, 1 \mathrm{H}), 7.32-7.38$ (m, $3 \mathrm{H}), 7.18$ (s, $1 \mathrm{H}), 6.53$ (d, $J=15.8 \mathrm{~Hz}, 1 \mathrm{H}), 6.49$ (m, $1 \mathrm{H}), 5.69(\mathrm{~m}, 1 \mathrm{H}), 5.29$ (d, $J=7.9 \mathrm{~Hz}, 1 \mathrm{H}), 4.88$ (d, $J=11.3 \mathrm{~Hz}, 1 \mathrm{H}$ ), 4.68-4.74 (m, 1 H), 4.43-4.57 (m, 2 H), 3.96-4.10 (m, 2 H), 3.75 (s, 3 H), 2.74 (dd, $J=7.6,13.6$ Hz, 1 H), 2.35-2.45 (m, $3 \mathrm{H}), 1.70-2.05(\mathrm{~m}, 4 \mathrm{H}), 1.37-1.56(\mathrm{~m}, 4 \mathrm{H}), 0.97$ (t, $J=7.1 \mathrm{~Hz}, 3 \mathrm{H})$ ppm; HRMS (ESI) $\mathrm{m} / \mathrm{z} 572.2749\left[(\mathrm{M}+\mathrm{H})^{+}\right.$; calcd for $\left.\mathrm{C}_{33} \mathrm{H}_{38} \mathrm{~N}_{3} \mathrm{O}_{6}: 572.2755\right]$. 
Methyl (3R,5S,8S,16E)-8-butyl-7,10-dioxo-23-phenyl-2,11-dioxa-6,9,22triazatetracyclo[16.6.2.1 3,6 .0 21,25 ]heptacosa-1(24),16,18,20,22,25-hexaene-5-carboxylate (10d)

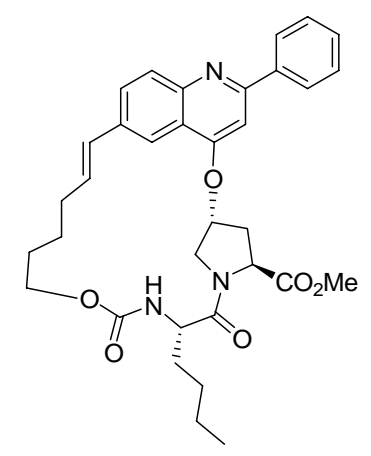

Prepared in the same manner as $10 \mathrm{a}(86 \%) .{ }^{1} \mathrm{H}$ NMR (400 $\mathrm{MHz}, \mathrm{CDCl}_{3}$ ) (mixture of Boc rotamers, major rotamer) $\delta 8.29(\mathrm{~d}, J=8.8 \mathrm{~Hz}, 1 \mathrm{H}), 8.14(\mathrm{~d}, J=1.7 \mathrm{~Hz}, 1 \mathrm{H}), 7.88-7.94(\mathrm{~m}, 2 \mathrm{H}), 7.76(\mathrm{dd}, J=$ 8.9, $1.8 \mathrm{~Hz}, 1 \mathrm{H}), 7.30-7.35$ (m, $3 \mathrm{H}), 7.17$ (s, $1 \mathrm{H}), 6.61$ (d, $J=15.9 \mathrm{~Hz}, 1 \mathrm{H}), 6.49$ (dt, $J=15.9,6.7 \mathrm{~Hz}$, $1 \mathrm{H}$ ), 5.73 (brt $1 \mathrm{H}$ ), 5.30 (d, $J=8.4 \mathrm{~Hz}, 1 \mathrm{H}$ ), 4.76 (d, $J=11.0 \mathrm{~Hz}, 1 \mathrm{H}$ ), 4.69 (dd, $J=9.5,8.0 \mathrm{~Hz}, 1 \mathrm{H}$ ), 4.55-4.64 (m, 2 H), 4.08 (dd, $J=12.0,3.3$ Hz, 1 H), 3.85 (dt, $J=11.0,7.1$ Hz, 1 H), 3.74 (s, 3 H), 2.68$2.75(\mathrm{~m}, 1 \mathrm{H}), 2.28-2.52(\mathrm{~m}, 3 \mathrm{H}), 1.35-1.90(\mathrm{~m}, 10 \mathrm{H0}, 0.97$ (t, $J=7.2 \mathrm{~Hz}, 3 \mathrm{H}) \mathrm{ppm}$; HRMS (ESI) m/z $586.2890\left[(\mathrm{M}+\mathrm{H})^{+}\right.$; calcd for $\mathrm{C}_{34} \mathrm{H}_{40} \mathrm{~N}_{3} \mathrm{O}_{6}$ : 586.2912].

\section{Ethyl (1R,2S)-1-amino-2-vinylcyclopropanecarboxylate (11)}<smiles>C=C/C(N)=C(/Cl)OCC</smiles>

11 was prepared as described previously (Beaulieu, P. L.; Gillard, J.; Bailey, M. D.; Boucher, C.; Duceppe, J.-S.; Simoneau, B.; Wang, X.-J.; Zhang, L.; Grozinger, K.; Houpis, I.; Farina, V.; Heimroth, H.; Krueger, T.; Schnaubelt, J. J. Org. Chem., 2005, 70, 5869-5879).

Methyl (1R,2S)-1-(\{[(3R,5S,8S)-8-butyl-7,10-dioxo-20-phenyl-2,11-dioxa-6,9,19triazatetracyclo[13.6.2.1 3,6 .0 18,22 ]tetracosa-1(21),15,17,19,22-pentaen-5-yl]carbonyl\}amino)2-vinylcyclopropanecarboxylate (12a)

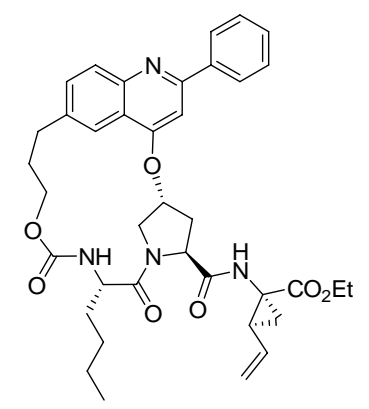

A solution of 10a (89 mg, $0.164 \mathrm{mmol})$ in EtOH (30 mL) was hydrogenated at $1 \mathrm{~atm}$. over 10\% Pd/C (20 mg) for $1 \mathrm{~h}$ at RT. The reaction mixture was filtered, the filtrate concentrated and crude product purified by reverse phase HPLC to afford $20 \mathrm{mg}$ of a foam (major product resulted from significant 
cleavage of the allyl carbamate in this unoptimized reaction). The foam was dissolved in THF (2 mL), $\mathrm{MeOH}(2 \mathrm{~mL})$ and water ( $2 \mathrm{~mL} 0$ and treated with $\mathrm{LiOH}$ ( $3.5 \mathrm{mg}, 0.147 \mathrm{mmol}$ ). The reaction mixture was stirred at room temperature for $18 \mathrm{~h}$, treated with $1 \mathrm{M} \mathrm{HCl}(147 \mu \mathrm{L})$ and the mixture concentrated to dryness. The solid residue was taken up in DMF $(2 \mathrm{~mL})$ and treated with ethyl (1R,2S)-1-amino-2vinylcyclopropanecarboxylate ( $8.7 \mathrm{mg}, 0.045 \mathrm{mmol}$ ), DIPEA (20 $\mu \mathrm{L}, 0.113 \mathrm{mmol})$, DMAP (2.3 mg, $0.019 \mathrm{mmol}$ ) and HATU (18.6 $\mathrm{mg}, 0.049 \mathrm{mmol})$. After $2 \mathrm{~h}$, the reaction mixture was purified directly by reverse phase HPLC to give the product (10 mg, 9\%). ${ }^{1} \mathrm{H}$ NMR $\left(400 \mathrm{MHz}, \mathrm{CDCl}_{3}\right) \delta 8.32$ (d, $J=8.5 \mathrm{~Hz}$, 1 H), 7.88-7.94 (m, 2 H), 7.74-7.82 (m, 2 H), 7.65 (s, 1 H), 7.50-7.58 (m, 3 H), 7.17 s, 1 H), 5.68-5.80 (m, $1 \mathrm{H}), 5.47-5.60$ (m, $2 \mathrm{H}), 5.31$ (d, $J=17.1 \mathrm{~Hz}, 1 \mathrm{H}), 5.15$ (d, $J=10.5 \mathrm{~Hz}, 2 \mathrm{H}), 4.85-4.94(\mathrm{~m}, 1 \mathrm{H})$, 4.66-4.75 (m, 1 H), 4.07-4.23 (m, 3 H), 3.81-3.90 (m, 2 H), 2.84-3.19 (m, 3 H), 2.44-2.54 (m, 1 H), 2.25-2.36 (m, $1 \mathrm{H}), 2.16$ (q, $J=8.7 \mathrm{~Hz}, 1 \mathrm{H}), 1.30-1.95$ (m, $9 \mathrm{H}), 1.23$ (t, $J=7.1 \mathrm{~Hz}, 3 \mathrm{H}$ ), 0.90-0.97 (m, $3 \mathrm{H}$ ) ppm; HRMS (ESI) $\mathrm{m} / z$ 669.3261 [(M+H) ${ }^{+}$; calcd for $\left.\mathrm{C}_{38} \mathrm{H}_{45} \mathrm{~N}_{4} \mathrm{O}_{7}: 669.3283\right]$.

Methyl (1R,2S)-1-(\{[(3R,5S,8S)-8-butyl-7,10-dioxo-21-phenyl-2,11-dioxa-6,9,20triazatetracyclo[14.6.2.1 3,6 .0 19,23 ]pentacosa-1(22),16,18,20,23-pentaen-5yl]carbonyl\}amino)-2-vinylcyclopropanecarboxylate (12b)

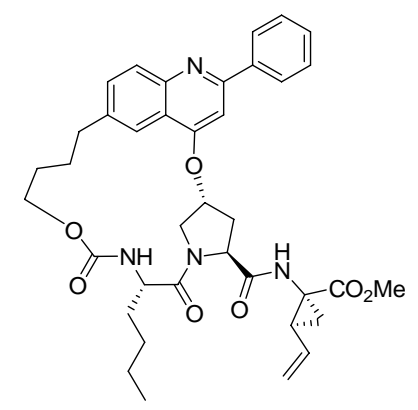

Prepared in the same manner as 12a (85\%). ${ }^{1} \mathrm{H} \mathrm{NMR}\left(400 \mathrm{MHz}, \mathrm{CDCl}_{3}\right) \delta 8.40(\mathrm{~d}, \mathrm{~J}=8.7 \mathrm{~Hz}, 1 \mathrm{H})$, 8.06 (brs, 1 H), 7.93-7.98 (m, 2 H), 7.76 (dd, J = 8.7, 1.8 Hz, 1 H), 7.62 (s, 1 H), 7.50-7.59 (m, 3 H), 7.09 (s, $1 \mathrm{H}$ ), 5.74 (ddd, $J=18.9,10.3,8.7 \mathrm{~Hz}, 1 \mathrm{H}$ ), 5.50 (t, $J=3.5 \mathrm{~Hz}, 1 \mathrm{H}$ ), 5.42 (d, $J=9.5 \mathrm{~Hz}, 1 \mathrm{H}$ ), 5.29 (dd, $J=17.1,1.0 \mathrm{~Hz}, 1 \mathrm{H}$ ), 5.13 (dd, $J=10.3,1.4 \mathrm{~Hz}, 1 \mathrm{H}), 4.81-4.94$ (m, $3 \mathrm{H}), 4.75$ (d, $J=12.1$ Hz, 1 H), 4.06-4.22 (m, 2 H), 3.81-3.90 (m, 2 H), 3.11-3.22 (m, 2 H), 2.66-2.75 (m, 1 H), 2.48 (dd, $J=$ 14.6, $8.5 \mathrm{~Hz}, 1 \mathrm{H}), 2.11$ (q, $J=8.7 \mathrm{~Hz}, 1 \mathrm{H}), 1.62-1.90$ (m, $7 \mathrm{H}), 1.33-1.48$ (m $5 \mathrm{H}), 1.22$ (t, $J=7.1 \mathrm{~Hz}$, $3 \mathrm{H}), 0.94(\mathrm{t}, J=7.1 \mathrm{~Hz}, 3 \mathrm{H}) \mathrm{ppm}$; HRMS (ESI) $\mathrm{m} / \mathrm{z} 683.3440\left[(\mathrm{M}+\mathrm{H})^{+}\right.$; calcd for $\mathrm{C}_{39} \mathrm{H}_{47} \mathrm{~N}_{4} \mathrm{O}_{7}$ : 683.3439]. 
Methyl (1R,2S)-1-(\{[(3R,5S,8S)-8-butyl-7,10-dioxo-22-phenyl-2,11-dioxa-6,9,21triazatetracyclo[15.6.2.1 3,6 .0 20,24 ]hexacosa-1(23),17,19,21,24-pentaen-5-yl]carbonyl\}amino)2-vinylcyclopropanecarboxylate (12c)

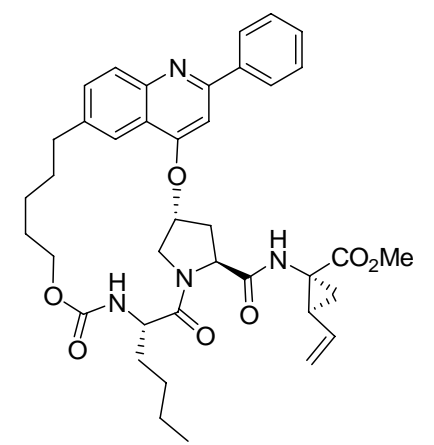

Prepared in the same manner as 12a (42\%). ${ }^{1} \mathrm{H} \mathrm{NMR}\left(400 \mathrm{MHz}, \mathrm{CDCl}_{3}\right) \delta 8.43(\mathrm{~d}, J=8.7 \mathrm{~Hz}, 1 \mathrm{H})$, 8.07 (brs, $1 \mathrm{H}$ ), 7.94-7.99 (m, $2 \mathrm{H}$ ), 7.78 (dd, $J=8.8,1.7$ Hz, $1 \mathrm{H}), 7.54-7.60$ (m, $4 \mathrm{H}), 7.15$ (s, $1 \mathrm{H}$ ), 5.70-5.80 (m, 1 ), 5.56 (brs, 1 H), 5.29 (d, $J=17.2$ Hz, 1 H), 5.23 (d, $J=8.3$ Hz, $1 \mathrm{H}$ ), 5.14 (dd, $J=$ 11.6, $1.4 \mathrm{~Hz}, 1 \mathrm{H}$ ), 4.89 (t, $J=8.1 \mathrm{~Hz}, 1 \mathrm{H}$ ), 4.82 (d, $J=12.0 \mathrm{~Hz}, 1 \mathrm{H}$ ), 4.55 (q, $J=7.5 \mathrm{~Hz}, 1 \mathrm{H}$ ), 3.854.35 (m, 5 H), 3.10-3.20 (m, $1 \mathrm{H}), 2.87-2.95$ (m, $1 \mathrm{H}), 2.74-2.84$ (m, $1 \mathrm{H}), 2.47$ (dd, $J=14.1,8.6 \mathrm{~Hz}, 1$ H), 2.13 (q, $J=8.7 \mathrm{~Hz}, 1 \mathrm{H}), 1.65-1.90(\mathrm{~m}, 6 \mathrm{H}), 1.30-1.50(\mathrm{~m}, 7 \mathrm{H}), 1.22(\mathrm{t}, J=7.1 \mathrm{~Hz}, 1 \mathrm{H}), 0.93(\mathrm{t}$, $J=7.0 \mathrm{~Hz}, 1 \mathrm{H}$ ) ppm; HRMS (ESI) $\mathrm{m} / \mathrm{z} 697.3559$ [(M+H) ${ }^{+}$; calcd for $\mathrm{C}_{40} \mathrm{H}_{49} \mathrm{~N}_{4} \mathrm{O}_{7}$ : 697.3596].

Methyl (1R,2S)-1-(\{[(3R,5S,8S)-8-butyl-7,10-dioxo-23-phenyl-2,11-dioxa-6,9,22triazatetracyclo[16.6.2.1 3,6 .0 21,25 ]heptacosa-1(24),18,20,22,25-pentaen-5yl]carbonyl\}amino)-2-vinylcyclopropanecarboxylate (12d)

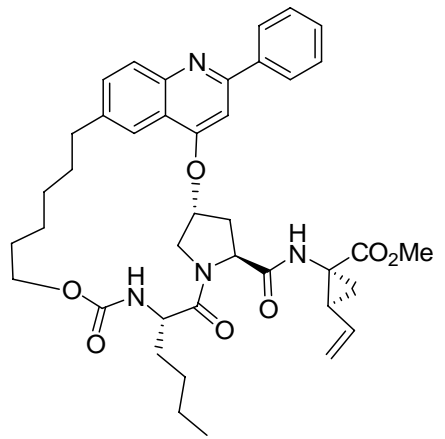

Prepared in the same manner as 12a. ${ }^{1} \mathrm{H}$ NMR (400 MHz, $\left.\mathrm{CDCl}_{3}\right) \delta 8.45$ (d, $\left.J=8.8 \mathrm{~Hz}, 1 \mathrm{H}\right)$, 7.97-8.02 (m, 2 H), 7.91 (brs, 1 H), 7.76 (dd, $J=8.8,1.7$ Hz, 1 H), 7.52-7.60 (m, 2 H), 7.50 (brs, 1 H), 7.13 (s, 1 H), 5.75 (ddd, $J=18.9,10.3,8.7$ Hz, 1 H), 5.54 (m, 1 H), 5.25-5.33 (m, 2 H), 5.14 (dd, $J=10.3,1.1$ Hz, $1 \mathrm{H}), 4.85$ (t, $J=7.8 \mathrm{~Hz}, 1 \mathrm{H}), 4.68(\mathrm{~d}, J=12.3 \mathrm{~Hz}, 1 \mathrm{H}), 4.60$ (q, $J=7.5 \mathrm{~Hz}, 1 \mathrm{H}), 4.36$ (dt, $J=10.9$, $6.8 \mathrm{~Hz}, 1 \mathrm{H}), 4.06-4.21$ (m, $2 \mathrm{H}), 3.97$ (dd, $J=12.3$, $3.6 \mathrm{~Hz}, 1 \mathrm{H}), 3.76$ (dt, $J=10.9,6.7 \mathrm{~Hz}, 1 \mathrm{H})$, 3.073.16 (m, $1 \mathrm{H}$ ), 2.71-2.90 (m, $2 \mathrm{H}$ ), 2.48 (dd, $J=14.7,8.5 \mathrm{~Hz}, 1 \mathrm{H}), 2.13$ (q, $J=8.8 \mathrm{~Hz}, 1 \mathrm{H}), 1.29-1.88$ (m, $16 \mathrm{H}), 1.22$ (t, $J=7.1 \mathrm{~Hz}, 3 \mathrm{H}), 0.92$ (, $J=7.1 \mathrm{~Hz}, 3 \mathrm{H}$ ) ppm; HRMS (ESI) m/z 711.3744 [(M+H) ${ }^{+}$; calcd for $\left.\mathrm{C}_{41} \mathrm{H}_{51} \mathrm{~N}_{4} \mathrm{O}_{7}: 711.3752\right]$. 
(1R,2S)-1-(\{[(3R,5S,8S)-8-Butyl-7,10-dioxo-20-phenyl-2,11-dioxa-6,9,19triazatetracyclo[13.6.2.1 3,6 .0 18,22 ]tetracosa-1(21),15,17,19,22-pentaen-5-yl]carbonyl\}amino)2-vinylcyclopropanecarboxylic acid (3a)

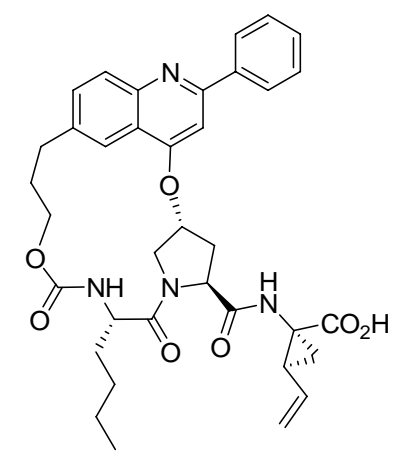

A solution of 12a (10 mg, $0.015 \mathrm{mmol})$ in THF (1 mL), MeOH (1 mL) and water (1 mL) at RT was treated with $\mathrm{LiOH}(1.5 \mathrm{mg}, 0.06 \mathrm{mmol})$ and stirred for $18 \mathrm{~h}$. $1 \mathrm{M} \mathrm{HCl}(60 \mu \mathrm{L})$ was added, the volatiles evaporated and the residue purified by reverse phase HPLC to afford the product (8mg, 80\%). ${ }^{1} \mathrm{H}$ NMR $\left(400 \mathrm{MHz}, \mathrm{CD}_{3} \mathrm{OD}\right) \delta 8.67$ (brs, $1 \mathrm{H}$ ), $8.16(\mathrm{~d}, J=8.7 \mathrm{~Hz}, 1 \mathrm{H}$ ), 8.06-8.11 (m, $2 \mathrm{H}$ ), 7.98 (dd, $J=8.7$, $1.9 \mathrm{~Hz}, 1 \mathrm{H}$ ), 7.80 (d, $J=1.6 \mathrm{~Hz}, 1 \mathrm{H}$ ), 7.70-7.80 (m, $4 \mathrm{H}$ ), 5.78-5.88 (m, $2 \mathrm{H}$ ), 5.29 (dd, $J=17.2,1.4$ Hz, 1 H), 5.09 (dt, $J=1.7,10.3 \mathrm{~Hz}, 2 \mathrm{H}), 4.61-4.68$ (m, $2 \mathrm{H}$ ), 3.97-4.08 (m, $2 \mathrm{H}), 3.76-3.82$ (m, $1 \mathrm{H}$ ), $3.11(\mathrm{dt}, J=3.5,13.2 \mathrm{~Hz}, 1 \mathrm{H}), 2.96(\mathrm{dt}, J=13.5,3.7 \mathrm{~Hz}, 1 \mathrm{H}), 2.77-2.86(\mathrm{~m}, 1 \mathrm{H}), 2.58-2.67(\mathrm{~m}, 1 \mathrm{H})$, 2.24-2.35 (m, $1 \mathrm{H}), 2.20$ (q, $J=8.8 \mathrm{~Hz}, 1 \mathrm{H}), 1.66-1.93(\mathrm{~m}, 4 \mathrm{H}), 1.28-1.47$ (m, $5 \mathrm{H}), 0.97$ (t, $J=7.0 \mathrm{~Hz}$, $3 \mathrm{H}$ ) ppm; HRMS (ESI) $\mathrm{m} / \mathrm{z} 641.2974\left[(\mathrm{M}+\mathrm{H})^{+}\right.$; calcd for $\left.\mathrm{C}_{36} \mathrm{H}_{41} \mathrm{~N}_{4} \mathrm{O}_{7}: 641.2970\right]$; $[\alpha]_{D}^{25}-180.7^{\circ}$ (c = $0.41, \mathrm{MeOH})$.

(1R,2S)-1-(\{[(3R,5S,8S)-8-Butyl-7,10-dioxo-21-phenyl-2,11-dioxa-6,9,20triazatetracyclo[14.6.2.1 3,6 .0 19,23 ]pentacosa-1(22),16,18,20,23-pentaen-5yl]carbonyl\}amino)-2-vinylcyclopropanecarboxylic acid (3b)

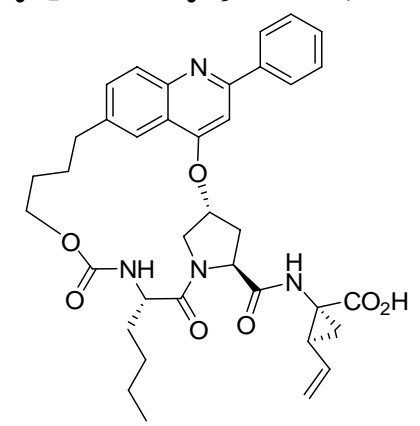

Prepared in the same manner as 3a (90\%). ${ }^{1} \mathrm{H}$ NMR $\left(400 \mathrm{MHz}, \mathrm{CD}_{3} \mathrm{OD}\right) \delta 8.01-8.07$ (m $\left.3 \mathrm{H}\right), 7.91$ (d, $J$ $=8.6 \mathrm{~Hz}, 1 \mathrm{H}), 7.48-7.60(\mathrm{~m}, 4 \mathrm{H}), 7.31(\mathrm{~s}, 1 \mathrm{H}), 7.23(\mathrm{~d}, J=9.1 \mathrm{~Hz}, 1 \mathrm{H}), 5.77-5.87(\mathrm{~m}, 1 \mathrm{H}), 5.48-5.53$ (m, $1 \mathrm{H}$ ), 5.27 (dd, $J=17.2,1.5 \mathrm{~Hz}, 1 \mathrm{H}), 5.08$ (dd, $J=10.3,1.7 \mathrm{~Hz}, 1 \mathrm{H}), 4.67-4.81$ (m, $3 \mathrm{H}), 4.50$ (t, $J$ $=8.7 \mathrm{~Hz}, 1 \mathrm{H}), 4.00$ (dd, $J=12.0,2.7 \mathrm{~Hz}, 1 \mathrm{H}), 3.75-3.83(\mathrm{~m}, 1 \mathrm{H}), 2.94-3.04(\mathrm{~m}, 1 \mathrm{H}), 2.7-2.86(\mathrm{~m}, 1$ H), 2.47 (ddd, $J=13.8,9.4,3.9 \mathrm{~Hz}, 1 \mathrm{H}), 2.17$ (q, $J=8.8 \mathrm{~Hz}, 1 \mathrm{H}), 1.56-1.90(\mathrm{~m}, 7 \mathrm{H}), 1.36-1.48$ (m, 5 $\mathrm{H}), 0.97(\mathrm{t}, J=7.0 \mathrm{~Hz}, 3 \mathrm{H}) \mathrm{ppm}$; HRMS (ESI) $\mathrm{m} / \mathrm{z} 655.3109\left[(\mathrm{M}+\mathrm{H})^{+}\right.$; calcd for $\mathrm{C}_{37} \mathrm{H}_{43} \mathrm{~N}_{4} \mathrm{O}_{7}$ : 655.3126]; $[\alpha]_{D}^{25}-134.4^{\circ}(\mathrm{c}=0.44, \mathrm{MeOH})$. 
(1R,2S)-1-(\{[(3R,5S,8S)-8-Butyl-7,10-dioxo-22-phenyl-2,11-dioxa-6,9,21triazatetracyclo[15.6.2.1 3,6 .0 20,24 ]hexacosa-1(23),17,19,21,24-pentaen-5-yl]carbonyl\}amino)2-vinylcyclopropanecarboxylic acid (3c)

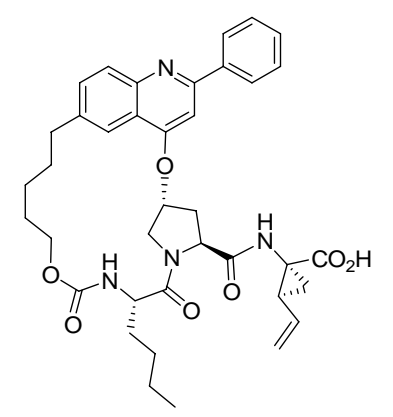

Prepared in the same manner as 3a (87\%). ${ }^{1} \mathrm{H}$ NMR $\left(500 \mathrm{MHz}, \mathrm{CDCl}_{3}\right) \delta 8.68(\mathrm{~s}, 1 \mathrm{H}), 8.22(\mathrm{~s}, 1 \mathrm{H})$, 8.14 (d, $J=8.5 \mathrm{~Hz}, 1 \mathrm{H}$ ), 8.06-8.12 (m, $2 \mathrm{H}$ ), 7.98 (dd, $J=8.6,1.8 \mathrm{~Hz}, 1 \mathrm{H}$ ), 7.81 (s, $1 \mathrm{H}$ ), 7.70-7.80 (m, $3 \mathrm{H}$ ), 5.98 (brs, $1 \mathrm{H}$ ), 5.84 (dt, $J=19.0,10.0 \mathrm{~Hz}, 1 \mathrm{H}$ ), 5.28 (dd, $J=17.1,1.7 \mathrm{~Hz}, 1 \mathrm{H}$ ), 5.10 (dd, $J$ $=10.5,1.7 \mathrm{~Hz}, 1 \mathrm{H}), 4.64(\mathrm{dd}, J=10.0,7.7 \mathrm{~Hz}, 1 \mathrm{H}), 4.43(\mathrm{t}, J=7.6 \mathrm{~Hz}, 1 \mathrm{H}), 4.26(\mathrm{dt}, J=10.4,7.8$ $\mathrm{Hz}, 1 \mathrm{H}$ ), 4.11 (dd, $J=12.2,2.9 \mathrm{~Hz}, 1 \mathrm{H}), 2.50-3.05$ (m, $4 \mathrm{H}), 2.15-2.25$ (m, $1 \mathrm{H}), 1.50-1.95$ (m, $7 \mathrm{H}$ ), 1.22-1.50 (m, $7 \mathrm{H}), 0.97(\mathrm{t}, J=7.1 \mathrm{~Hz}, 3 \mathrm{H})$, ppm; HRMS (ESI) $m / z 669.3285$ [(M+H) ${ }^{+}$; calcd for $\mathrm{C}_{38} \mathrm{H}_{45} \mathrm{~N}_{4} \mathrm{O}_{7}$ : 669.3283]; $[\alpha]_{D}^{25}-76.7^{\circ}(\mathrm{c}=0.30, \mathrm{MeOH})$.

(1R,2S)-1-(\{[(3R,5S,8S)-8-Butyl-7,10-dioxo-23-phenyl-2,11-dioxa-6,9,22triazatetracyclo[16.6.2.1 3,6 .0 21,25 ]heptacosa-1(24),18,20,22,25-pentaen-5yl]carbonyl\}amino)-2-vinylcyclopropanecarboxylic acid (3d)

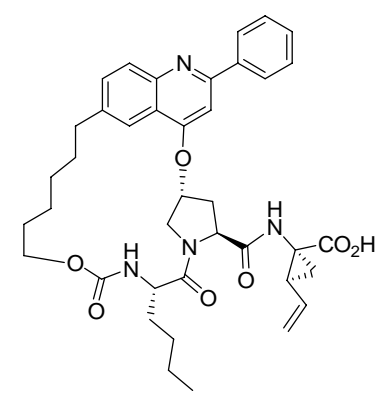

Prepared in the same manner as 3a (90\%). ${ }^{1} \mathrm{H}$ NMR (400 MHz, $\left.\mathrm{CD}_{3} \mathrm{OD}\right) \delta 8.67$ (s, $\left.1 \mathrm{H}\right)$, 8.06-8.18 (m, 4 H), 7.97 (dd, $J=8.7,1.8 \mathrm{~Hz}, 1 \mathrm{H}$ ), 7.70-7.80 (m, $4 \mathrm{H}$ ), 5.78-5.93 (m, $2 \mathrm{H}), 5.28$ (dd, $J=17.1,1.6 \mathrm{~Hz}, 1$ H), 5.09 (dd, $J=10.4,1.7 \mathrm{~Hz}, 1 \mathrm{H}), 4.68(\mathrm{~d}, J=12.2 \mathrm{~Hz}, 1 \mathrm{H}), 4.60(\mathrm{dd}, J=9.8,7.6 \mathrm{~Hz}, 1 \mathrm{H}), 4.37$ (t, $J$ $=7.2 \mathrm{~Hz}, 1 \mathrm{H}$ ), 4.10-4.20 (m, $2 \mathrm{H}$ ), 3.65 (ddd, $J=11.8,6.9,4.8 \mathrm{~Hz}, 1 \mathrm{H}$ ), 2.78-2.96 (m, $3 \mathrm{H}$ ), 2.57 (ddd, $J=14.3,10.0,3.9 \mathrm{~Hz}, 1 \mathrm{H}), 2.19$ (q, $J=8.8 \mathrm{~Hz}, 1 \mathrm{H}), 1.30-1.88$ (m, $16 \mathrm{H}), 0.96$ (t, $J=6.9 \mathrm{~Hz}, 3 \mathrm{H}) \mathrm{ppm}$; HRMS (ESI) $\mathrm{m} / \mathrm{z} 683.3423\left[(\mathrm{M}+\mathrm{H})^{+}\right.$; calcd for $\left.\mathrm{C}_{39} \mathrm{H}_{47} \mathrm{~N}_{4} \mathrm{O}_{7}: 683.3439\right]$; $[\alpha]_{D}^{25}-56.4^{\circ}$ (c = 0.33, MeOH). 
Synthesis of (1R,2S)-1-(\{[(3R,5S,8S)-8-Butyl-7,10-dioxo-23-phenyl-2,11-dioxa-6,9,22triazatetracyclo[15.7.1.1 3,6 .0 21,25 ]hexacosa-1(24),17(25),18,20,22-pentaen-5yl]carbonyl\}amino)-2-vinylcyclopropanecarboxylic acid (13)<smiles>C=CC(=O)NC(=O)C1CC(Oc2cc(-c3ccccc3)nc3cccc(CCCCCOC(=O)O)c23)CN1C(=O)[C@H](CCCC)NC(=O)C=C</smiles>

Step 1: 5-Bromo-2-phenylquinolin-4-ol (13_1)<smiles></smiles>

A mixture of 3 bromoaniline (17.6 g, $103 \mathrm{mmol})$, ethyl benzoylacetate (19.8 g, $103 \mathrm{mmol})$ and polyphosphoric acid $(70 \mathrm{~g})$ was heated to $150{ }^{\circ} \mathrm{C}$ for $2 \mathrm{~h}$, cooled and additional ethyl benzoylacetate (19.8 g, $103 \mathrm{mmol}$ ) added and heating at $150{ }^{\circ} \mathrm{C}$ continued for $2 \mathrm{~h}$. After allowing to cool slightly, the crude reaction mixture was poured into $6 \mathrm{M} \mathrm{HCl}(300 \mathrm{~mL})$, stirred for $30 \mathrm{~min}$, filtered and the solid washed with water $(50 \mathrm{~mL})$.The solid was suspended in $2 \mathrm{M} \mathrm{NaOH}(1000 \mathrm{~mL})$, THF (100 mL) added and the mixture heated to $80{ }^{\circ} \mathrm{C}$. Decolorizing charcoal (10 g) was added, the mixture stirred for $10 \mathrm{~min}$, filtered and the $\mathrm{pH}$ of the filtrate adjusted to 4.8 with acetic acid. The solid was filtered off and purified by chromatography on silica (5-20\% acetone /DCM) to give $1.9 \mathrm{~g}$ pure product together with mixed fractions. ${ }^{1} \mathrm{H}$ NMR (400 MHz, DMSO) $\delta 11.68$ (s, $1 \mathrm{H}$ ), 7.80-7.85 (m, $2 \mathrm{H}$ ), 7.76 (dd, $J=8.1,1.2 \mathrm{~Hz}, 1$ H), 7.57-7.62 (m, $3 \mathrm{H}), 7.52$ (dd, $J=7.7,1.1 \mathrm{~Hz}, 1 \mathrm{H}), 7.47$ (t, $J=7.9 \mathrm{~Hz}, 1 \mathrm{H}), 6.33$ (d, $J=2.0 \mathrm{~Hz}, 1$ H) ppm; HRMS (ESI) $\mathrm{m} / \mathrm{z} 300.0021$ [(M+H) ${ }^{+}$; calcd for $\mathrm{C}_{15} \mathrm{H}_{11} \mathrm{BrNO}$ : 300.0019].

Step 2: 1-t-Butyl 2-methyl (2S,4R)-4-[(5-bromo-2-phenylquinolin-4-yl)oxy]pyrrolidine-1,2dicarboxylate (13_2)<smiles>CCOC(=O)N1CC(Oc2ccccc2)C(=O)O1</smiles>

To a suspension of 13_1 (515 mg, $1.72 \mathrm{mmol})$ and 1-tert-butyl 2-methyl (2S, 4S)-4-\{[(4bromophenyl)sulfonyl] oxy\} pyrrolidine-1,2-dicarboxylate (Arasappan, A. et al, J. Org. Chem. 2002, 67, 3923-3926) (956 mg, $2.06 \mathrm{mmol}$ ) in $\mathrm{N}$-methylpyrrolidinone (4 mL) was added $\mathrm{Cs}_{2} \mathrm{CO}_{3}$ (839 mg, 2.57 $\mathrm{mmol}$ ) and the mixture heated at $45^{\circ} \mathrm{C}$. After $3 \mathrm{~h}$, the mixture was partitioned between water and 
EtOAc. The organic phase was washed with brine, dried over anhydrous $\mathrm{Na}_{2} \mathrm{SO}_{4}$ and solvent evaporated. The crude product was purified by chromatography on silica (30-100\% EtOAc hexane) to afford 13_2 (820 mg, 91\%). ${ }^{1} \mathrm{H}$ NMR $\left(400 \mathrm{MHz}, \mathrm{CD}_{3} \mathrm{OD}\right)(\sim 2: 1$ mixture of Boc rotamers, major rotamer) $\delta 8.13-8.17(\mu, 1 \mathrm{H}), 8.04-8.10(\mathrm{~m}, 3 \mathrm{H}), 7.74-7.80(\mathrm{~m}, 1 \mathrm{H})$, 7.64-7.72 (m, $4 \mathrm{H}), 5.73(\mathrm{~m}, 1$ H), 4.57-64 (m, $1 \mathrm{H}), 4.14-4.18(\mathrm{~m}, 1 \mathrm{H}), 3.79-3.88(\mathrm{~m}, 1 \mathrm{H}), 3.78(\mathrm{~s}, 3 \mathrm{H}), 2.82-2.93(\mathrm{~m}, 1 \mathrm{H}), 2.36-$ $2.48(\mathrm{~m}, 1 \mathrm{H}), 1.44(\mathrm{~s}, 9 \mathrm{H}) \mathrm{ppm}$; HRMS (ESI) $\mathrm{m} / \mathrm{z} 527.1182\left[(\mathrm{M}+\mathrm{H})^{+}\right.$; calcd for $\mathrm{C}_{26} \mathrm{H}_{28} \mathrm{BrN}_{2} \mathrm{O}_{5}$ : 527.1176].

Step 3: Methyl N-[(pent-4-en-1-yloxy)carbonyl]-L-norleucyl-(4R)-4-[(5-bromo-2-phenylquinolin-4yl)oxy]-L-prolinate (13_3)

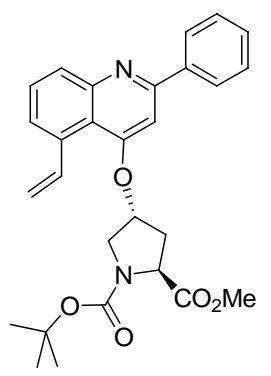

A solution of 13_2 (600 mg, $1.138 \mathrm{mmol})$ in toluene $(10 \mathrm{~mL})$ was degassed by bubbling nitrogen through for $30 \mathrm{~min}$ and then treated with vinyltributyltin $(401 \mu \mathrm{L}, 1.365 \mathrm{mmol})$ and tetrakis(triphenylphosphine)palladium(0) (131 mg, $0.114 \mathrm{mmol}$ ). The mixture was then heated to 105 oC for $3 \mathrm{~h}$, cooled, concentrated and purified by chromatography on silica (25-60\% EtOAc hexane) to give 13_3 as a foam (480 mg, 89\%). ${ }^{1} \mathrm{H}$ NMR (400 MHz, $\mathrm{CD}_{3} \mathrm{OD}$ ) (mixture of Boc rotamers, major rotamer) $\delta 8.13(\mathrm{~d}, J=8.5 \mathrm{~Hz}, 1 \mathrm{H}), 7.99-8.06(\mathrm{~m}, 3 \mathrm{H}), 7.66-7.78(\mathrm{~m}, 5 \mathrm{H}), 7.55$ (dd, $J=17.2,10.9 \mathrm{~Hz}$, $1 \mathrm{H}), 5.75-5.82(\mathrm{~m}, 1 \mathrm{H}), 5.56$ (d, $J=10.9 \mathrm{~Hz}, 1 \mathrm{H}), 5.40(\mathrm{~d}, J=17.3 \mathrm{~Hz}, 1 \mathrm{H}), 4.46-4.53(\mathrm{~m}, 1 \mathrm{H}), 4.07$ (dd, $J=12.8,2.0 \mathrm{~Hz}, 1 \mathrm{H}$ ), 3.78-3.88 (m, $1 \mathrm{H}$ ), 3.76 (s, $3 \mathrm{H}$ ), 2.78-2.90 (m, $1 \mathrm{H}), 2.36-2.48$ (m, $1 \mathrm{H}$ ), 1.41 (s, 9 H) ppm; HRMS (ESI) $\mathrm{m} / \mathrm{z} 475.2228$ [(M+H) ${ }^{+}$; calcd for $\mathrm{C}_{28} \mathrm{H}_{31} \mathrm{~N}_{2} \mathrm{O}_{5}$ : 475.2228].

Step 4: Methyl N-[(pent-4-en-1-yloxy)carbonyl]-L-norleucyl-(4R)-4-[(2-phenyl-5-vinylquinolin-4yl)oxy]-L-prolinate (13_4)

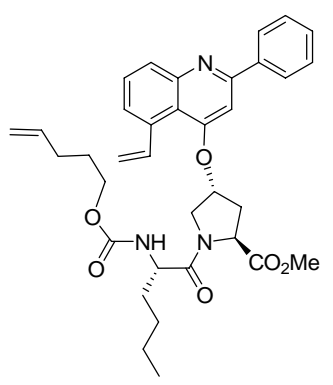

A solution of 13_3 (430 mg, $0.906 \mathrm{mmol})$ in 4M HCL/dioxane $(10 \mathrm{~mL})$ was stirred at RT for $3 \mathrm{~h}$, then the volatiles evaporated to a foam which was dissolved in DMF (4 mL). DIPEA ( $446 \mu \mathrm{L}, 2.56 \mathrm{mmol}$ ), 8c (228 mg, $0.937 \mathrm{mmol}$ ), DMAP (52 mg, $0.426 \mathrm{mmol}$ ) and HATU (421 mg, $1.107 \mathrm{mmol}$ ) were added and the reaction mixture stirred for $18 \mathrm{~h}$ at RT. The mixture was then partitioned between water and EtOAc (2) and the extracts washed with water, pH5.2 citrate buffer and saturated $\mathrm{NaHCO}_{3}$. The organic layer was dried over anhydrous $\mathrm{Na} 2 \mathrm{SO} 4$, the solvent evaporated and the residue purified by silica gel 
chromatography (30-80\% EtOAc hexane) to give 13_4 (376 mg, 74\%). ${ }^{1} \mathrm{H}$ NMR (400 MHz, $\left.\mathrm{CDCl}_{3}\right)$ (mixture of amide rotamers, major rotamer) $\delta 8.06(\mathrm{~m}, 3 \mathrm{H}), 7.62(\mathrm{t}, J=7.7 \mathrm{~Hz}, 1 \mathrm{H}), 7.44-7.54(\mathrm{~m}, 5$ H), 7.41 (d, $J=7.1 \mathrm{~Hz}, 1 \mathrm{H}$ ), 7.09 (s, $1 \mathrm{H}), 5.72-5.84$ (m, $1 \mathrm{H}$ ), 5.45 (d, $J=8.1 \mathrm{~Hz}, 1 \mathrm{H}), 5.33-5.40$ (m, 2 H), 5.18 (dd, $J=10.7,1.7 \mathrm{~Hz}, 1 \mathrm{H}$ ), 4.94-5.06 (m $2 \mathrm{H}), 4.69$ (t, $J=8.5 \mathrm{~Hz}, 1 \mathrm{H}), 4.50-4.56(\mathrm{~m}, 1 \mathrm{H})$, 4.38 (d, $J=11.5 \mathrm{~Hz}, 1 \mathrm{H}), 3.88-4.10$ (m, $3 \mathrm{H}), 3.76$ (s, $3 \mathrm{H}), 2.73-2.80$ (m, $1 \mathrm{H}), 2.28-2.35$ (m, $1 \mathrm{H}$ ), 2.04-2.15 (m, 3 H), 1.60-1.88 (m, 6 H), 1.20-1.50 (m, 5 H), 0.93 (m, 3 H) ppm; HRMS (ESI) m/z $600.3101\left[(\mathrm{M}+\mathrm{H})^{+}\right.$; calcd for $\left.\mathrm{C}_{35} \mathrm{H}_{42} \mathrm{~N}_{3} \mathrm{O}_{6}: 600.3068\right]$.

Step 5: Methyl (3R,5S,8S,15E)-8-butyl-7,10-dioxo-23-phenyl-2,11-dioxa-6,9,22triazatetracyclo[15.7.1.1 3,6 .0 21,25 ]hexacosa-1(24),15,17(25),18,20,22-hexaene-5-carboxylate (13_5)

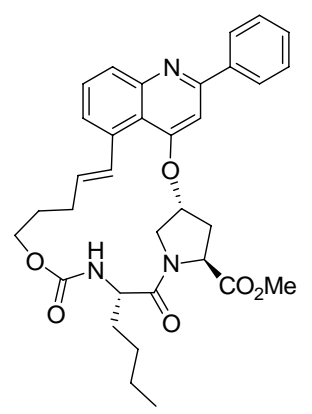

Prepared in the same manner as 10a (67\%). ${ }^{1} \mathrm{H}$ NMR (400 MHz, $\left.\mathrm{CDCl}_{3}\right) \delta 8.06(\mathrm{~d}, J=7.3 \mathrm{~Hz}, 2 \mathrm{H})$, 8.03 (d, $J=8.3 \mathrm{~Hz}, 1 \mathrm{H}$ ), 7.63 (brt, $J=7.4 \mathrm{~Hz}, 1 \mathrm{H}$ ), 7.45-7.56 (m, $6 \mathrm{H}$ ), 7.11 (s, $1 \mathrm{H}), 5.88-6.00$ (m, 1 H) 5.40-5.50 (m, 1 H), 4.30-4.85 (m, 3 H), 4.004 .30 (m, 2 H), 3.77 (s, 3 H), 2.20-75 (m, 3 H), 1.30-1.95 (m, $7 \mathrm{H}$ ), 0.92-0.98 (m, $3 \mathrm{H})$ ppm; HRMS (ESI) $\mathrm{m} / \mathrm{z} 572.2758\left[(\mathrm{M}+\mathrm{H})^{+}\right.$; calcd for $\mathrm{C}_{33} \mathrm{H}_{38} \mathrm{~N}_{3} \mathrm{O}_{6}$ : 572.2755].

Step 6: Ethyl (1R,2S)-1-(\{[(3R,5S,8S)-8-butyl-7,10-dioxo-23-phenyl-2,11-dioxa-6,9,22triazatetracyclo[15.7.1.1 3,6 .0 21,25 ]hexacosa-1(24),17(25),18,20,22-pentaen-5yl]carbonyl\}amino)-2-vinylcyclopropanecarboxylate (13_6)

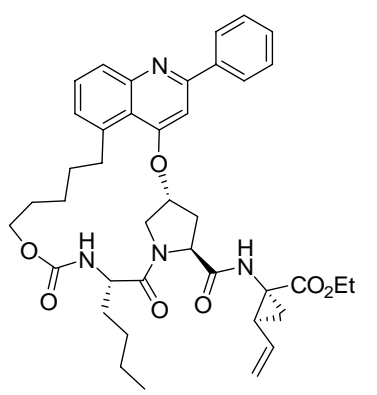

Prepared in the same manner as 12a (67\%). ${ }^{1} \mathrm{H}$ NMR $\left(400 \mathrm{MHz}, \mathrm{CDCl}_{3}\right) \delta 8.45(\mathrm{~d}, J=8.0 \mathrm{~Hz}, 1 \mathrm{H})$, 8.00 (d, $J=6.5 \mathrm{~Hz}, 2 \mathrm{H}$ ), 7.83 (dd, $J=8.5,7.3 \mathrm{~Hz}, 1 \mathrm{H}$ ), 7.53-7.63 (m, $4 \mathrm{H}$ ), 7.50 (d, $J=7.2 \mathrm{~Hz}, 1 \mathrm{H}$ ), 7.37 (brs, $1 \mathrm{H}$ ), 5.93 (brs, $1 \mathrm{H}$ ), 5.68-5.80 (m, $1 \mathrm{H}$ ), 5.31 (dd, $J=17.2,0.9 \mathrm{~Hz}, 1 \mathrm{H}$ ), 5.15 (dd, $J=10.2$, $1.2 \mathrm{~Hz}, 1 \mathrm{H}), 4.95-5.10$ (m, 2 H), 3.90-4.40 (m, 6 H), 3.10-3.35 (m, 3 H), 2.00-2.20 (m, 2 H), 1.30-1.90 (m, $12 \mathrm{H}), 1.21$ (t, $J=7.1 \mathrm{~Hz}, 3 \mathrm{H}$ ), 0.93 (t, $J=7.1 \mathrm{~Hz}, 3 \mathrm{H}$ ), ppm; HRMS (ESI) $\mathrm{m} / \mathrm{z} 697.3560$ $\left[(\mathrm{M}+\mathrm{H})^{+}\right.$; calcd for $\left.\mathrm{C}_{40} \mathrm{H}_{49} \mathrm{~N}_{4} \mathrm{O}_{7}: 697.3596\right]$. 
Step 7: (1R,2S)-1-(\{[(3R,5S,8S)-8-Butyl-7,10-dioxo-23-phenyl-2,11-dioxa-6,9,22-

triazatetracyclo[15.7.1.1 3,6 .0 21,25 ]hexacosa-1(24),17(25),18,20,22-pentaen-5-

yl]carbonyl\}amino)-2-vinylcyclopropanecarboxylic acid (13)

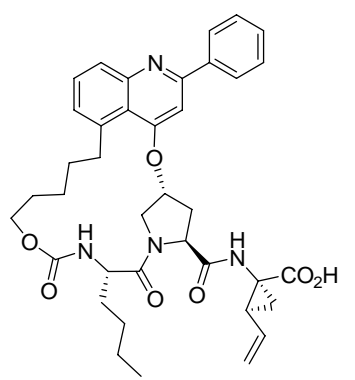

Prepared in the same manner as 3a. ${ }^{1} \mathrm{H}$ NMR $\left(400 \mathrm{MHz}, \mathrm{CD}_{3} \mathrm{OD}\right.$ ) (mixture of conformers, major conformer) $\delta 7.41$ (s, $1 \mathrm{H}), 8.04-8.11(\mathrm{~m}, 3 \mathrm{H}), 7.96$ (dd, $J=7.3,8.4 \mathrm{~Hz}, 1 \mathrm{H}), 7.62-7.77(\mathrm{~m}, 5 \mathrm{H}), 6.01$ (brs, $1 \mathrm{H}$ ), 5.84 (ddd, $J=19.1,10.3,9.0 \mathrm{~Hz}, 1 \mathrm{H}$ ), 5.26 (d, $J=17.0 \mathrm{~Hz}, 1 \mathrm{H}$ ), 5.07 (dd, $J=10.3,1.3 \mathrm{~Hz}$, $1 \mathrm{H}$ ), 4.66 (t, $J=7.5 \mathrm{~Hz}, 1 \mathrm{H}$ ), 4.20-4.40 (m, $4 \mathrm{H}), 3.52-3.78$ (m, $2 \mathrm{H}), 3.06-3.18$ (m, $1 \mathrm{H}), 2.60-2.70$ (m, $2 \mathrm{H}$ ), 2.20 (q, $J=8.8 \mathrm{~Hz}, 1 \mathrm{H}$ ), 1.30-1.85 (m, $13 \mathrm{H}), 0.93$ (t, $J=6.9 \mathrm{~Hz}, 3 \mathrm{H}$ ) ppm; HRMS (ESI) $\mathrm{m} / \mathrm{z}$ $669.3283\left[(\mathrm{M}+\mathrm{H})^{+}\right.$; calcd for $\left.\mathrm{C}_{38} \mathrm{H}_{45} \mathrm{~N}_{4} \mathrm{O}_{7}: 669.3283\right]$; $[\alpha]_{D}^{25}-8.21^{\circ}(\mathrm{c}=0.34, \mathrm{MeOH})$.

Synthesis of N-(Propoxycarbonyl)-L-norleucyl-(4R)-N-[(1R,2S)-1-carboxy-2-vinylcyclopropyl]-4[(6-ethyl-2-phenylquinolin-4-yl)oxy]-L-prolinamide (14)

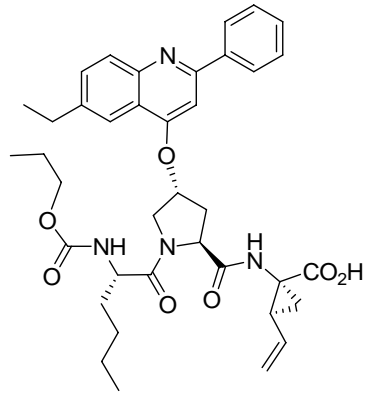

Step 1: N-(Propoxycarbonyl)-L-norleucyl-(4R)-N-[(1R,2S)-1-(ethoxycarbonyl)-2vinylcyclopropyl]-4-[(6-ethyl-2-phenylquinolin-4-yl)oxy]-L-prolinamide (14_1)

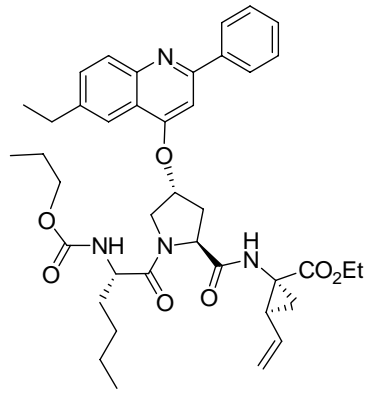

To a solution of $9 \mathbf{a}(88 \mathrm{mg}, 0.154 \mathrm{mmol})$ in THF $(10 \mathrm{~mL})$ was added $10 \% \mathrm{Pd} / \mathrm{C}(25 \mathrm{mg})$ and the mixture hydrogenated under a balloon of $\mathrm{H}_{2}$ for $3 \mathrm{~h}$. The catalyst was removed by filtration and water ( $5 \mathrm{~mL}$ ), $\mathrm{MeOH}(2.5 \mathrm{~mL}$ ) and $\mathrm{LiOH}(67 \mathrm{mg}, 2.8 \mathrm{mmol}$ ) added and the reaction mixture stirred ar RT for $1 \mathrm{~h}$, then $1 \mathrm{M} \mathrm{HCl}(2.7 \mathrm{~mL})$ added and the mixture evaporated. The solid residue was taken up in DMF ( $3 \mathrm{~mL})$ and treated with ethyl (1R,2S)-1-amino-2-vinylcyclopropanecarboxylate (37 mg, $0.192 \mathrm{mmol}$ ), DIPEA (81 
$\mu \mathrm{L}, 0.462 \mathrm{mmol}$ ), DMAP (9.4 $\mathrm{mg}, 0.077 \mathrm{mmol}$ ) and HATU (73 $\mathrm{mg}, 0.192 \mathrm{mmol}$ ). After $18 \mathrm{~h}$, the reaction mixture was purified directly by reverse phase HPLC to give the product (56 mg, 52\%). ${ }^{1} \mathrm{H}$ NMR (400 MHz, $\mathrm{CDCl}_{3}$ ) (mixture of amide rotamers, major rotamer) $\delta 8.06-8.11(\mathrm{~m}, 2 \mathrm{H}), 8.03(\mathrm{~d}, J=$ 8.6 Hz, $1 \mathrm{H}), 7.88$ (brs, $1 \mathrm{H})$, 7.56-7.60 (m, $1 \mathrm{H})$, 7.42-7.55 (m, $5 \mathrm{H}), 7.17$ (s, $1 \mathrm{H})$, 5.70-5.82 (m, $1 \mathrm{H})$, 5.44-5.52 (m, $1 \mathrm{H}), 5.26-5.36$ (m, $2 \mathrm{H}), 5.14$ (dd, $J=10.3,1.2 \mathrm{~Hz}, 1 \mathrm{H}), 4.87$ (dd, $J=8.2,4.7 \mathrm{~Hz}, 1 \mathrm{H})$, 4.50-4.56 (m, 1 H), 3.85-4.30 (m, 6 H), 3.02 (dt, $J=13.6,5.3$ Hz, 1 H), 2.80-2.90 (m, 2 H), 2.34-2.44 (m, $1 \mathrm{H}), 2.15$ (q, $J=8.8 \mathrm{~Hz}, 1 \mathrm{H}), 1.74-1.90$ (m, $2 \mathrm{H}), 1.52-1.70$ (m, $4 \mathrm{H}), 1.47$ (dd, $J=9.6,5.5 \mathrm{~Hz}, 1$ $\mathrm{H}), 1.25-1.45$ (m, $3 \mathrm{H}), 1.34$ (t, $J=7.6 \mathrm{~Hz}, 3 \mathrm{H}), 1.23$ (t, $J=7.6 \mathrm{~Hz}, 3 \mathrm{H}), 0.86-0.94$ (m, $6 \mathrm{H}) \mathrm{ppm}$; HRMS (ESI) $\mathrm{m} / \mathrm{z} 699.3759\left[(\mathrm{M}+\mathrm{H})^{+}\right.$; calcd for $\mathrm{C}_{40} \mathrm{H}_{51} \mathrm{~N}_{4} \mathrm{O}_{7}$ : 699.3752].

Step 2: N-(Propoxycarbonyl)-L-norleucyl-(4R)-N-[(1R,2S)-1-carboxy-2-vinylcyclopropyl]-4-[(6ethyl-2-phenylquinolin-4-yl)oxy]-L-prolinamide (14)

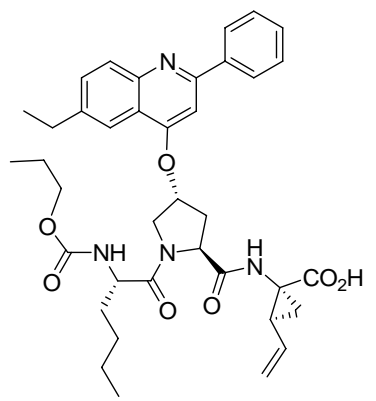

Prepared in the same manner as 3a. ${ }^{1} \mathrm{H}$ NMR (400 MHz, $\left.\mathrm{CD}_{3} \mathrm{OD}\right)$ (mixture of rotamers, major rotamer) $\delta 8.71(\mathrm{~s}, 1 \mathrm{H}), 8.21$ (s, $1 \mathrm{H}), 7.99-8.17$ (m, 4 H), 7.68-7.81 (m, 4 H), 5.78-5.90 (m, $2 \mathrm{H}), 5.29$ (d, $J=$ $17.2 \mathrm{~Hz}, 1 \mathrm{H}), 5.10$ (dd, $J=10.3,1.4 \mathrm{~Hz}, 1 \mathrm{H}), 4.66-4.78$ (m, $2 \mathrm{H}), 4.31$ (t, $J=7.1 \mathrm{~Hz}, 1 \mathrm{H}), 4.11$ (dd, $J$ = 12.2, $3.2 \mathrm{~Hz}, 1 \mathrm{H}$ ), 3.65 (dt, $J=10.4,6.7 \mathrm{~Hz}, 1 \mathrm{H}), 3.50$ (dt, $J=10.4,6.7 \mathrm{~Hz}, 1 \mathrm{H}), 2.95$ (q, $J=7.5 \mathrm{~Hz}$, $1 \mathrm{H}), 2.85$ (dd, $J=14.3,7.7 \mathrm{~Hz}, 1 \mathrm{H}), 2.60$ (ddd, $J=13.8,9.2,4.2 \mathrm{~Hz}, 1 \mathrm{H}), 2.22$ (q, $J=8.8 \mathrm{~Hz}, 1 \mathrm{H}$ ), 1.56-1.80 (m, $3 \mathrm{H}), 1.30-1.50$ (m, $9 \mathrm{H}), 0.93$ (t, $J=6.9 \mathrm{~Hz}, 3 \mathrm{H}), 0.80$ (t, $J=7.4 \mathrm{~Hz}, 3 \mathrm{H}) \mathrm{ppm}$; HRMS (ESI) $m / z$ 671.3425 [(M+H) ${ }^{+}$; calcd for $\mathrm{C}_{38} \mathrm{H}_{47} \mathrm{~N}_{4} \mathrm{O}_{7}$ : 671.3439]; $[\alpha]_{D}^{25}-30.9^{\circ}$ (c =0.31, MeOH).

(3R,5S,8S)-8-Butyl-N-((1R,2S)-1-\{[(cyclopropylsulfonyl)amino]carbonyl\}-2-vinylcyclopropyl)7,10-dioxo-22-phenyl-2,11-dioxa-6,9,21-triazatetracyclo[15.6.2.1 3,6 .0 20,24 ]hexacosa1(23),17,19,21,24-pentaene-5-carboxamide (15)

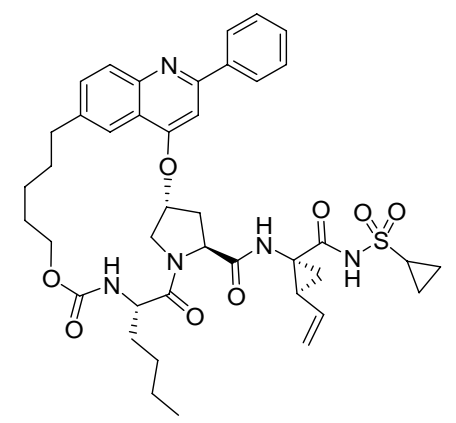

Prepared in the same manner as 12c. ${ }^{1} \mathrm{H}$ NMR (500 MHz, $\left.\mathrm{CD}_{3} \mathrm{OD}\right) \delta 8.19(\mathrm{~d}, J=1.5 \mathrm{~Hz}, 1 \mathrm{H}), 8.14$ (d, $J$ = 8.8 Hz, $1 \mathrm{H}), 8.08-8.12(\mathrm{~m}, 2 \mathrm{H}), 7.98(\mathrm{dd}, J=8.8,1.5 \mathrm{~Hz}, 1 \mathrm{H}), 7.82(\mathrm{~s}, 1 \mathrm{H}), 7.70-7.80(\mathrm{~m}, 4 \mathrm{H})$, 6.04 (brs, $1 \mathrm{H}$ ), 5.71-5.80 (m, $1 \mathrm{H}$ ), 5.75 (ddd, $J=17.1,10.3,8.8 \mathrm{~Hz}, 1 \mathrm{H}$ ), 5.30 (dd, $J=17.1,1.2 \mathrm{~Hz}, 1$ H), 5.13 (dd, $J=10.5,1.6 \mathrm{~Hz}, 1 \mathrm{H}), 4.58$ (dd, $J=10.7,6.8 \mathrm{~Hz}, 1 \mathrm{H}$ ), 4.41 (t, $J=7.6 \mathrm{~Hz}, 1 \mathrm{H}$ ), 4.22- 
4.30 (m, $1 \mathrm{H}$ ), 4.13 (dd, $J=12.0,2.9 \mathrm{~Hz}, 1 \mathrm{H}$ ), 3.75-3.85 (m, $1 \mathrm{H}$ ), 2.80-3.05 (m, $3 \mathrm{H}$ ), 2.69 (dd, $J=$ 13.9, $6.6 \mathrm{~Hz}, 1 \mathrm{H}), 2.40-2.48$ (m, $1 \mathrm{H}), 2.20$ (q, $J=8.8 \mathrm{~Hz}, 1 \mathrm{H}), 1.50-1.95$ (m, $6 \mathrm{H}), 1.15-1.42$ (m, 8 $\mathrm{H}), 1.16-1.14(\mathrm{~m}, 2 \mathrm{H}), 0.93-0.98$ (m, $3 \mathrm{H}) \mathrm{ppm}$; HRMS (ESI) $\mathrm{m} / \mathrm{z} 772.3378\left[(\mathrm{M}+\mathrm{H})^{+}\right.$; calcd for $\left.\mathrm{C}_{41} \mathrm{H}_{50} \mathrm{~N}_{5} \mathrm{O}_{8} \mathrm{~S}: 772.3375\right] ;[\alpha]_{D}^{25}-47.9^{\circ}(\mathrm{c}=0.26, \mathrm{MeOH})$.

Synthesis of (3R,5S,8S)-8-t-Butyl-N-((1R,2S)-1-\{[(cyclopropylsulfonyl)amino]carbonyl\}-2vinylcyclopropyl)-7,10-dioxo-22-phenyl-2,11-dioxa-6,9,21triazatetracyclo[15.6.2.1 3,6 .0 20,24 ]hexacosa-1(23),17,19,21,24-pentaene-5-carboxamide (16)

Step 1: Methyl 3-methyl-N-[(pent-4-en-1-yloxy)carbonyl]-L-valyl-(4R)-4-[(2-phenyl-6vinylquinolin-4-yl)oxy]-L-prolinate (16_1)

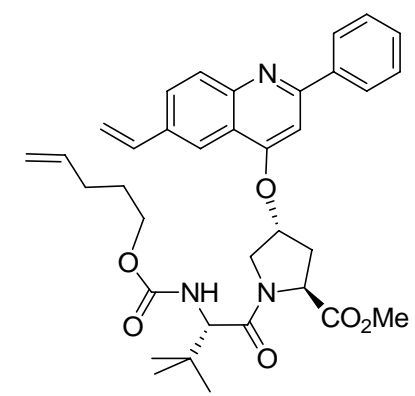

Prepared in the same manner as 9a (75\%). ${ }^{1} \mathrm{H}$ NMR (400 MHz, $\mathrm{CDCl}_{3}$ ) (mixture of Boc rotamers, major rotamer) $\delta 8.06(\mathrm{~m}, 3 \mathrm{H}), 7.98(\mathrm{~s}, 1 \mathrm{H}), 7.84-7.92(\mathrm{~m}, 1 \mathrm{H})$, 7.44-7.54 (m, $3 \mathrm{H}), 7.06(\mathrm{~s}, 1 \mathrm{H})$, (6.91 (dd, $J=17.6,10.9 \mathrm{~Hz}, 1 \mathrm{H}), 5.89$ (d, $J=17.6 \mathrm{~Hz}$ ), 5.70-5.82 (m, $1 \mathrm{H}), 5.2-5.42$ (m, $3 \mathrm{H}), 4.90-$ 5.04 (m, 2 H), 4.81 (t, $J=8.3 \mathrm{~Hz}, 1 \mathrm{H}), 4.44$ (d, $J=11.3 \mathrm{~Hz}, 1 \mathrm{H}), 4.34$ (d, $J=9.7 \mathrm{~Hz}, 1 \mathrm{H}), 3.80-4.18$ (m, $4 \mathrm{H}), 3.78$ (s, $3 \mathrm{H}), 2.74-2.84(\mathrm{~m}, 1 \mathrm{H}), 2.34-2.44(\mathrm{~m}, 1 \mathrm{H}), 2.02-2.10(\mathrm{~m}, 2 \mathrm{H}), 1.58-1.68(\mathrm{~m}, 2 \mathrm{H})$, 1.08 (s, 9 H) ppm; HRMS (ESI) $\mathrm{m} / \mathrm{z} 600.3083$ [(M+H) ${ }^{+}$; calcd for $\left.\mathrm{C}_{35} \mathrm{H}_{42} \mathrm{~N}_{3} \mathrm{O}_{6}: 600.3068\right]$.

Step 2: Methyl (3R,5S,8S,15E)-8-tert-butyl-7,10-dioxo-22-phenyl-2,11-dioxa-6,9,21triazatetracyclo[15.6.2.1 3,6 .0 20,24 ]hexacosa-1(23),15,17,19,21,24-hexaene-5-carboxylate (16_2)

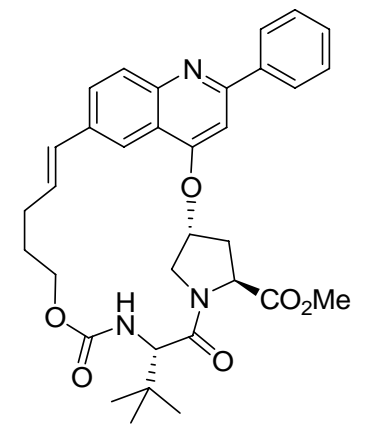

Prepared in the same manner as 10a (49\%). ${ }^{1} \mathrm{H}$ NMR $\left(400 \mathrm{MHz}, \mathrm{CDCl}_{3}\right) \delta 8.09(\mathrm{~s}, 1 \mathrm{H}), 8.03$ (d, $J=7.3$ $\mathrm{Hz}, 2 \mathrm{H}), 7.97$ (d, $J=8.3 \mathrm{~Hz}, 1 \mathrm{H}), 7.38-7.56(\mathrm{~m}, 4 \mathrm{H}), 7.05$ (s, $1 \mathrm{H}), 6.48$ (d, $J=15.7 \mathrm{~Hz}, 1 \mathrm{H}), 6.30$ (dt, $J=15.7,7.2 \mathrm{~Hz}, 1 \mathrm{H}), 5.51$ (d, $J=9.2 \mathrm{~Hz}, 1 \mathrm{H}), 5.30$ (brs, $1 \mathrm{H}), 4.71$ (t, $J=9.1 \mathrm{~Hz}, 1 \mathrm{H}), 4.59$ (d, $J=$ $11.5 \mathrm{~Hz}, 1 \mathrm{H}), 4.43-4.55$ (m, $2 \mathrm{H}), 3.86-3.96$ (m, $2 \mathrm{H}$ ), 3.72 (s, $3 \mathrm{H}), 2.85$ (dd, $J=13.8,7.7 \mathrm{~Hz}, 1 \mathrm{H}$ ), 2.32-2.42 (m, 2 H), 2.17-2.26 (m, $1 \mathrm{H}), 1.91-2.02(\mathrm{~m}, 1 \mathrm{H}), 1.72-1.83$ (m, $1 \mathrm{H}), 1.08$ (s, $9 \mathrm{H}) \mathrm{ppm}$; HRMS (ESI) $m / z$ 572.2758 [(M+H) ${ }^{+}$; calcd for $\left.\mathrm{C}_{33} \mathrm{H}_{38} \mathrm{~N}_{3} \mathrm{O}_{6}: 572.2755\right]$. 
Step 3: (3R,5S,8S)-8-t-Butyl-N-((1R,2S)-1-\{[(cyclopropylsulfonyl)amino]carbonyl\}-2-

vinylcyclopropyl)-7,10-dioxo-22-phenyl-2,11-dioxa-6,9,21-

triazatetracyclo[15.6.2.1 3,6 .0 20,24 ]hexacosa-1(23),17,19,21,24-pentaene-5-carboxamide (16)

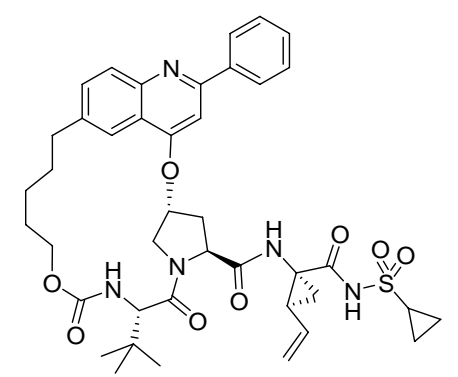

Prepared in the same manner as 15 (34\%). ${ }^{1} \mathrm{H}$ NMR $\left(400 \mathrm{MHz}, \mathrm{CD}_{3} \mathrm{OD}\right) \delta 9.14$ (s, $\left.1 \mathrm{H}\right), 8.12$ (d, $J=8.7$ Hz, $1 \mathrm{H}$ ), 8.03-8.09 (m, $3 \mathrm{H}$ ), 7.96 (dd, $J=8.8,1.8 \mathrm{~Hz}, 1 \mathrm{H}$ ), 7.82 (s, $1 \mathrm{H}), 7.68-7.78$ (m, $3 \mathrm{H}$ ), 6.02 (brs, $1 \mathrm{H}$ ), 5.70 (ddd, $J=19.1,10.3,9.0 \mathrm{~Hz}, 1 \mathrm{H}$ ), 5.25 (dd, $J=17.2,1.2 \mathrm{~Hz}, 1 \mathrm{H}$ ), 5.10 (dd, $J=10.3,1.5 \mathrm{~Hz}$, 1 H), 4.56-4.64 (m, 2 H), 4.23-4.32 (m, 2 H), 4.07 (dd, $J=12.3,2.8 \mathrm{~Hz}, 1$ H), 3.73 (ddd, $J=11.1$, 6.6, $4.5 \mathrm{~Hz}, 1 \mathrm{H}$ ), 3.02 (dt, $J=13.6,6.3 \mathrm{~Hz}, 1 \mathrm{H}$ ), 2.92 (ddd, $J=12.8,8.1,4.8 \mathrm{~Hz}, 1 \mathrm{H}$ ), 2.64-2.81 (m, $2 \mathrm{H}$ ), 2.38 (ddd, $J=14.2,10.6,3.6 \mathrm{~Hz}, 1 \mathrm{H}$ ), 2.18 (q, $J=8.8 \mathrm{~Hz}, 1 \mathrm{H}), 1.66-1.88$ (m, $4 \mathrm{H}), 1.42-1.53$ (m, 1 ), 1.40 (dd, $J=9.5,5.5 \mathrm{~Hz}, 1 \mathrm{H}$ ), 1.18-1.28 (m, $4 \mathrm{H}$ ), 1.0-1.10 (m, $1 \mathrm{H}$ ), 1.04 (s, $9 \mathrm{H}$ ) ppm; HRMS (ESI) $\mathrm{m} / \mathrm{z} 772.3345\left[(\mathrm{M}+\mathrm{H})^{+}\right.$; calcd for $\left.\mathrm{C}_{41} \mathrm{H}_{50} \mathrm{~N}_{5} \mathrm{O}_{8} \mathrm{~S}: 772.3375\right]$; $[\alpha]_{D}^{25}-51.0^{\circ}$ (c = 0.35, MeOH).

Ethyl (4R)-4-[(7-bromoisoquinolin-1-yl)oxy]-L-prolinate hydrochloride (19)<smiles>CCOC1CC(Oc2nccc3ccc(Br)cc23)N1</smiles>

To a solution of trans 4-hydroxy L-BOC-proline (50 g, $0.216 \mathrm{~mol})$ in DMSO (100 mL) at RT was added potassium t-butoxide ( $73 \mathrm{~g}, 0.65 \mathrm{~mol}$ ) in a single portion. The mixture was stirred at RT for $30 \mathrm{~min}$, cooled to $17^{\circ} \mathrm{C}$ and 7-bromo-1-chloroisoquinoline ( $52.4 \mathrm{~g}, 0.216 \mathrm{~mol}$ ) added in 2 portions. The reaction was allowed to warm to RT, stirred for $18 \mathrm{~h}$., quenched into ice-cold $10 \%$ citric acid solution (4 $\mathrm{L}$ ) and extracted with EtOAc (2L). The organic layer was washed with brine (2L) and the aqueous phases back extracted with EtOAc (1L). The combined organic phases were dried over anhydrous $\mathrm{Na}_{2} \mathrm{SO}_{4}$ and the solvent evaporated to give a dark solid (88.8 gm) The solid was dissolved in ethanol $\left(2.0 \mathrm{~L}\right.$ ) cooled to $10^{\circ} \mathrm{C}$ and $\mathrm{HCl}$ bubbled through until the solution was saturated, maintaining the internal temperature $<45^{\circ} \mathrm{C}$. The reaction mixture was then stirred at RT for $24 \mathrm{~h}$. The mixture was then concentrated under reduced pressure to $1 / 2$ volume and diluted with hexane. The mixture was filtered to give $57.0 \mathrm{~g}(65 \%)$ of $\mathbf{1 9}$ as a gray solid used directly in the next step. ${ }^{1} \mathrm{H}$ NMR (400 MHz, $\left.\mathrm{CD}_{3} \mathrm{OD}\right) \delta 8.49-8.51(\mathrm{~m}, 1 \mathrm{H}), 8.03(\mathrm{~d}, J=5.9 \mathrm{~Hz}, 1 \mathrm{H}), 7.86$ (dd, $\left.J=8.7,2.0 \mathrm{~Hz}, 1 \mathrm{H}\right), 7.80$ (d, $J=8.70$ $\mathrm{Hz}, 1 \mathrm{H}$ ), 7.41 (dd, $J=5.8,0.7 \mathrm{~Hz}, 1 \mathrm{H}$ ), 5.97 (t, $J=4.5 \mathrm{~Hz}, 1 \mathrm{H}$ ), 4.36 (dq, $J=0.8,7.1 \mathrm{~Hz}, 1 \mathrm{H}$ ), 3.87 (dd, $J=13.1,4.4 \mathrm{~Hz}, 1 \mathrm{H}$ ), 3.75-3.83 (m, $1 \mathrm{H}$ ), 2.90 (tq, $J=1.6,7.8 \mathrm{~Hz}, 1 \mathrm{H}$ ), 2.61 (ddd, $J=15.3,10.3$, $5.0 \mathrm{~Hz}, 1 \mathrm{H}$ ), 1.35 (t, $J=7.1 \mathrm{~Hz}, 3 \mathrm{H}$ ) ppm; HRMS (ESI) $\mathrm{m} / \mathrm{z} 365.0489\left[(\mathrm{M}+\mathrm{H})^{+}\right.$; calcd for $\mathrm{C}_{16} \mathrm{H}_{18} \mathrm{BrN}_{2} \mathrm{O}_{3}$ : 365.0495]. 


\section{3-Methyl-N-[(pent-4-en-1-yloxy)carbonyl]-L-valine (20)}

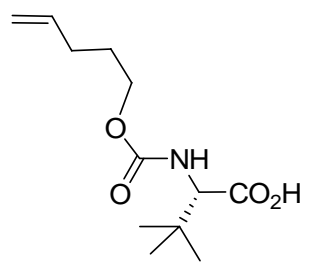

Diisopropylethylamine (9.85 g, $76.2 \mathrm{mmol}$ ) was added dropwise to a $0{ }^{\circ} \mathrm{C}$ solution of 4-penten-1-ol (7.22 g, $83.9 \mathrm{mmol})$ and triphosgene $(11.3 \mathrm{~g}, 38.1 \mathrm{mmol})$ in dioxane $(160 \mathrm{~mL})$. The resulting white suspension was stirred for $5 \mathrm{~min}$ at $0{ }^{\circ} \mathrm{C}$, then allowed to warm to RT over $1 \mathrm{~h}$. The suspension was recooled to $0{ }^{\circ} \mathrm{C}$ and $1 \mathrm{~N} \mathrm{NaOH}(76.2 \mathrm{~mL})$ and L-tert-butylglycine $(10.0 \mathrm{~g}, 76.2 \mathrm{mmol})$ added. The reaction mixture was allowed to warm to $\mathrm{RT}$ and stirred for $18 \mathrm{~h}$. The dioxane was removed in vacuo, the reaction mixture basified to $\mathrm{pH} 12$ with $1 \mathrm{~N} \mathrm{NaOH}$ and extracted with DCM (3x $150 \mathrm{~mL})$ and the organic phases discarded. The aquesous phase was acidified to $\sim \mathrm{pH} 1$ with $6 \mathrm{~N}$ HCland extracted with DCM (3 x $150 \mathrm{~mL}$ ). The combined organic layers were dried over MgSO4 and concentrated to give 20 as a tan oil (13.7 g, 73.9\% yield). ${ }^{1} \mathrm{H}$ NMR (400 MHz, $\left.\mathrm{CD}_{3} \mathrm{OD}\right) \delta$ 5.77-5.89 (m, $\left.1 \mathrm{H}\right), 5.03$ (dq, $J=$ 17.1, 1.7 Hz, 1 H), 4.87 (brs, 1 H), 4.05 (t, $J=6.6$ Hz, 2 H), 3.99 (s, 1 H), 2.1-2.2 (m, 2 H), 1.68-1.77 (m, $2 \mathrm{H}) 4.96$ (brd, $J=10.3 \mathrm{~Hz}, 1 \mathrm{H}$ ) ppm; HRMS (ESI) $\mathrm{m} / \mathrm{z} 244.1541\left[(\mathrm{M}+\mathrm{H})^{+}\right.$; calcd for $\mathrm{C}_{12} \mathrm{H}_{22} \mathrm{NO}_{4}$ : 244.1543].

Ethyl 3-methyl-N-[(pent-4-en-1-yloxy)carbonyl]-L-valyl-(4R)-4-[(7-bromoisoquinolin-1-yl)oxy]-Lprolinate (21)

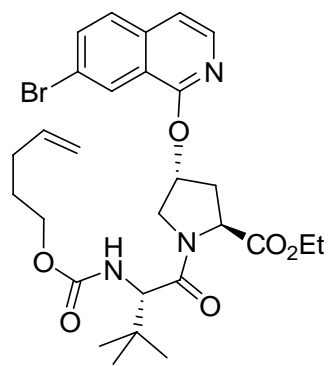

To a solution of 19 (20.34 g, $0.0509 \mathrm{~mol})$ in DMF (160 mL) was added 20 (11.8 g, $0.0484 \mathrm{~mol})$, DIPEA (35.5 mL, $0.203 \mathrm{~mol}$ ) and TBTU (24.5 g, $0.076 \mathrm{~mol}$ ) and the reaction mixture stirred at RT for $18 \mathrm{~h}$. The mixture was partitioned between $10 \%$ aqueous citric acid/water $(1 \mathrm{~L})$ and EtOAc $(1 \mathrm{~L})$, the organic layer washed with water $(1 \mathrm{~L})$ and then saturated $\mathrm{NaHCO}_{3}(1 \mathrm{~L})$. The aqueous extracts were reextracted with EtOAc and the combined organic extracts dried over anhydrous $\mathrm{Na}_{2} \mathrm{SO}_{4}$. The solvent was then evaporated and the crude product purified by chromatography on silica (20-60\% EtOAc hexane) to give 21 (22.7 g, 80\%). ${ }^{1} \mathrm{H}$ NMR (400 MHz, $\mathrm{CD}_{3} \mathrm{OD}$ ) (mixture of Boc rotamers, major rotamer) $\delta 8.24$ (brs, $1 \mathrm{H}), 7.94(\mathrm{~d}, J=5.9 \mathrm{~Hz}, 1 \mathrm{H}), 7.72(\mathrm{dd}, J=8.8,1.7 \mathrm{~Hz}, 1 \mathrm{H}), 7.65(\mathrm{~d}, J=8.7 \mathrm{~Hz}, 1$ H), 7.25 (d, $J=5.9 \mathrm{~Hz}, 1 \mathrm{H}), 5.64-5.83(\mathrm{~m}, 2 \mathrm{H}), 4.85-4.95(\mathrm{~m}, 2 \mathrm{H}), 4.67$ (t, $J=8.7 \mathrm{~Hz}, 1 \mathrm{H}), 4.43$ (d, $J$ $=11.8 \mathrm{~Hz}, 1 \mathrm{H}), 4.22(\mathrm{~s}, 1 \mathrm{H}), 4.17$ (q, J = 7.0 Hz, $1 \mathrm{H}), 3.94-4.04(\mathrm{~m}, 1 \mathrm{H}), 3.66-3.82(\mathrm{~m}, 2 \mathrm{H}), 2.66-$ 2.74 (m, $1 \mathrm{H}$ ), 2.32 (ddd, $J=14.0,9.4,4.6 \mathrm{~Hz}, 1 \mathrm{H}$ ), 1.96 (q, $J=7.2 \mathrm{~Hz}, 1 \mathrm{H}$ ), 1.49 (quin, $J=7.1 \mathrm{~Hz}, 1$ $\mathrm{H}), 1.24$ (t, $J=7.1 \mathrm{~Hz}, 3 \mathrm{H}), 1.03$ (s, $9 \mathrm{H}$ ) ppm; HRMS (ESI) $\mathrm{m} / \mathrm{z} 590.1866$ [(M+H) ${ }^{+}$; calcd for $\mathrm{C}_{28} \mathrm{H}_{37} \mathrm{~N}_{3} \mathrm{O}_{6}$ : 590.1860]. 
Ethyl 3-methyl-N-[(pent-4-en-1-yloxy)carbonyl]-L-valyl-(4R)-4-[(7-vinylisoquinolin-1-yl)oxy]-Lprolinate (22)

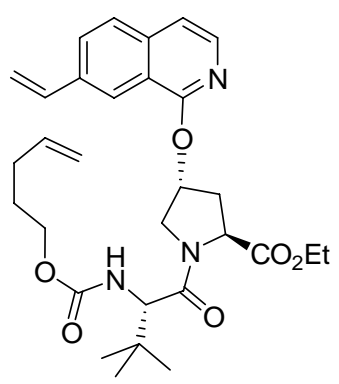

Bromide 21 (74 g, $126 \mathrm{mmol})$ was dissolved in toluene (1500 mL) and nitrogen bubbled through for 30 min. Tributylvinyltin (45.7 mL, $156 \mathrm{mmol}$ ) and tetrakis(triphenylphosphine)palladium(0) (14.5 g, 12.5 mmol) were added and the reaction mixture heated to $100^{\circ} \mathrm{C}$ under nitrogen. After $3 \mathrm{~h}$, the reaction was complete, the volatiles were evaporated and the residue purified by silica gel chromatography (10-50\% EtOAc / hexane) to give 22 as a clear oil (59.5 g, 87\%). ${ }^{1} \mathrm{H}$ NMR (400 MHz, CD ${ }_{3} \mathrm{OD}$ ) (mixture of Boc rotamers, major rotamer) $\delta 8.10(\mathrm{~s}, 1 \mathrm{H}), 7.90-7.95(\mathrm{~m}, 2 \mathrm{H}), 7.78(\mathrm{~d}, J=8.5 \mathrm{~Hz}, 1 \mathrm{H}), 7.32(\mathrm{~d}, J=5.9$ Hz, 1 H), 6.92 (dd, $J=17.7,11.0 \mathrm{~Hz}, 1 \mathrm{H}$ ), 5.93 (d, $J=17.6 \mathrm{~Hz}, 1 \mathrm{H}$ ), 5.85 (brs, $1 \mathrm{H}$ ), 5.67-5.80 (m, 1 H), 5.35 (d, $J=10.9 \mathrm{~Hz}, 1 \mathrm{H}), 4.88-4.98(\mathrm{~m}, 1 \mathrm{H}), 4.73$ (t, $J=8.6 \mathrm{~Hz}, 1 \mathrm{H}), 4.47$ (d, $J=11.9 \mathrm{~Hz}, 1 \mathrm{H})$, 4.28 (s, $1 \mathrm{H}), 4.21$ (q, $J=7.1 \mathrm{~Hz}, 2 \mathrm{H}), 4.06$ (dd, $J=12.0,3.5 \mathrm{~Hz}, 1 \mathrm{H}), 3.77$ (dt, $J=10.4,6.8 \mathrm{~Hz}, 1 \mathrm{H})$, 3.64 (dt, $J=10.4,6.7 \mathrm{~Hz}, 1 \mathrm{H}$ ), 2.77 (dd, $J=13.9,8.0 \mathrm{~Hz}, 1 \mathrm{H}$ ), 2.38 (ddd, $J=13.9,9.4,4.6 \mathrm{~Hz}, 1 \mathrm{H}$ ), 1.99 (q, $J=7.1 \mathrm{~Hz}, 1 \mathrm{H}), 1.29$ (t, $J=7.1 \mathrm{~Hz}, 1 \mathrm{H}), 1.06$ (s, $9 \mathrm{H}$ ) ppm; HRMS (ESI) m/z 538.2894 $\left[(\mathrm{M}+\mathrm{H})^{+}\right.$; calcd for $\mathrm{C}_{30} \mathrm{H}_{40} \mathrm{~N}_{3} \mathrm{O}_{6}$ : 538.2912].

Ethyl (3R,5S,8S,15E)-8-tert-butyl-7,10-dioxo-2,11-dioxa-6,9,23triazatetracyclo[15.6.2.1 3,6 .0 20,24 ]hexacosa-1(23),15,17,19,21,24-hexaene-5-carboxylate (23a)<smiles>CCOC(=O)[C@@H]1C[C@@H]2CN1C(=O)[C@H](C(C)(C)C)NC(=O)OCCC/C=C/c1ccc3ccnc(c3c1)O2</smiles>

The olefin 22 (58 g, $108 \mathrm{mmol}$ ) was dissolved in DCM (11.6 L) and nitrogen bubbled through the solution for $30 \mathrm{~min}$. Bis(tricyclohexylphosphine)-3-phenyl-1H-indene-1ylideneruthenium dichloride (Neolyst M1 catalyst, Strem Chemicals, CAS\#250220-36-1) (17 gm, 18 mmol) dissolved in degassed dichloromethane $(4.6 \mathrm{~L})$ was added over $4 \mathrm{hr}$ and the reaction stirred at RT. After $5 \mathrm{~h}$, additional catalyst (6 g) in DCM was added and the reaction stirred for $18 \mathrm{~h}$. The mixture was concentrated to $1 \mathrm{~L}$, acetone $(150 \mathrm{~mL})$ added and the mixture passed through a pad of silica and the silica pad washed with $15 \%$ acetone/DCM. The combined filtrates were concentrated and the residue purified by chromatography on silica gel (DCM load) (20-60\% EtOAc / hexane) to give 23a (46.4 g, 83\%) together with some mixed cis trans material (6.7 g). $\left.{ }^{1} \mathrm{H} \mathrm{NMR} \mathrm{(400} \mathrm{MHz,} \mathrm{CD} \mathrm{CD}_{3}\right) \delta 8.37$ (brs, $\left.1 \mathrm{H}\right), 7.88$ (d, $J=$ 
$5.9 \mathrm{~Hz}, 1 \mathrm{H}), 7.73$ (d, $J=8.3 \mathrm{~Hz}, 1 \mathrm{H}), 7.58$ (dd, $J=8.4,1.7 \mathrm{~Hz}, 1 \mathrm{H}), 5.30$ (dd, $J=6.0,0.6 \mathrm{~Hz}, 1 \mathrm{H}$ ), $6.57(\mathrm{~d}, J=15.8 \mathrm{~Hz}, 1 \mathrm{H}), 6.40$ (dt, $J=15.8,7.3 \mathrm{~Hz}, 1 \mathrm{H}) 5.79(\mathrm{t}, J=3.20 \mathrm{~Hz}, 1 \mathrm{H}), 4.43-4.63(\mathrm{~m}, 4 \mathrm{H})$, 4.12-4.24 (m, 2 H), 3.92-3.99 (m, 2 H), 2.79 (dq, $J=1.6,7.8$ Hz, 1 H), 2.30-2.42 (m, 2 H), 2.26 (ddd, $J$ = 14.2, 10.6, $3.8 \mathrm{~Hz}, 1 \mathrm{H}), 1.76-2.03(\mathrm{~m}, 2 \mathrm{H}), 1.25$ (t, $J=7.1 \mathrm{~Hz}, 3 \mathrm{H}), 1.12$ (s, $9 \mathrm{H})$ ppm; HRMS (ESI) $\mathrm{m} / \mathrm{z} 510.2596\left[(\mathrm{M}+\mathrm{H})^{+}\right.$; calcd for $\mathrm{C}_{28} \mathrm{H}_{36} \mathrm{~N}_{3} \mathrm{O}_{6}$ : 510.2599].

Ethyl (3R,5S,8S)-8-tert-butyl-7,10-dioxo-2,11-dioxa-6,9,23triazatetracyclo[15.6.2.1 3,6 .0 20,24 ]hexacosa-1(23),17,19,21,24-pentaene-5-carboxylate (23b)<smiles>CCOC(=O)[C@@H]1C[C@@H]2CN1C(=O)[C@H](C(C)(C)C)NC(=O)OCCCCCc1ccc3ccnc(c3c1)O2</smiles>

A solution of 23a (40 mg, $0.078 \mathrm{mmol})$ in EtOH (10 mL) was treated with 10\% Pd/C (8 mg) and hydrogenated under a balloon of hydrogen for $24 \mathrm{~h}$. The reaction mixture was filtered and the filtrate concentrated to afford 23b (40 mg, 100\%). ${ }^{1} \mathrm{H}$ NMR (400 MHz, CD $\left.{ }_{3} \mathrm{OD}\right) \delta 7.89$ (brs, $\left.1 \mathrm{H}\right), 7.84$ (d, $J=$ $6.0 \mathrm{~Hz}, 1 \mathrm{H}), 7.70$ (d, $J=8.3 \mathrm{~Hz}, 1 \mathrm{H}), 7.54$ (dd, $J=8.3,1.7 \mathrm{~Hz}, 1 \mathrm{H}), 7.28$ (d, $J=6.0 \mathrm{~Hz}, 1 \mathrm{H}), 6.10$ (t, $J=3.3 \mathrm{~Hz}, 1 \mathrm{H}$ ), 4.67 (dd, $J=10.4,7.8 \mathrm{~Hz}, 1 \mathrm{H}$ ), 4.43 (dd, $J=11.7,1.7 \mathrm{~Hz}, 1 \mathrm{H}), 4.27-4.37$ (m, $2 \mathrm{H}$ ), 4.16 (dq, $J=1.0,7.1 \mathrm{~Hz}, 2 \mathrm{H}$ ), 3.90 (dd, $J=11.8,3.0 \mathrm{~Hz}, 1 \mathrm{H}$ ), 3.69 (ddd, $J=11.0,6.5,4.4 \mathrm{~Hz}, 1 \mathrm{H}$ ), 2.85 (dt, $J=13.6,6.0$ Hz, 1 H), 2.59-2.70 (m, 2 H), 2.26 (ddd, $J=14.1,10.4$, 3.9 Hz, 1 H), 1.60-1.76 (m, $3 \mathrm{H}), 1.37-1.48$ (m, $1 \mathrm{H}), 1.23$ (t, $J=7.1 \mathrm{~Hz}, 3 \mathrm{H}), 1.14-1.24$ (m, $2 \mathrm{H}), 1.03$ (s, $9 \mathrm{H}) \mathrm{ppm}$; HRMS (ESI) $\mathrm{m} / \mathrm{z} 512.2743\left[(\mathrm{M}+\mathrm{H})^{+}\right.$; calcd for $\mathrm{C}_{28} \mathrm{H}_{38} \mathrm{~N}_{3} \mathrm{O}_{6}$ : 512.2755].

\section{(1R,2S)-1-amino-N-(cyclopropylsulfonyl)-2-vinylcyclopropanecarboxamide (24)}<smiles>C=CCC(N)C(=O)NS(=O)(=O)C1CC1</smiles>

24 was prepared as described previously (Wang, X. A.; Sun, L.-Q.; Sit, S.-Y.; Sin, Y.; Scola, P. M.; Hewawasam, P.; Good, A. C.; Chen, Y.; Campbell, J. A. US 6,995,174, 2006). 
(3R,5S,8S,15E)-8-t-Butyl-N-((1R,2S)-1-\{[(cyclopropylsulfonyl)amino]carbonyl\}-2vinylcyclopropyl)-7,10-dioxo-2,11-dioxa-6,9,23-triazatetracyclo[15.6.2.1 3,6 .0 20,24 ]hexacosa1(23),15,17,19,21,24-hexaene-5-carboxamide (25a)<smiles>C=CCC(NC(=O)C1C[C@H]2CC1N(C(=O)[C@H](NC(=O)OCCC/C=C/c1ccc3ccnc(-c4ccncc4)c3c1)C(C)(C)C)C2)C(=O)NS(=O)(=O)C1CC1</smiles>

A solution of 23a (11.33 g, $22.3 \mathrm{mmol})$ in THF $(300 \mathrm{~mL})$ and EtOH $(150 \mathrm{~mL})$ was treated with aqueous $\mathrm{LiOH}(4.0 \mathrm{~g}$ in $150 \mathrm{~mL})$. The reaction mixture was stirred at $40^{\circ} \mathrm{C}$ for $1.5 \mathrm{~h}$, after which $3 \mathrm{M} \mathrm{HCl}(58$ $\mathrm{mL}$ ) was added and the mixture evaporated. The solid residue was partitioned between EtOAc (700 $\mathrm{mL})$ and water $(400 \mathrm{~mL})$ and the organic layer separated, dried over anhydrous $\mathrm{Na}_{2} \mathrm{SO}_{4}$, filtered and concentrated to a foam. The foam was dissolved in DCM $(900 \mathrm{~mL})$ and $\mathbf{2 4}$ (hydrochloride salt, $7.51 \mathrm{gm}$, $28 \mathrm{mmol}$ ), DIPEA (13.6 mL, $78 \mathrm{mmol})$, DMAP (1.5 gm, $12 \mathrm{mmol}$ ) and HATU ( $12.0 \mathrm{gm}, 31.5 \mathrm{mmol}$ ) added and the reaction mixture stirred at RT for $18 \mathrm{~h}$. The mixture was concentrated to an oil, dissolved in EtOAc $(750 \mathrm{~mL})$ and water $(700 \mathrm{~mL})$ and the $\mathrm{pH}$ adjusted to 1.5 with 12M HCL. This mixture was stirred for $30 \mathrm{~min}$, the organic layer separated and aqueous layer back extracted with EtOAc. The organic extracts were washed with brine, dried over anhydrous $\mathrm{Na}_{2} \mathrm{SO}_{4}$ and treated with decolorizing carbon (1 g), filtered and concentrated to a foam, purified by chromatography on silica (40-60\% EtOAc/hexane) to afford 25a as a foam (13.0 g). The foam was dissolved in warm isopropanol (200 $\mathrm{mL}$ ), filtered and concentrated to $50 \mathrm{~mL}$. Crystallization of the product occurred upon slow cooling and the resulting thick slurry was diluted slowly with hexane $(150 \mathrm{~mL})$ and stirred for $18 \mathrm{~h}$. The solids were filtered washed with $20 \%$ isopropanol/hexanes and dried in vacuo $\left(50{ }^{\circ} \mathrm{C}\right)$ to give the potassium salt of 25a (12.2 g , 93\% recovery) as a white solid. ${ }^{1} \mathrm{H}$ NMR $\left(400 \mathrm{MHz}, \mathrm{CDCl}_{3}\right) \delta 9.89(\mathrm{~s}, 1 \mathrm{H}), 8.23(\mathrm{~s}, 1 \mathrm{H})$, 7.87 (d, $J=5.9 \mathrm{~Hz}, 1 \mathrm{H}$ ), 7.62 (d, $J=8.4 \mathrm{~Hz}, 1 \mathrm{H}$ ), 7.47 (dd, $J=1.6,8.3 \mathrm{~Hz}, 1 \mathrm{H}$ ), 7.17 (d, $J=5.9 \mathrm{~Hz}, 1$ H), 7.06 (s, $1 \mathrm{H}), 6.50$ (d, $J=15.8 \mathrm{~Hz}, 1 \mathrm{H}), 6.29-6.39$ (m, $1 \mathrm{H}), 5.81$ (brs, $1 \mathrm{H}), 5.65-5.76$ (m, $1 \mathrm{H}$ ), 5.61 (d, $J=9.3 \mathrm{~Hz}, 1 \mathrm{H}), 5.19$ (d, $J=17 \mathrm{~Hz}, 1 \mathrm{H}), 5.11$ (d, $J=10.3 \mathrm{~Hz}, 1 \mathrm{H}), 4.58$ (d, $J=10.9 \mathrm{~Hz}, 1 \mathrm{H})$, 4.40-4.55 (m, 3 H), 3.94-4.02 (m, 1 H), 3.90 (dd, $J=2.9,11.4$ Hz, 1 H), 2.83-2.92 (m, 1 H), 2.70-2.77 (m, $1 \mathrm{H}), 2.32-2.52(\mathrm{~m}, 3 \mathrm{H}), 1.6-2.05(\mathrm{~m}, 6 \mathrm{H}), 1.42$ (dd, $J=5.7,9.3 \mathrm{~Hz}, 1 \mathrm{H}), 1.22-1.34(\mathrm{~m}, 1 \mathrm{H})$, 0.94-1.12 (m, $10 \mathrm{H})$ ppm; HRMS (ESI) $\mathrm{m} / \mathrm{z} 694.2913$ [(M+H) ${ }^{+}$; calcd for $\left.\mathrm{C}_{35} \mathrm{H}_{44} \mathrm{~N}_{5} \mathrm{O}_{8} \mathrm{~S}: 694.2905\right]$; $[\alpha]_{D}$ ${ }^{25}+20.7^{\circ}$ (c = 0.52, MeOH); CHN found C : $58.16 \mathrm{H}: 6.28 \mathrm{~N}: 9.59$ Calc. For $\mathrm{C}_{35} \mathrm{H}_{43} \mathrm{~N}_{5} \mathrm{O}_{8} \mathrm{~S} .1 .5 \mathrm{H}_{2} \mathrm{O} \mathrm{C}$ : $58.32 \mathrm{H}: 6.43 \mathrm{~N}: 9.72$.

(3R,5S,8S)-8-t-Butyl-N-((1R,2S)-1-\{[(cyclopropylsulfonyl)amino]carbonyl\}-2-vinylcyclopropyl)7,10-dioxo-2,11-dioxa-6,9,23-triazatetracyclo[15.6.2.1 3,6 .0 20,24 ]hexacosa-1(23),17,19,21,24pentaene-5-carboxamide (25b) 


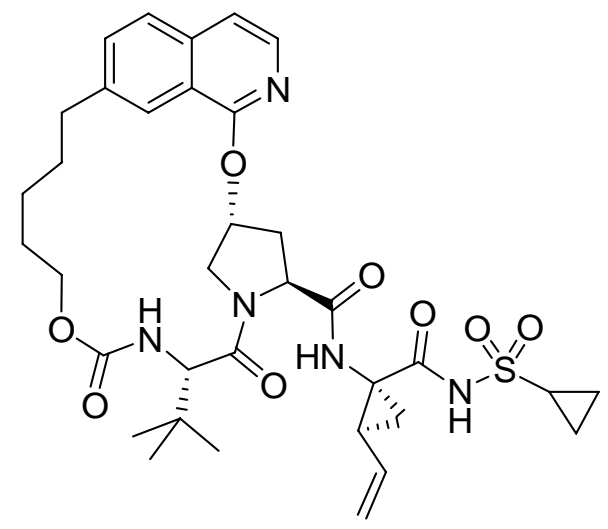

Prepared in the same manner as 25a. ${ }^{1} \mathrm{H}$ NMR (400 MHz, DMSO) (mixture of conformers, major conformer) $\delta 8.28(\mathrm{~s}, 1 \mathrm{H}), 8.01(\mathrm{~s}, 1 \mathrm{H}), 7.95(\mathrm{~d}, J=6.0 \mathrm{~Hz}, 1 \mathrm{H}), 7.80$ (d, $J=8.4 \mathrm{~Hz}, 1 \mathrm{H}), 7.62$ (dd, $J$ $=8.4,1.3 \mathrm{~Hz}, 1 \mathrm{H}), 7.34(\mathrm{~d}, J=5.9 \mathrm{~Hz}, 1 \mathrm{H}), 7.19(\mathrm{~d}, J=8.3 \mathrm{~Hz}, 1 \mathrm{H}), 6.58(\mathrm{~d}, J=15.8 \mathrm{~Hz}, 1 \mathrm{H}), 6.35$ (dt, $J=15.8,7.6 \mathrm{~Hz}, 1 \mathrm{H}$ ), 5.99 (dt, $J=19.0,7.6 \mathrm{~Hz}, 1 \mathrm{H}$ ), 5.64 (brs, $1 \mathrm{H}$ ), 5.02 (dd, $J=17.4,1.9 \mathrm{~Hz}, 1$ H), 4.84 (dd, $J=10.3,2.0 \mathrm{~Hz}, 1 \mathrm{H}), 4.25-4.43(\mathrm{~m}, 4 \mathrm{H}), 3.80-3.94$ (m, $2 \mathrm{H}), 3.44$ (q, $J=7.0 \mathrm{~Hz}, 1 \mathrm{H}$ ), 2.64-2.74 (m, 1 H), 2.50-2.58 (m, 1 H), 2.22-2.38 (m, $3 \mathrm{H}), 1.70-1.96$ (m, $3 \mathrm{H}), 1.49$ (dd, $J=7.2,4.1$ Hz, $1 \mathrm{H}$ ), 1.12 (m, $1 \mathrm{H}), 1.02$ (s, $9 \mathrm{H}), 0.70-0.80$ (m, $2 \mathrm{H}$ ), 0.56-0.66 (m, $2 \mathrm{H}$ ) ppm; HRMS (ESI) m/z $696.3066\left[(\mathrm{M}+\mathrm{H})^{+}\right.$; calcd for $\mathrm{C}_{35} \mathrm{H}_{46} \mathrm{~N}_{5} \mathrm{O}_{8} \mathrm{~S}$ : 696.3062]; $[\alpha]_{D}^{25}-0.57^{\circ}$ (c = 0.53, MeOH). 


\section{Compound Characterization data}

In general, $1 \mathrm{H}$ NMR data is provided except in cases where mixtures of rotamers/conformers are observed, in which case HPLC data under two different sets of conditions is provided (methods described in general experimental information) 
NMR and HPLC Data for Compound 6

${ }^{1} \mathrm{H}$ NMR Data $\left(400 \mathrm{MHz}, \mathrm{CDCl}_{3}\right.$ )

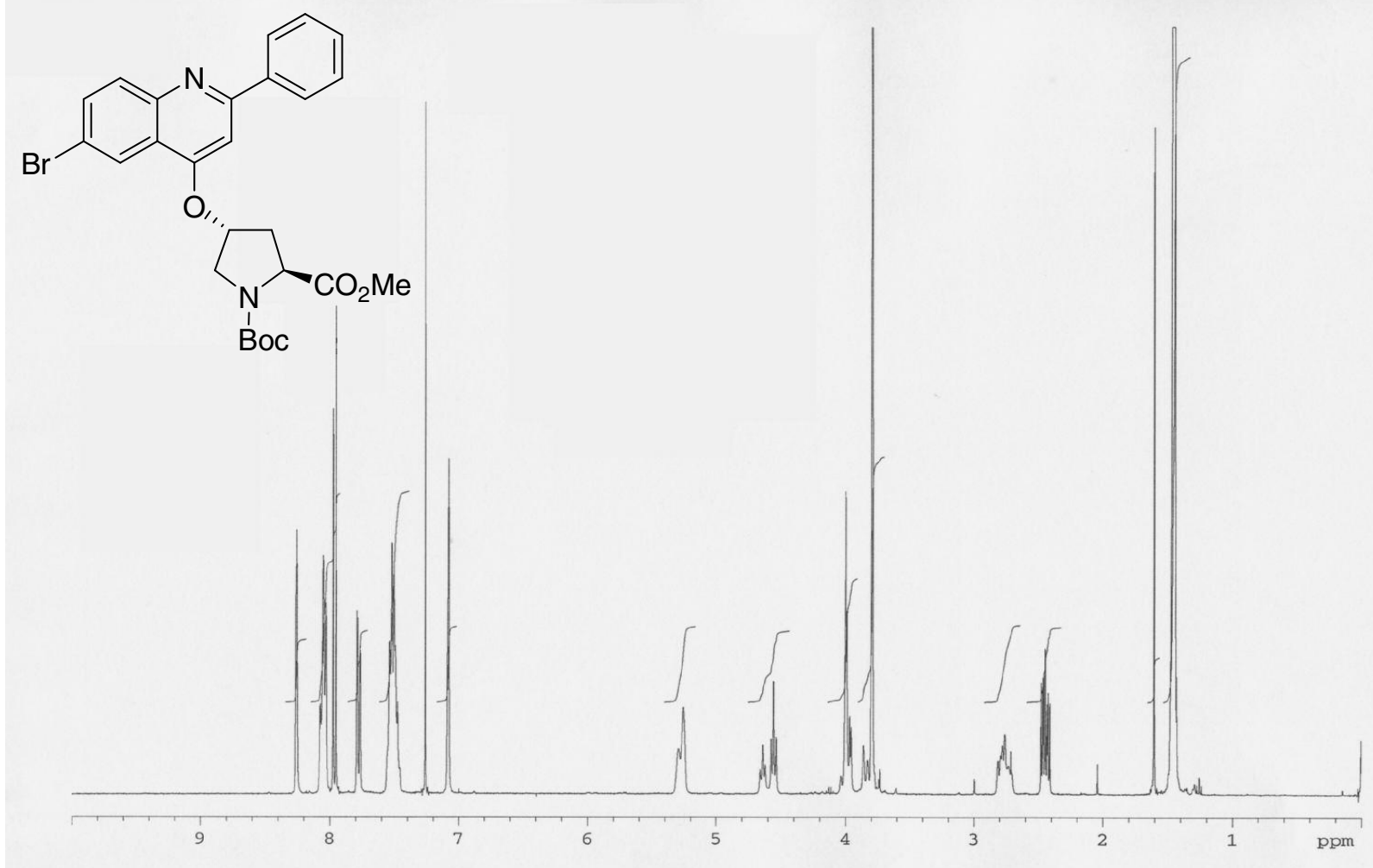

Method A HPLC Data

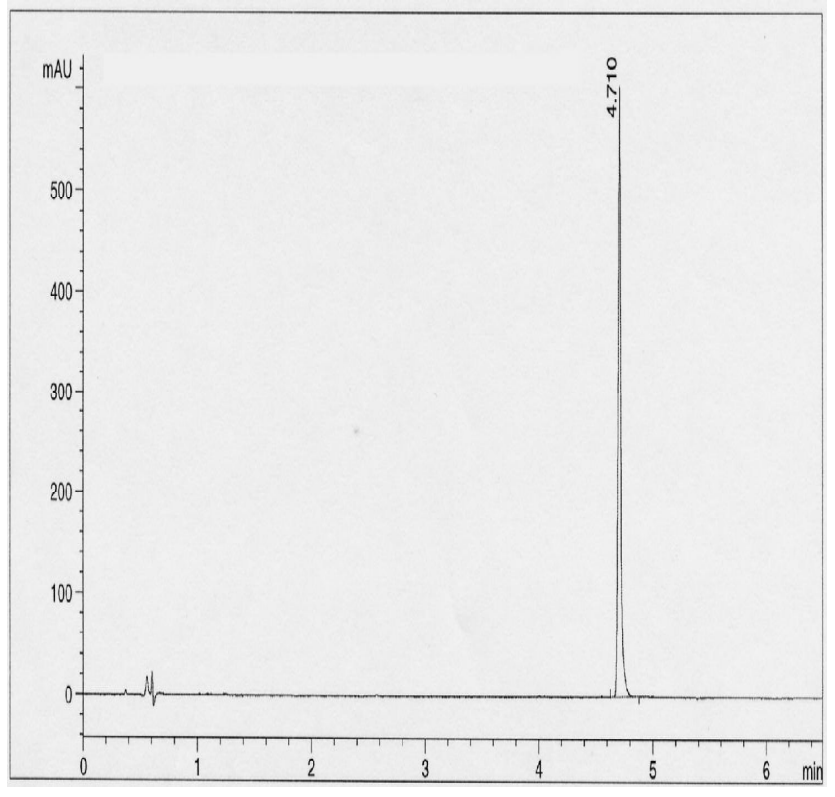

Method B HPLC Data

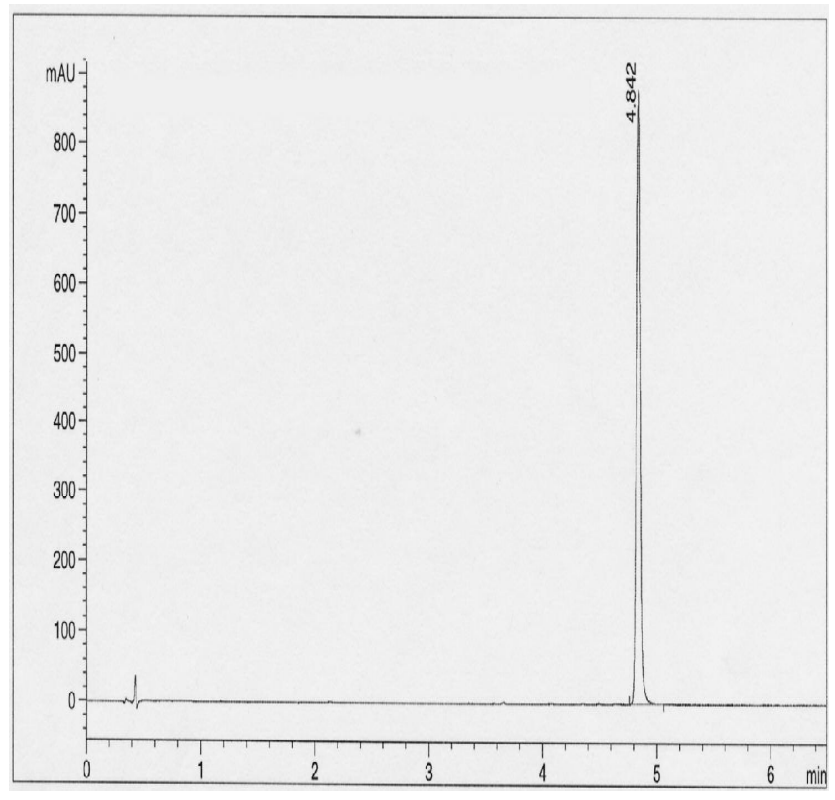


NMR and HPLC Data for Compound 7

${ }^{1} \mathrm{H}$ NMR Data $\left(400 \mathrm{MHz}, \mathrm{CDCl}_{3}\right.$ )

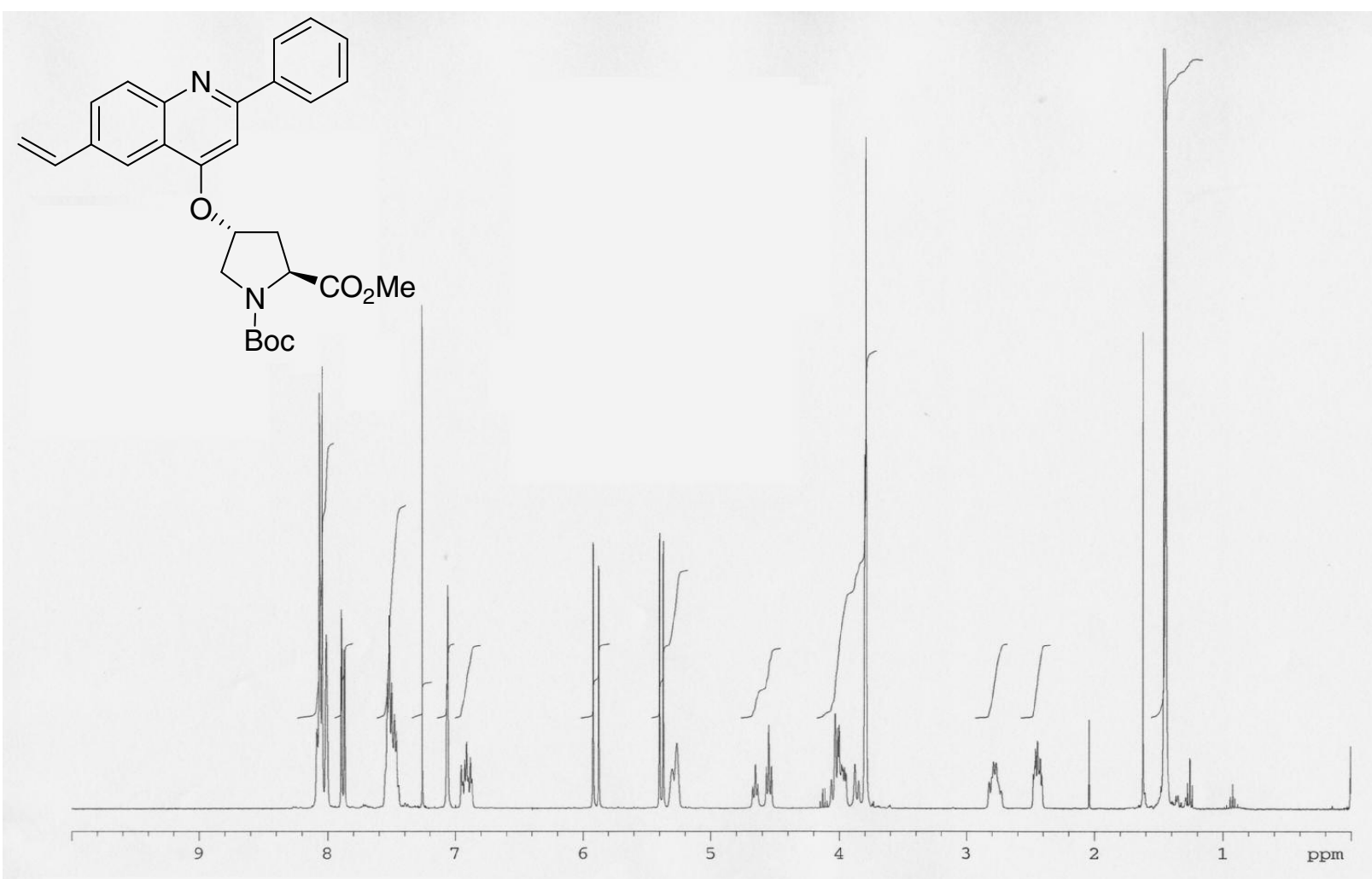

Method A HPLC Data

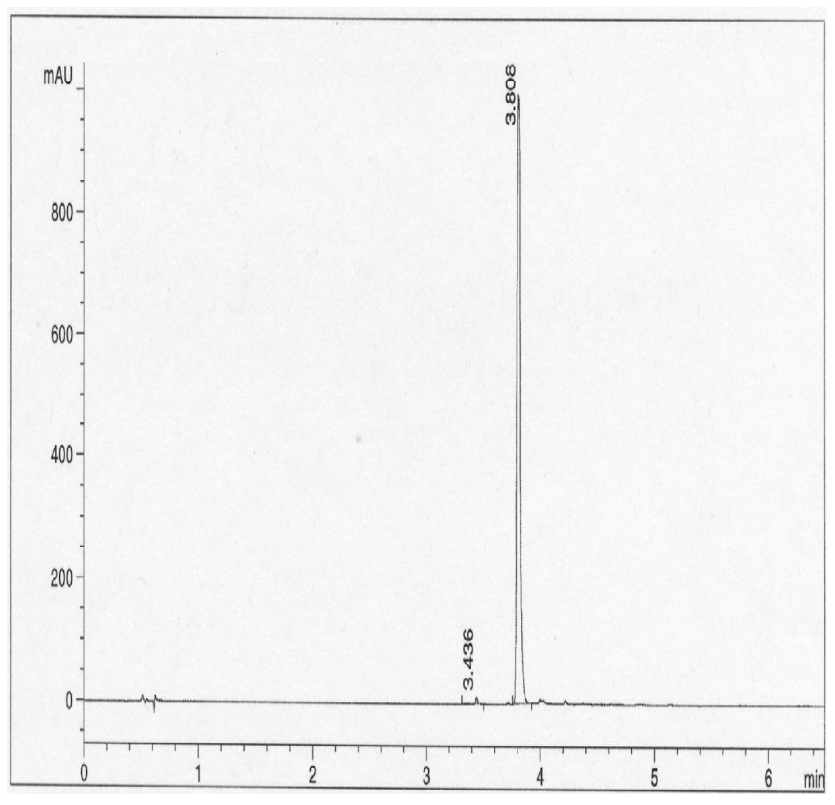

Method B HPLC Data

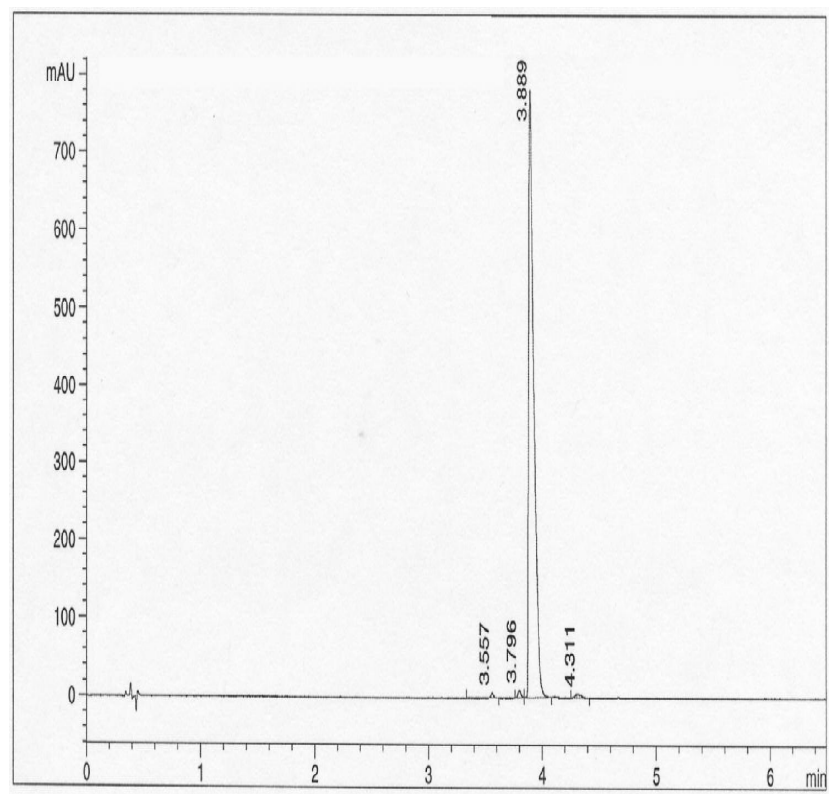


NMR and HPLC Data for Compound 8b

${ }^{1} \mathrm{H}$ NMR Data $\left(400 \mathrm{MHz}, \mathrm{CD}_{3} \mathrm{OD}\right)$

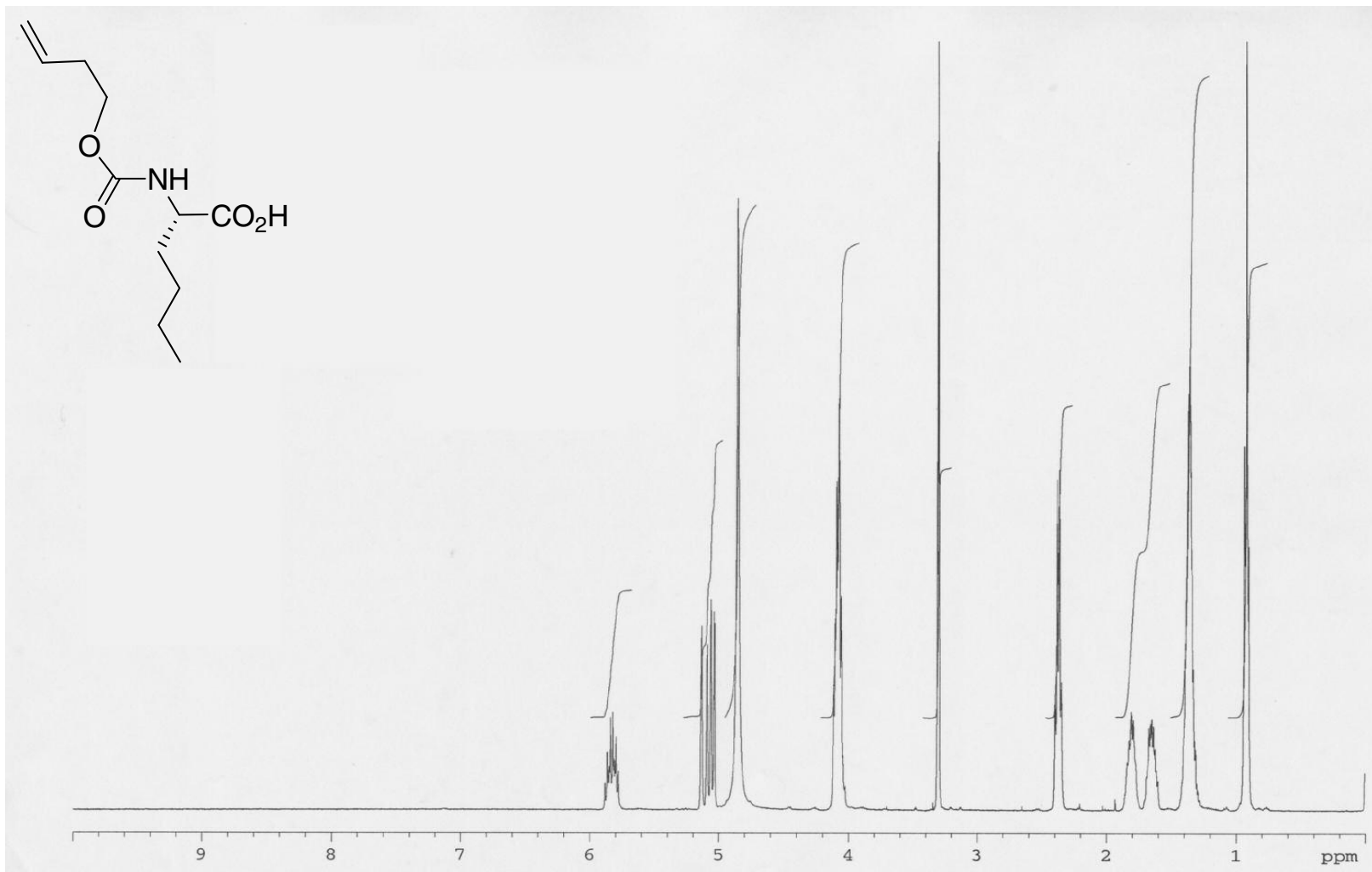


NMR and HPLC Data for Compound 8c

${ }^{1} \mathrm{H}$ NMR Data $\left(400 \mathrm{MHz}, \mathrm{CD}_{3} \mathrm{OD}\right)$

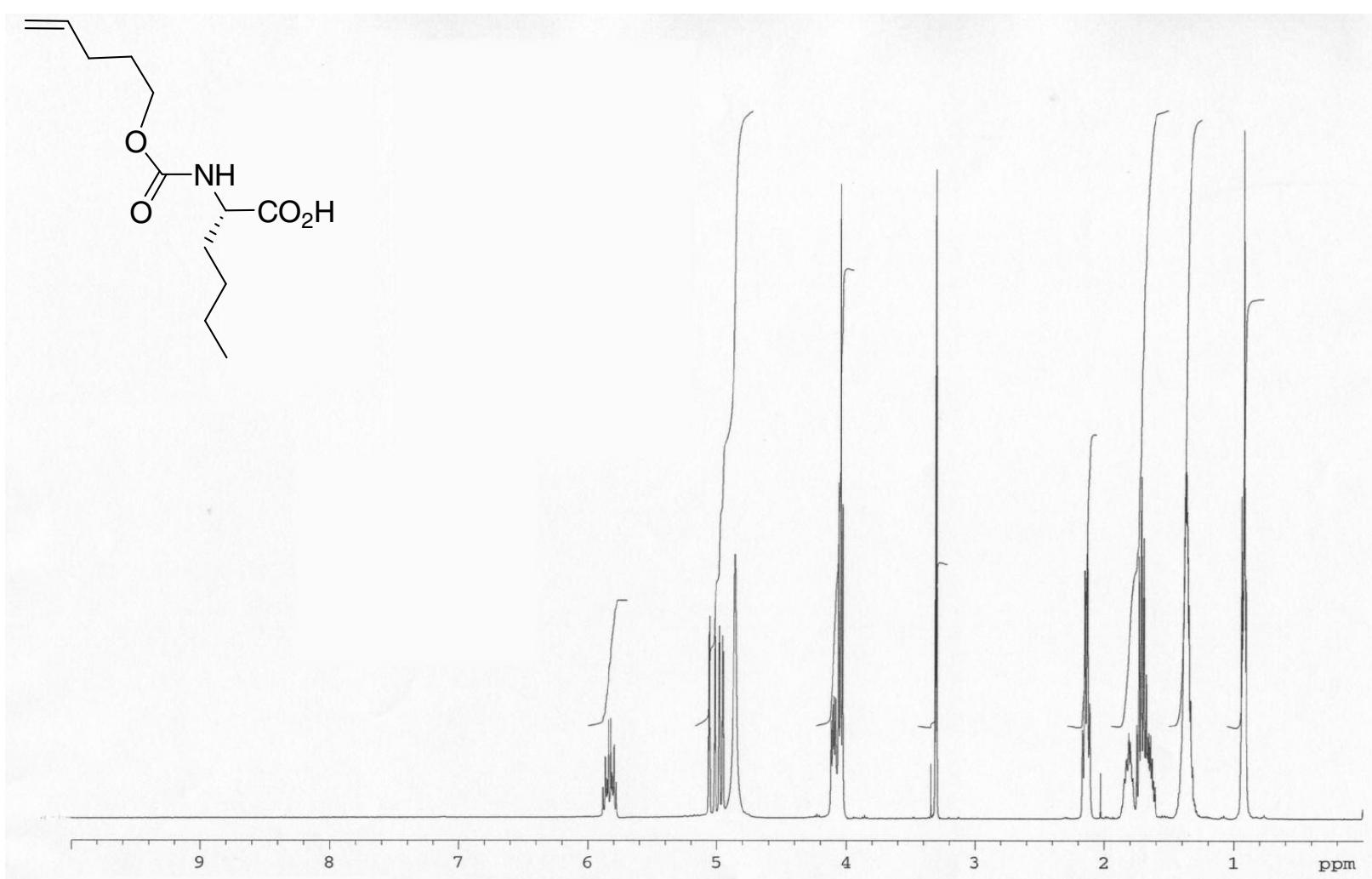

Method A HPLC Data

Method B HPLC Data 


\section{NMR and HPLC Data for Compound 8d}

${ }^{1} \mathrm{H}$ NMR Data $\left(400 \mathrm{MHz}, \mathrm{CD}_{3} \mathrm{OD}\right)$

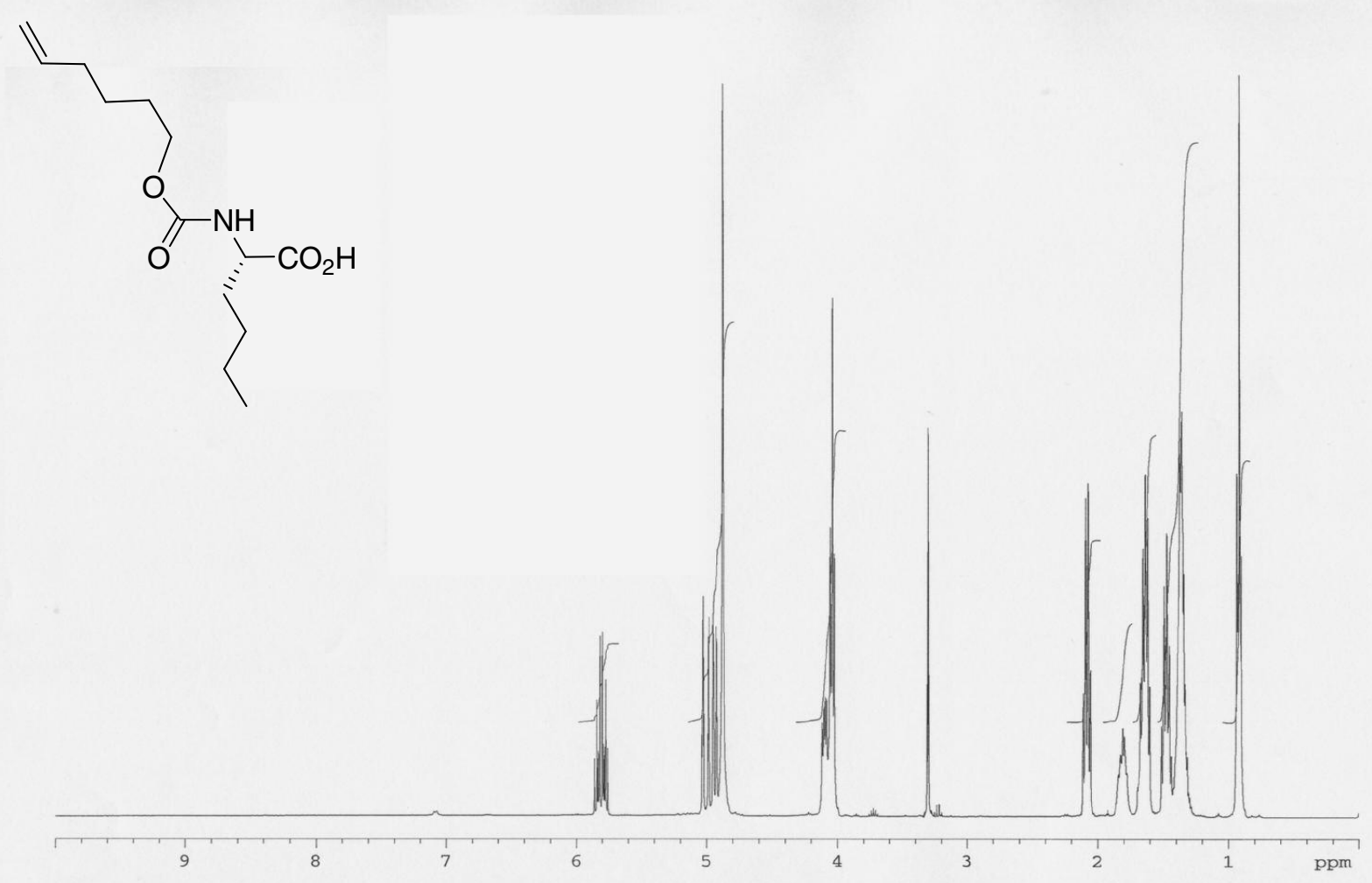

Method A HPLC Data

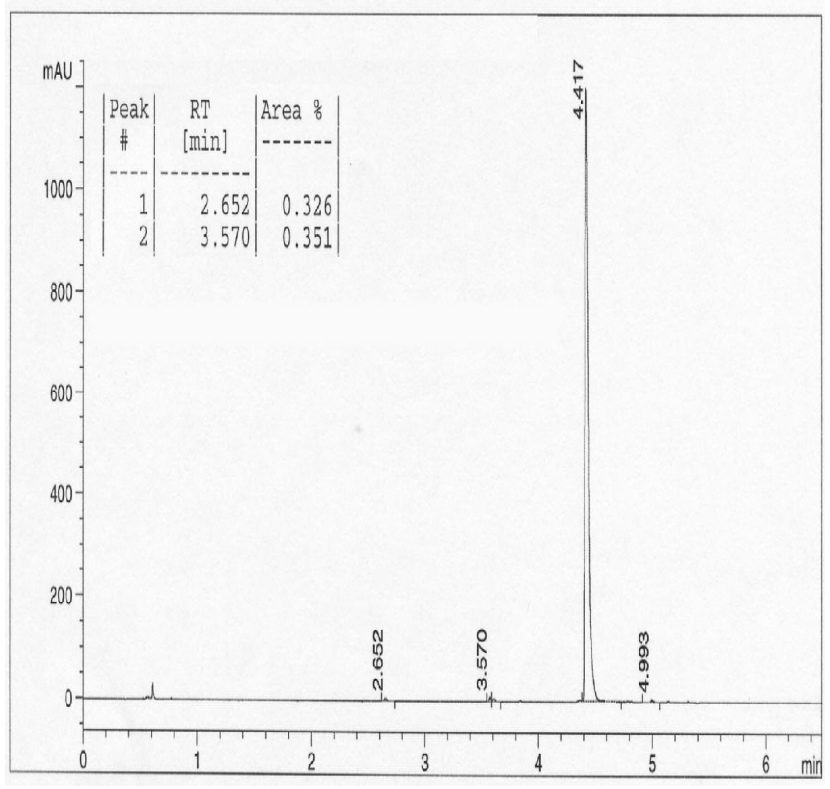

Method B HPLC Data

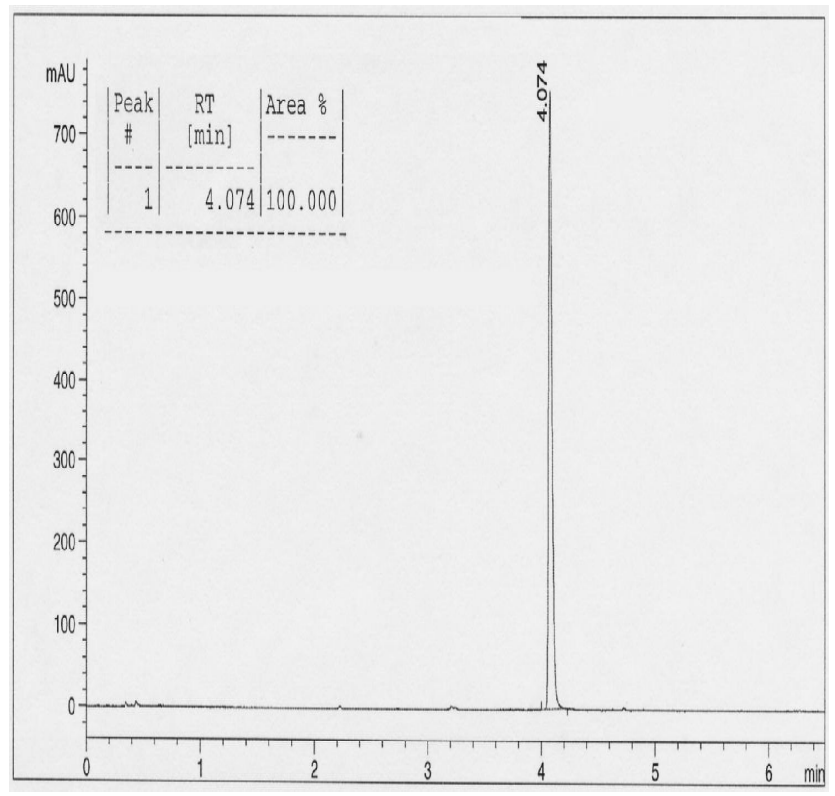


NMR and HPLC Data for Compound 9a

${ }^{1} \mathrm{H}$ NMR Data $\left(400 \mathrm{MHz}, \mathrm{CDCl}_{3}\right.$ )

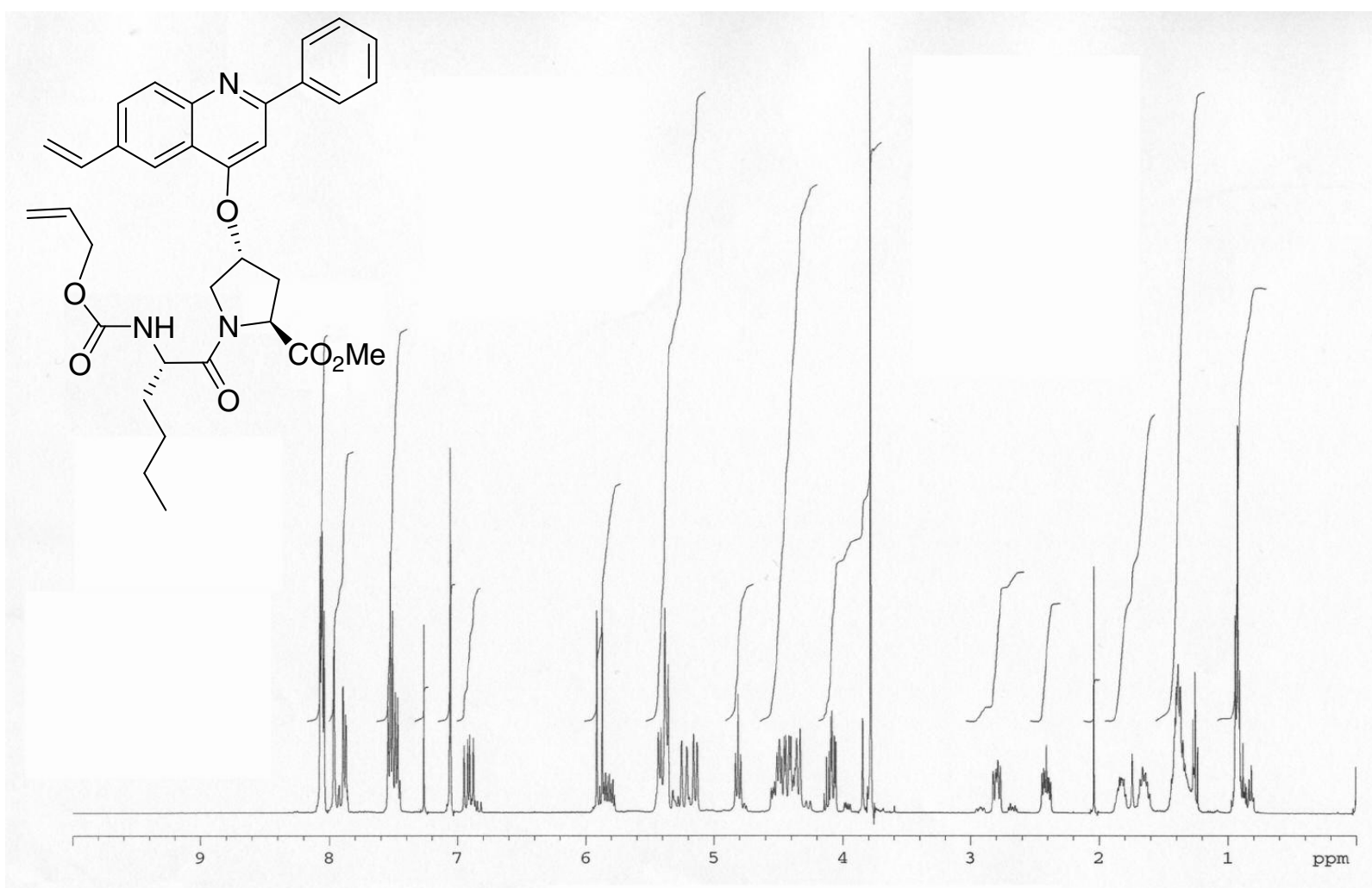

Method A HPLC Data

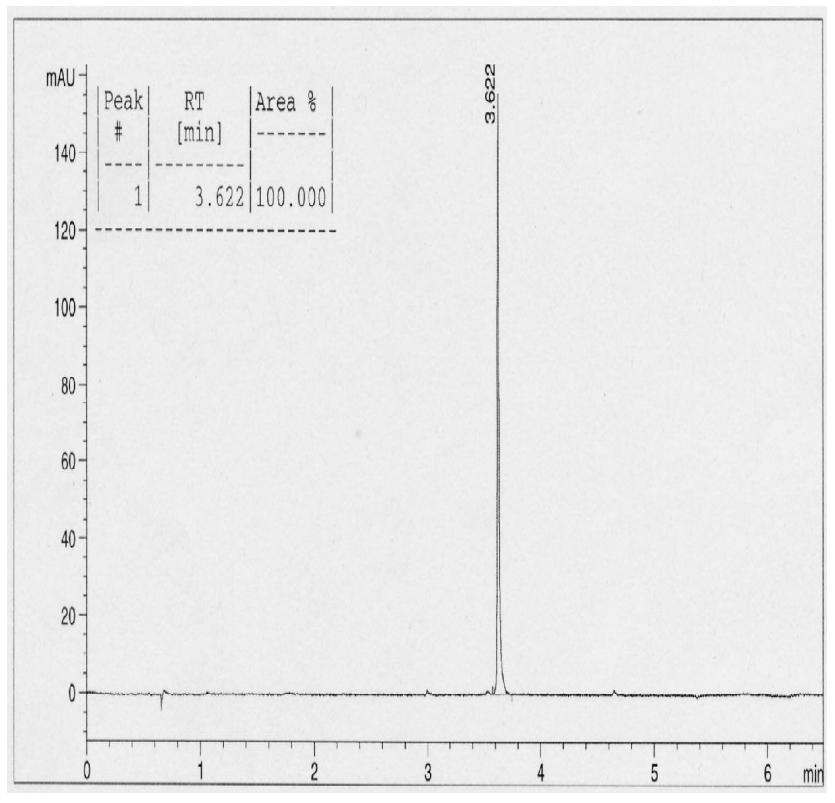

Method B HPLC Data

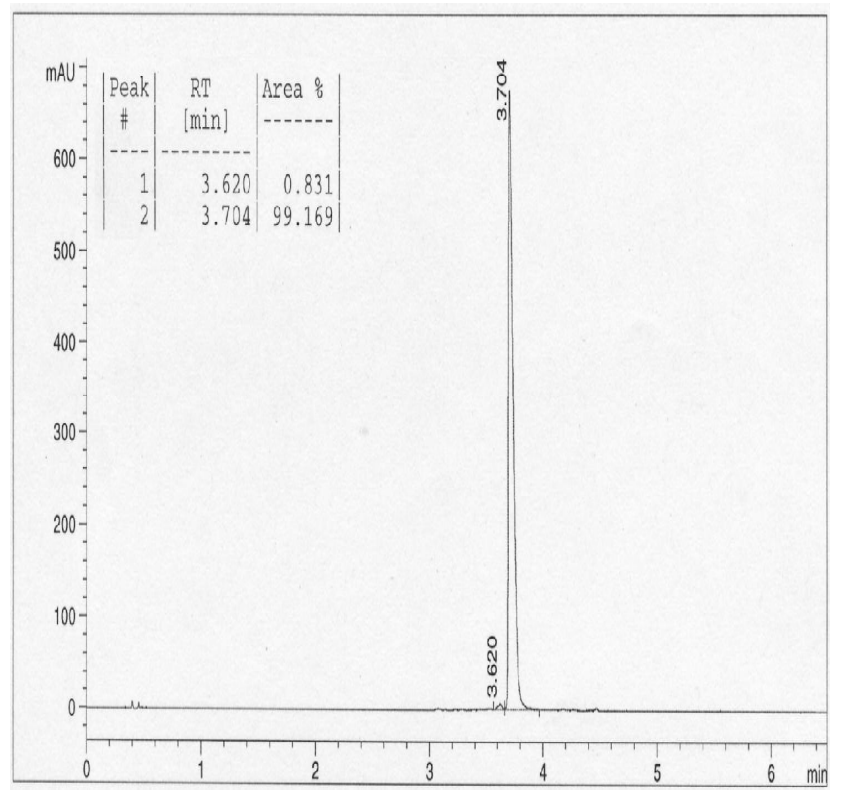


NMR and HPLC Data for Compound 9b

${ }^{1} \mathrm{H}$ NMR Data $\left(400 \mathrm{MHz}, \mathrm{CDCl}_{3}\right.$ )

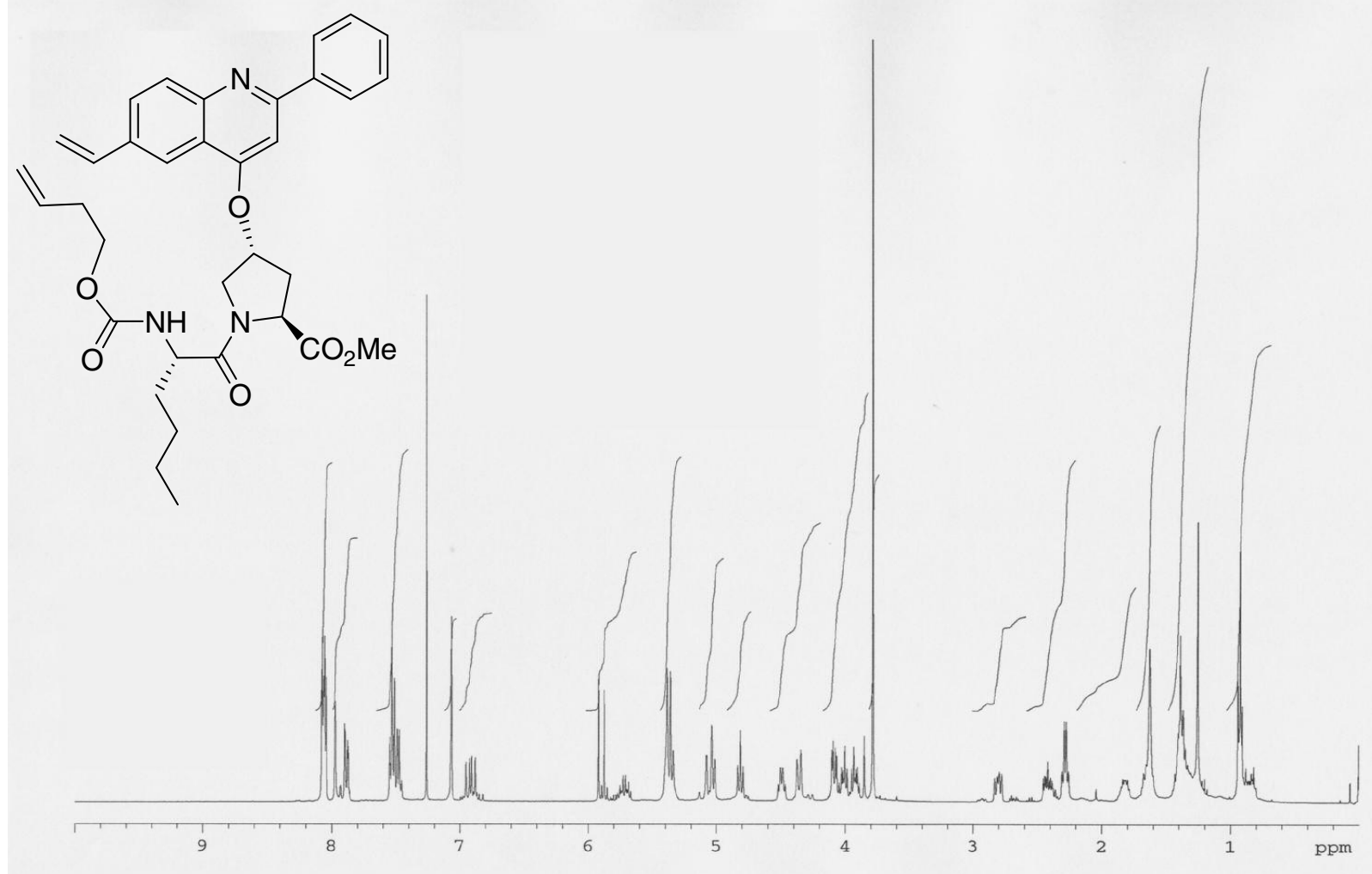

Method A HPLC Data

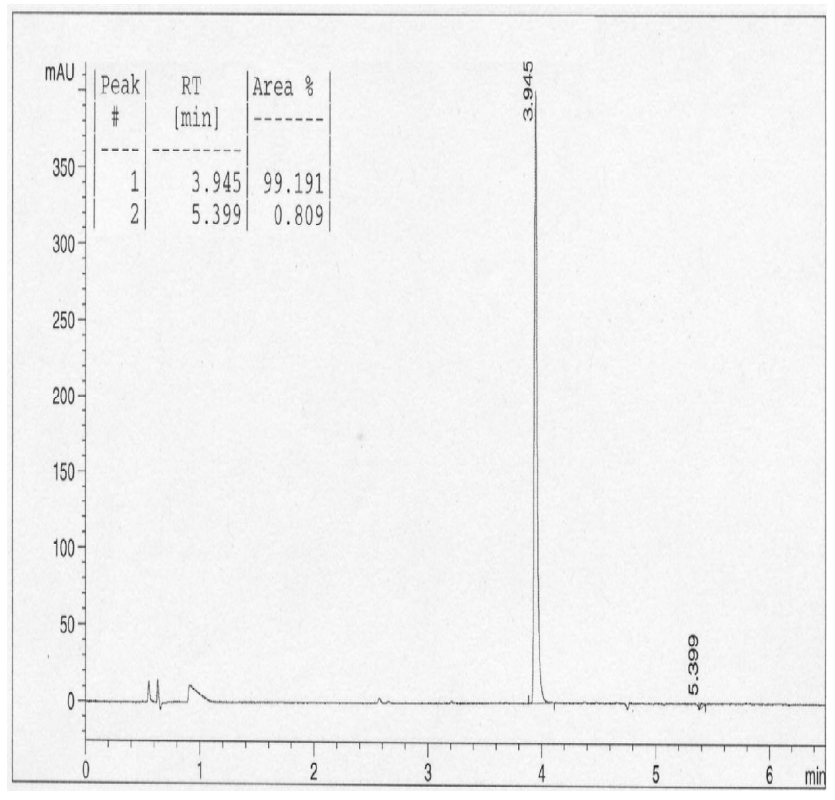

Method B HPLC Data

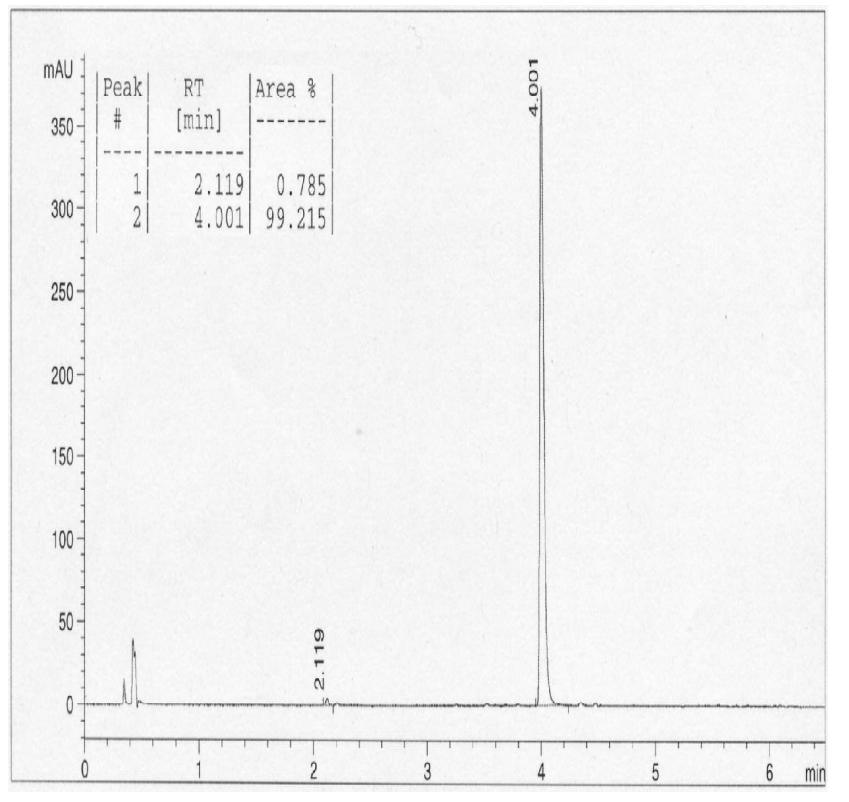


NMR and HPLC Data for Compound 9c

${ }^{1} \mathrm{H}$ NMR Data $\left(400 \mathrm{MHz}, \mathrm{CDCl}_{3}\right)$

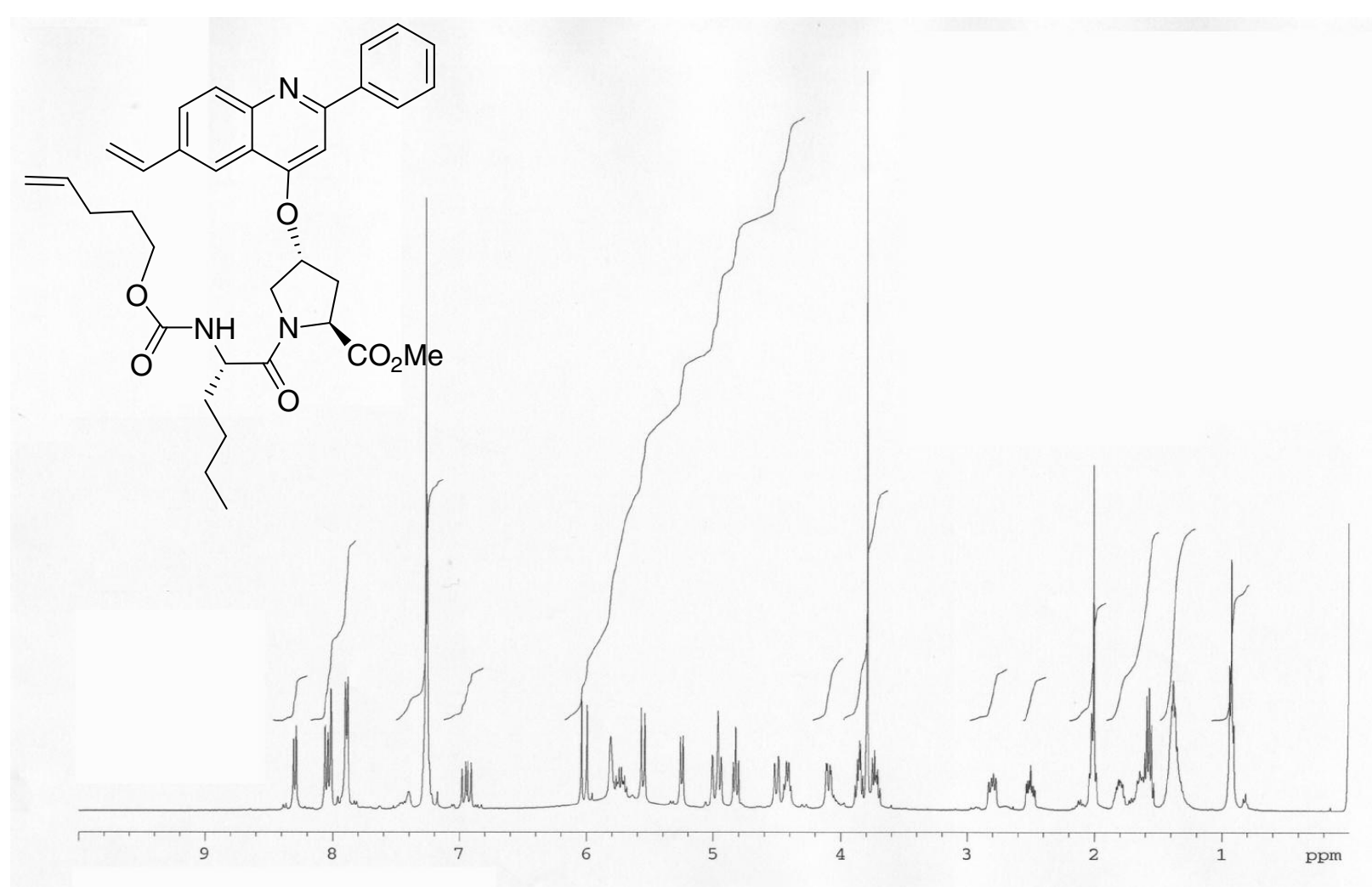

Method A HPLC Data

Method B HPLC Data 


\section{NMR and HPLC Data for Compound 9d}

${ }^{1} \mathrm{H}$ NMR Data $\left(400 \mathrm{MHz}, \mathrm{CDCl}_{3}\right)$

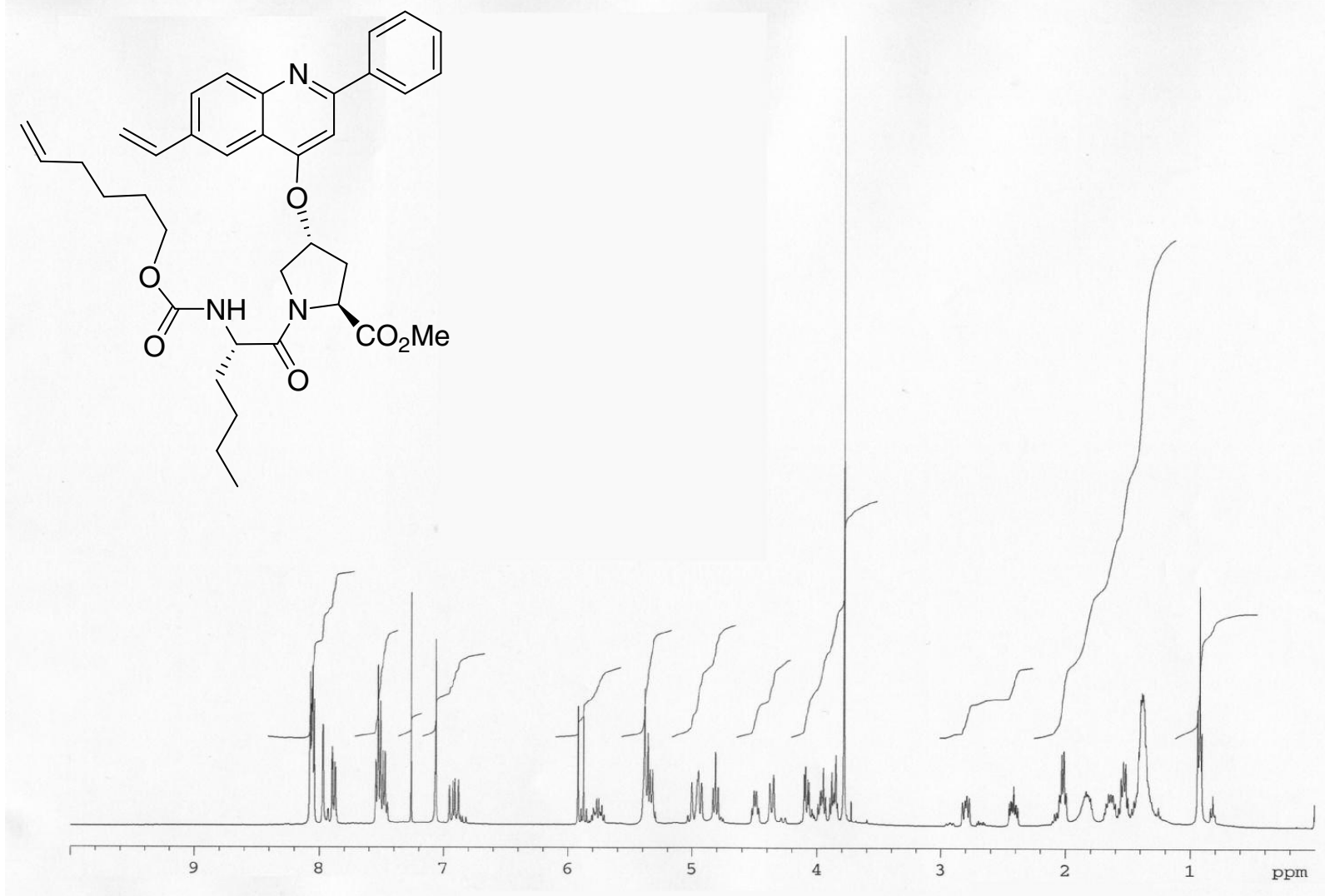

Method A HPLC Data

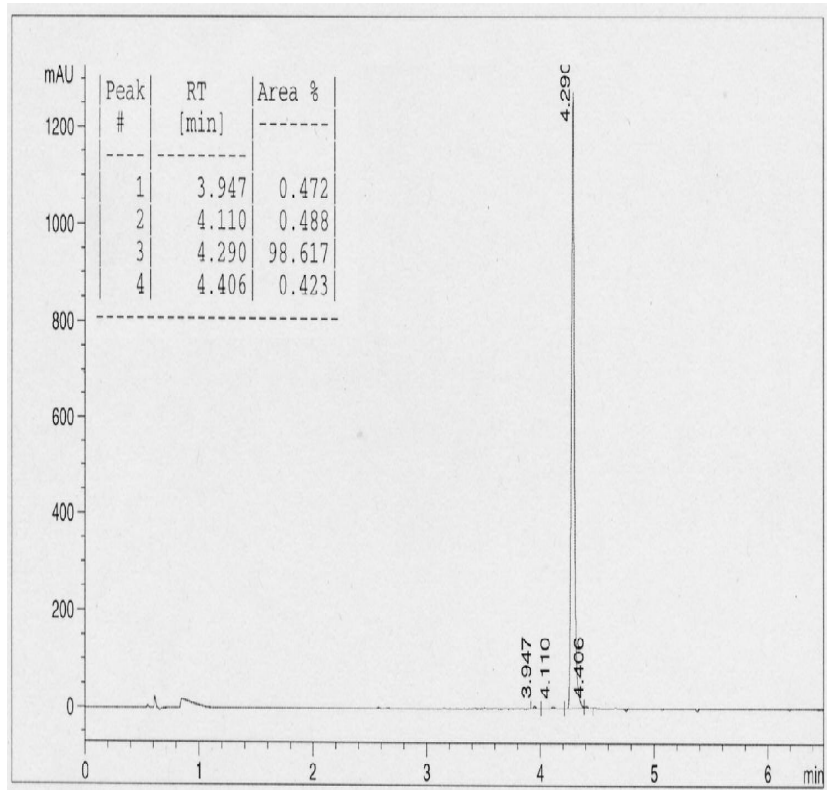

Method B HPLC Data

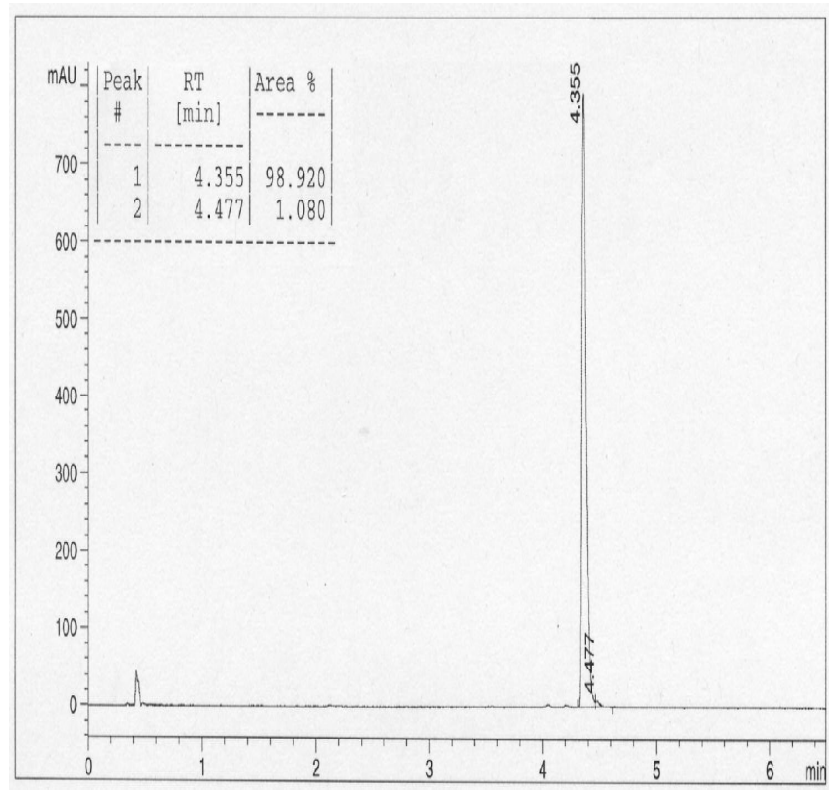


NMR and HPLC Data for Compound 10a

${ }^{1} \mathrm{H}$ NMR Data $\left(400 \mathrm{MHz}, \mathrm{CDCl}_{3}\right.$ )

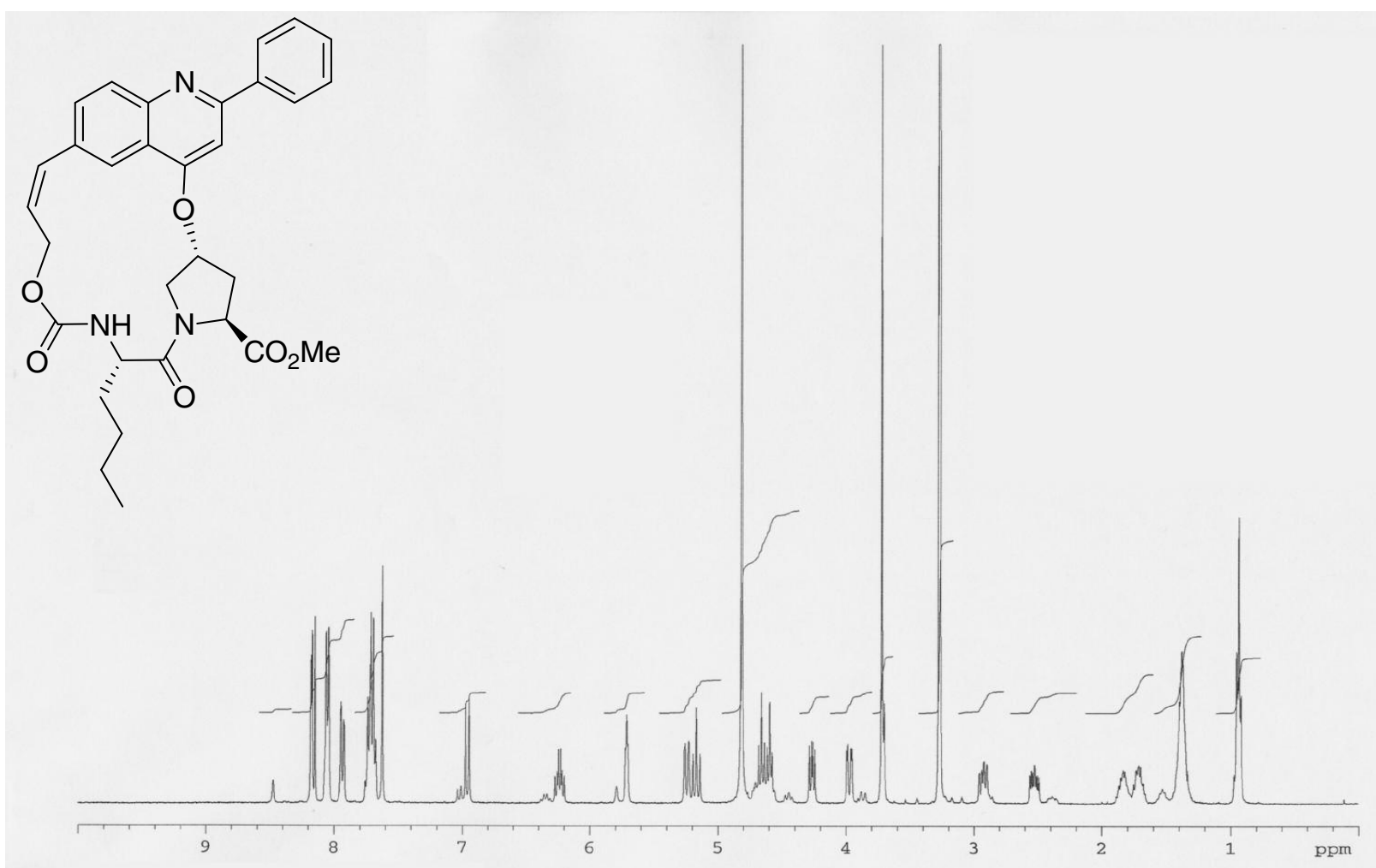

Method A HPLC Data

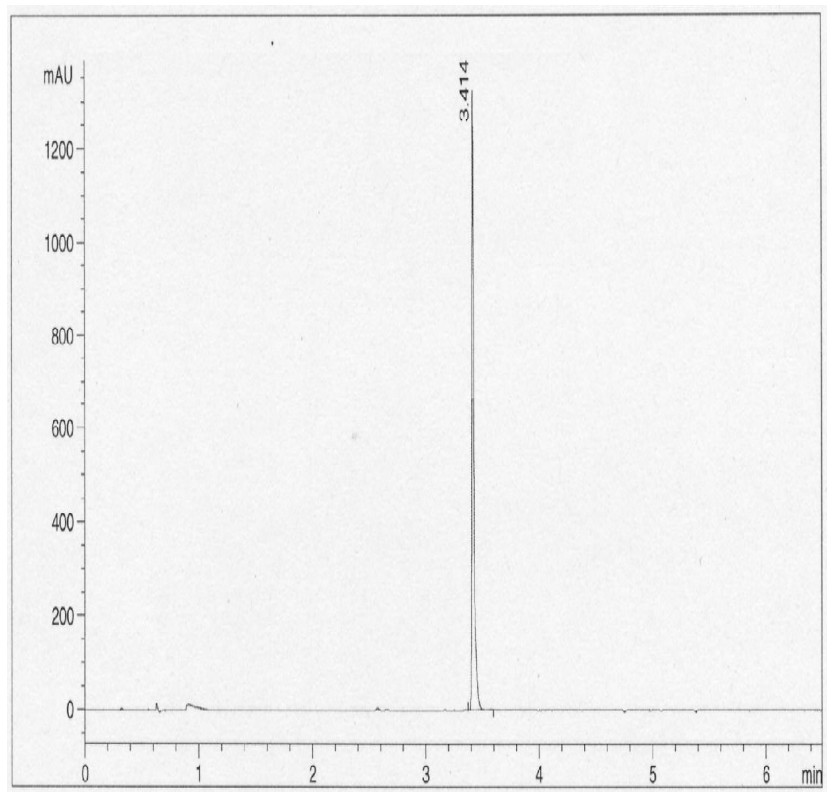

Method B HPLC Data

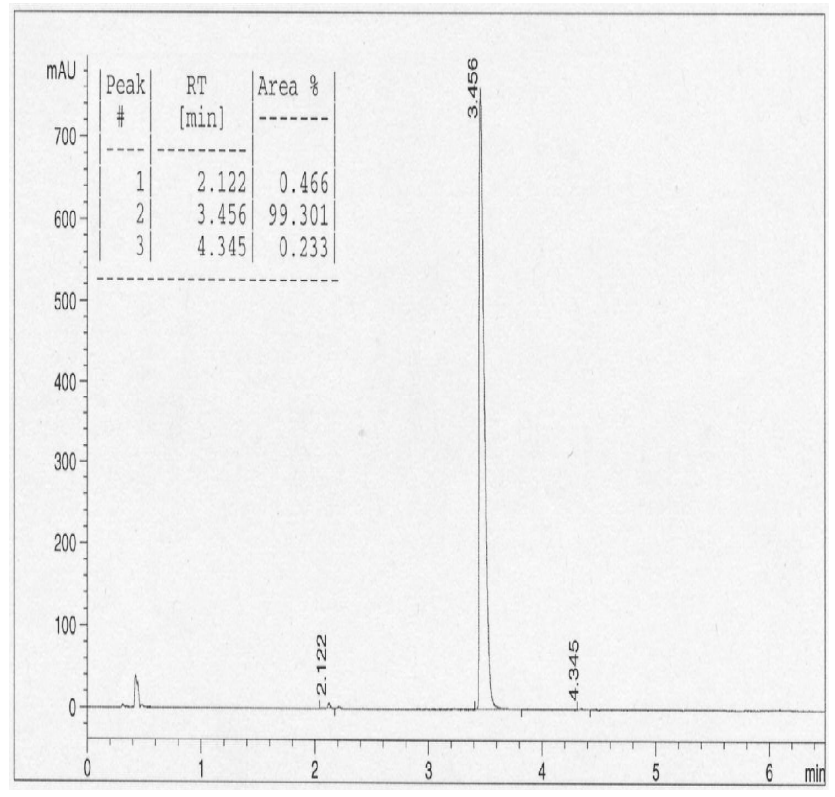


NMR and HPLC Data for Compound 10b

${ }^{1} \mathrm{H}$ NMR Data $\left(400 \mathrm{MHz}, \mathrm{CDCl}_{3}\right.$ )

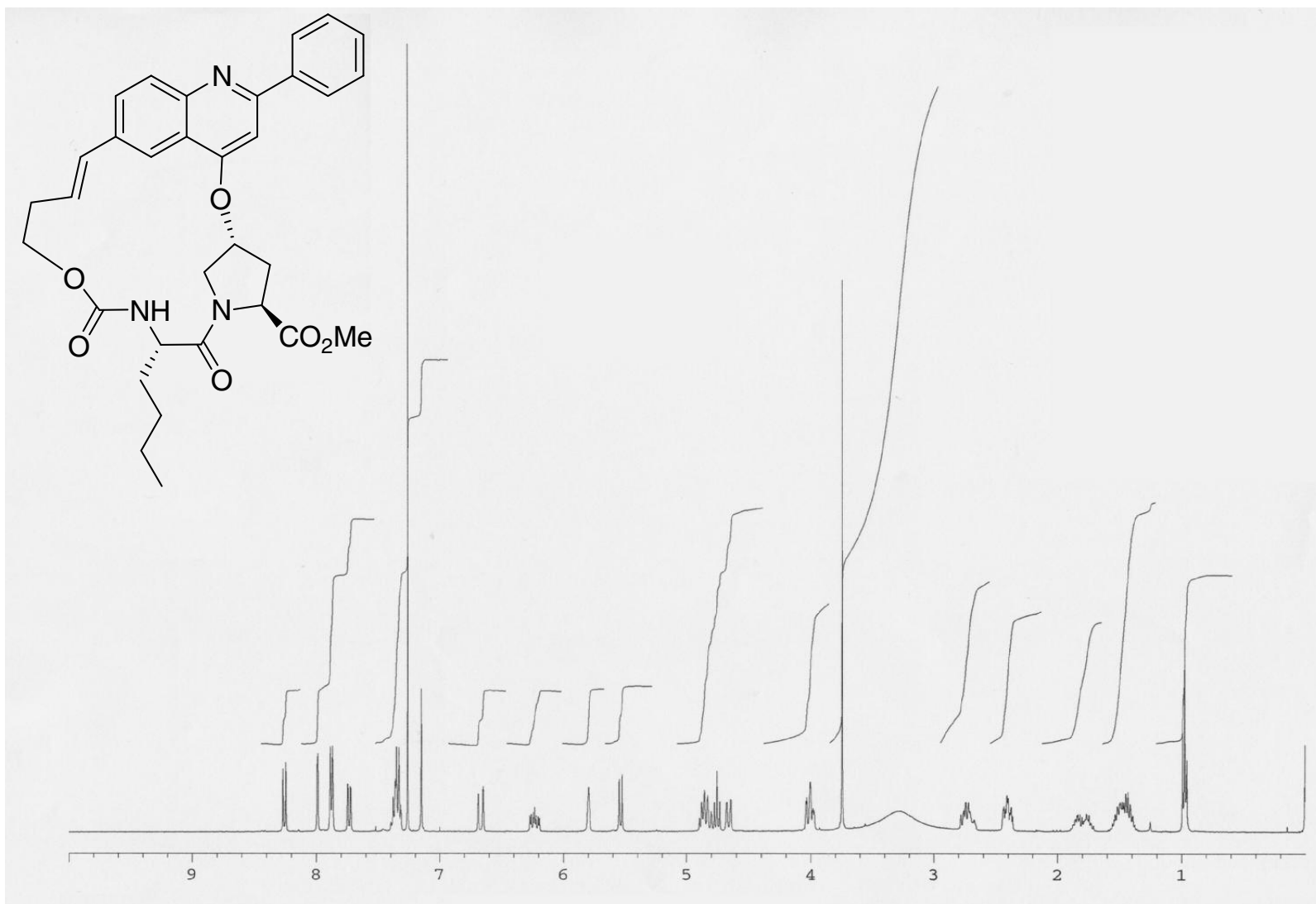

Method A HPLC Data

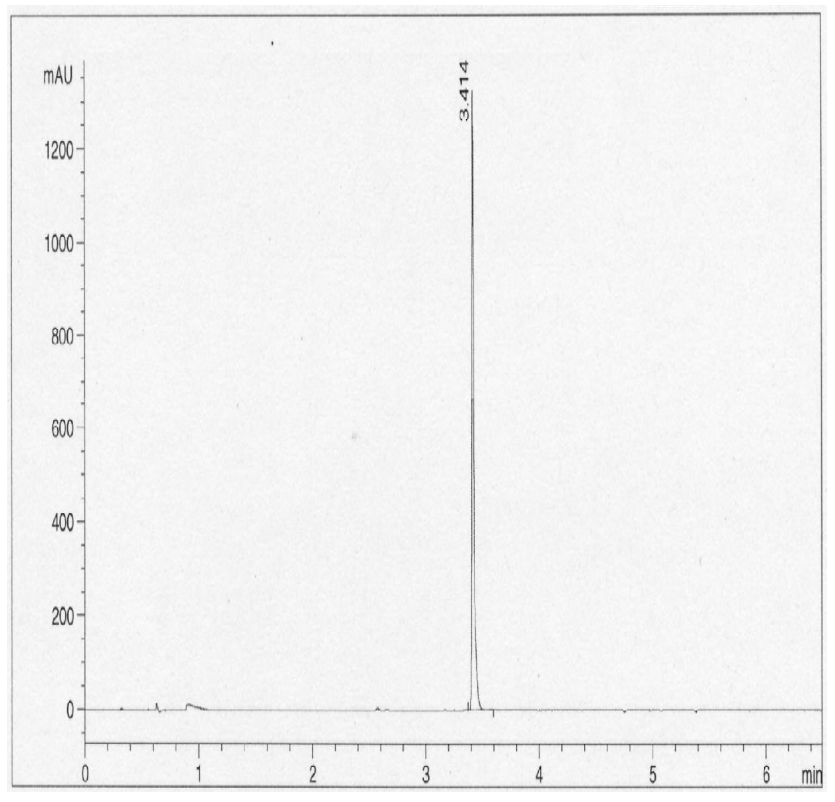

Method B HPLC Data

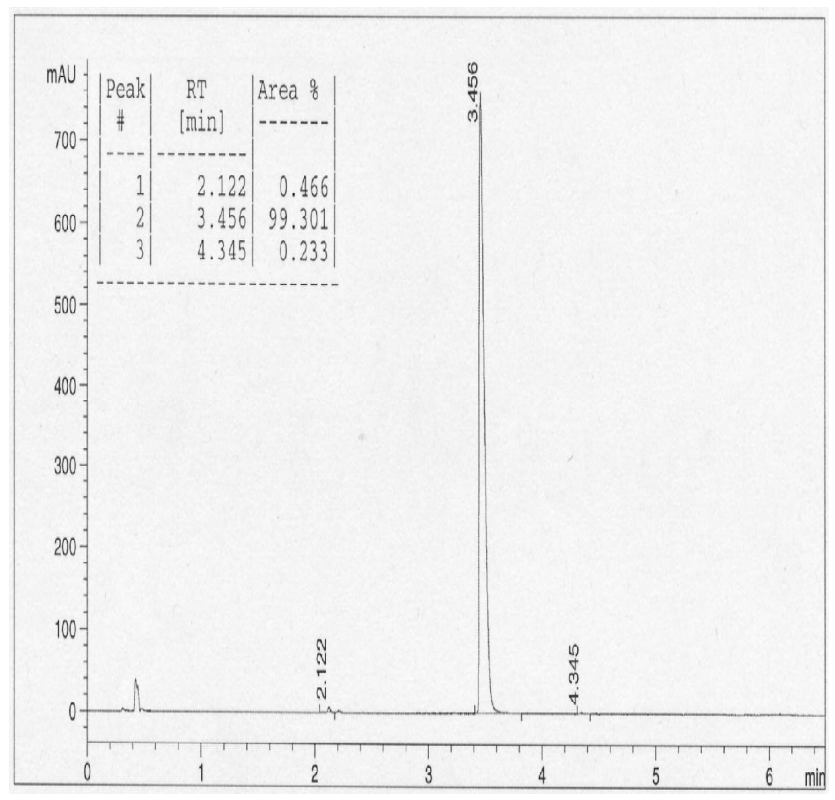


NMR and HPLC Data for Compound 10c

${ }^{1} \mathrm{H}$ NMR Data $\left(400 \mathrm{MHz}, \mathrm{CDCl}_{3}\right.$ )

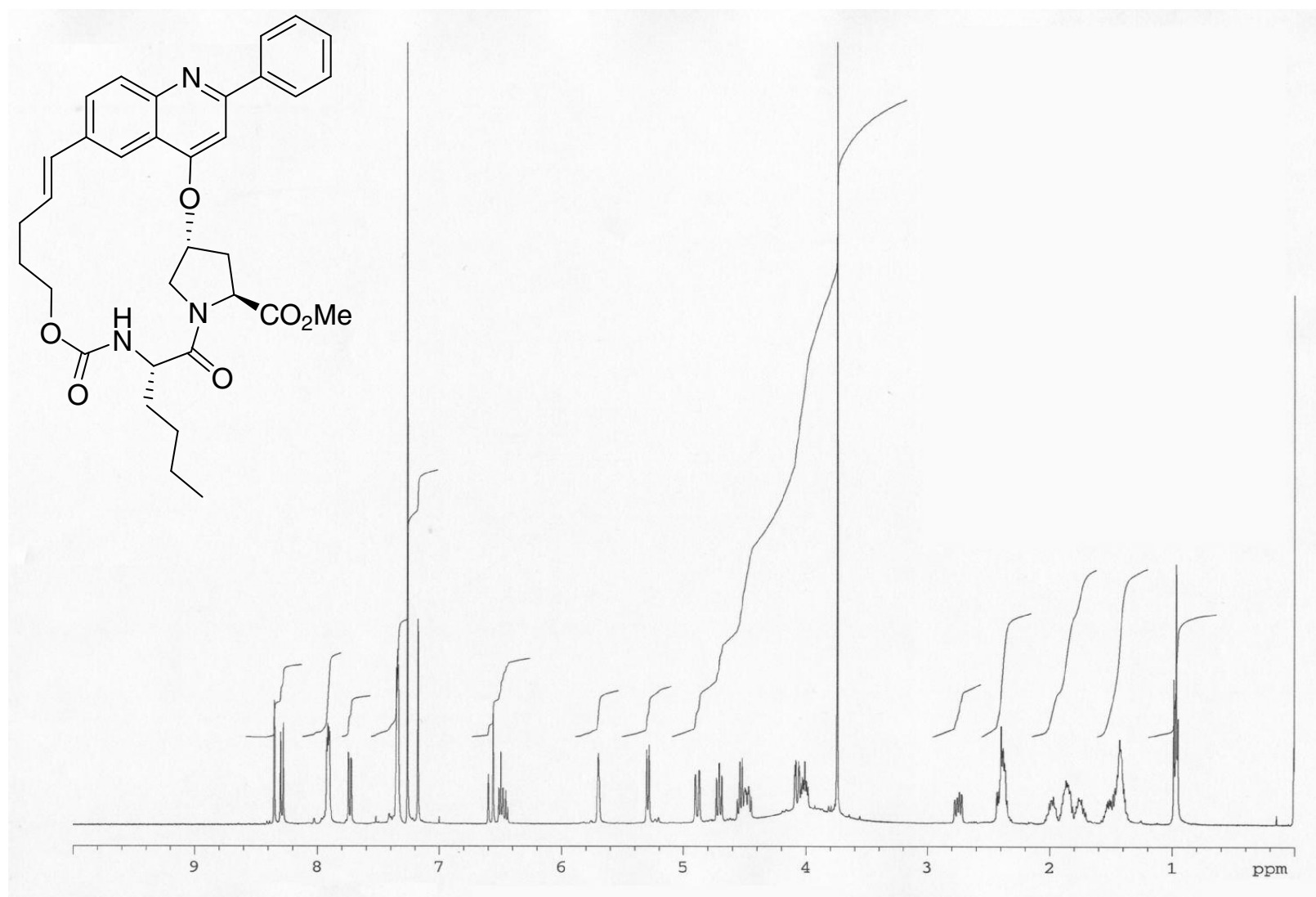

Method A HPLC Data

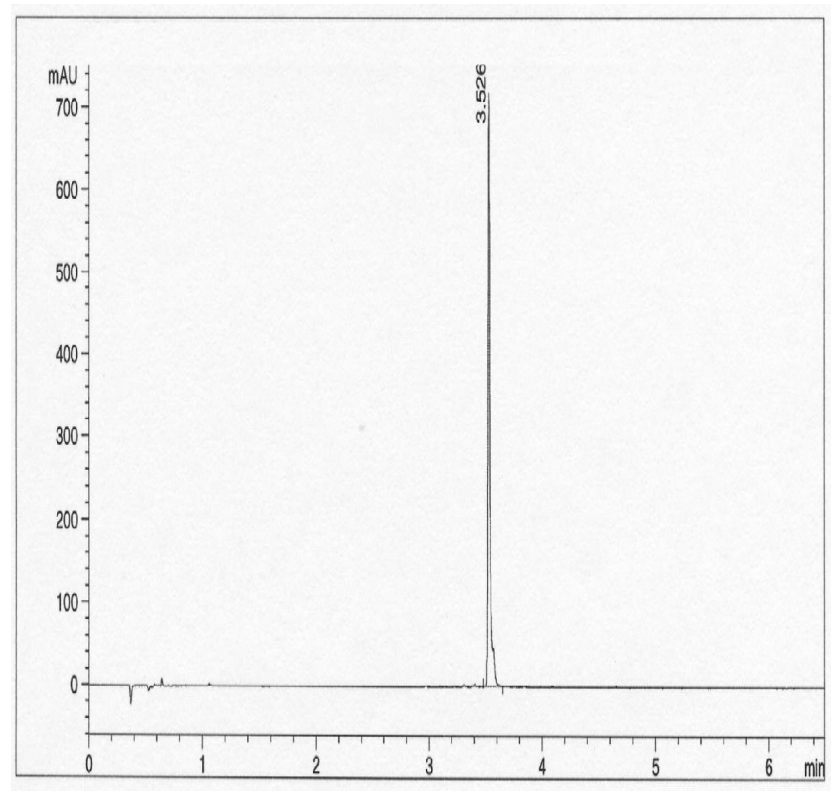

Method B HPLC Data

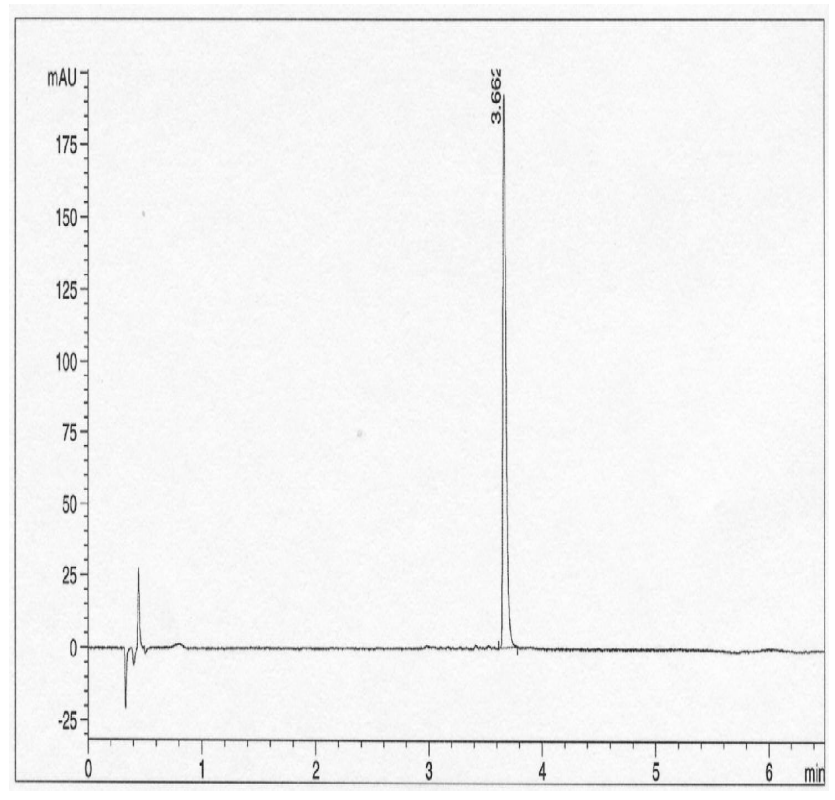




\section{NMR and HPLC Data for Compound 10d}

${ }^{1} \mathrm{H}$ NMR Data $\left(400 \mathrm{MHz}, \mathrm{CDCl}_{3}\right)$

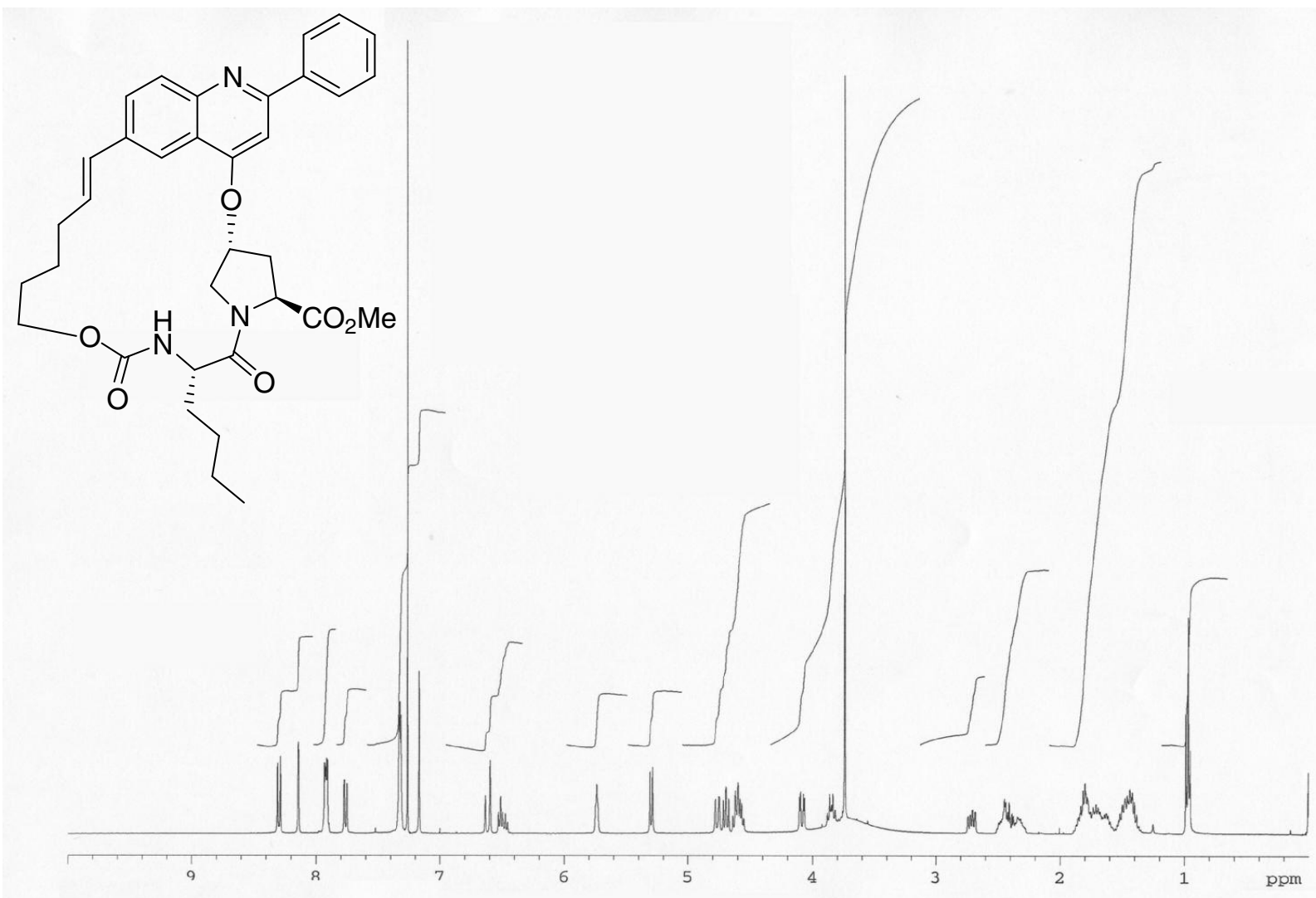

Method A HPLC Data

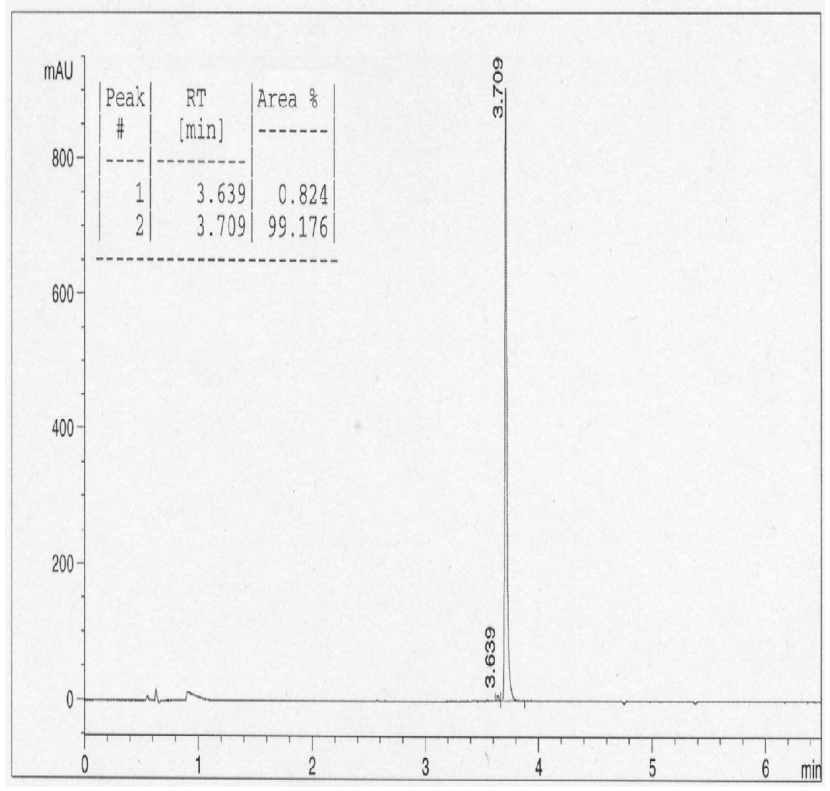

Method B HPLC Data

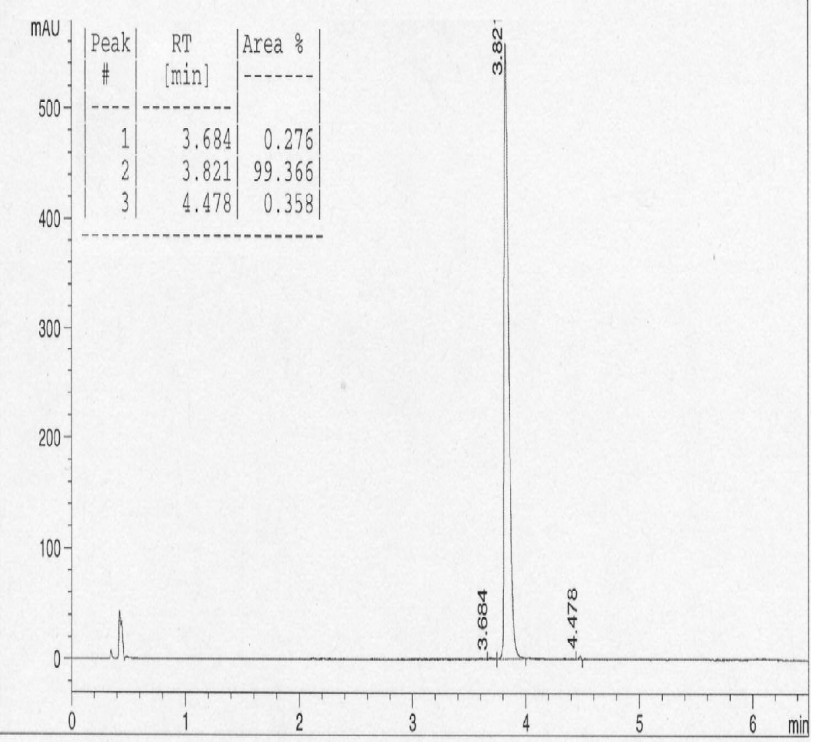


NMR and HPLC Data for Compound 12a

${ }^{1} \mathrm{H}$ NMR Data $\left(400 \mathrm{MHz}, \mathrm{CDCl}_{3}\right.$ )

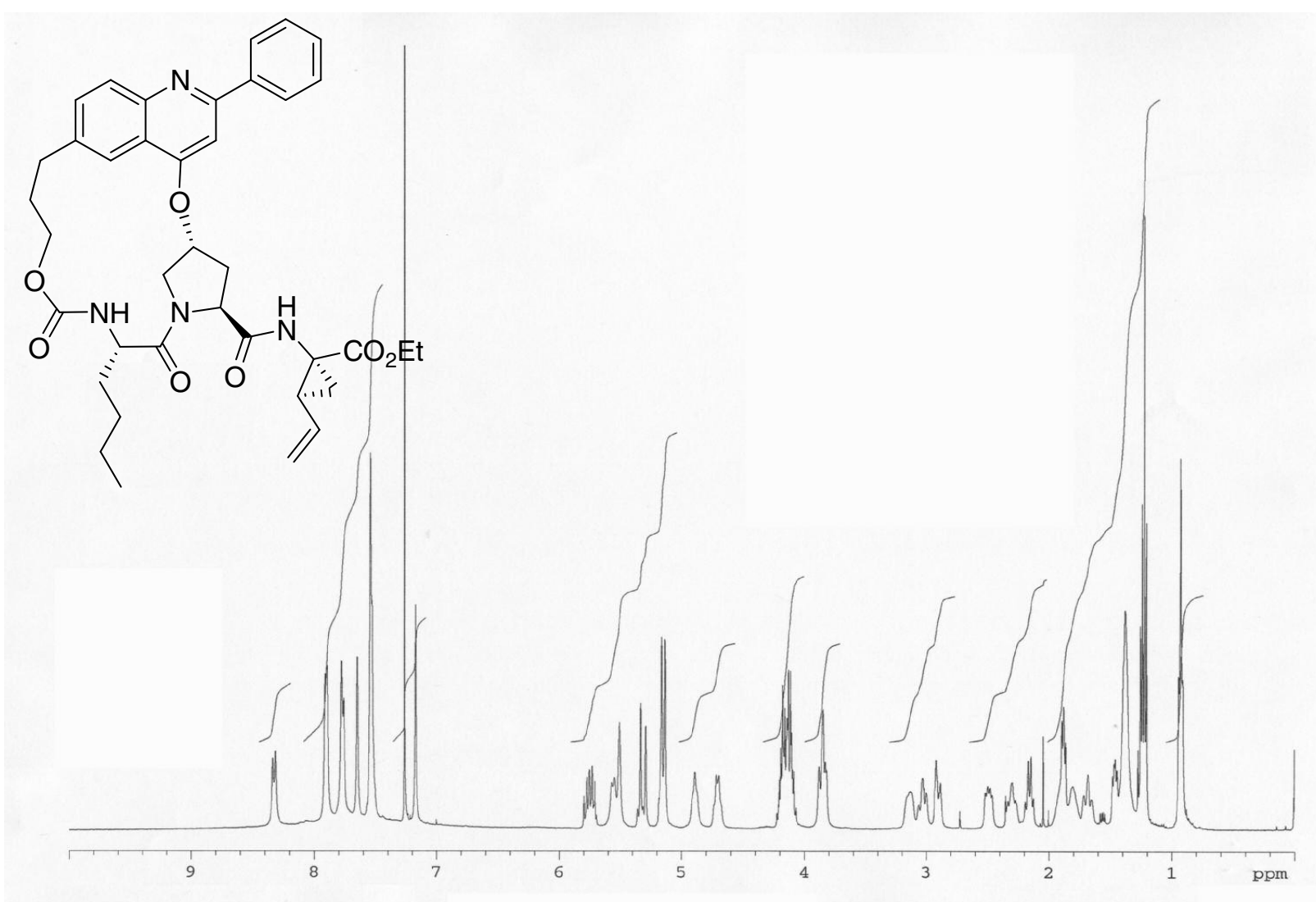

Method A HPLC Data

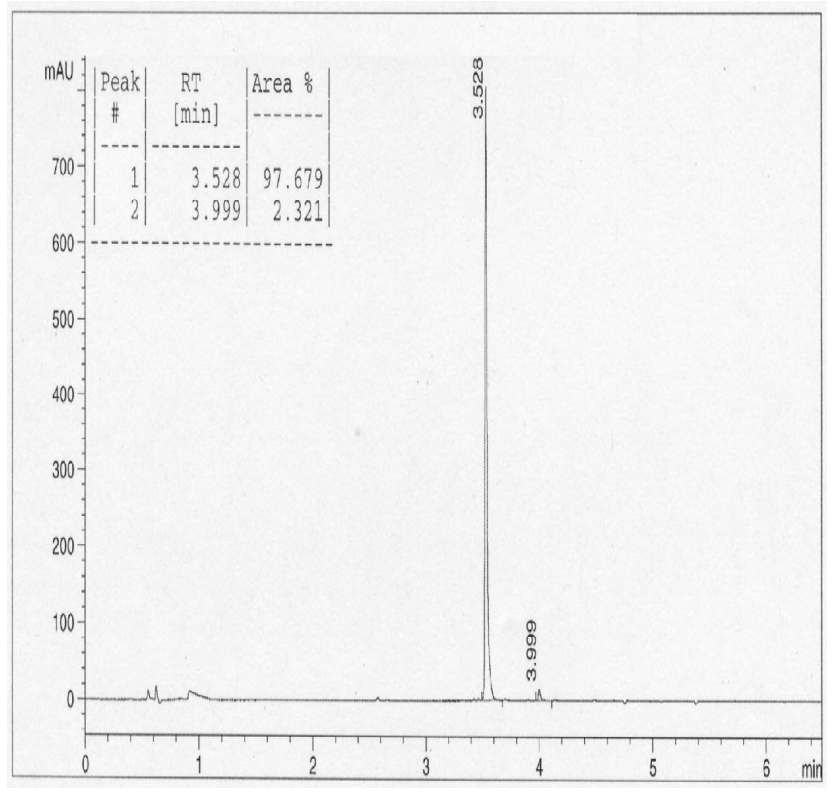

\section{Method B HPLC Data}

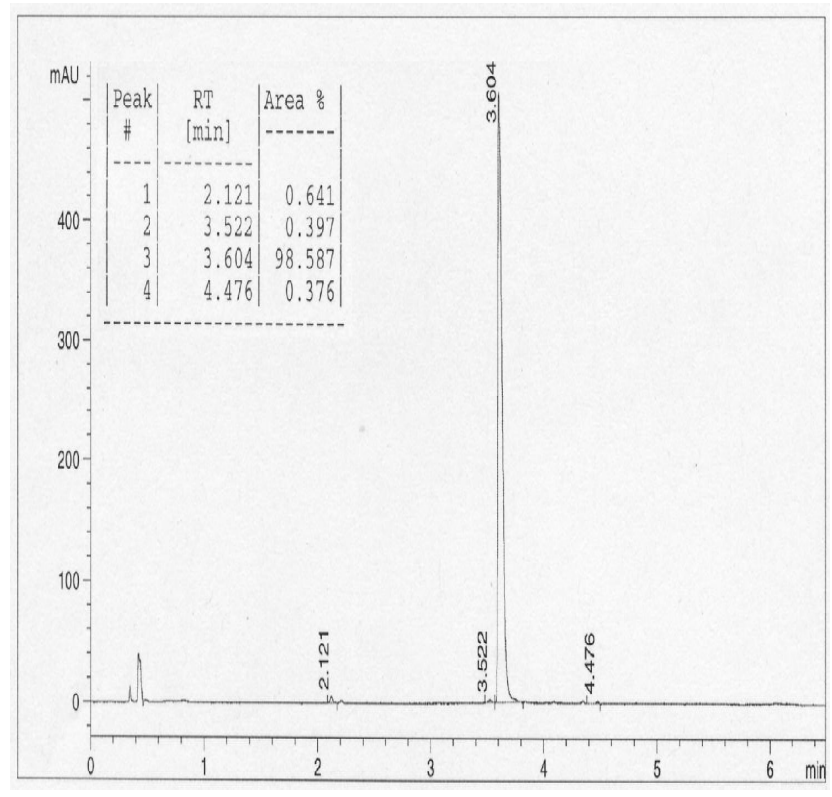


NMR and HPLC Data for Compound 12b

${ }^{1} \mathrm{H}$ NMR Data $\left(400 \mathrm{MHz}, \mathrm{CDCl}_{3}\right)$

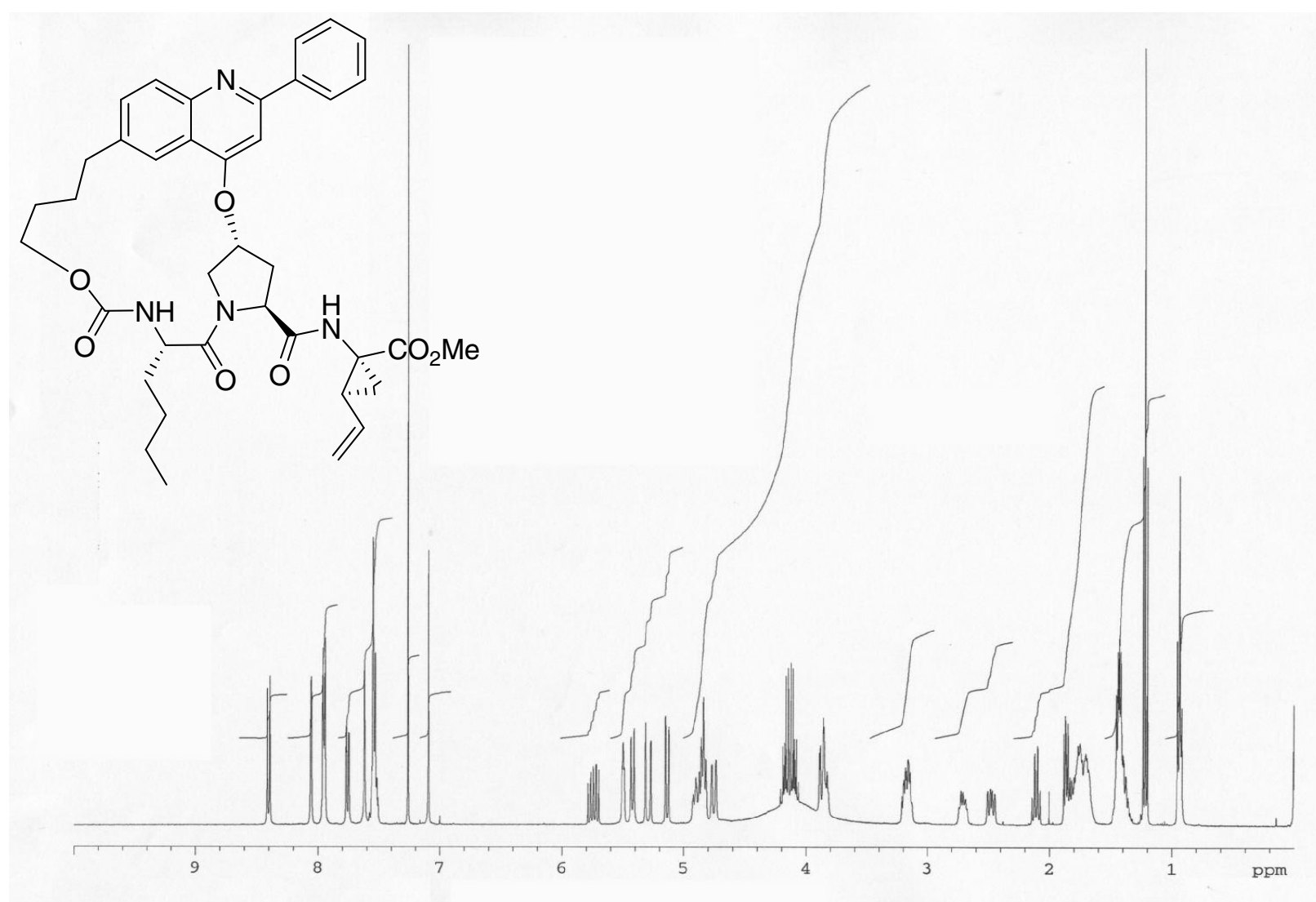

Method A HPLC Data

Method B HPLC Data 


\section{NMR and HPLC Data for Compound 12c}

${ }^{1} \mathrm{H}$ NMR Data $\left(400 \mathrm{MHz}, \mathrm{CDCl}_{3}\right)$

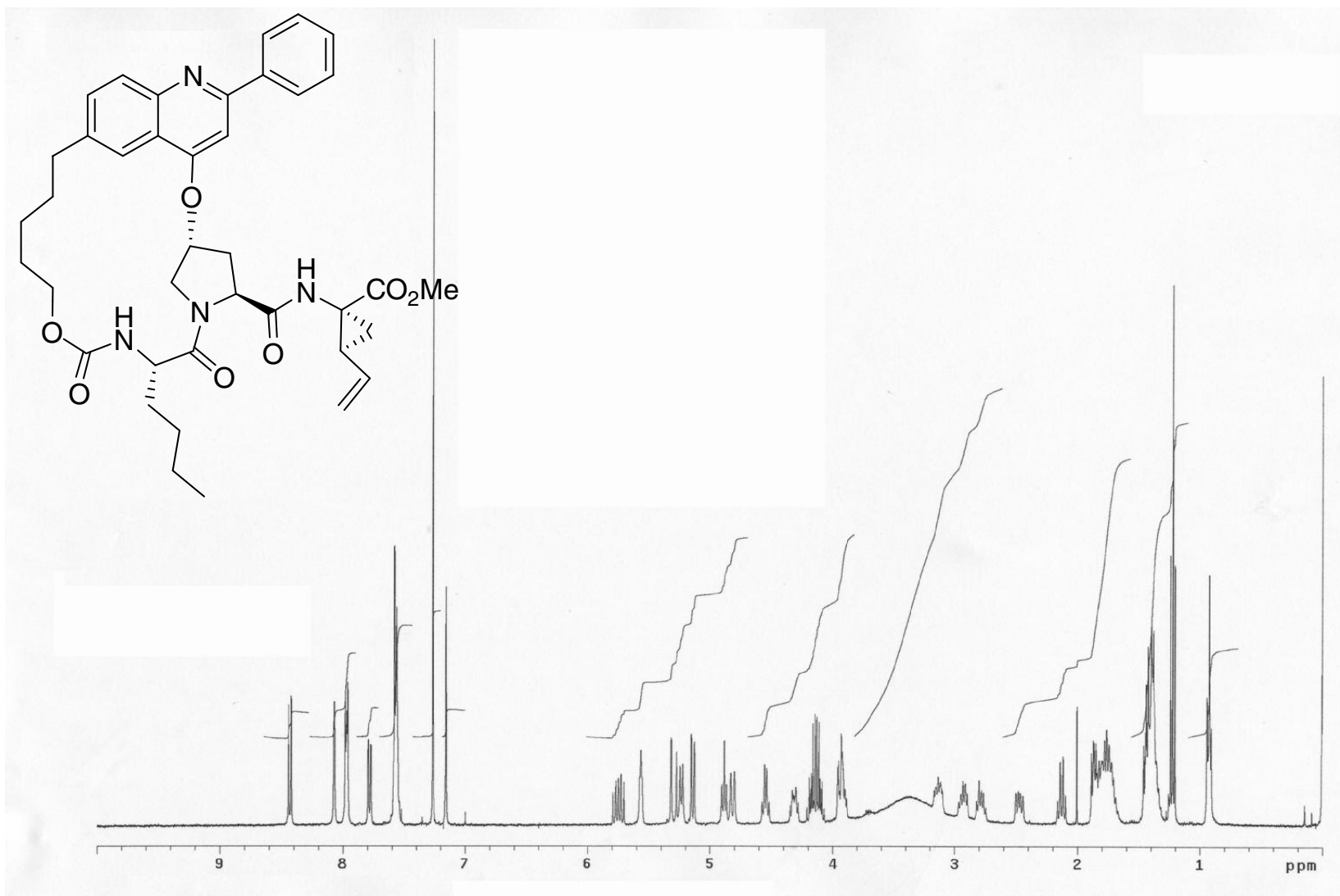

\section{Method A HPLC Data}

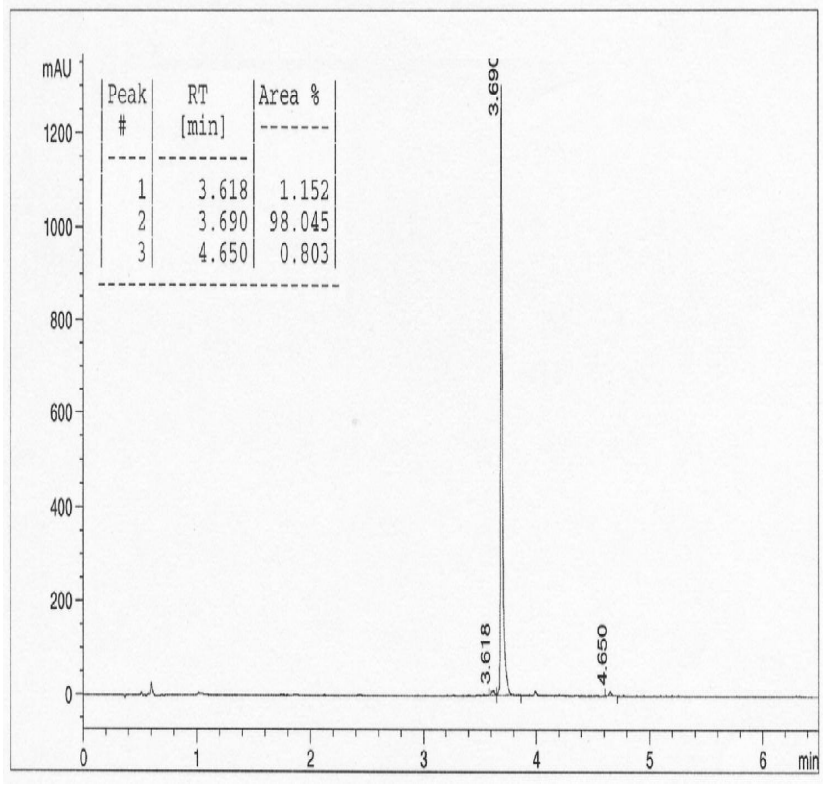

Method B HPLC Data

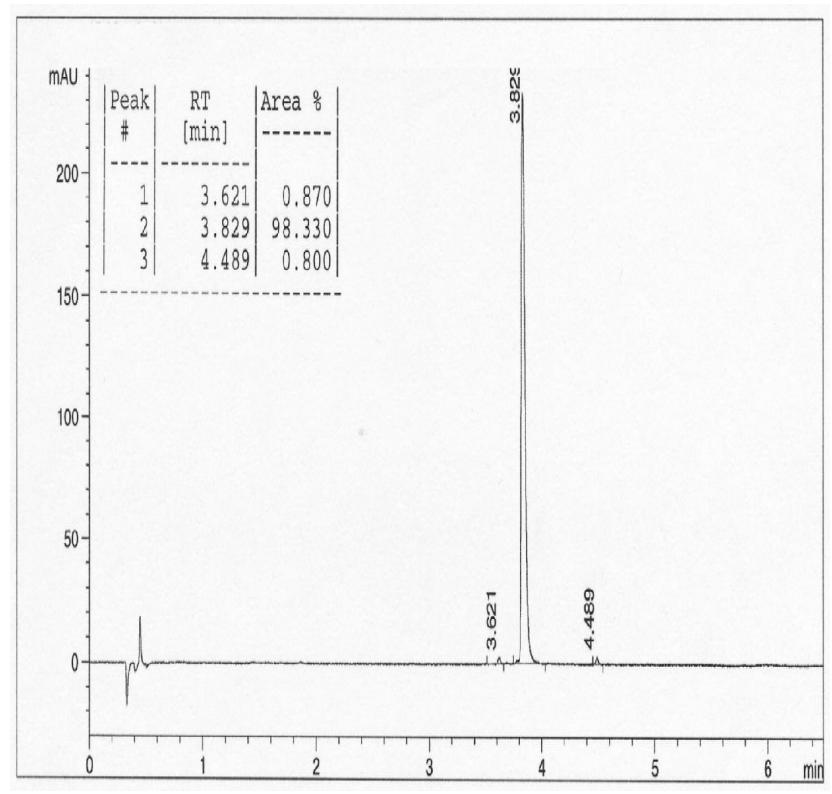


NMR and HPLC Data for Compound 12d

${ }^{1} \mathrm{H}$ NMR Data $\left(400 \mathrm{MHz}, \mathrm{CDCl}_{3}\right)$

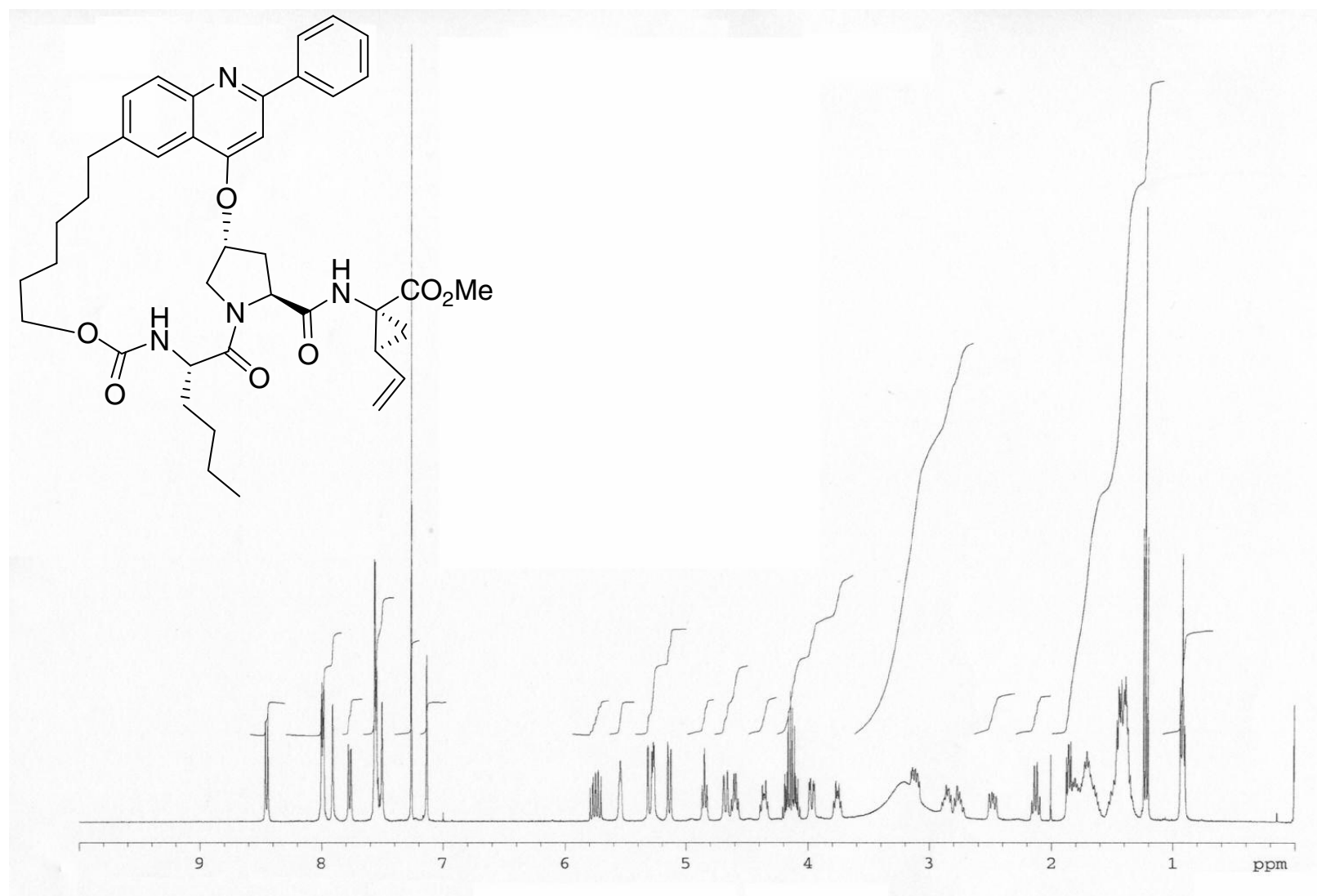

Method A HPLC Data

Method B HPLC Data 
NMR and HPLC Data for Compound 3a

${ }^{1} \mathrm{H}$ NMR Data $\left(400 \mathrm{MHz}, \mathrm{CD}_{3} \mathrm{OD}\right)$

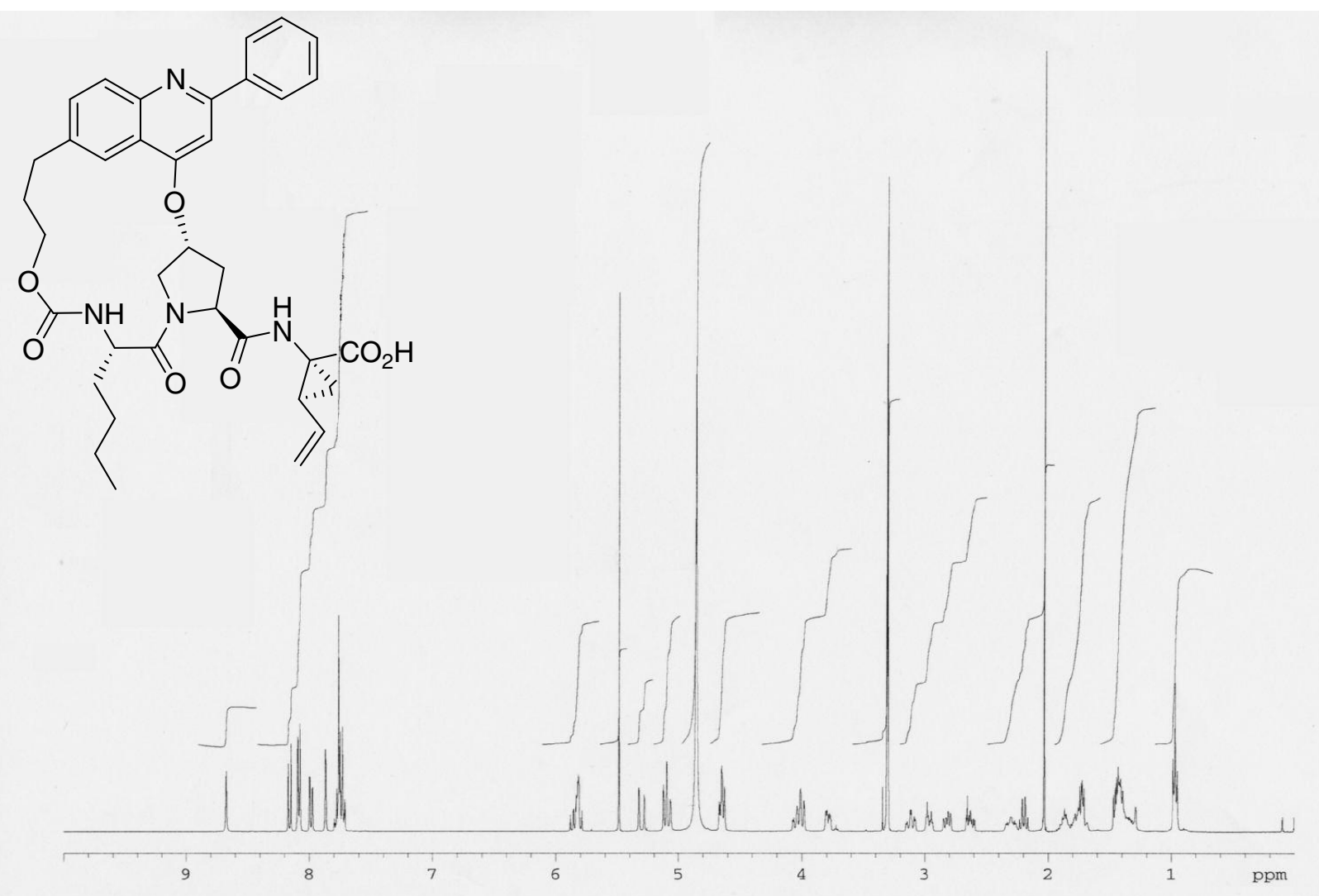

Method A HPLC Data

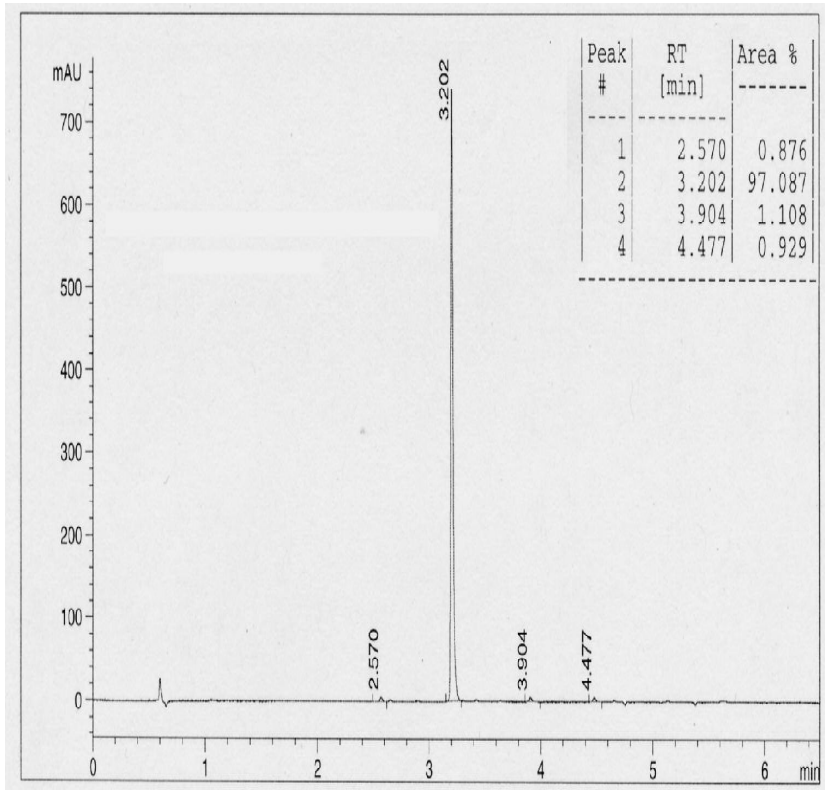

Method B HPLC Data

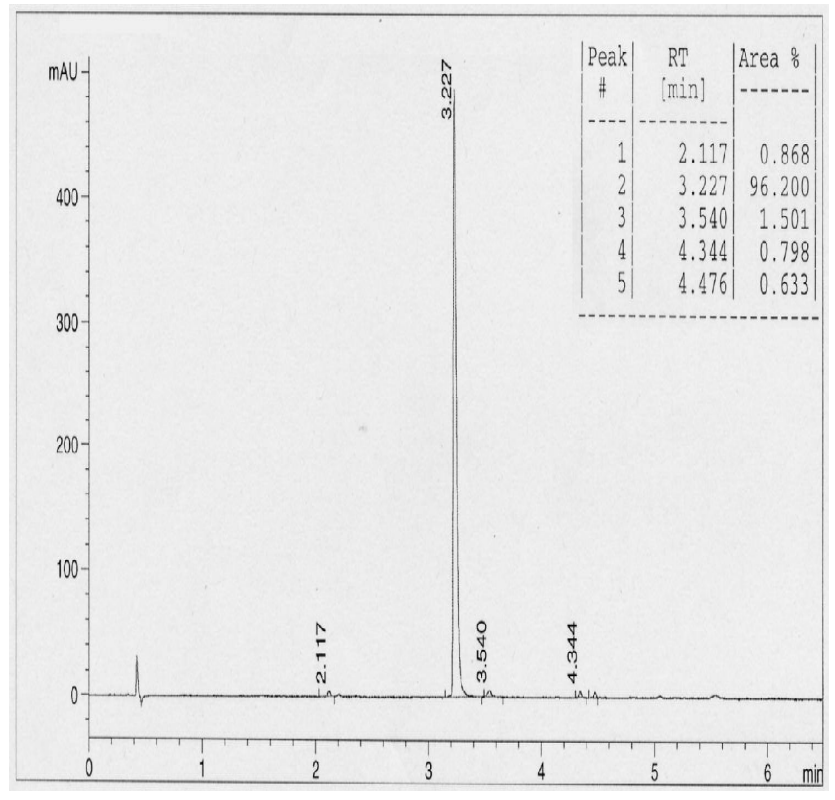


NMR and HPLC Data for Compound 3b

${ }^{1} \mathrm{H}$ NMR Data $\left(400 \mathrm{MHz}, \mathrm{CD}_{3} \mathrm{OD}\right)$

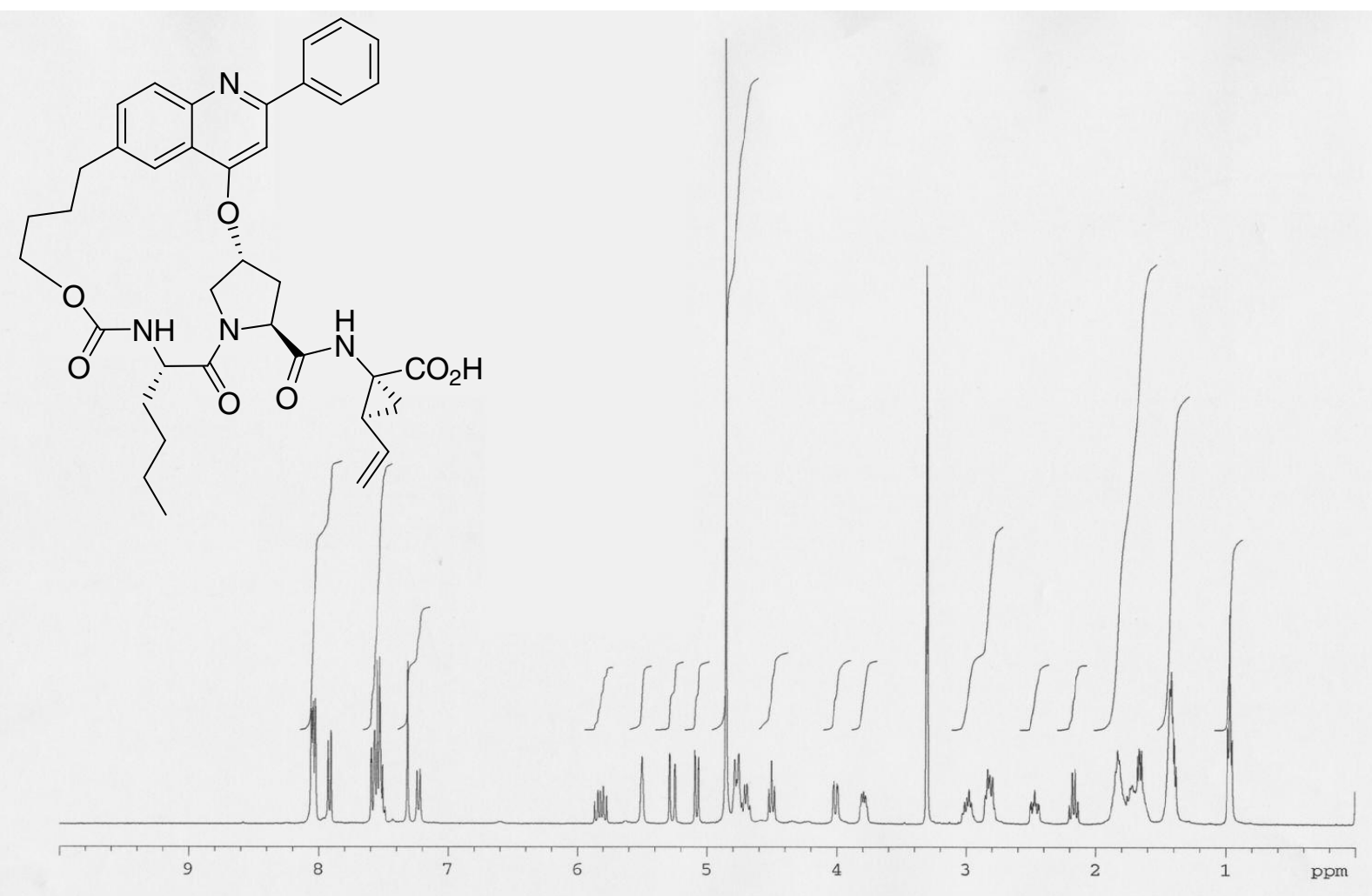

Method A HPLC Data

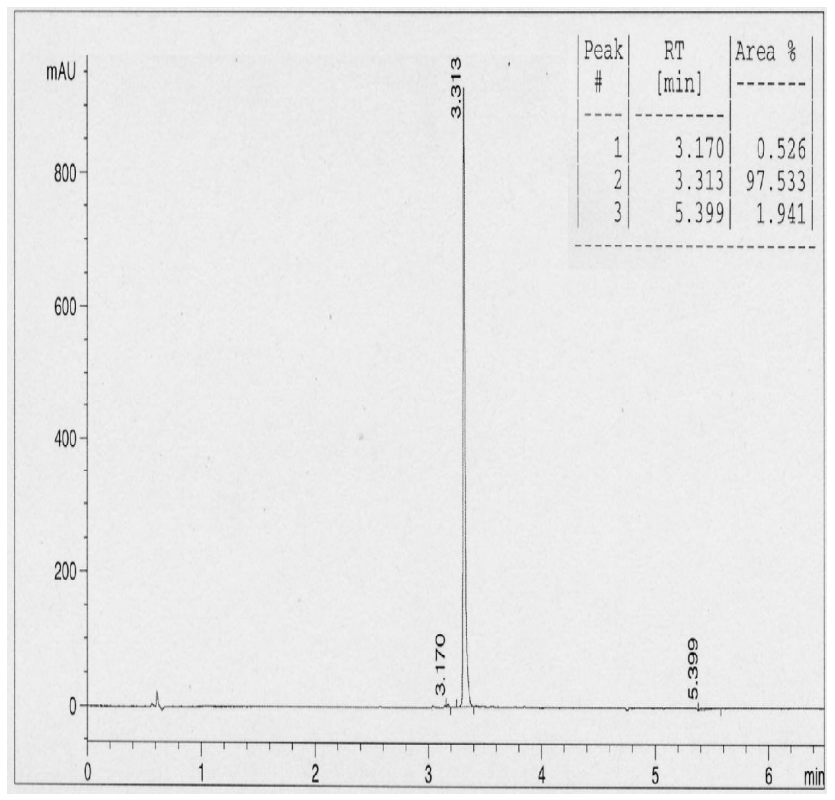

Method B HPLC Data

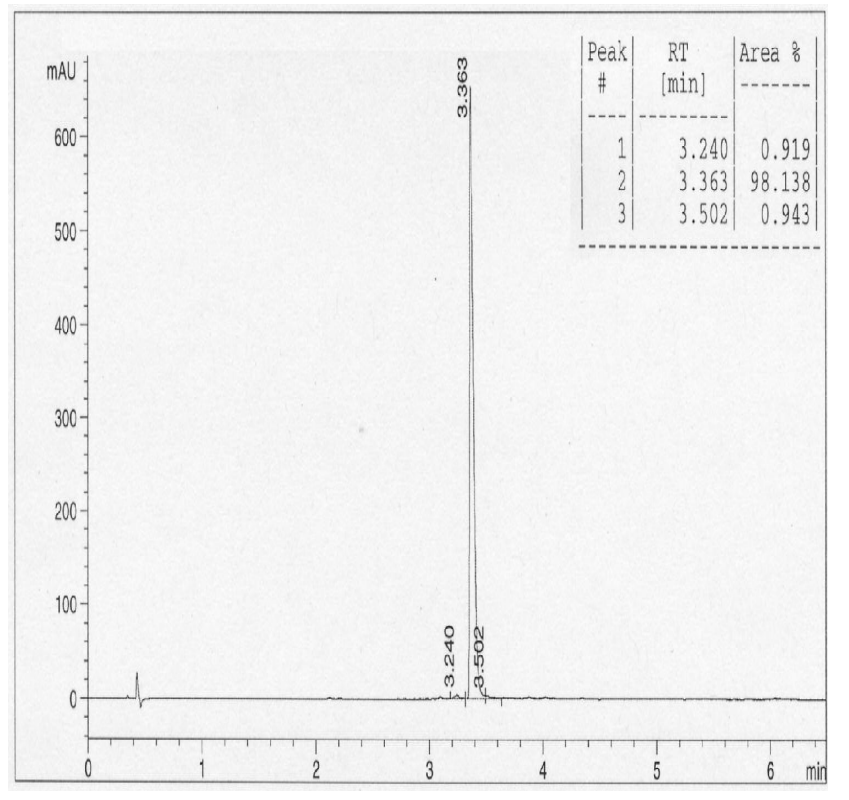


NMR and HPLC Data for Compound 3c

${ }^{1} \mathrm{H}$ NMR Data $\left(500 \mathrm{MHz}, \mathrm{CDCl}_{3}\right)$

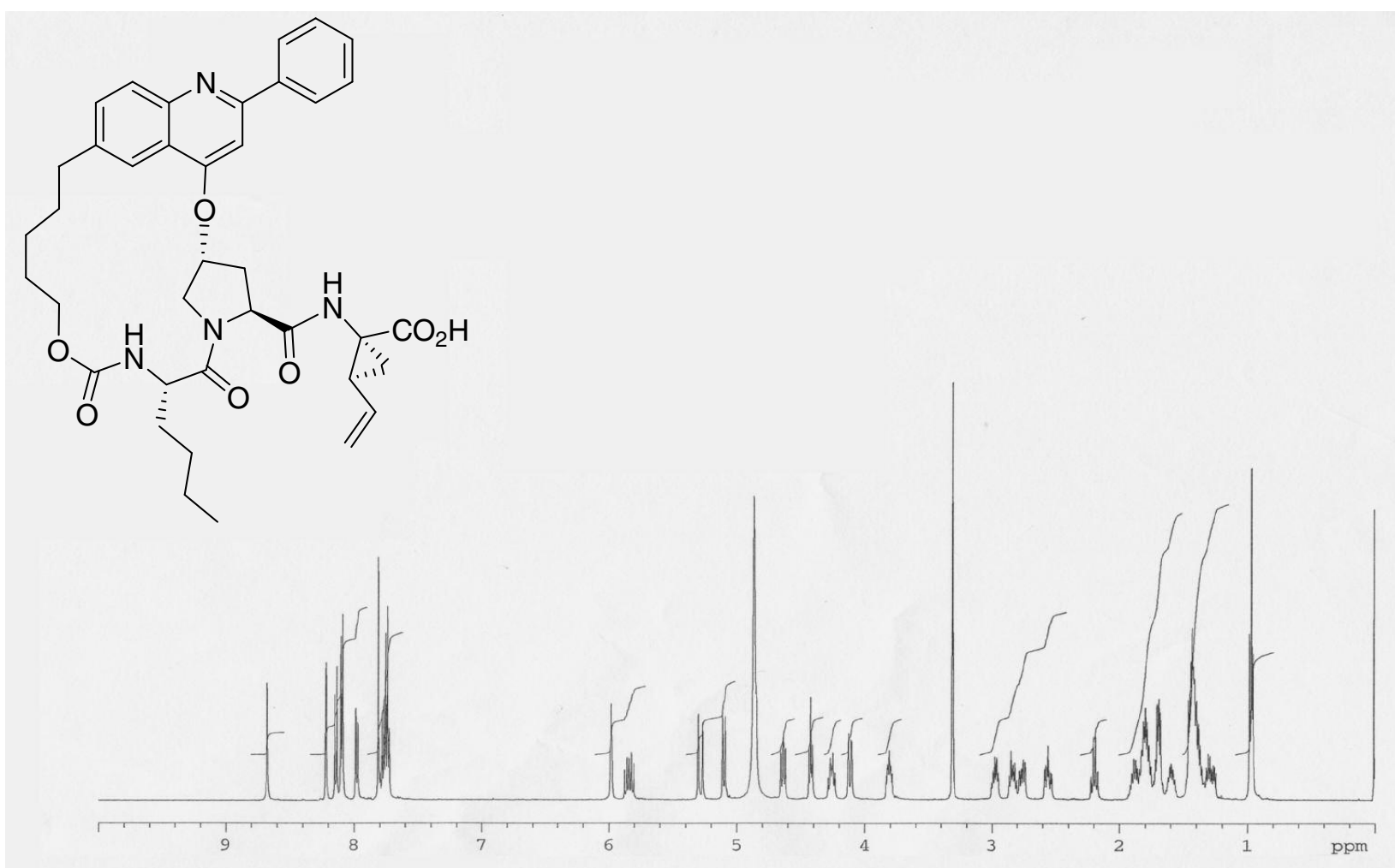

Method A HPLC Data

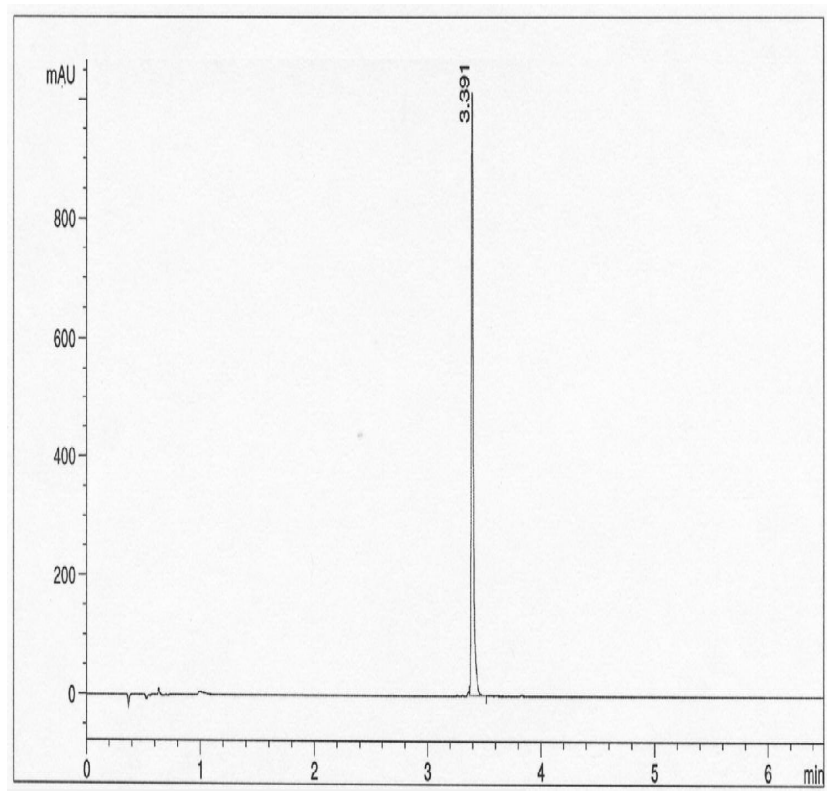

Method B HPLC Data

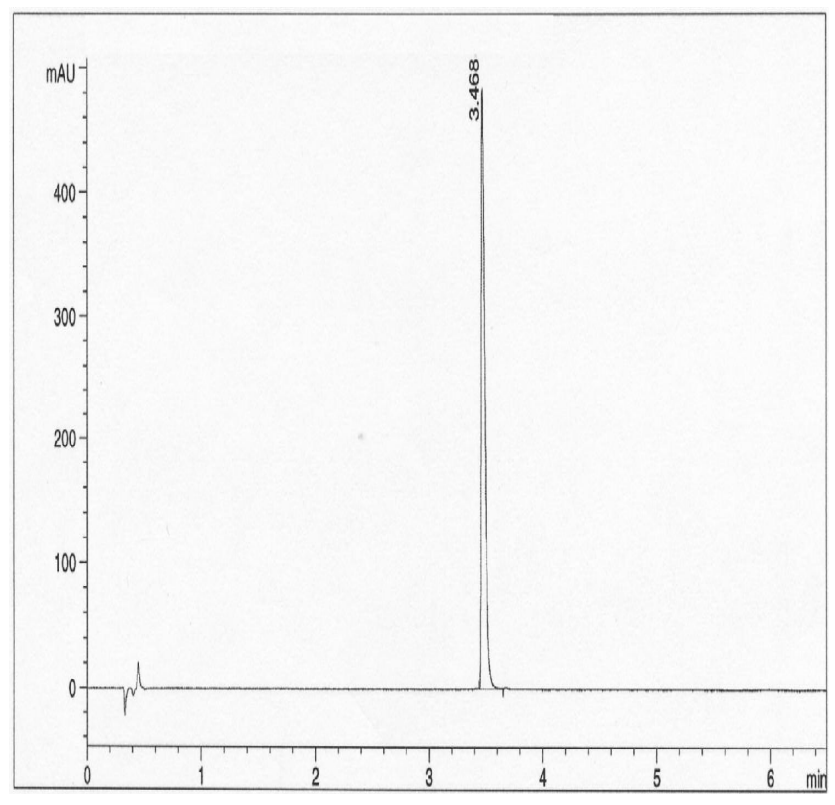




\section{NMR and HPLC Data for Compound 3d}

${ }^{1} \mathrm{H}$ NMR Data $\left(400 \mathrm{MHz}, \mathrm{CD}_{3} \mathrm{OD}\right)$

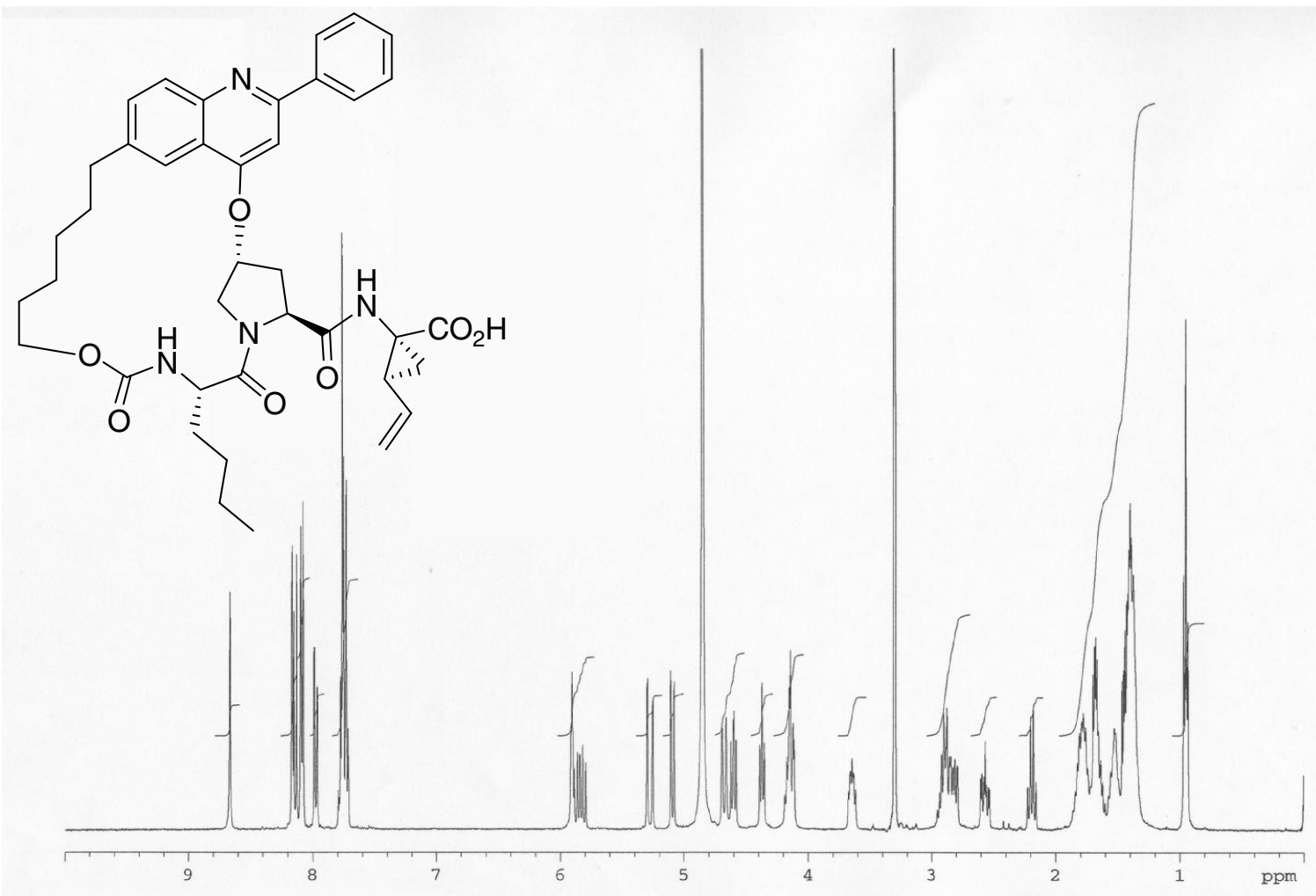

Method A HPLC Data

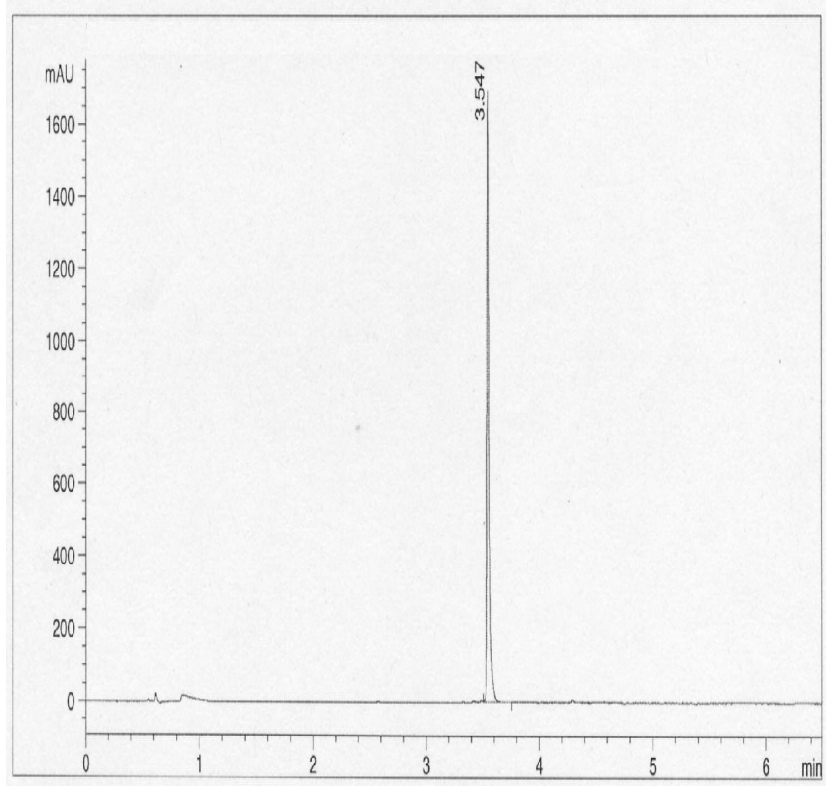

Method B HPLC Data

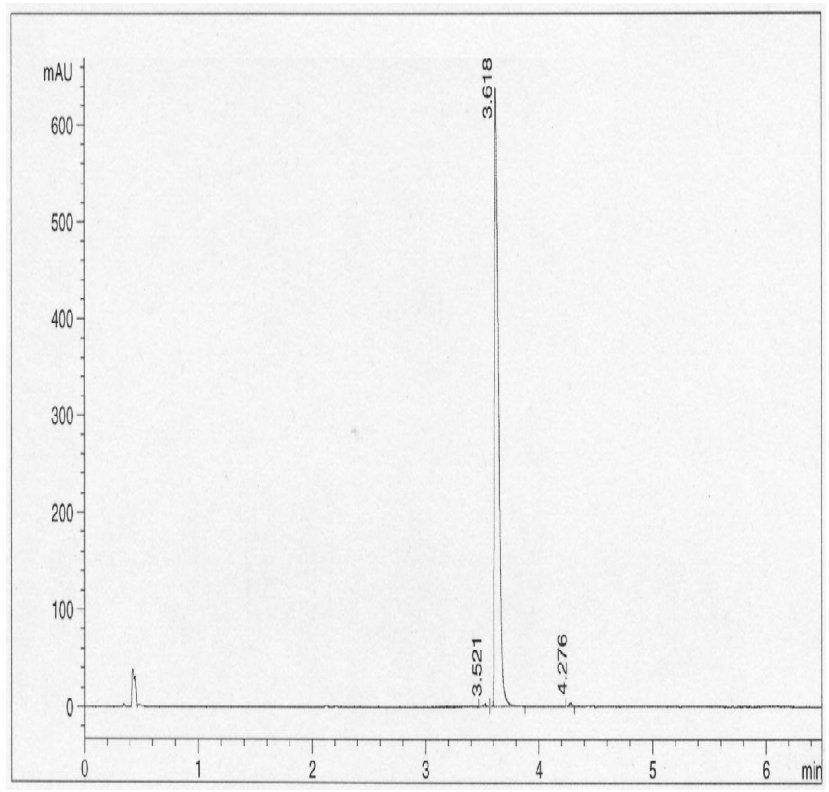


NMR and HPLC Data for Compound 13_1

${ }^{1} \mathrm{H}$ NMR Data (400 MHz, d $\mathrm{d}^{6}$-DMSO)

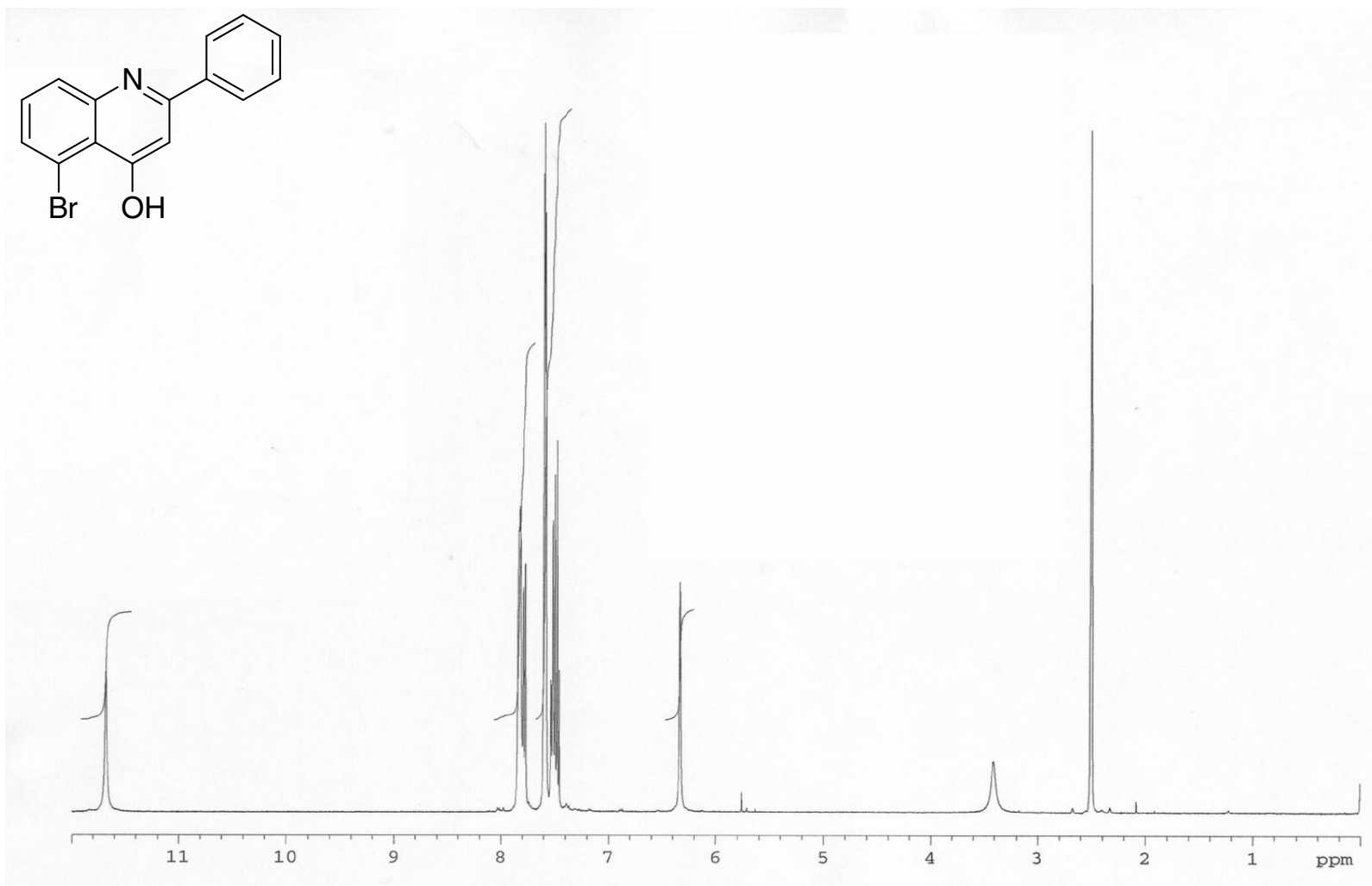

Method A HPLC Data

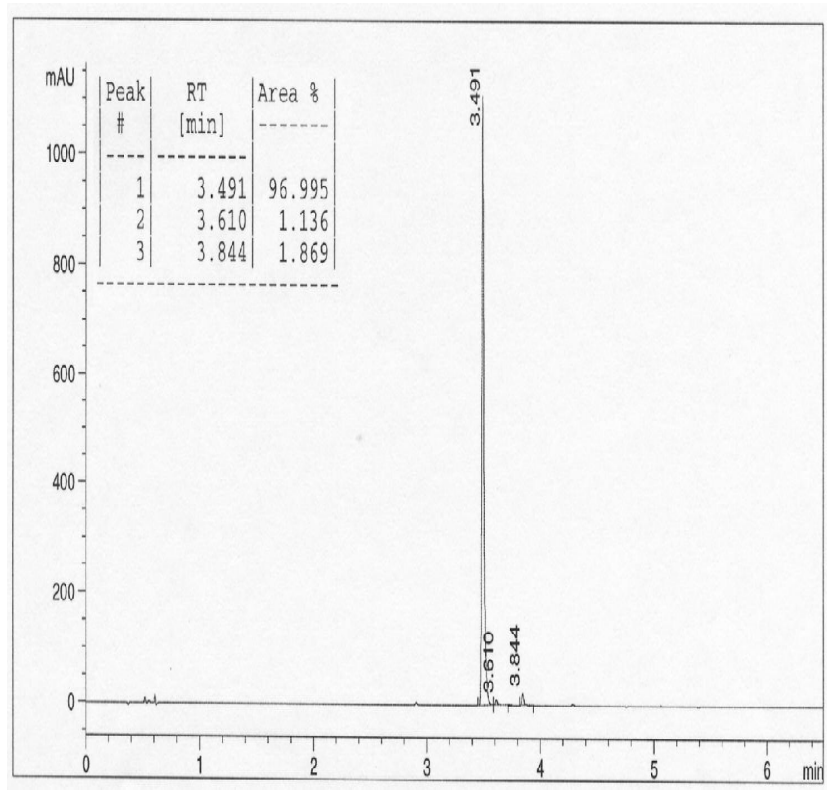

\section{Method B HPLC Data}

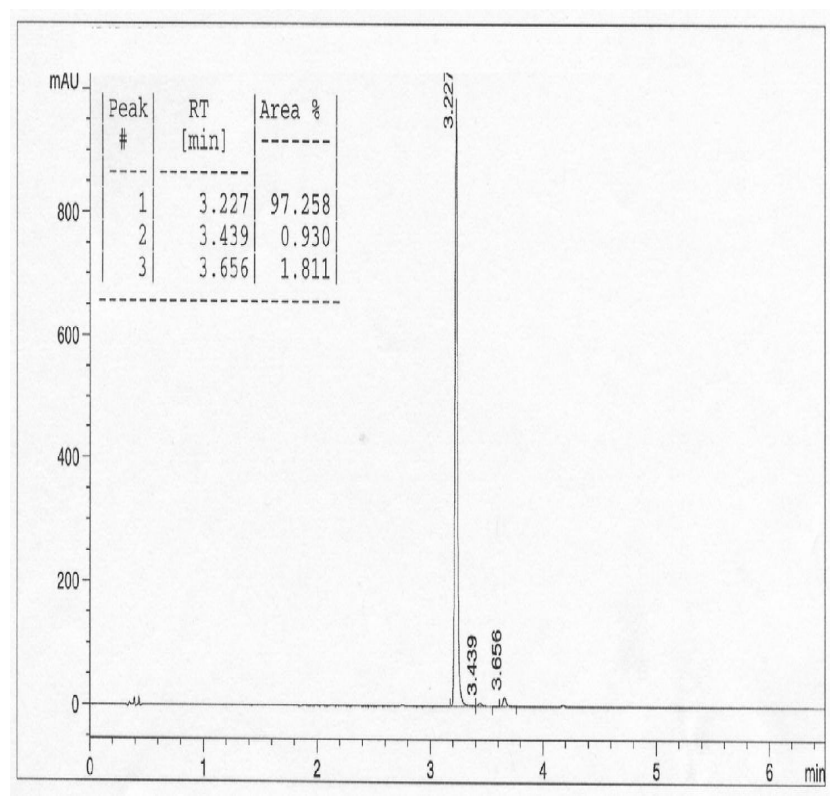


NMR and HPLC Data for Compound 13_2

${ }^{1} \mathrm{H}$ NMR Data $\left(400 \mathrm{MHz}, \mathrm{CD}_{3} \mathrm{OD}\right)$

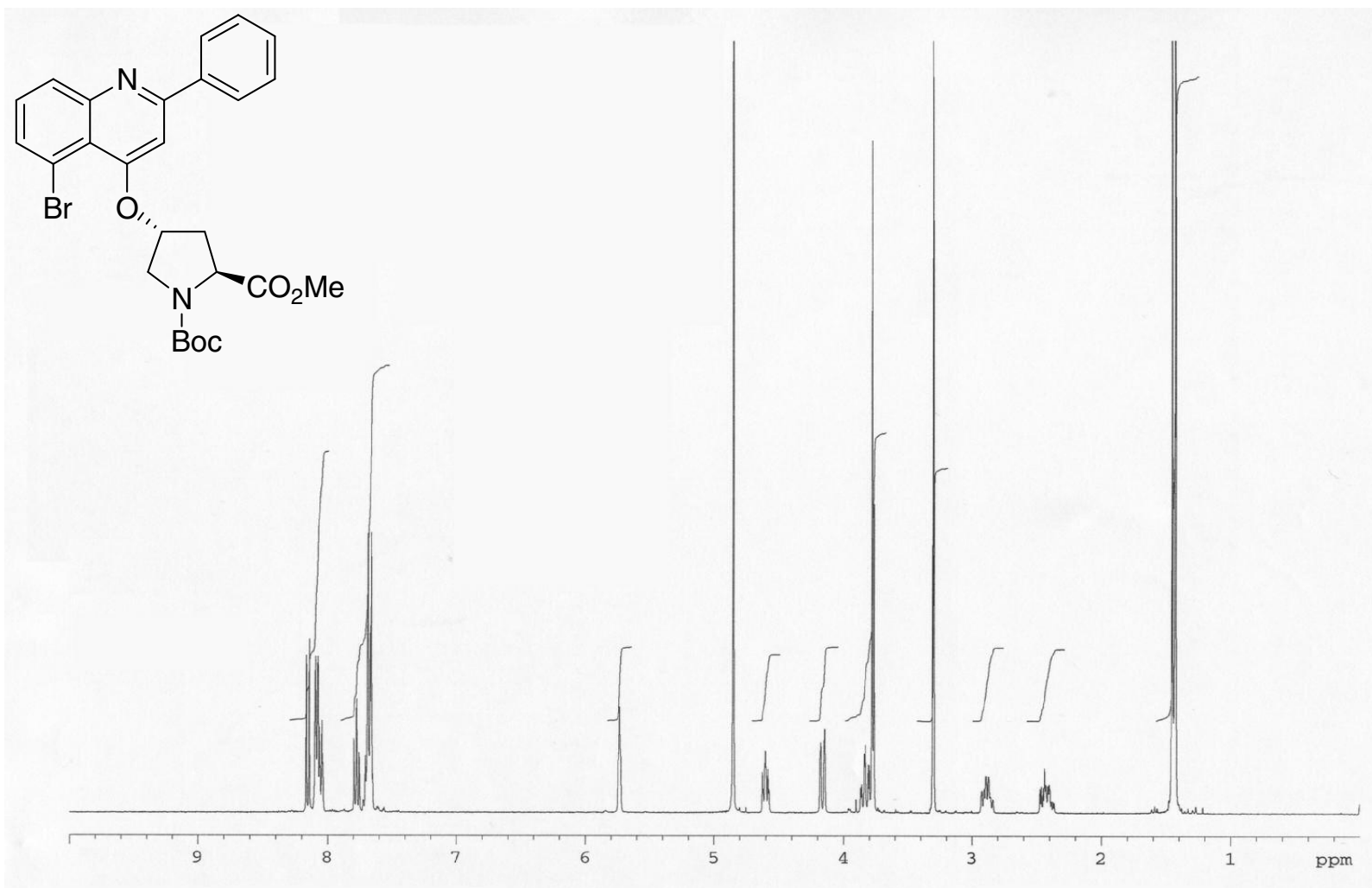

Method A HPLC Data

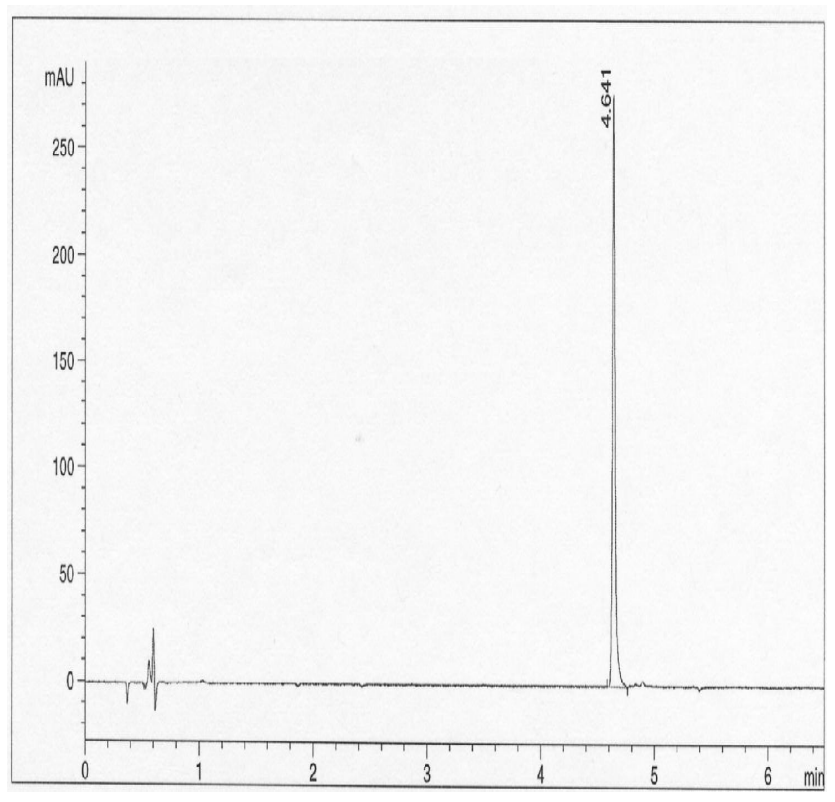

Method B HPLC Data

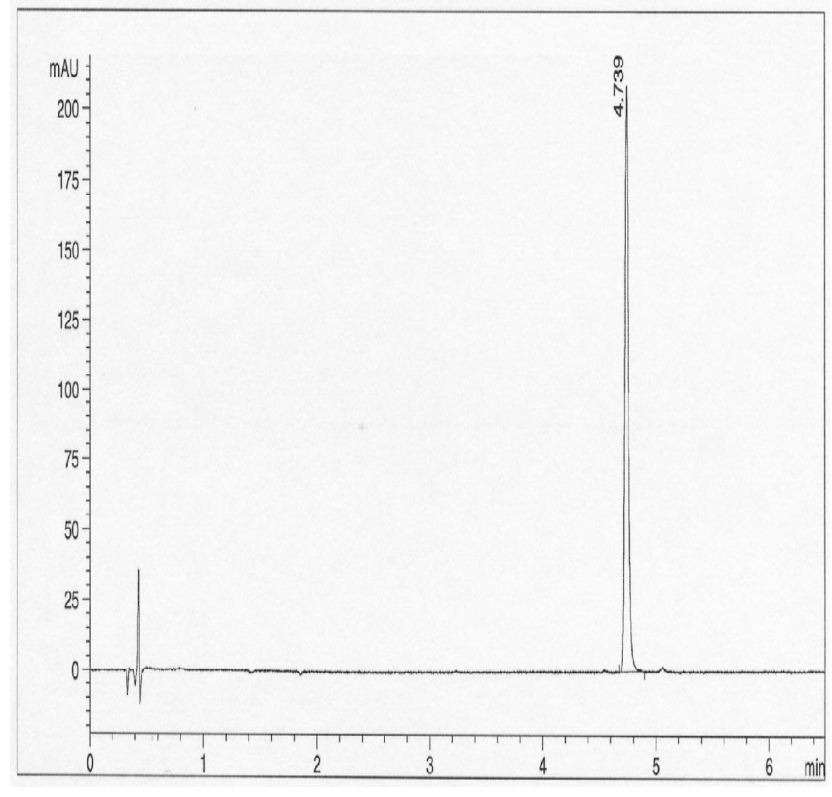


NMR and HPLC Data for Compound 13_3

${ }^{1} \mathrm{H}$ NMR Data $\left(400 \mathrm{MHz}, \mathrm{CD}_{3} \mathrm{OD}\right)$

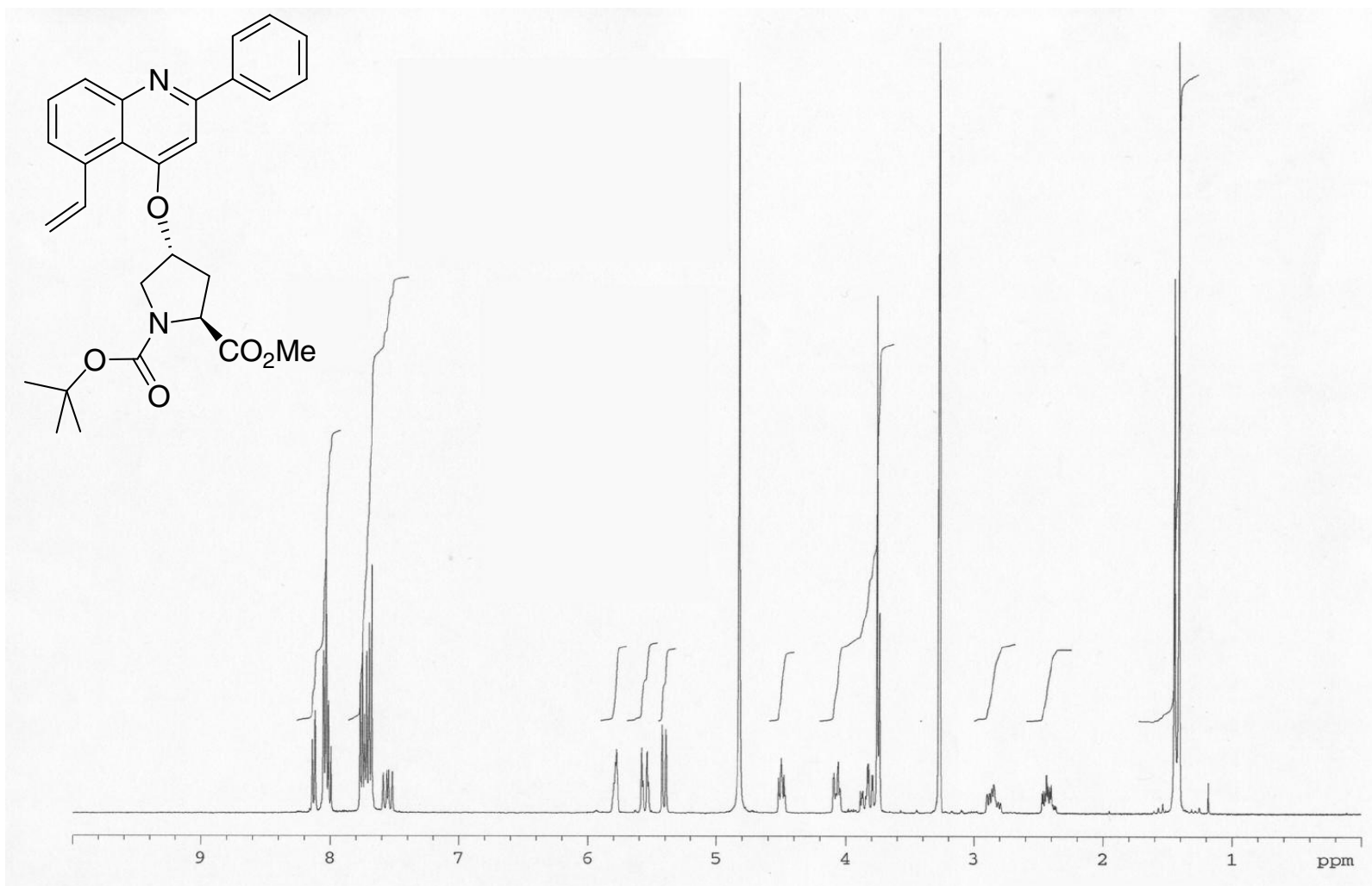

Method A HPLC Data

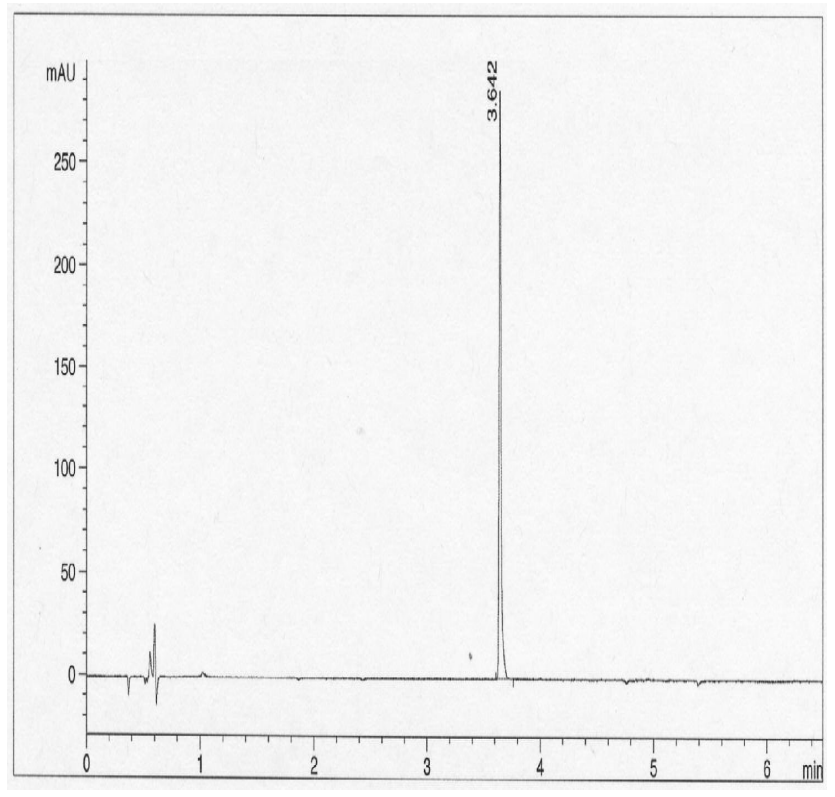

Method B HPLC Data

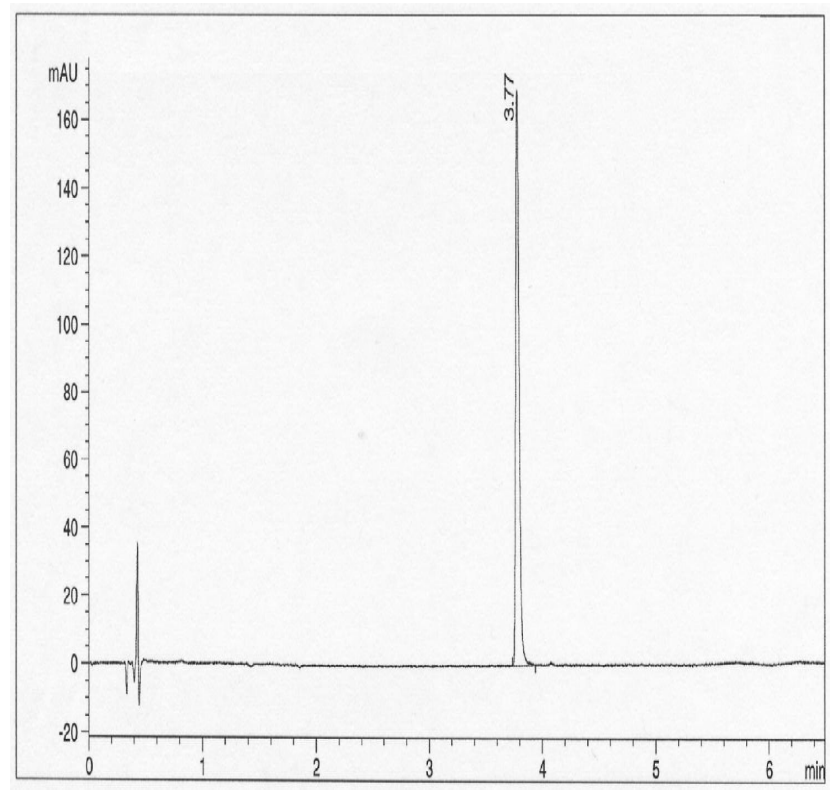


NMR and HPLC Data for Compound 13_4

${ }^{1} \mathrm{H}$ NMR Data $\left(400 \mathrm{MHz}, \mathrm{CDCL}_{3}\right)$

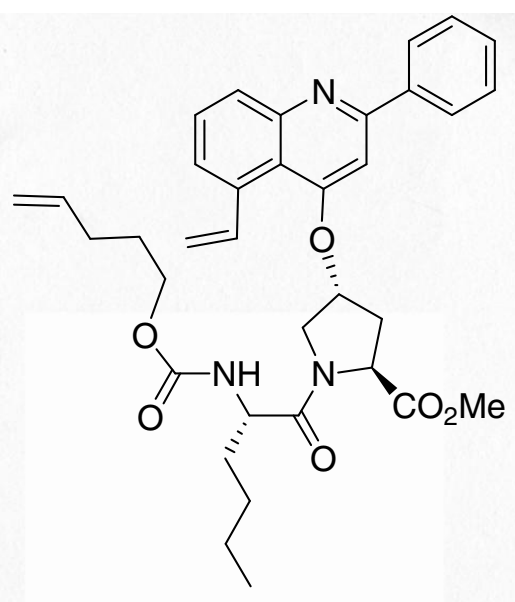

Method A HPLC Data

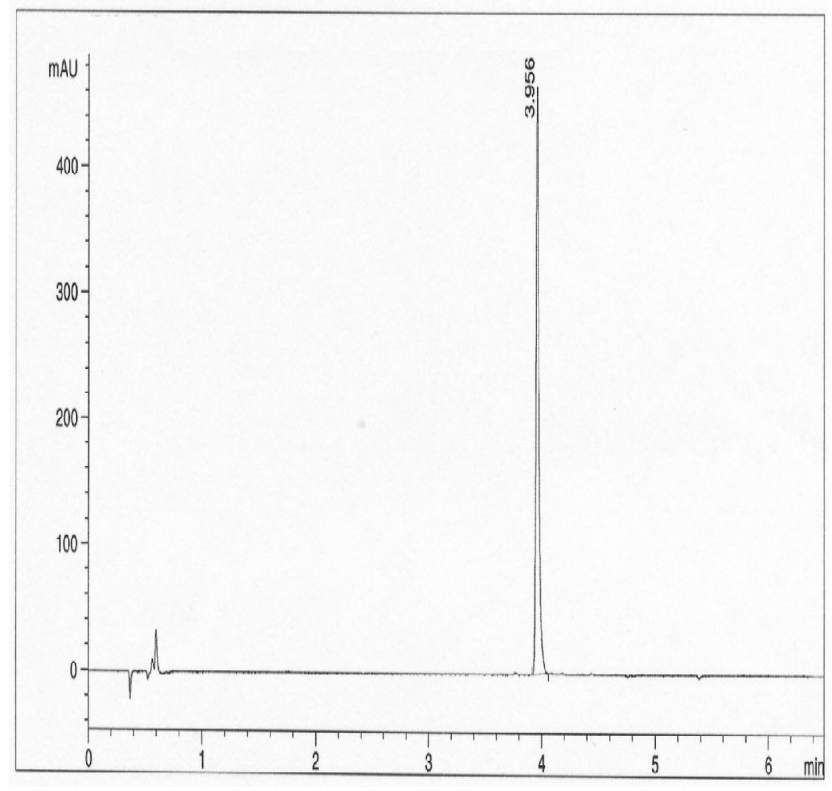

Method B HPLC Data

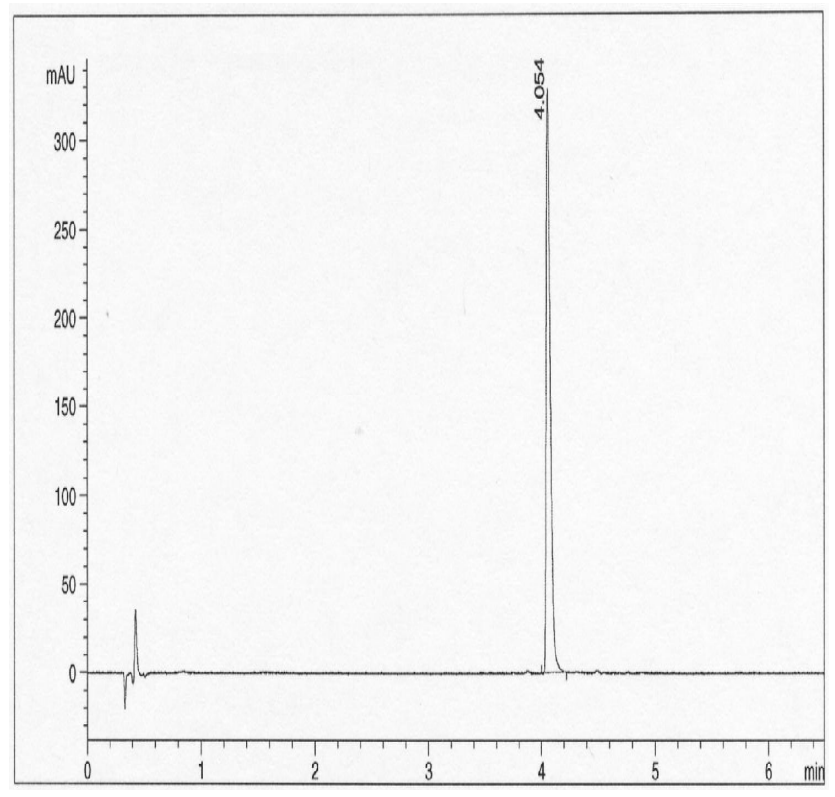


NMR and HPLC Data for Compound 13-5

${ }^{1} \mathrm{H}$ NMR Data $\left(400 \mathrm{MHz}, \mathrm{CDCl}_{3}\right)$

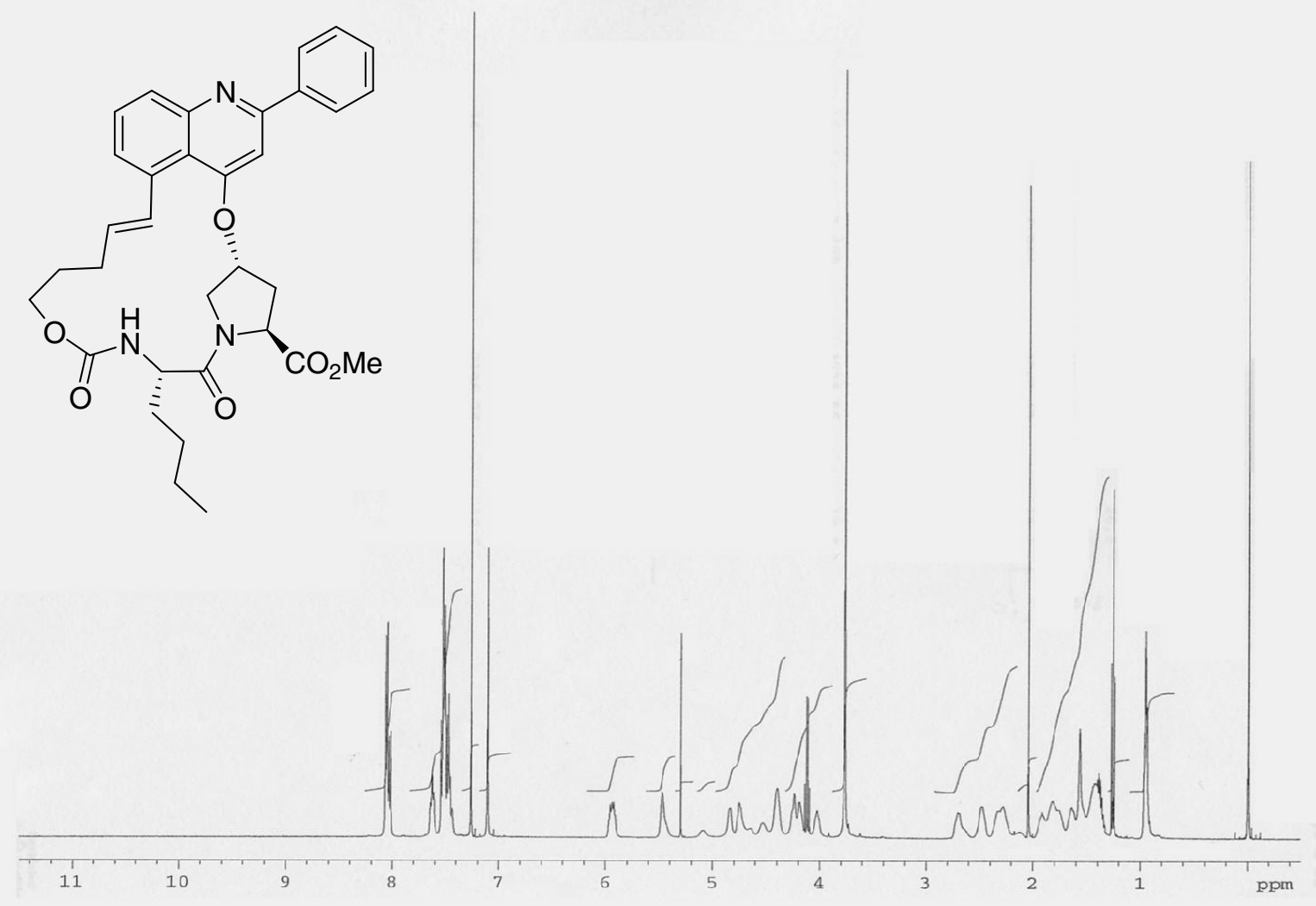

Method A HPLC Data

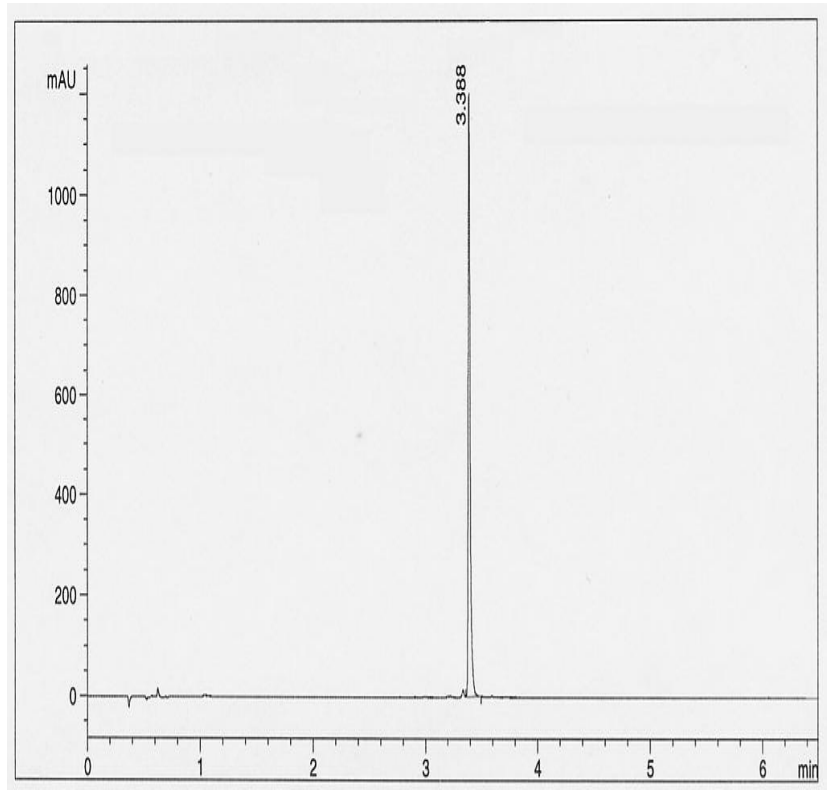

Method B HPLC Data

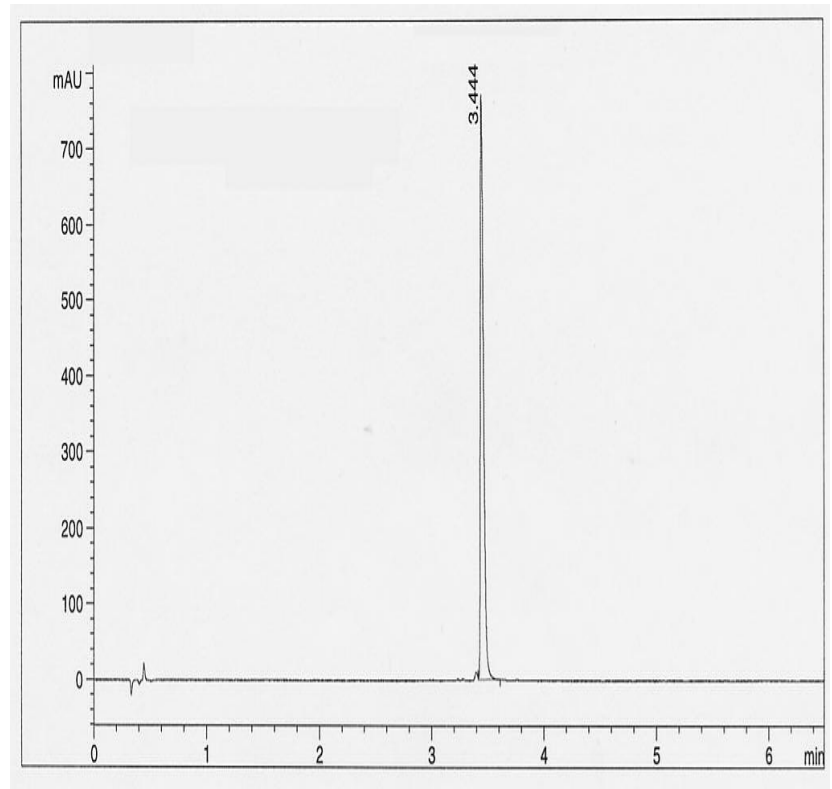


NMR and HPLC Data for Compound 13_6

${ }^{1} \mathrm{H}$ NMR Data (400 MHz, $\mathrm{CDCL}_{3}$ )

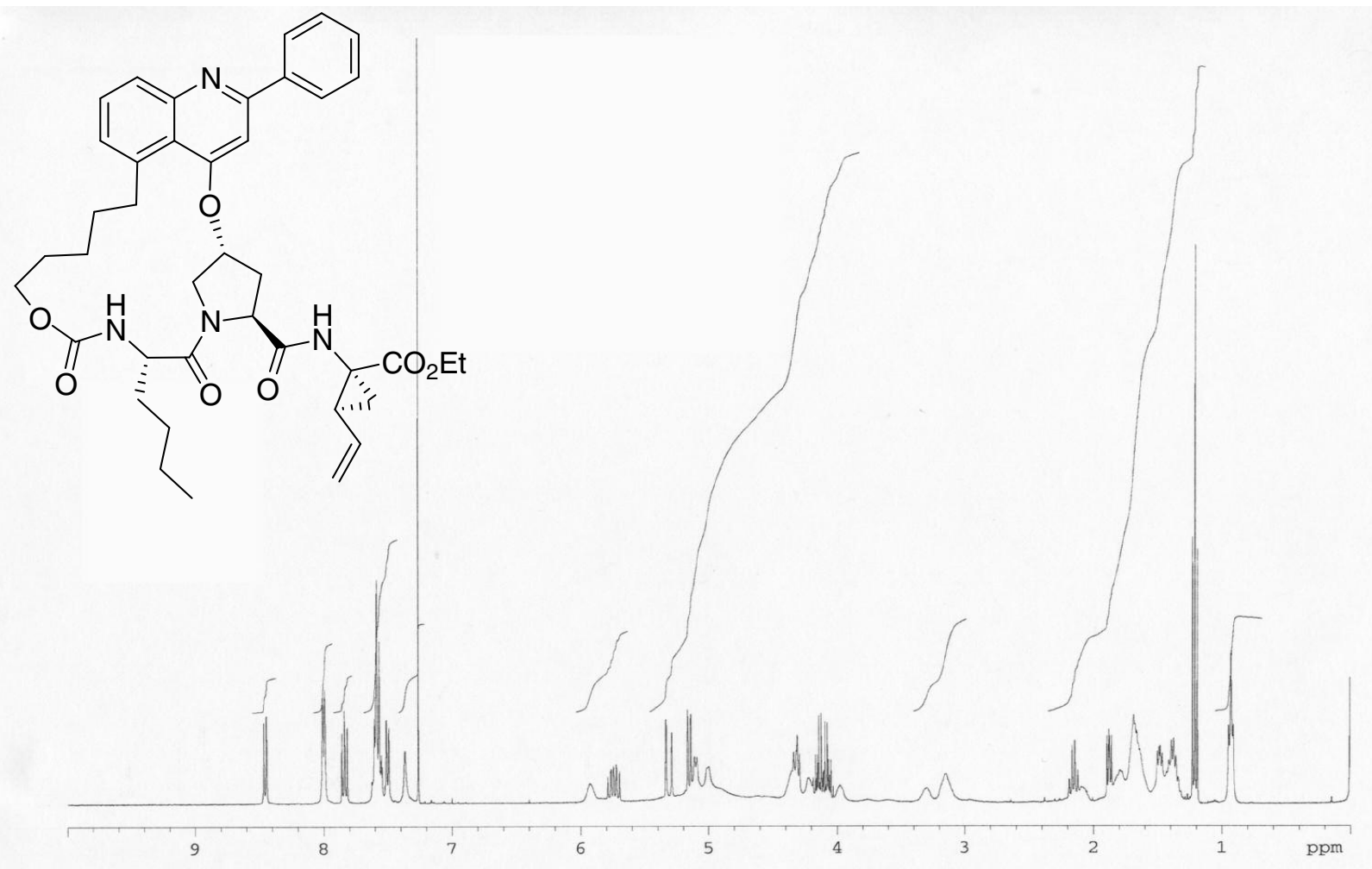

Method A HPLC Data

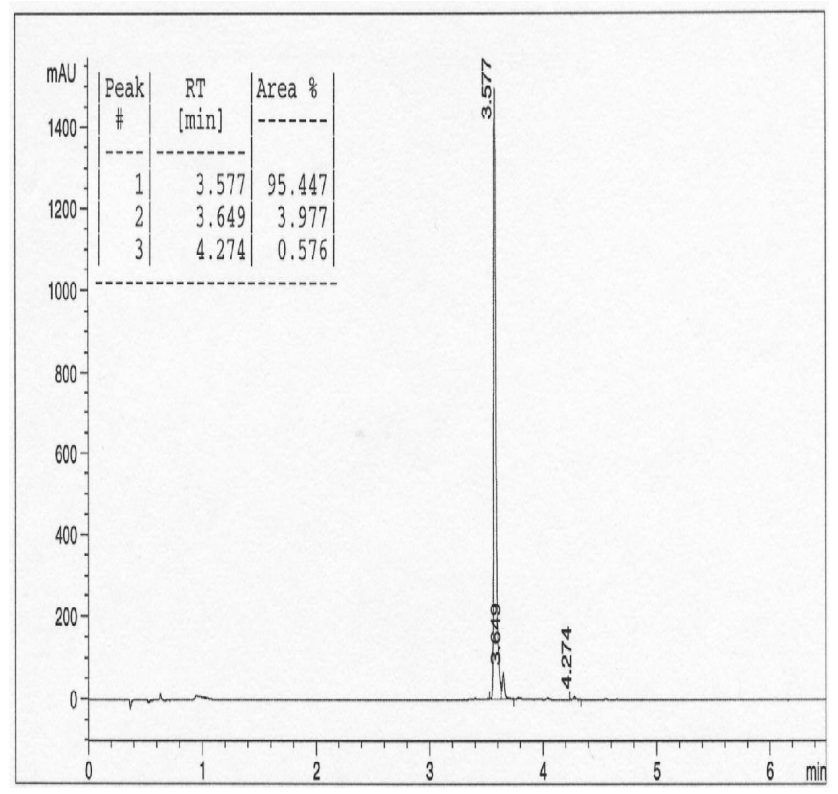

Method B HPLC Data

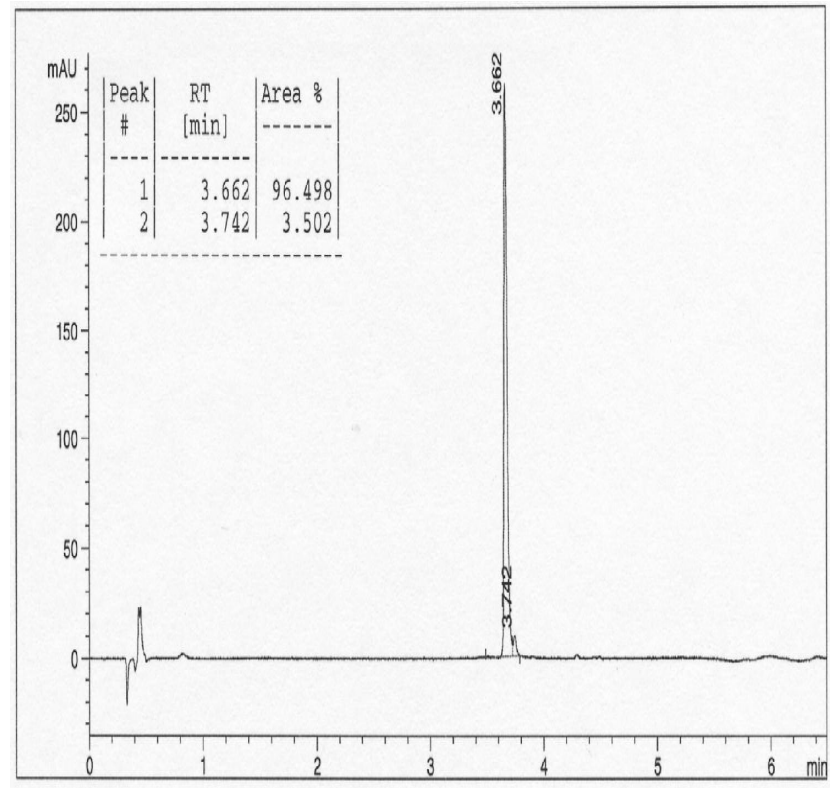




\section{NMR and HPLC Data for Compound 13}

${ }^{1} \mathrm{H}$ NMR Data $\left(400 \mathrm{MHz}, \mathrm{CD}_{3} \mathrm{OD}\right)$

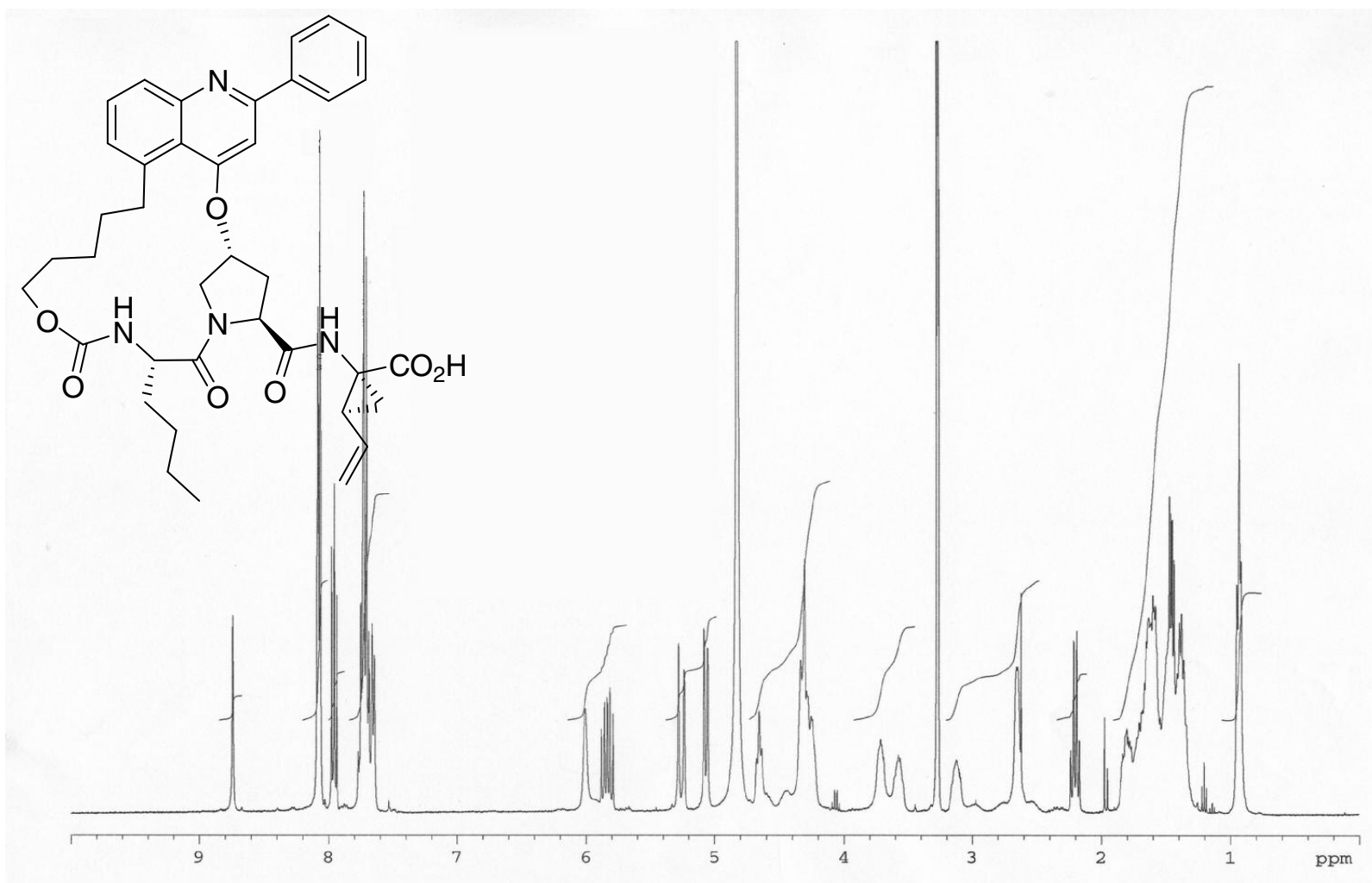

Method A HPLC Data

Method B HPLC Data 
NMR and HPLC Data for Compound 14_1

${ }^{1} \mathrm{H}$ NMR Data $\left(400 \mathrm{MHz}, \mathrm{CDCl}_{3}\right)$

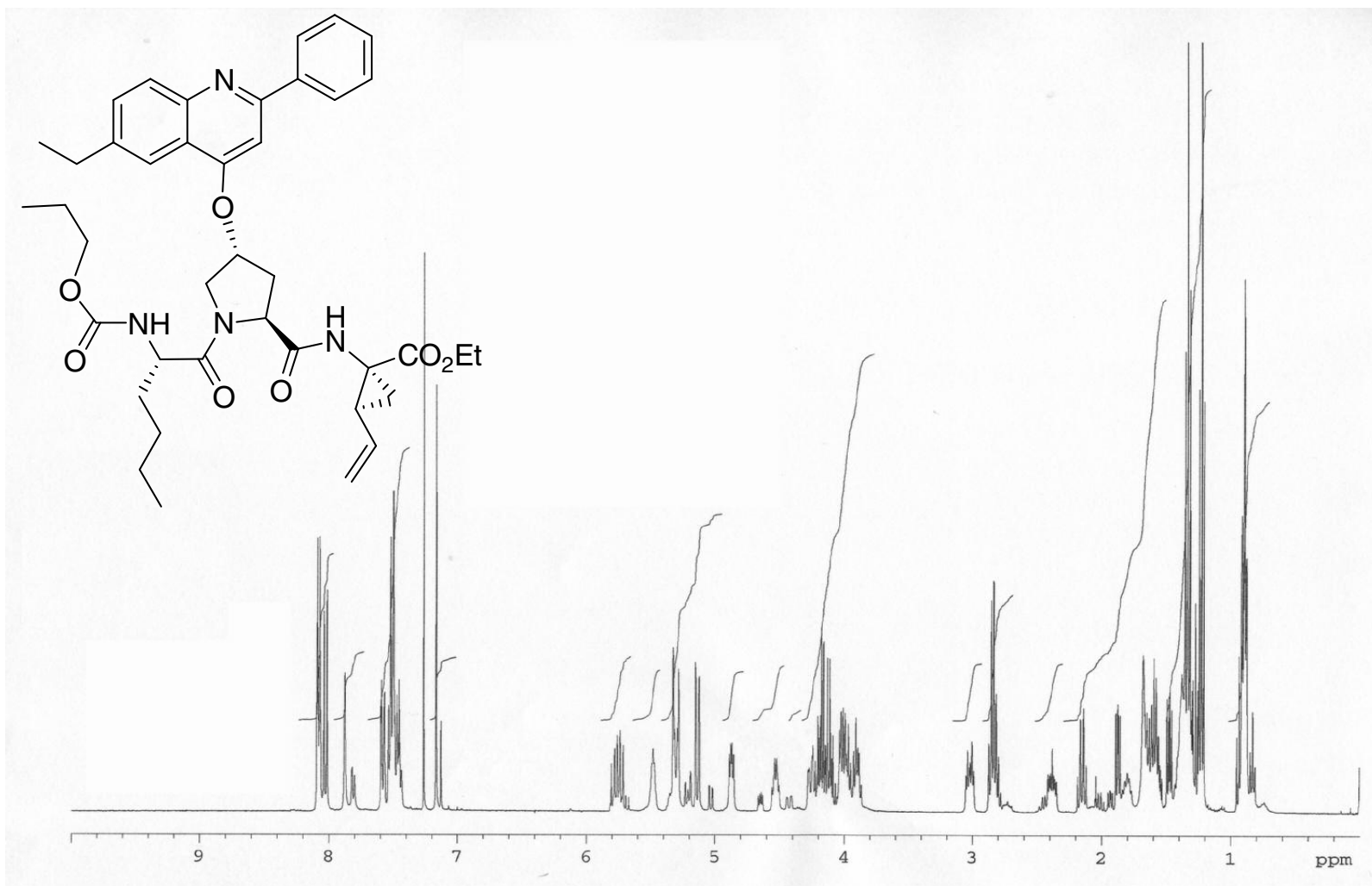

Method A HPLC Data

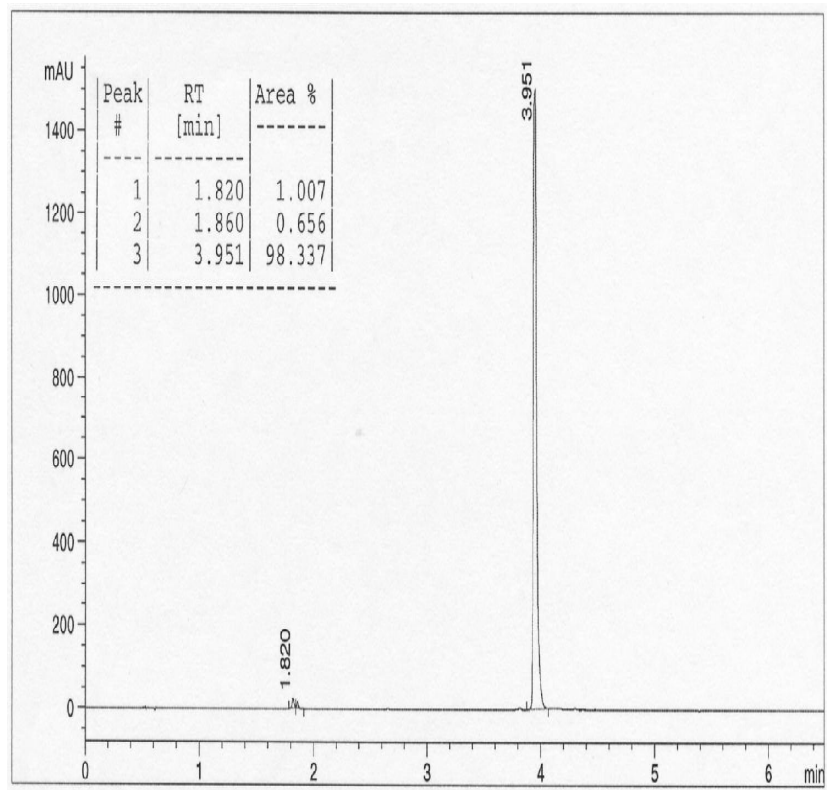

Method B HPLC Data

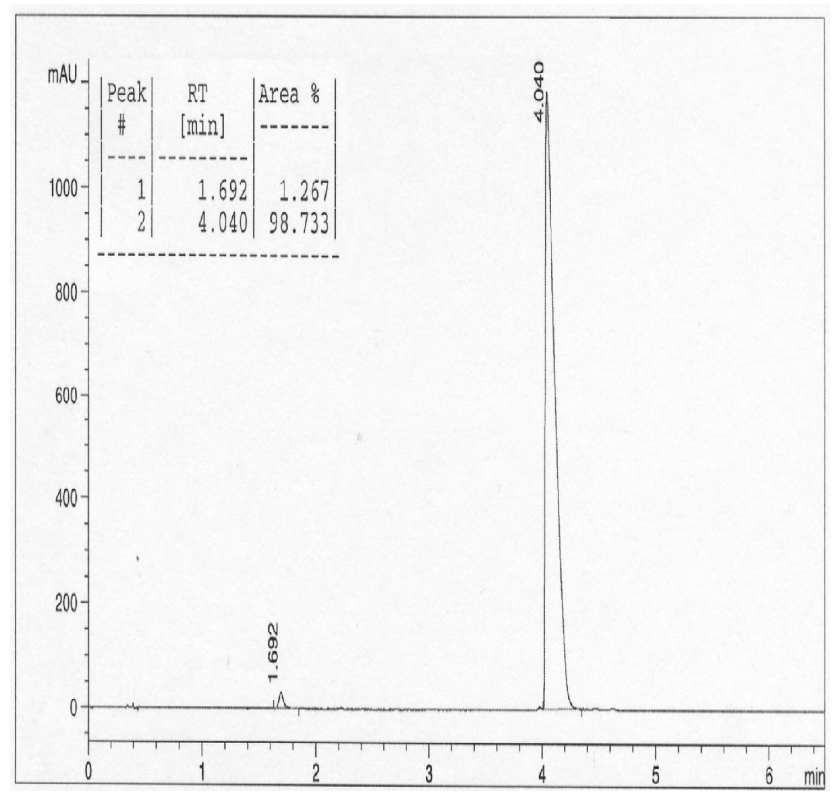


NMR and HPLC Data for Compound 14

${ }^{1} \mathrm{H}$ NMR Data $\left(400 \mathrm{MHz}, \mathrm{CD}_{3} \mathrm{OD}\right)$

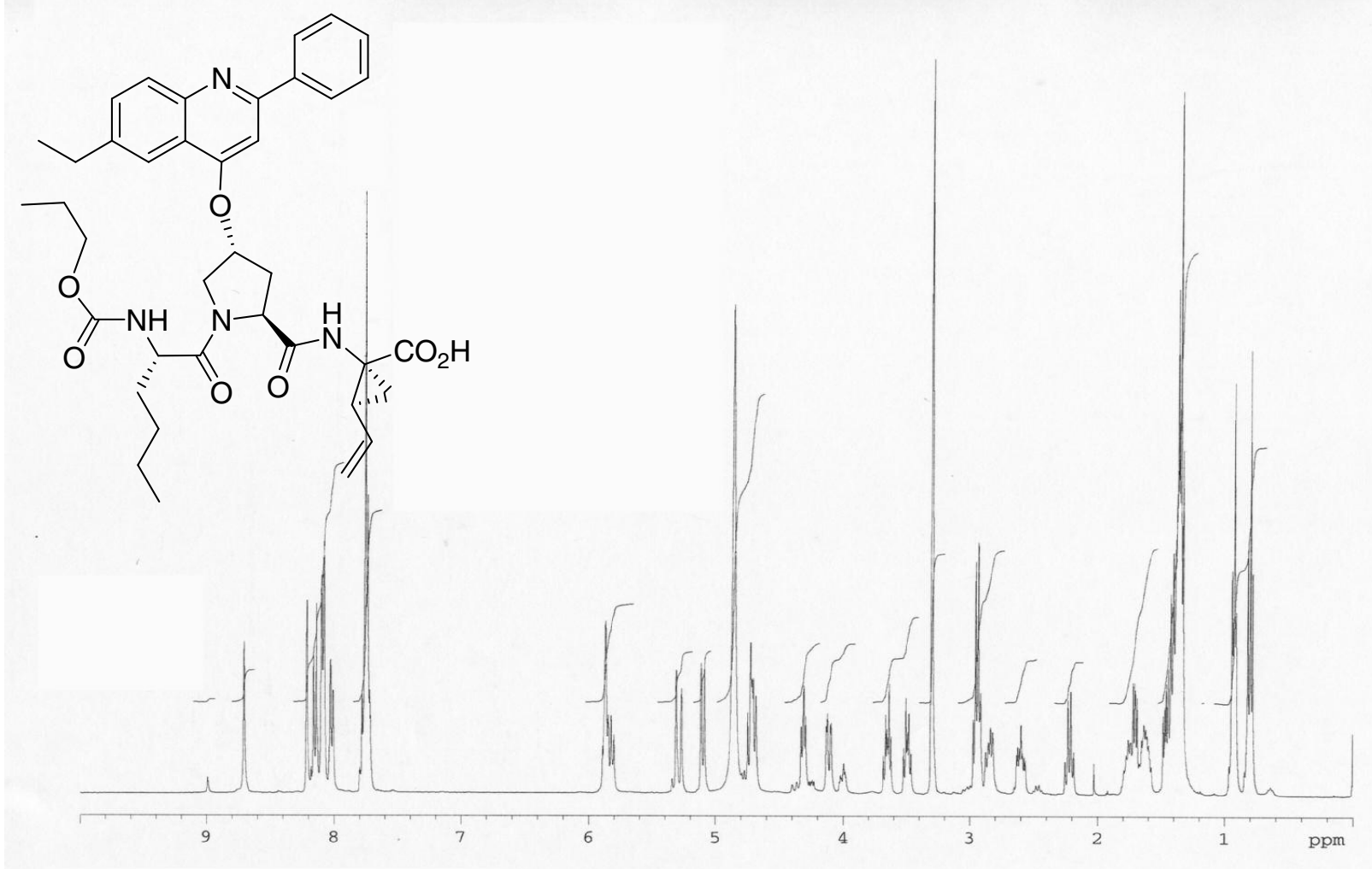

Method A HPLC Data

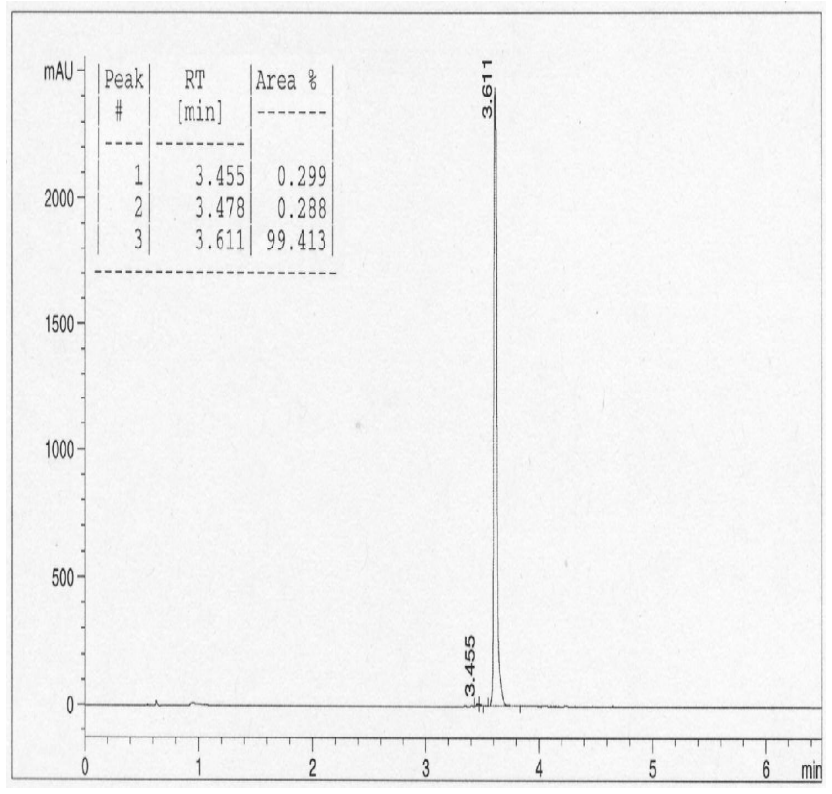

Method B HPLC Data

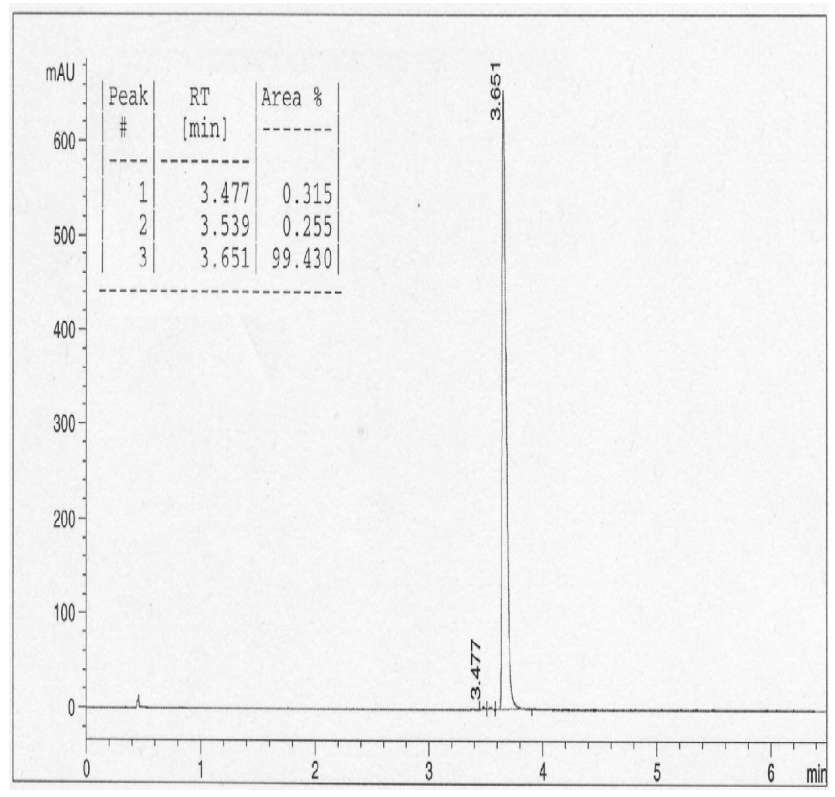




\section{NMR and HPLC Data for Compound 15}

${ }^{1} \mathrm{H}$ NMR Data $\left(500 \mathrm{MHz}, \mathrm{CD}_{3} \mathrm{OD}\right)$

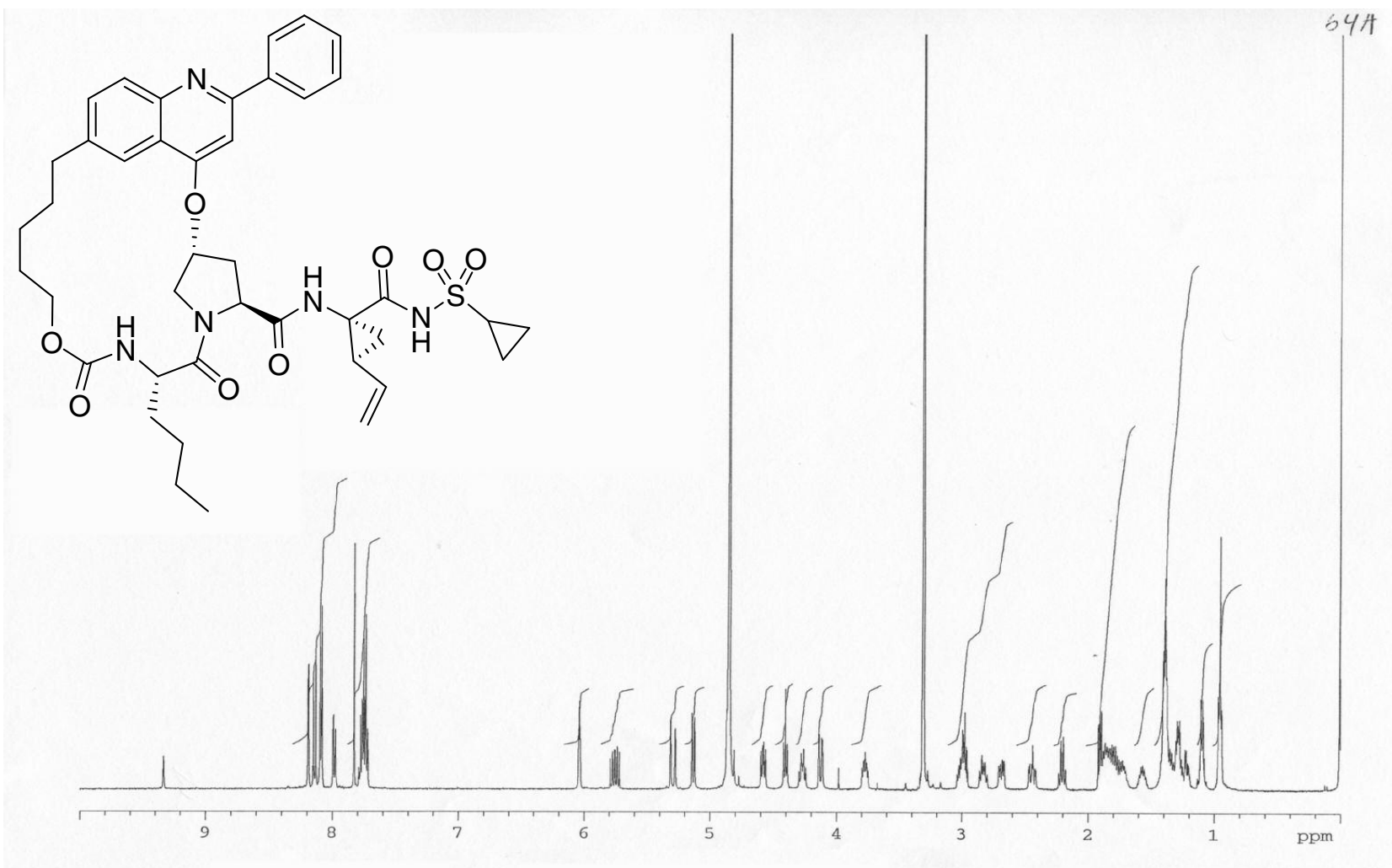

Method A HPLC Data

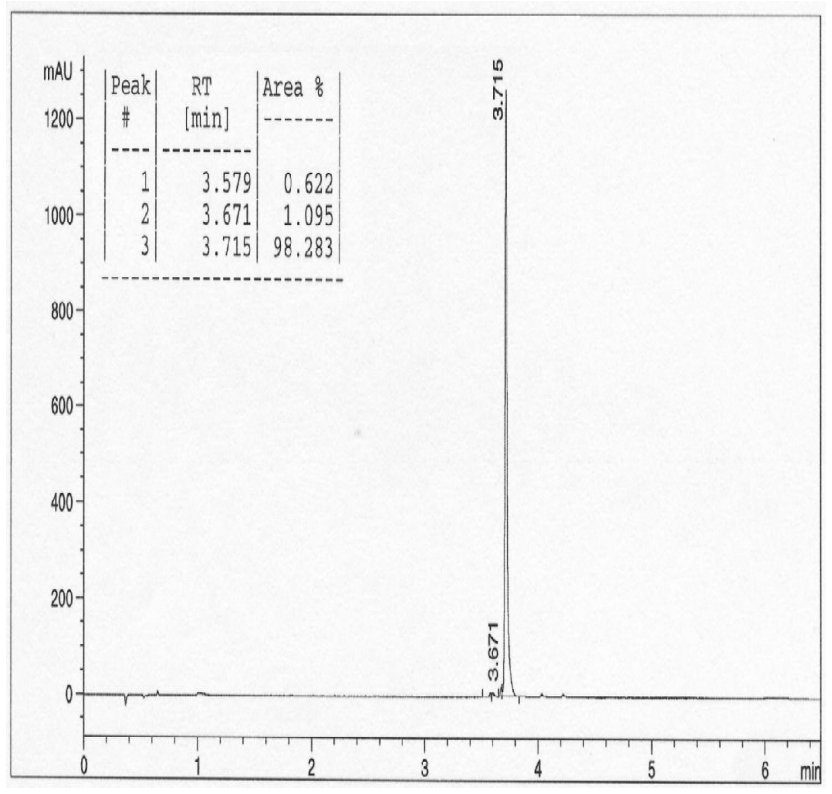

Method B HPLC Data

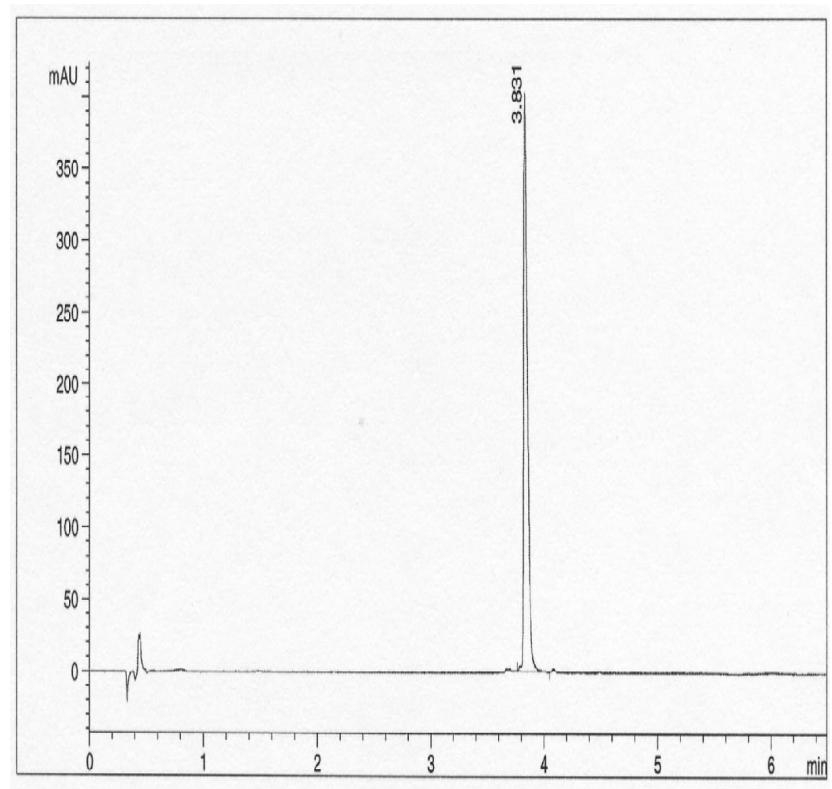


NMR and HPLC Data for Compound 16_1

${ }^{1} \mathrm{H}$ NMR Data $\left(400 \mathrm{MHz}, \mathrm{CDCl}_{3}\right)$

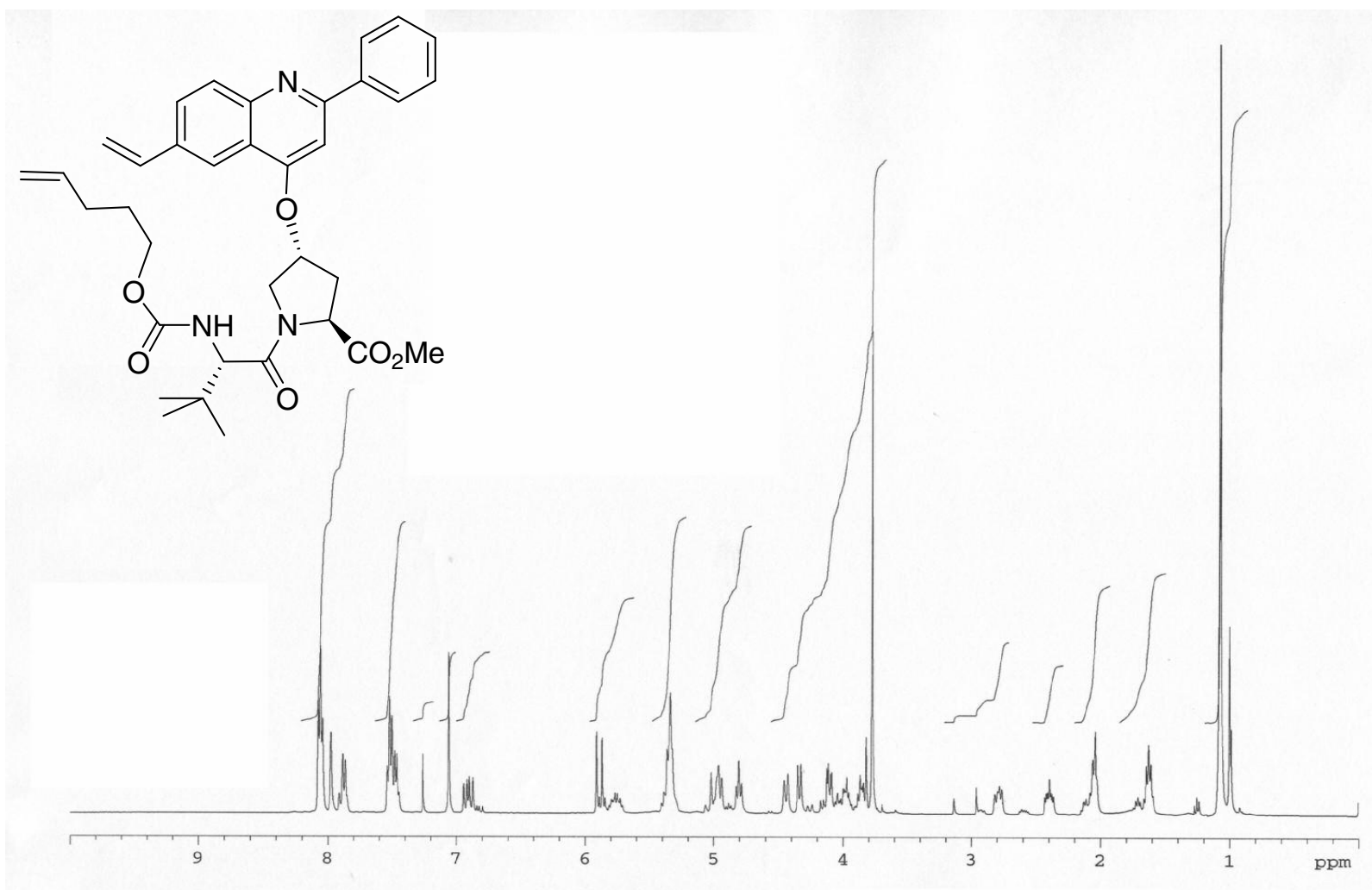

Method A HPLC Data

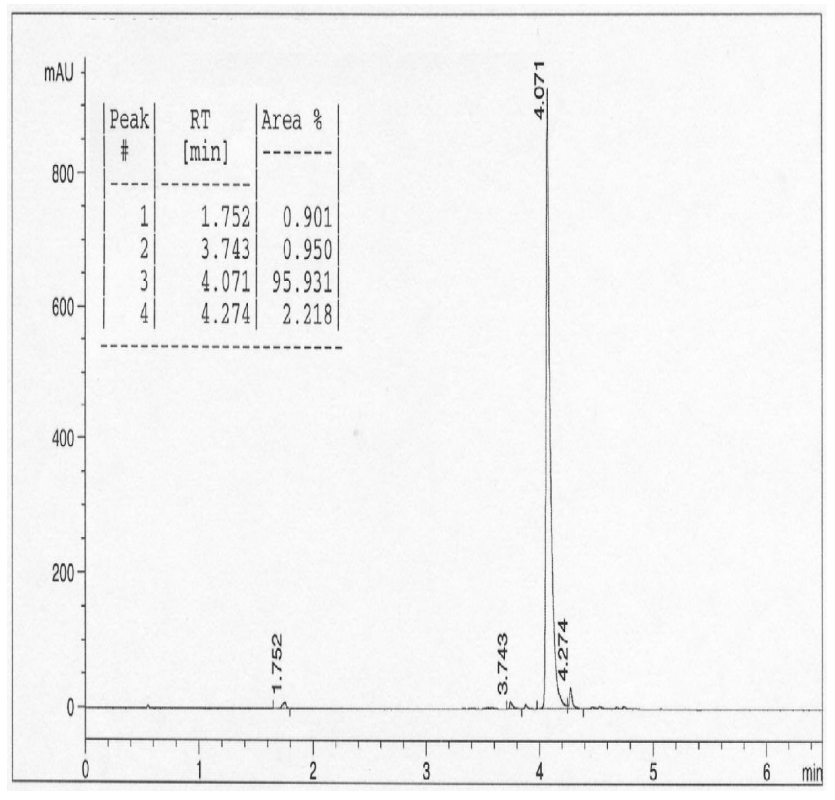

Method B HPLC Data

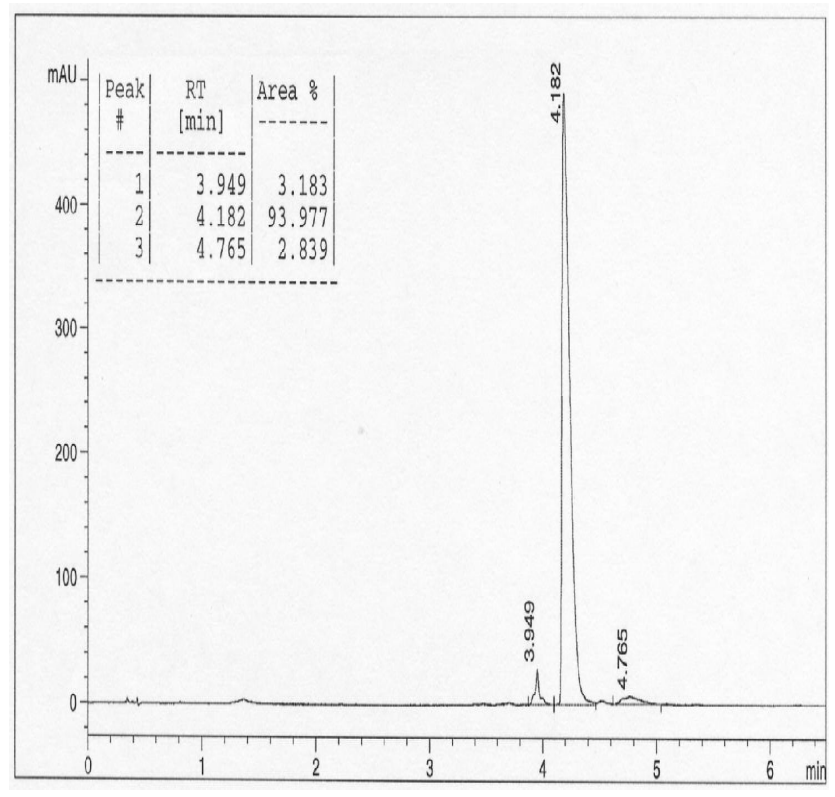




\section{NMR and HPLC Data for Compound 16_2}

${ }^{1} \mathrm{H}$ NMR Data $\left(400 \mathrm{MHz}, \mathrm{CDCl}_{3}\right)$

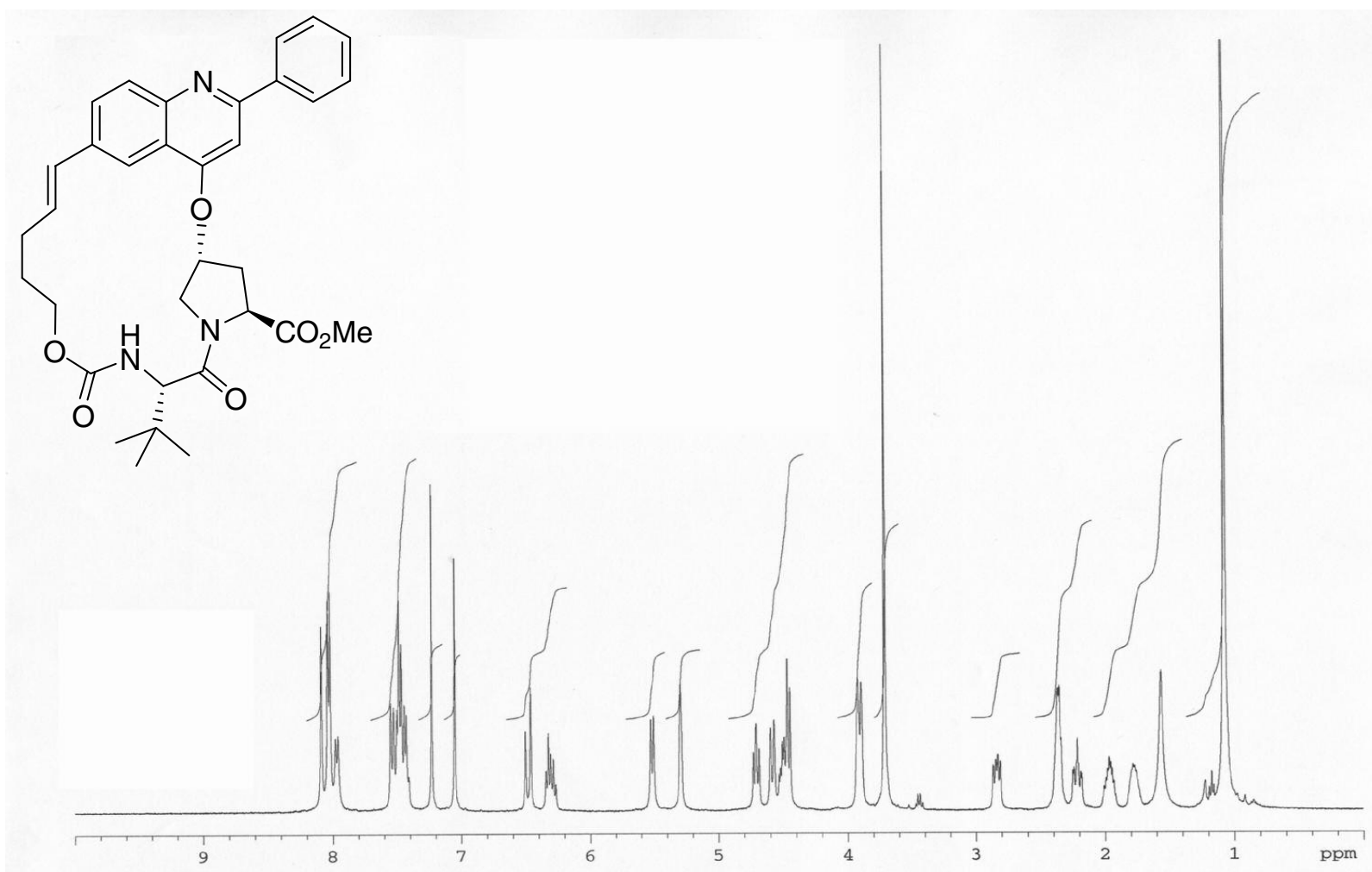

Method A HPLC Data

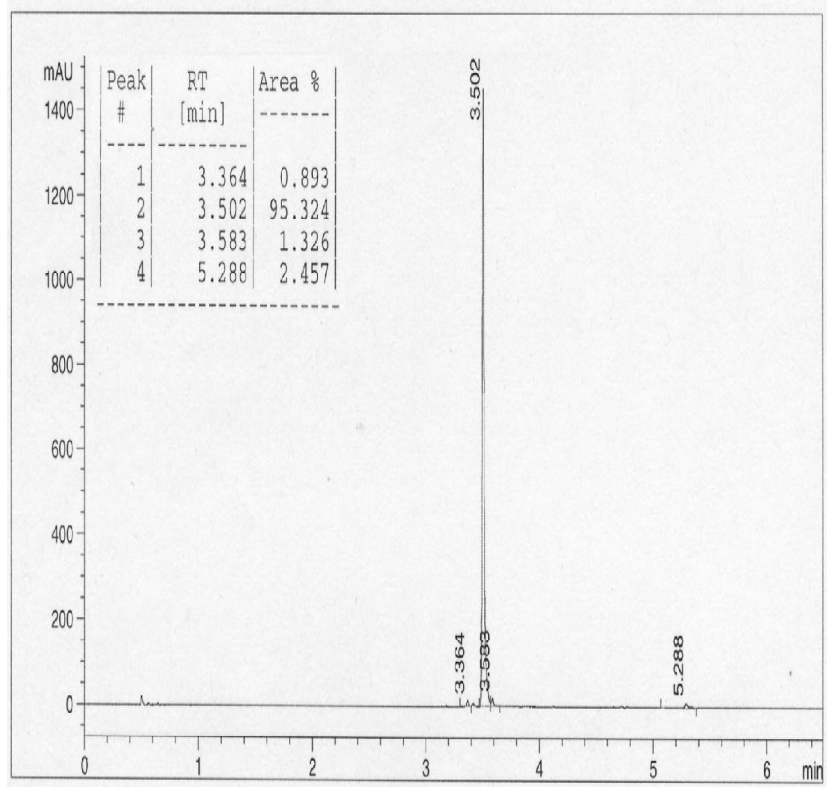

Method B HPLC Data

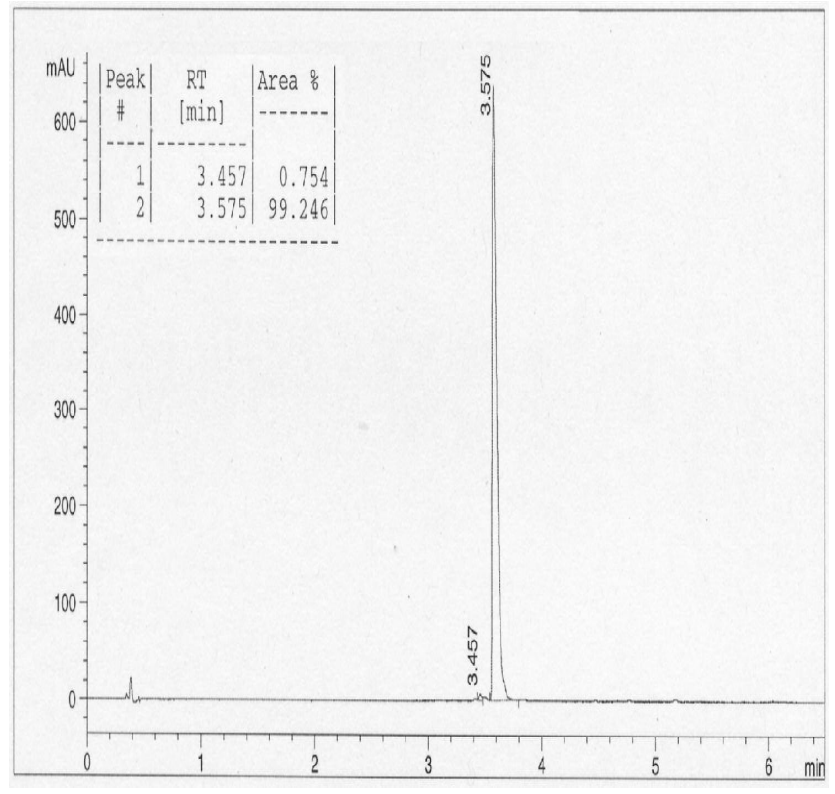




\section{NMR and HPLC Data for Compound 16}

${ }^{1} \mathrm{H}$ NMR Data $\left(400 \mathrm{MHz}, \mathrm{CD}_{3} \mathrm{OD}\right)$

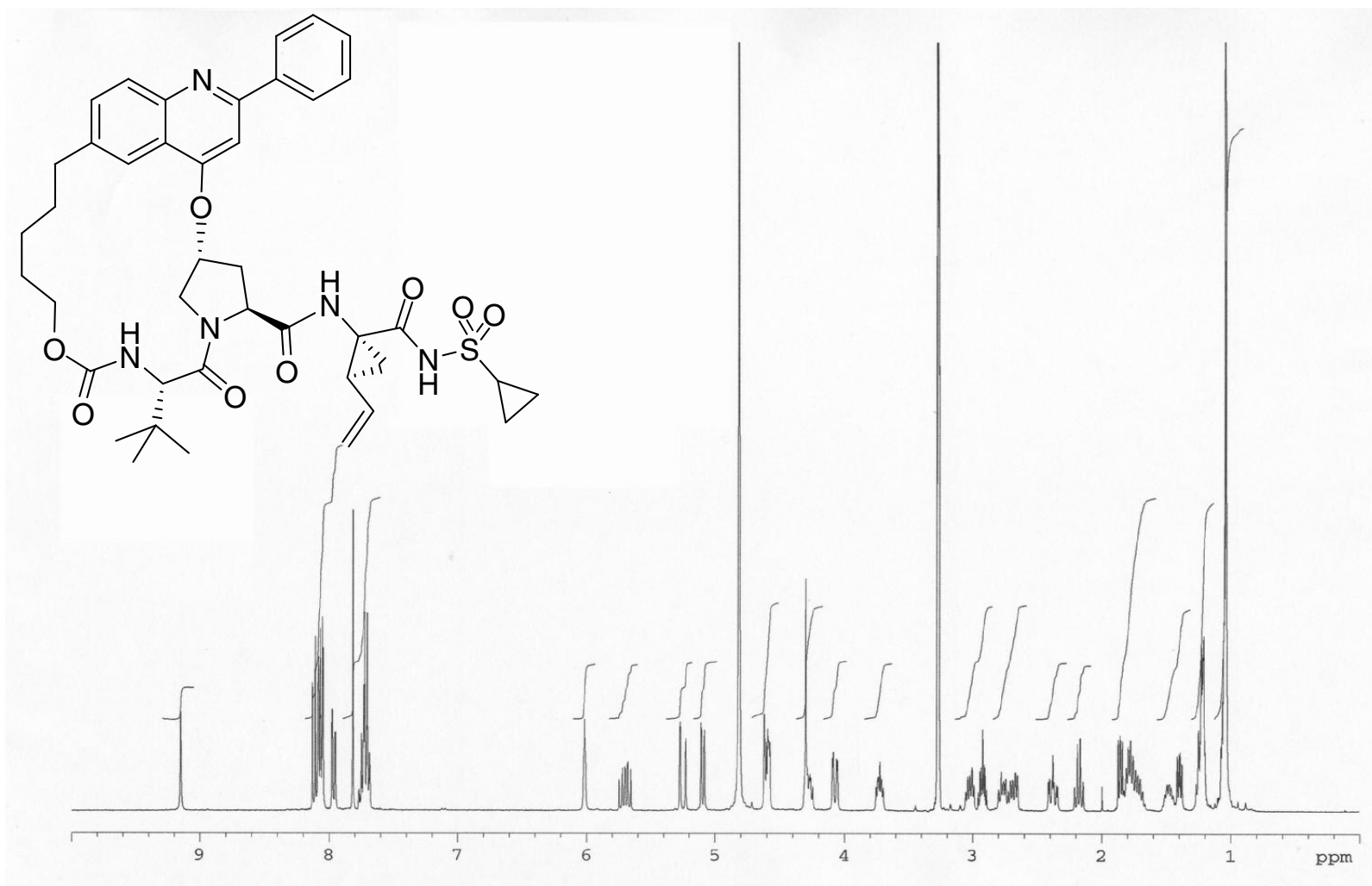

Method A HPLC Data

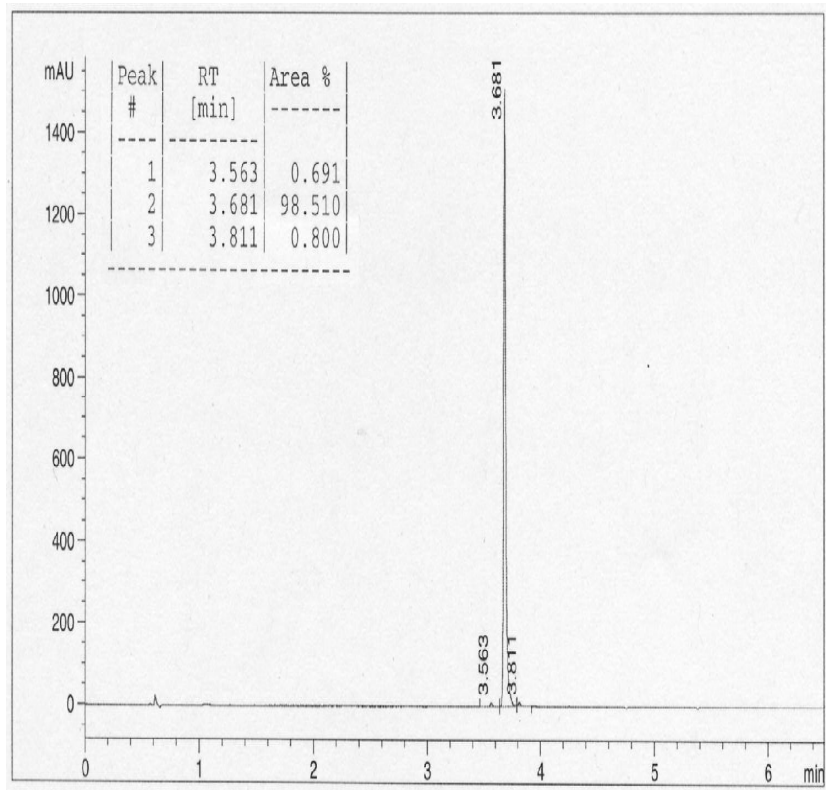

Method B HPLC Data

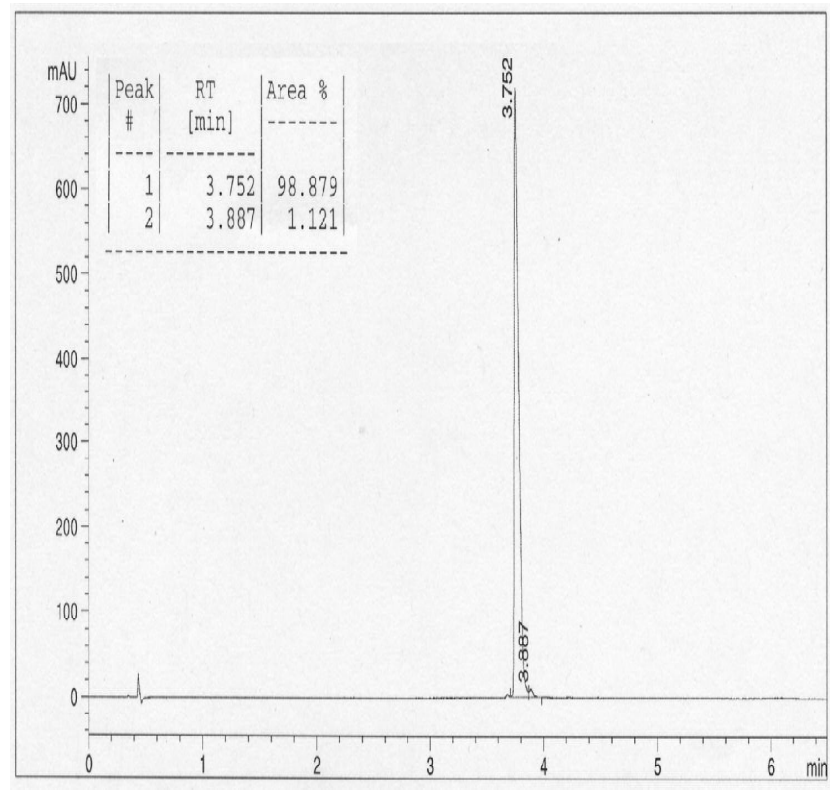


NMR and HPLC Data for Compound 19

${ }^{1} \mathrm{H}$ NMR Data $\left(400 \mathrm{MHz}, \mathrm{CD}_{3} \mathrm{OD}\right)$

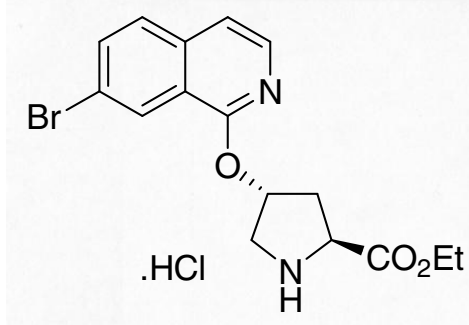

Method A HPLC Data

Method B HPLC Data 
NMR and HPLC Data for Compound 20

${ }^{1} \mathrm{H}$ NMR Data $\left(400 \mathrm{MHz}, \mathrm{CD}_{3} \mathrm{OD}\right)$
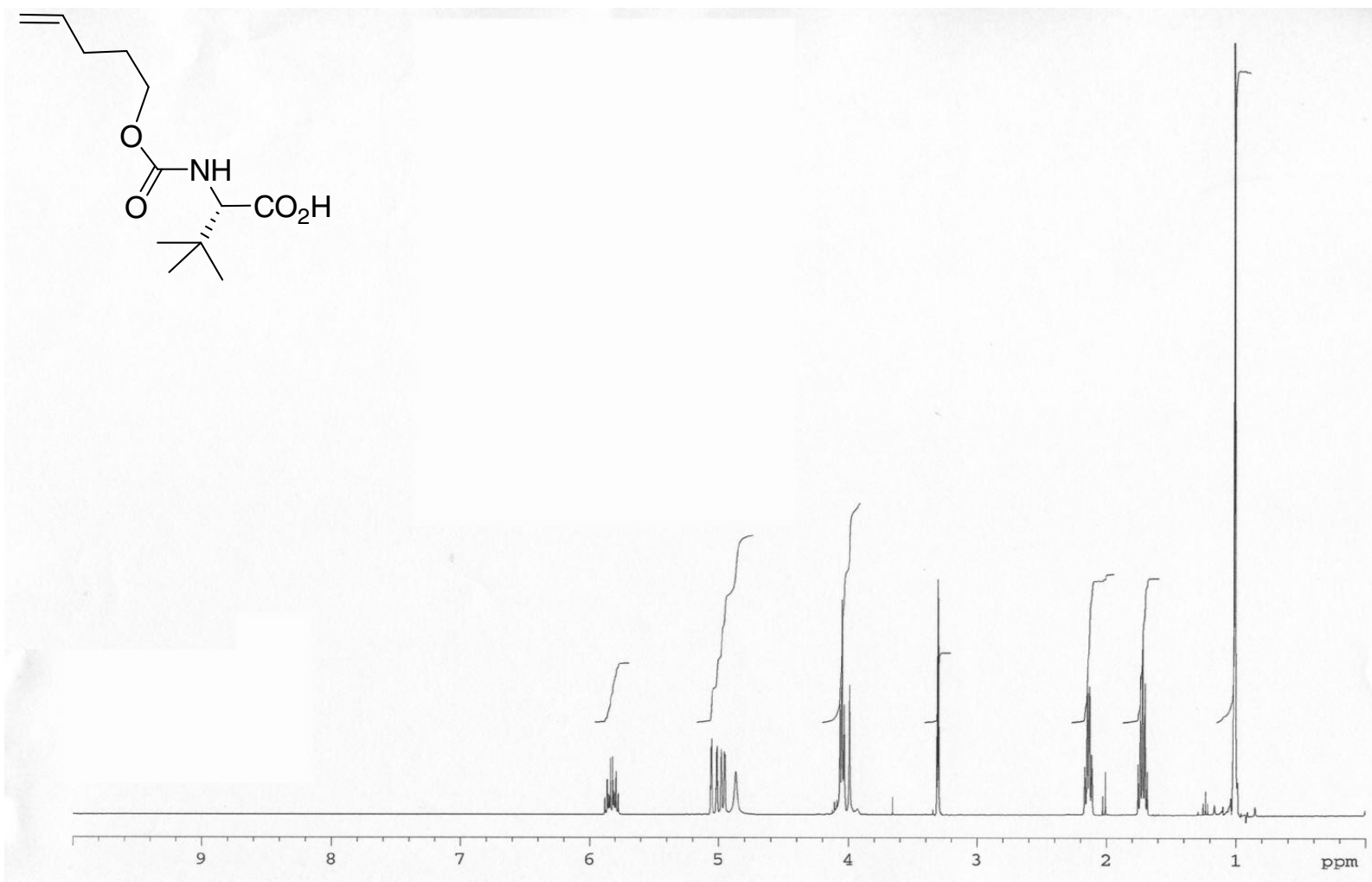

Method A HPLC Data

Method B HPLC Data 


\section{NMR and HPLC Data for Compound 21}

${ }^{1} \mathrm{H}$ NMR Data $\left(400 \mathrm{MHz}, \mathrm{CD}_{3} \mathrm{OD}\right)$<smiles>C=CCCCOC(=O)N[C@H](C(=O)N1C[C@H](Oc2nccc3ccc(Br)cc23)CC1C(=O)OCC)C(C)(C)C</smiles>

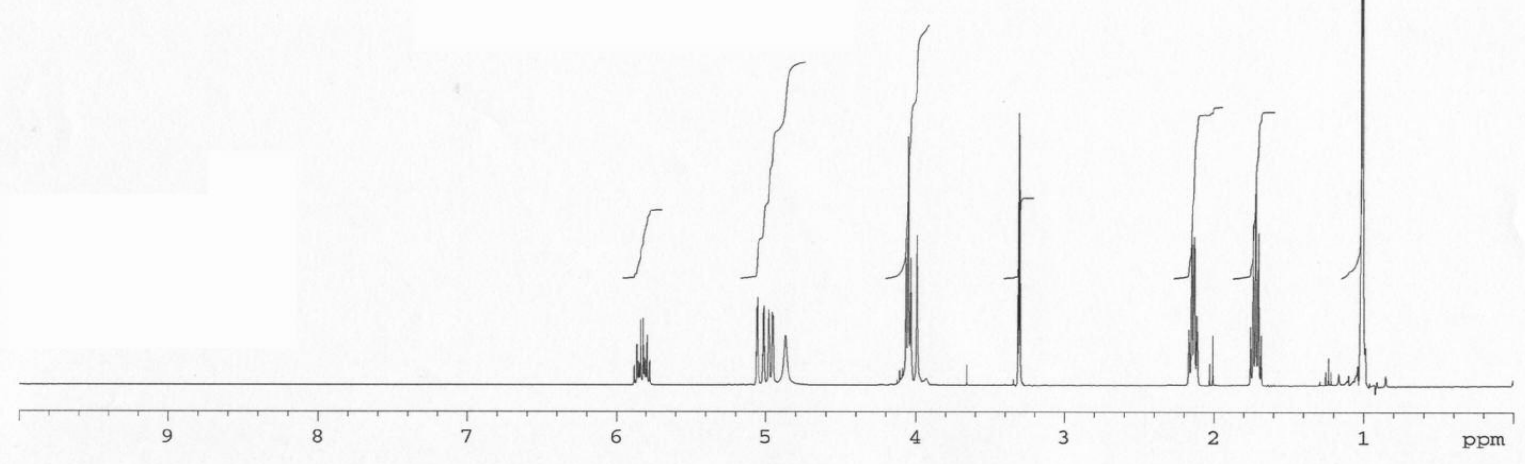

Method A HPLC Data

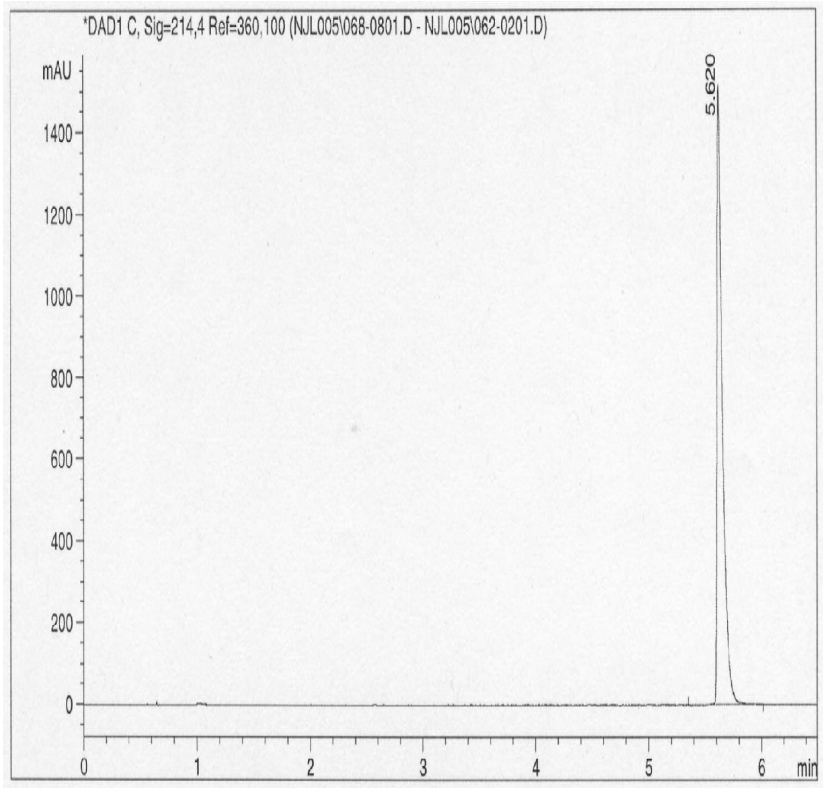

Method B HPLC Data

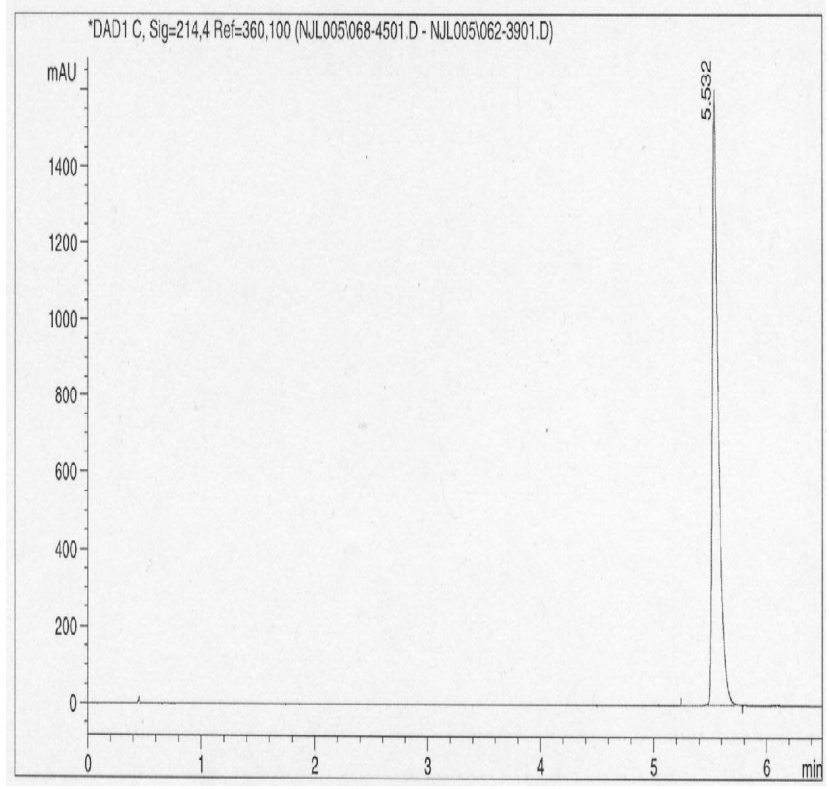


NMR and HPLC Data for Compound 22

${ }^{1} \mathrm{H}$ NMR Data (400 MHz, $\left.\mathrm{CD}_{3} \mathrm{OD}\right)$<smiles>C=CCCCOC(=O)N[C@@H](C(=O)OCC)C(=O)N1C[C@H](Oc2nccc3ccc(C=C)cc23)C[C@H]1C(C)(C)C</smiles>

Method A HPLC Data

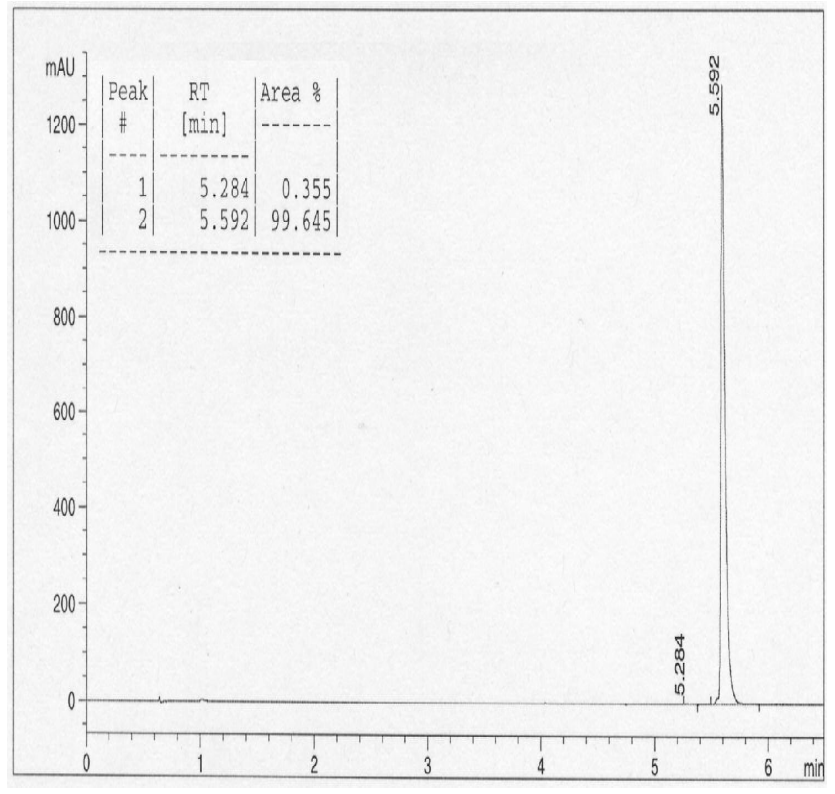

Method B HPLC Data

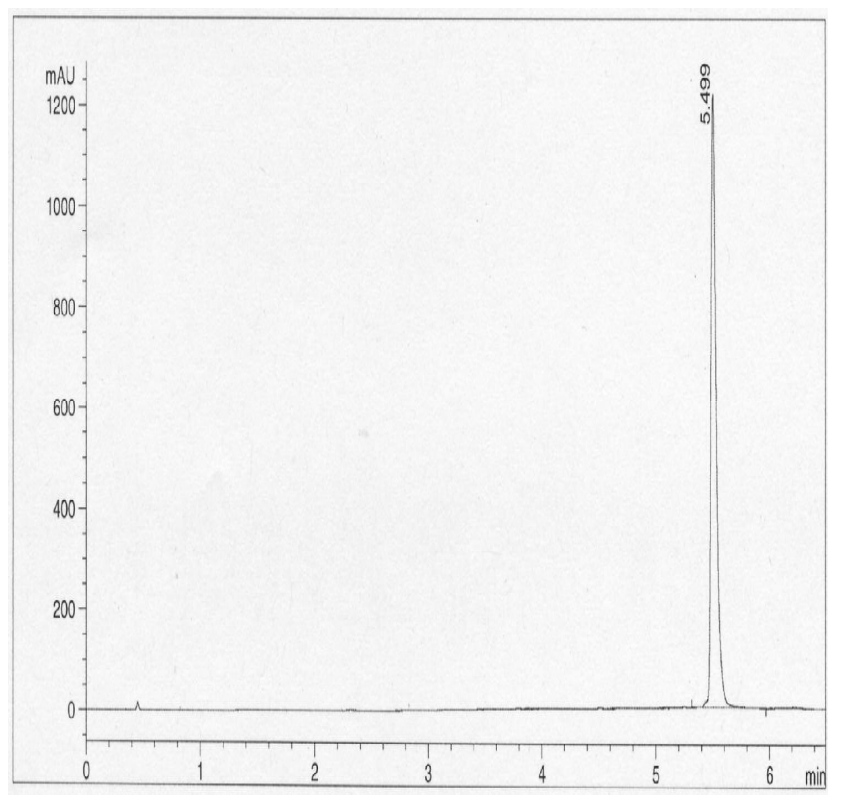


NMR and HPLC Data for Compound 23a

${ }^{1} \mathrm{H}$ NMR Data $\left(400 \mathrm{MHz}, \mathrm{CD}_{3} \mathrm{OD}\right)$

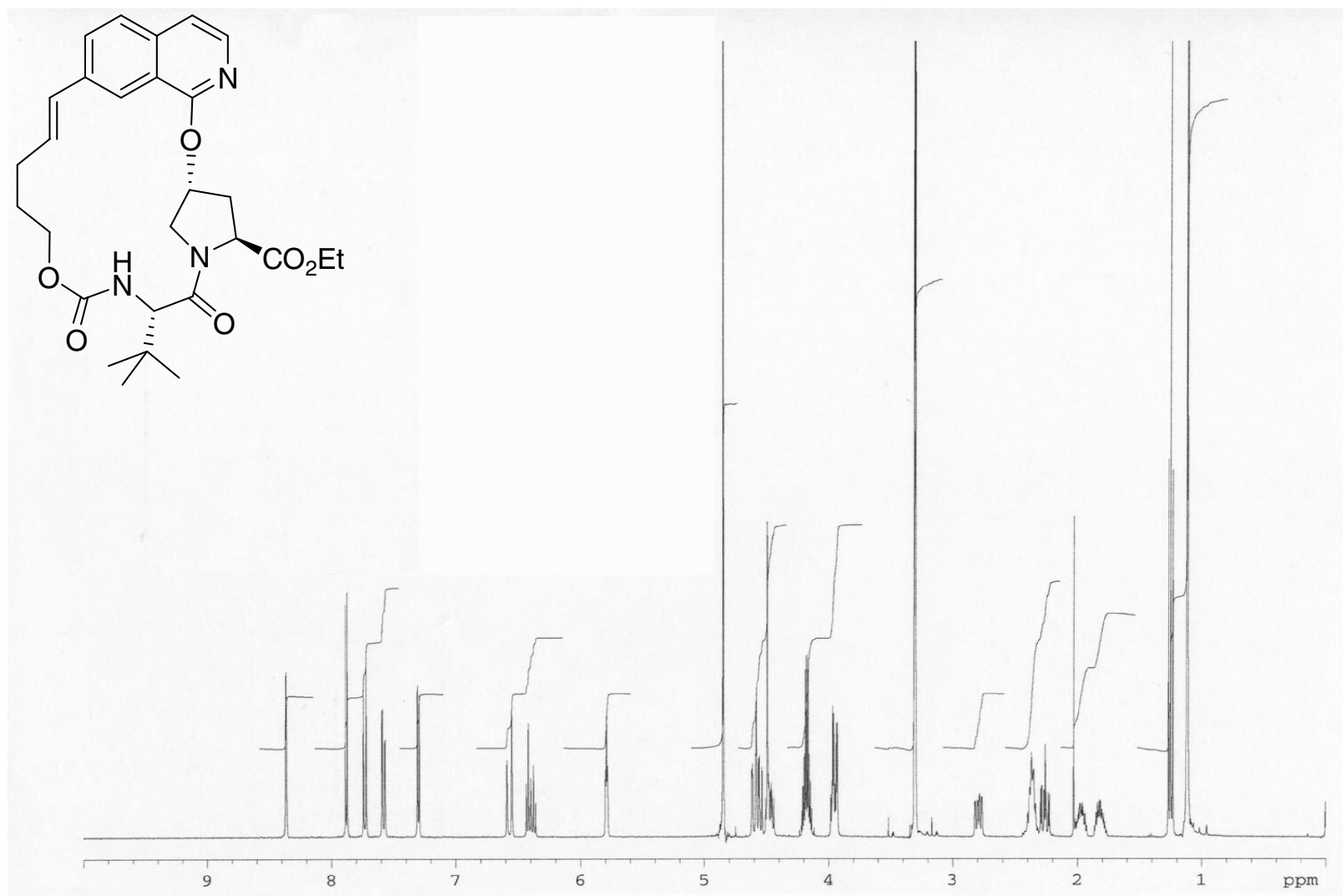

Method A HPLC Data

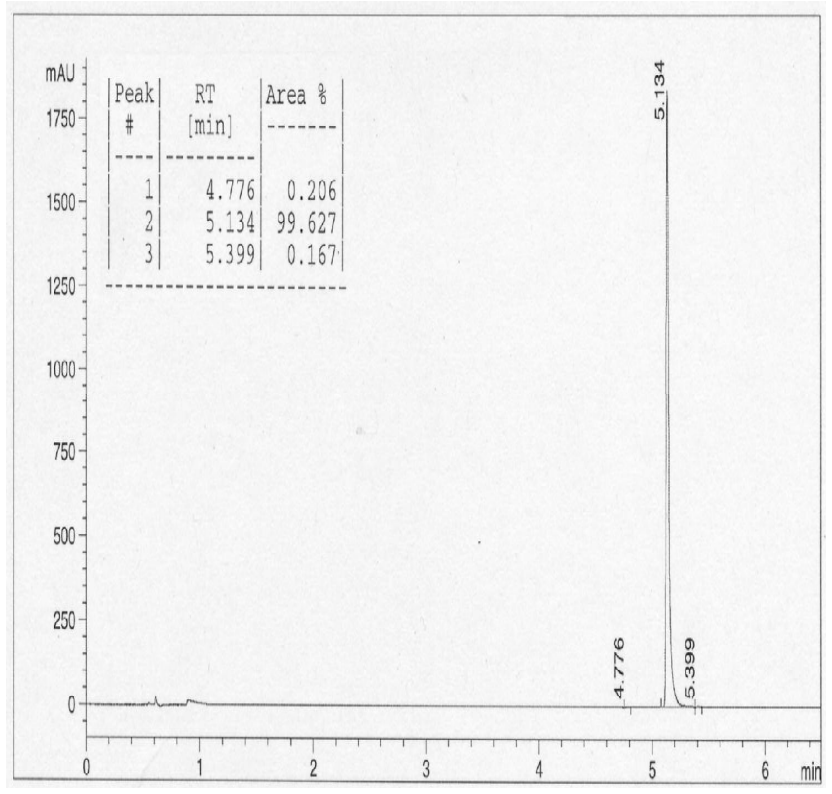

Method B HPLC Data

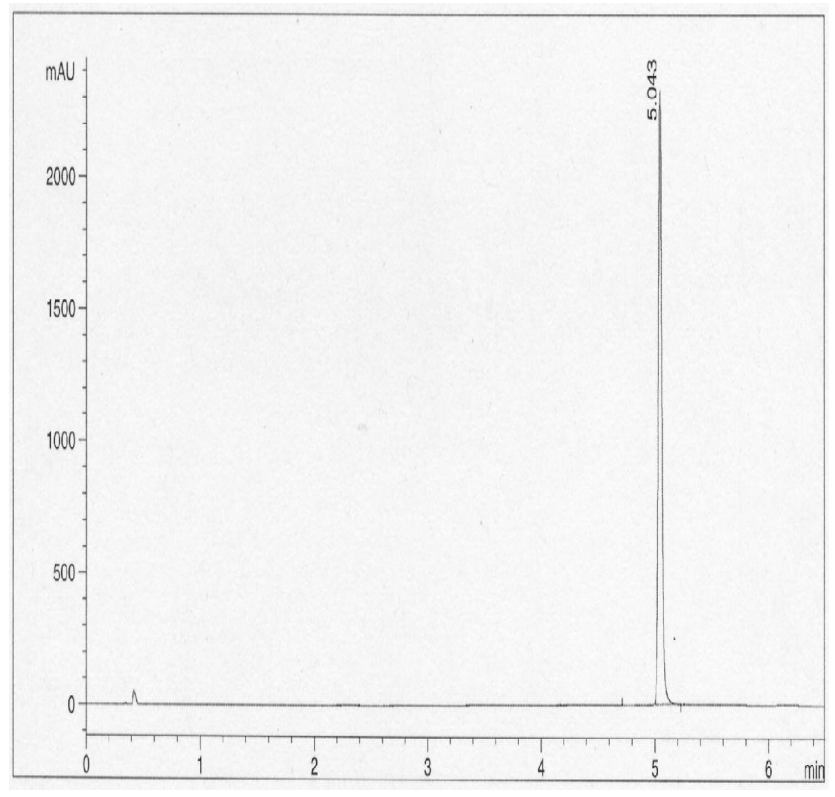


NMR and HPLC Data for Compound 23b

${ }^{1} \mathrm{H}$ NMR Data $\left(400 \mathrm{MHz}, \mathrm{CD}_{3} \mathrm{OD}\right)$<smiles></smiles>

Method A HPLC Data

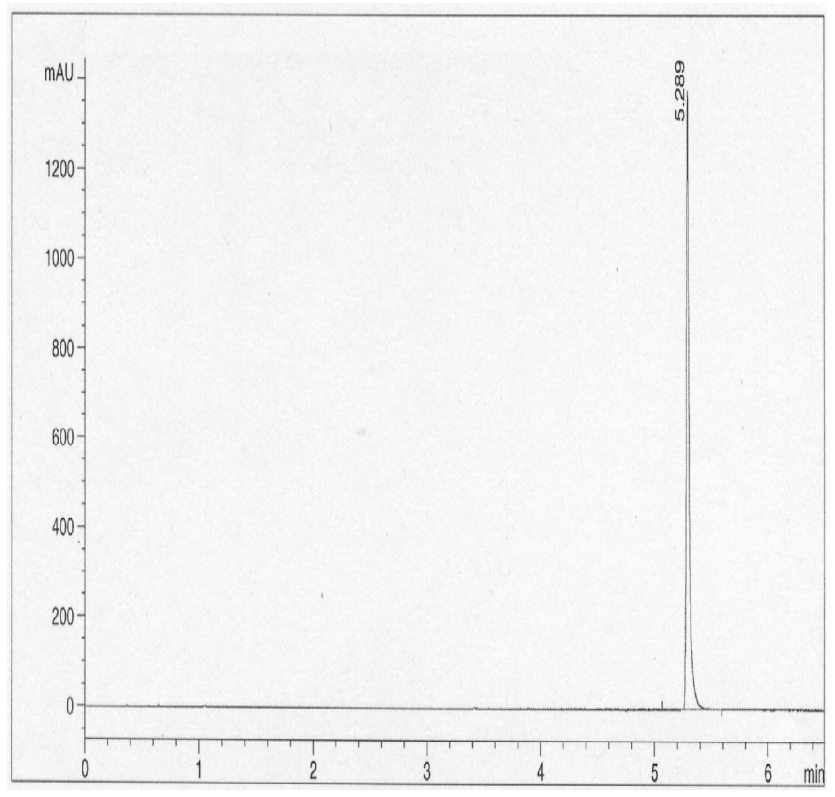

Method B HPLC Data

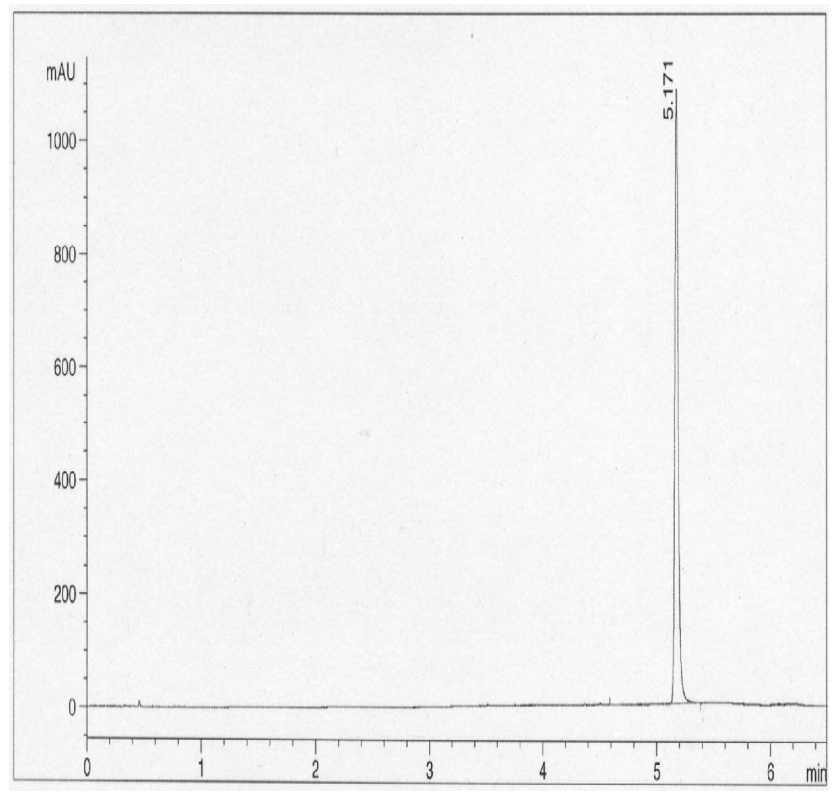


NMR and HPLC Data for Compound 25a

${ }^{1} \mathrm{H}$ NMR Data $\left(400 \mathrm{MHz}, \mathrm{CDCl}_{3}\right.$ )

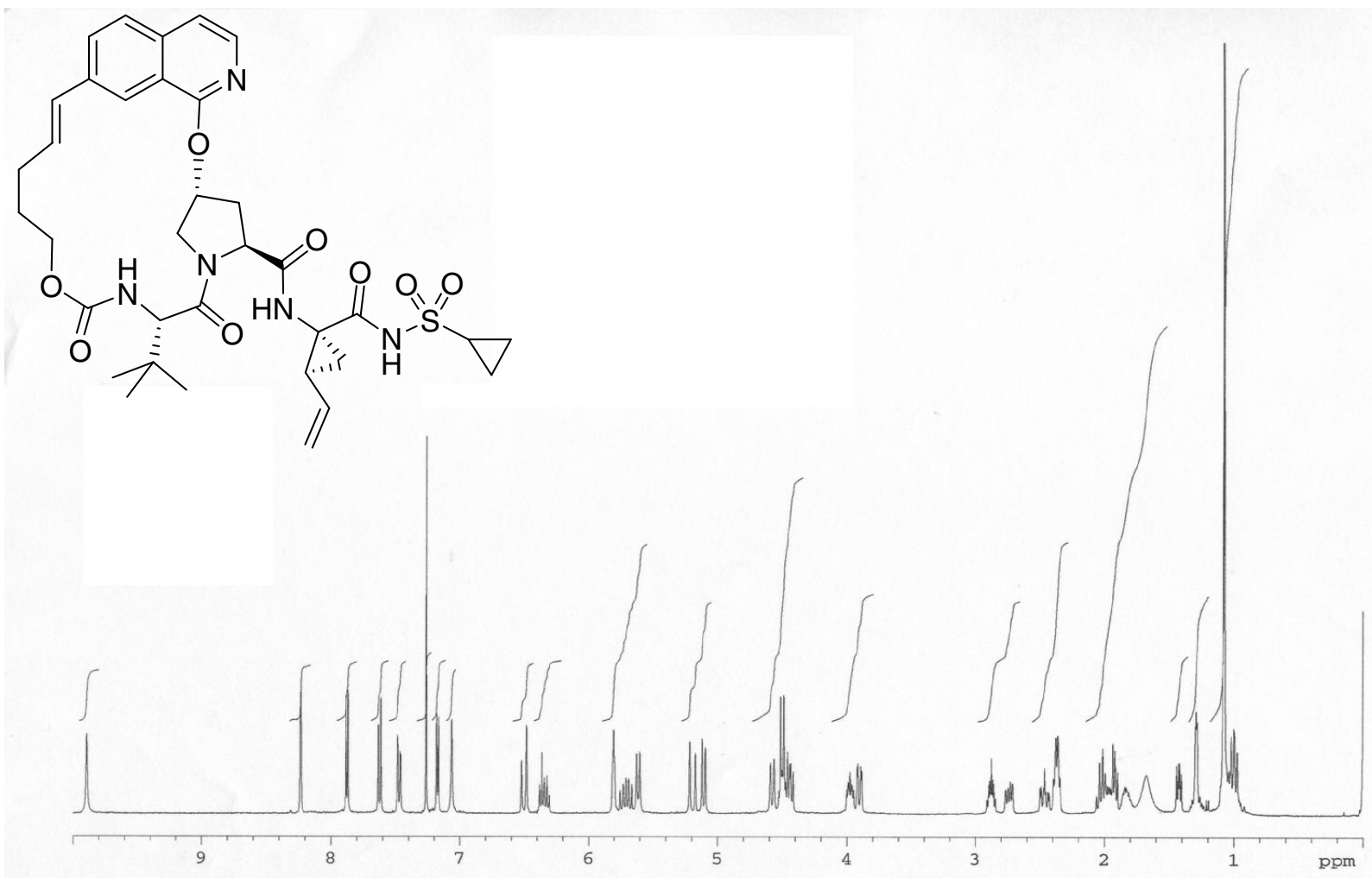

Method A HPLC Data

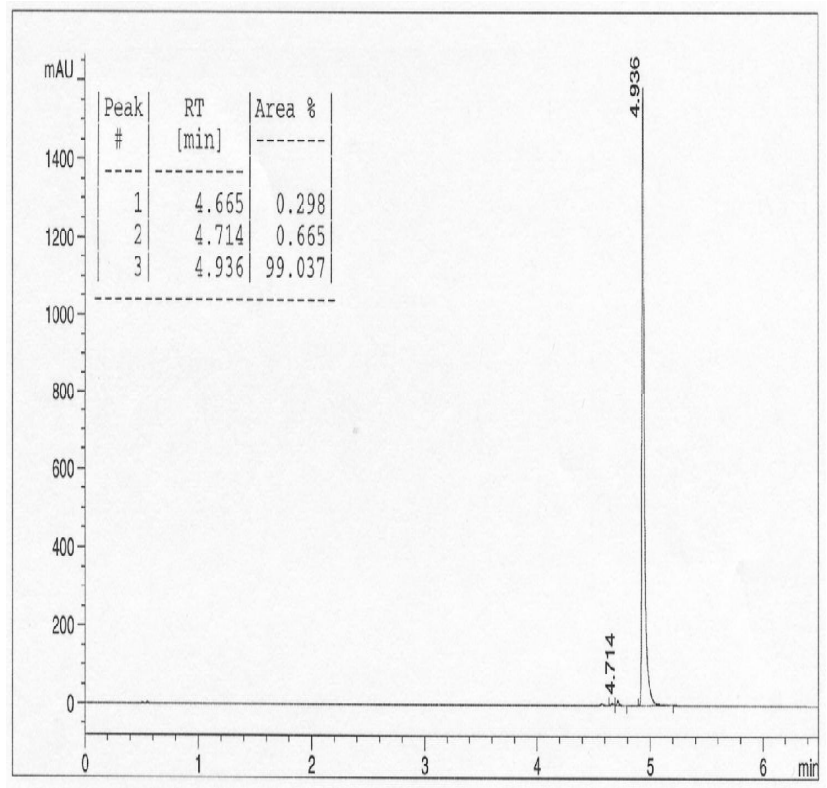

Method B HPLC Data

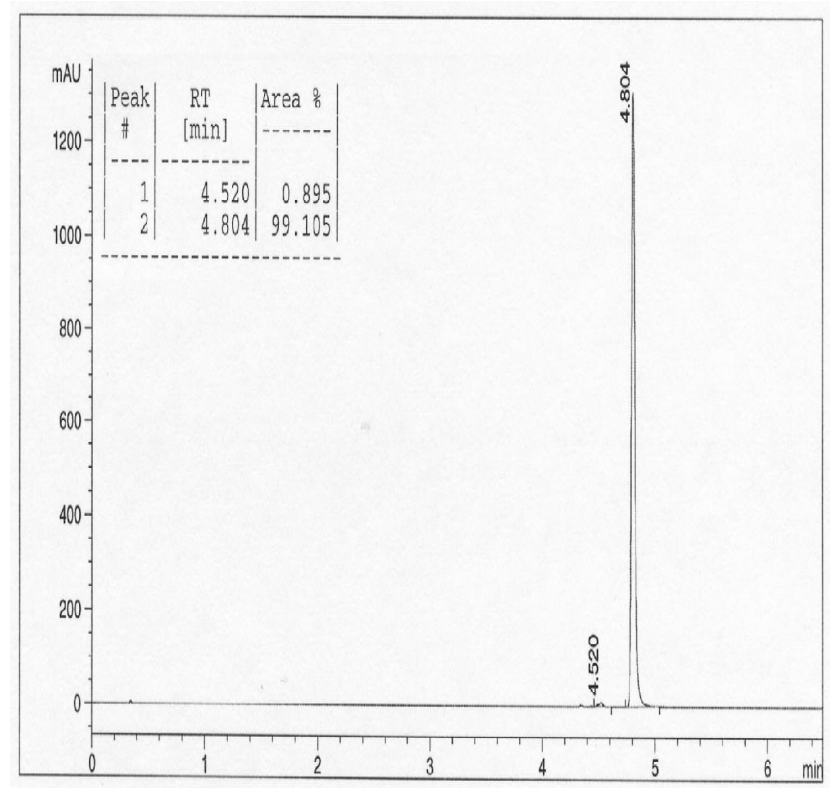


NMR and HPLC Data for Compound 25b

${ }^{1} \mathrm{H}$ NMR Data (400 MHz, d $\mathrm{d}^{6}$-DMSO)

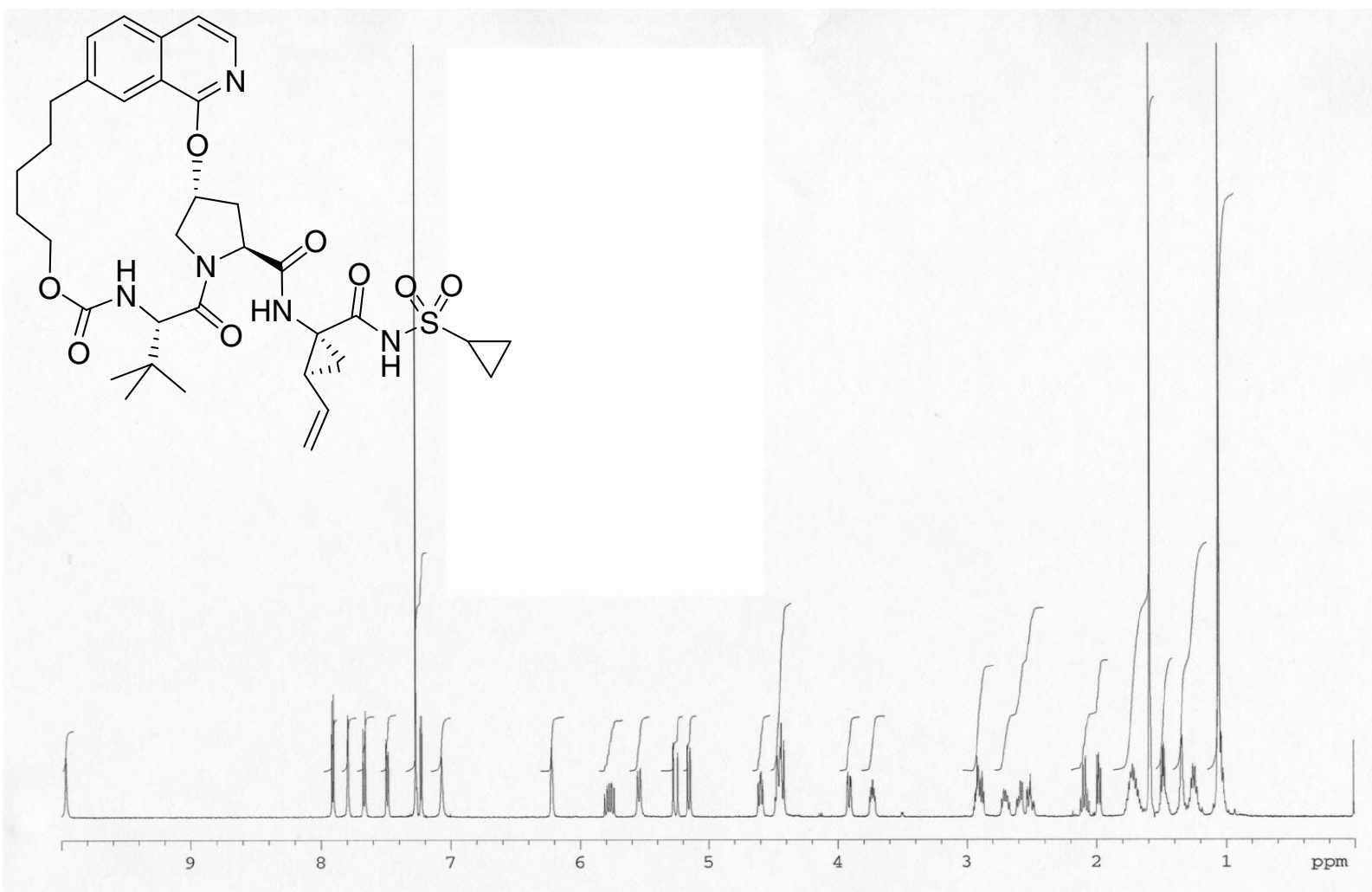

Method A HPLC Data

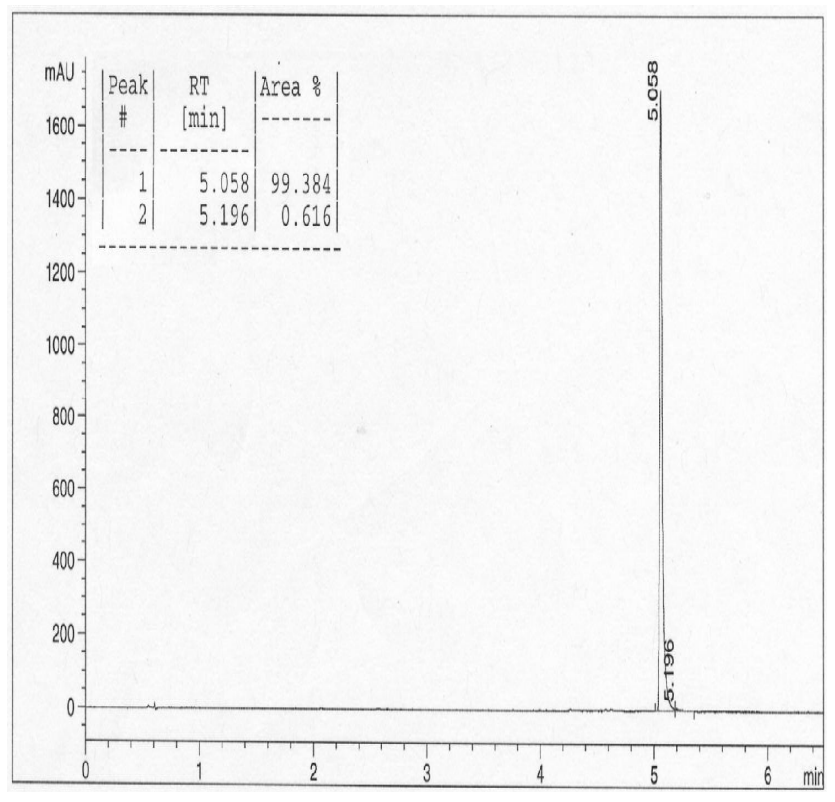

Method B HPLC Data

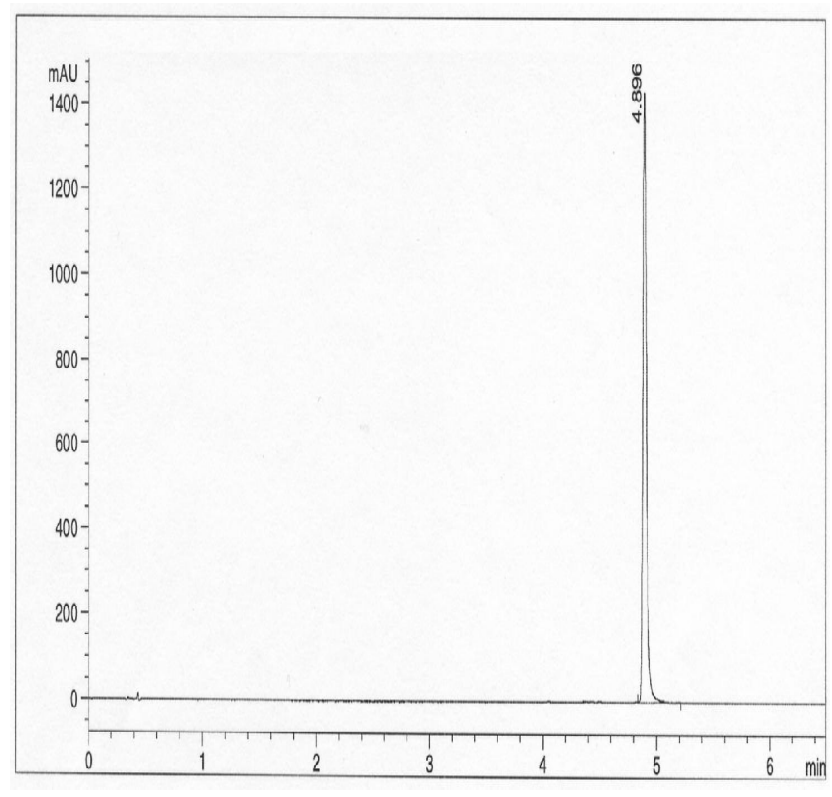


Characterization data

\begin{tabular}{|c|c|c|c|c|c|c|c|c|c|c|}
\hline Compound & L\# & NB Page & \# & NMR & HRMS & $\begin{array}{l}\mathrm{LC} \\
\mathrm{A}\end{array}$ & $\begin{array}{l}\mathrm{LC} \\
\mathrm{B}\end{array}$ & $\begin{array}{l}\text { Sample } \\
\text { Avail. }\end{array}$ & $\mathrm{CHN}$ & $\alpha \mathrm{D}$ \\
\hline & & $\begin{array}{c}0247275- \\
0090 \\
0247275- \\
0091\end{array}$ & 6 & $\mathrm{x}$ & $\mathrm{x}$ & $\mathrm{x}$ & $\mathrm{x}$ & & & \\
\hline & & $\begin{array}{c}0247275- \\
0092 \\
0247275- \\
0093\end{array}$ & 7 & $\mathrm{x}$ & $\mathrm{x}$ & $\mathrm{X}$ & $\mathrm{X}$ & & & \\
\hline & & $\begin{array}{c}219114- \\
24 \mathrm{~A}\end{array}$ & $8 b$ & $\mathrm{x}$ & & $\mathrm{x}$ & $\mathrm{X}$ & & & \\
\hline & 002110011 & $\begin{array}{c}0268408- \\
0035\end{array}$ & $8 c$ & $\mathrm{x}$ & $\mathrm{x}$ & & & & & \\
\hline & 002038946 & $\begin{array}{c}219803-31- \\
1\end{array}$ & $8 d$ & $\mathrm{X}$ & $\mathrm{x}$ & $\mathrm{X}$ & $\mathrm{X}$ & & & \\
\hline & & $\begin{array}{c}0247275- \\
0095\end{array}$ & $9 \mathbf{a}$ & $\mathrm{x}$ & $\mathrm{x}$ & $\mathrm{X}$ & $\mathrm{X}$ & & & \\
\hline & 002119866 & $\begin{array}{c}0267808- \\
0087\end{array}$ & $9 \mathrm{~b}$ & $\mathrm{X}$ & & $\mathrm{X}$ & $\mathrm{X}$ & & & \\
\hline & 002119853 & $\begin{array}{c}0267808- \\
0068\end{array}$ & $9 c$ & $\mathrm{X}$ & $\mathrm{x}$ & ND & ND & & & \\
\hline & 002119868 & $\begin{array}{c}0267808- \\
0086\end{array}$ & 9d & $\mathrm{x}$ & & $\mathrm{X}$ & $\mathrm{x}$ & & & \\
\hline
\end{tabular}




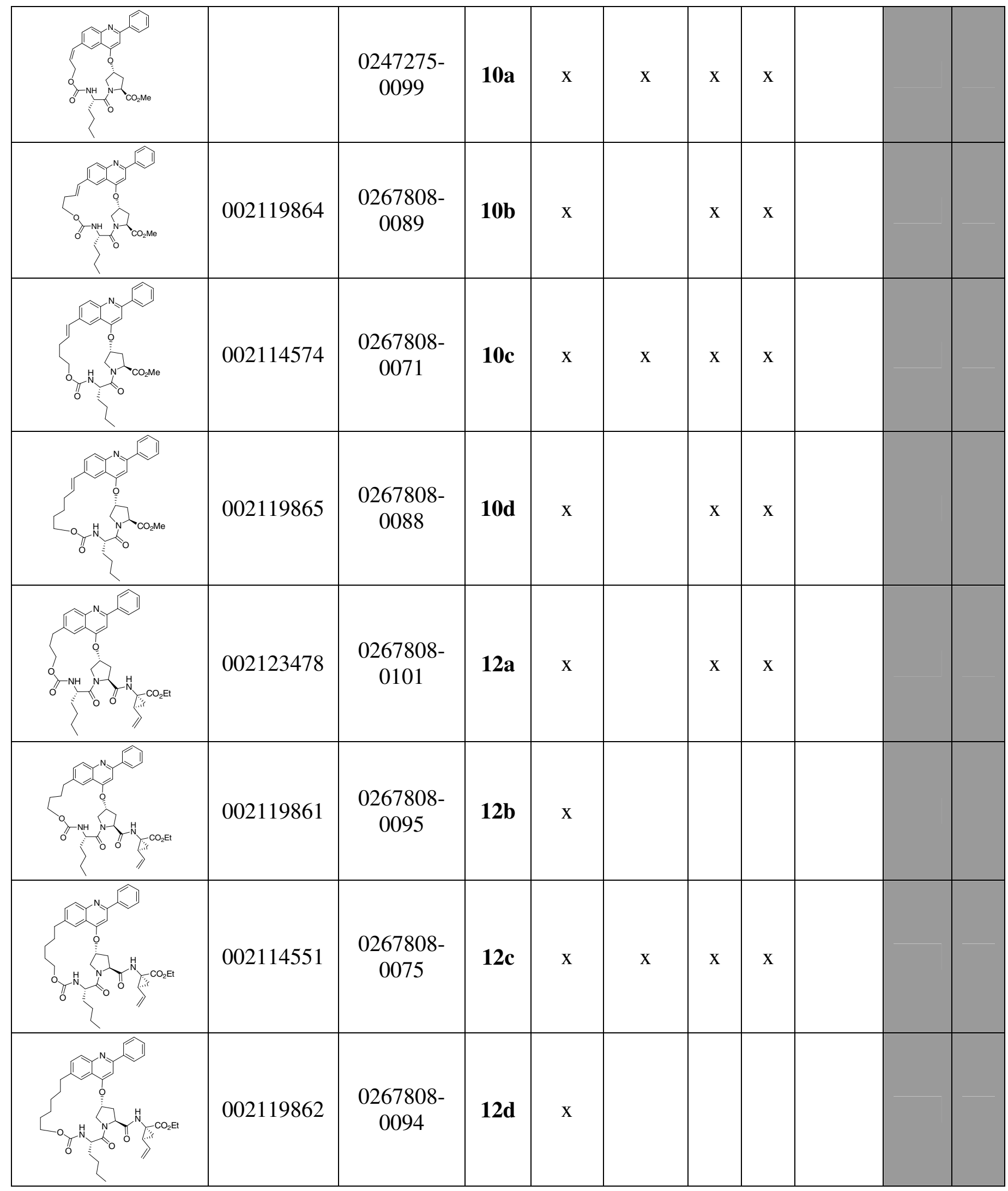




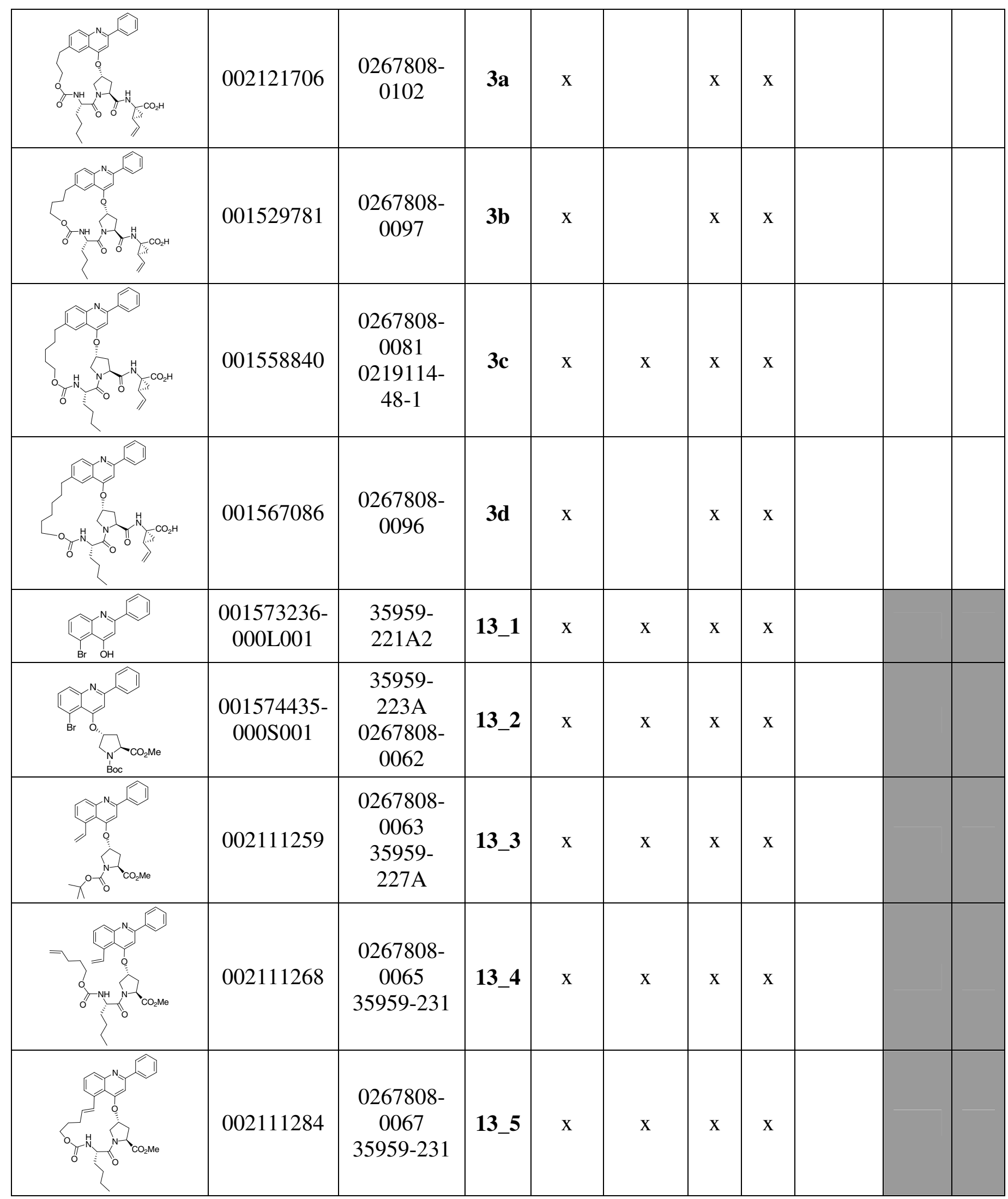




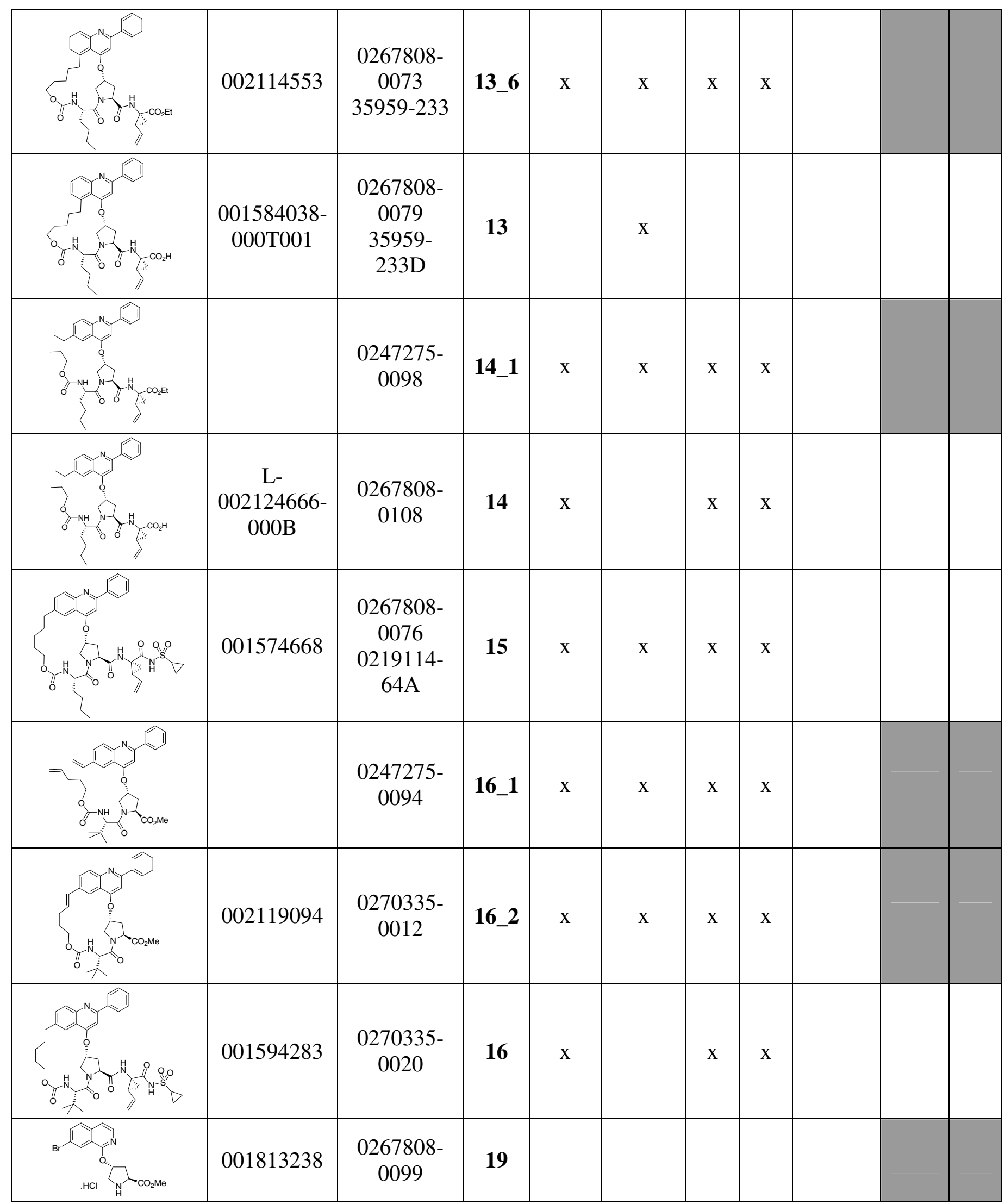




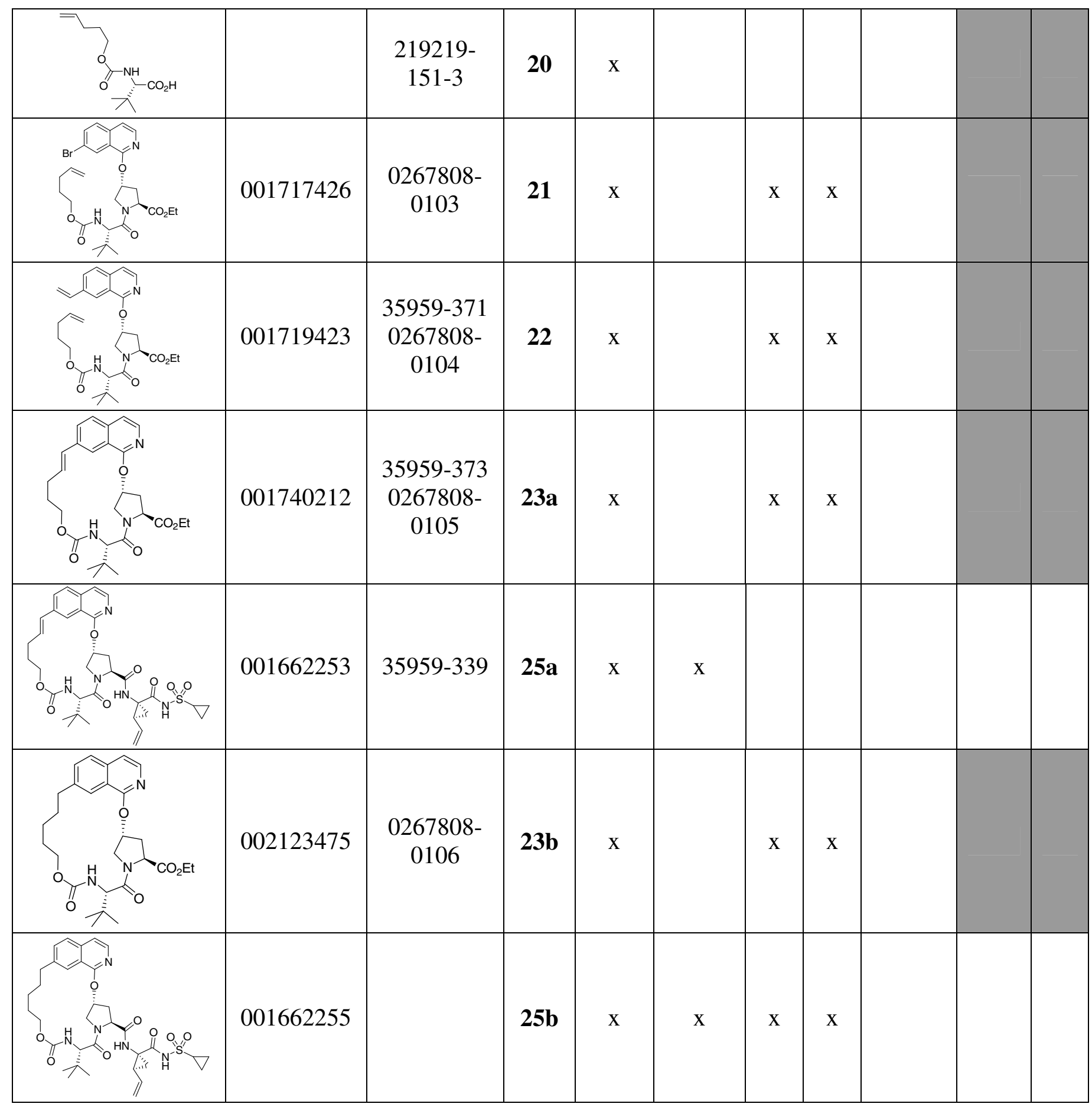


